\title{
Sulfur Oxidation Increases the Rate of HIRE-type [1.4]Thiazepinone Ring Expansion and Influences the Conformation of a Medium-Sized Heterocyclic Scaffold
}

\author{
Elena Reutskaya, ${ }^{a}$ Alexander Sapegin, ${ }^{a}$ Stefan Peintner, ${ }^{b}$ Máté Erdélyi ${ }^{b}$ and Mikhail \\ Krasavin $^{a, *}$ \\ ${ }^{a}$ Institute of Chemistry, Saint Petersburg State University, Saint Petersburg 199034 Russia \\ ${ }^{b}$ Department of Chemistry - BMC, Uppsala University, SE-751 23 Uppsala, Sweden \\ * Corresponding Author; phone: + 7931 3617872, fax: +7 8124286939 . \\ E-mail: $\underline{\text { m.krasavin@spbu.ru }}$
}

\section{Table of Contents}

Crystallographic data for compounds 12a, 12e, 12n, 13a, 13b, 13e, 13f, 13h,

13l, 13n, 14b and 14c

Example of kobs calculation (compound 9a)

S15-S19

Copies of ${ }^{1} \mathrm{H}$ and ${ }^{13} \mathrm{C}\left\{{ }^{1} \mathrm{H}\right\}$ NMR spectra of all new compounds

S20-S83

NMR assignment of 13a

EXSY/NOESY

S85-S86

Variable temperature NMR

S86-S88

NOE based distance calculations

S88-S89

Monte-Carlo conformational search

S89-S91

Relevant NMR spectra

S92-S95

Ensemble atom coordinates

S95-S102

References

S103 
Crystallographic data for compounds 12a, 12e, 12n, 13a, 13b, 13e, 13f, 13h, 13l, 13n, 14b and 14c

X-ray single crystal analyses were performed on Agilent Technologies «Xcalibur» and «Supernova» diffractometres with monochromated $\mathrm{Mo} \mathrm{K} \alpha$ or $\mathrm{Cu} \alpha$ radiation, respectively. In all cases, the temperature was kept at 100(2) K during data collection. The structures has been solved with the ShelXT ${ }^{1}$ structure solution programs using Intrinsic Phasing and refined with the ShelXT ${ }^{1}$ refinement incorporated in the OLEX2 program package ${ }^{2}$ using Least Square minimization. Empirical absorption correction was applied in CrysAlisPro ${ }^{3}$ program complex using spherical harmonics, implemented in SCALE3 ABSPACK scaling algorithm.

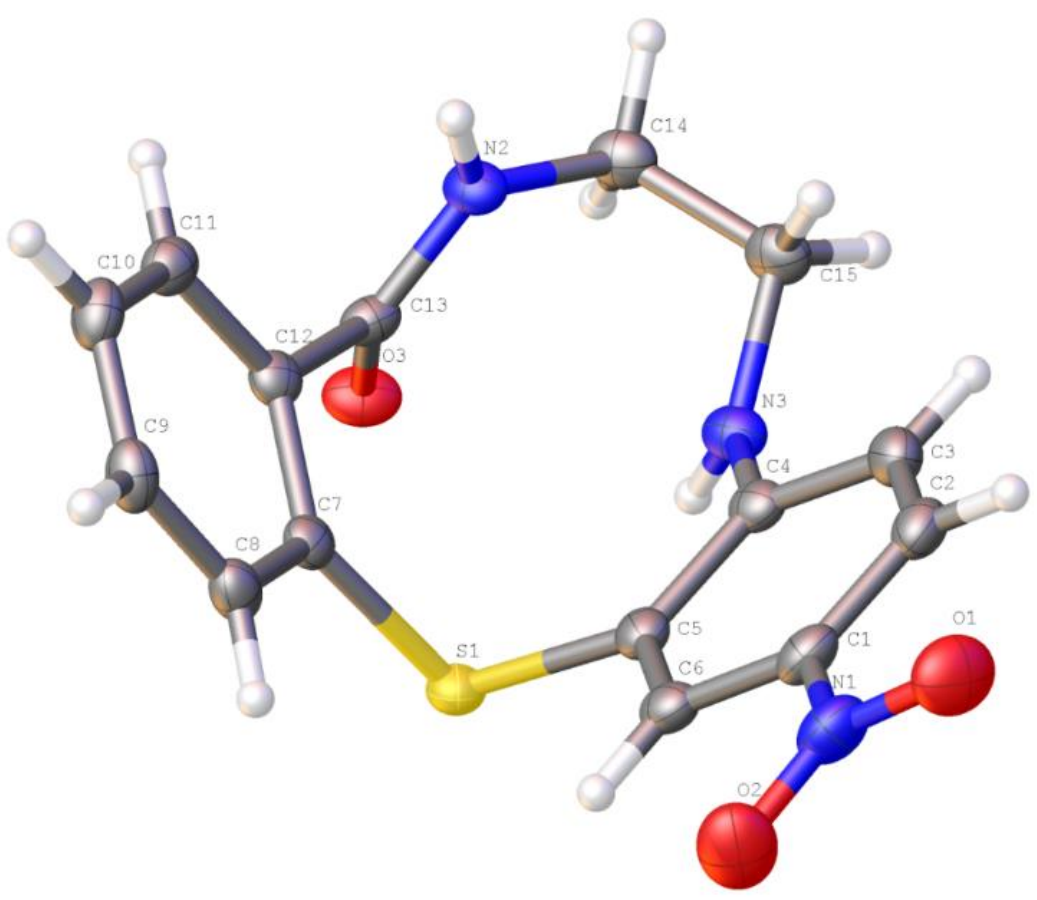

Figure S1. ORTEP representation of compound 12a displaying thermal ellipsoids at 50\% 


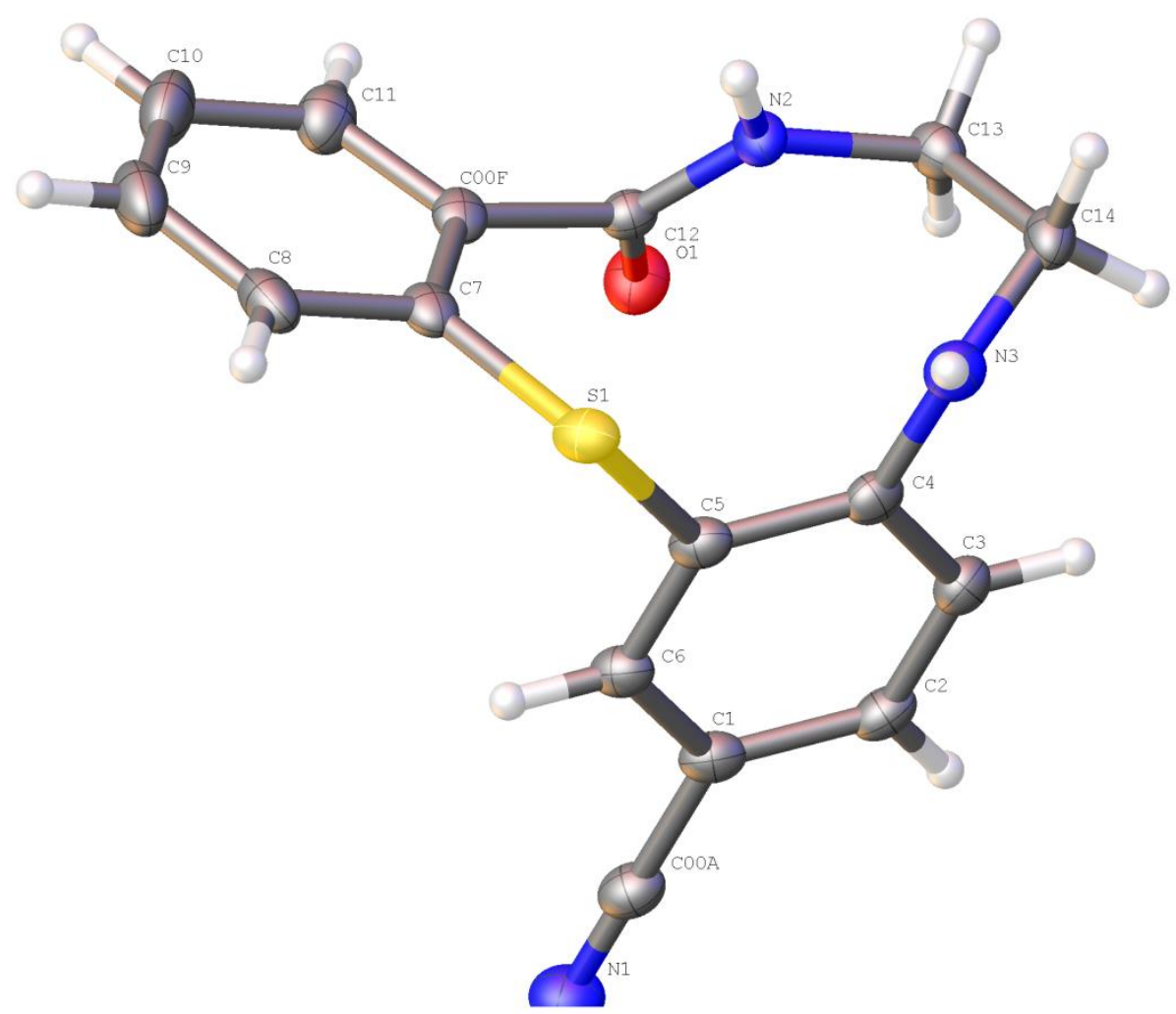

Figure S2. ORTEP representation of compound 12e displaying thermal ellipsoids at 50\%

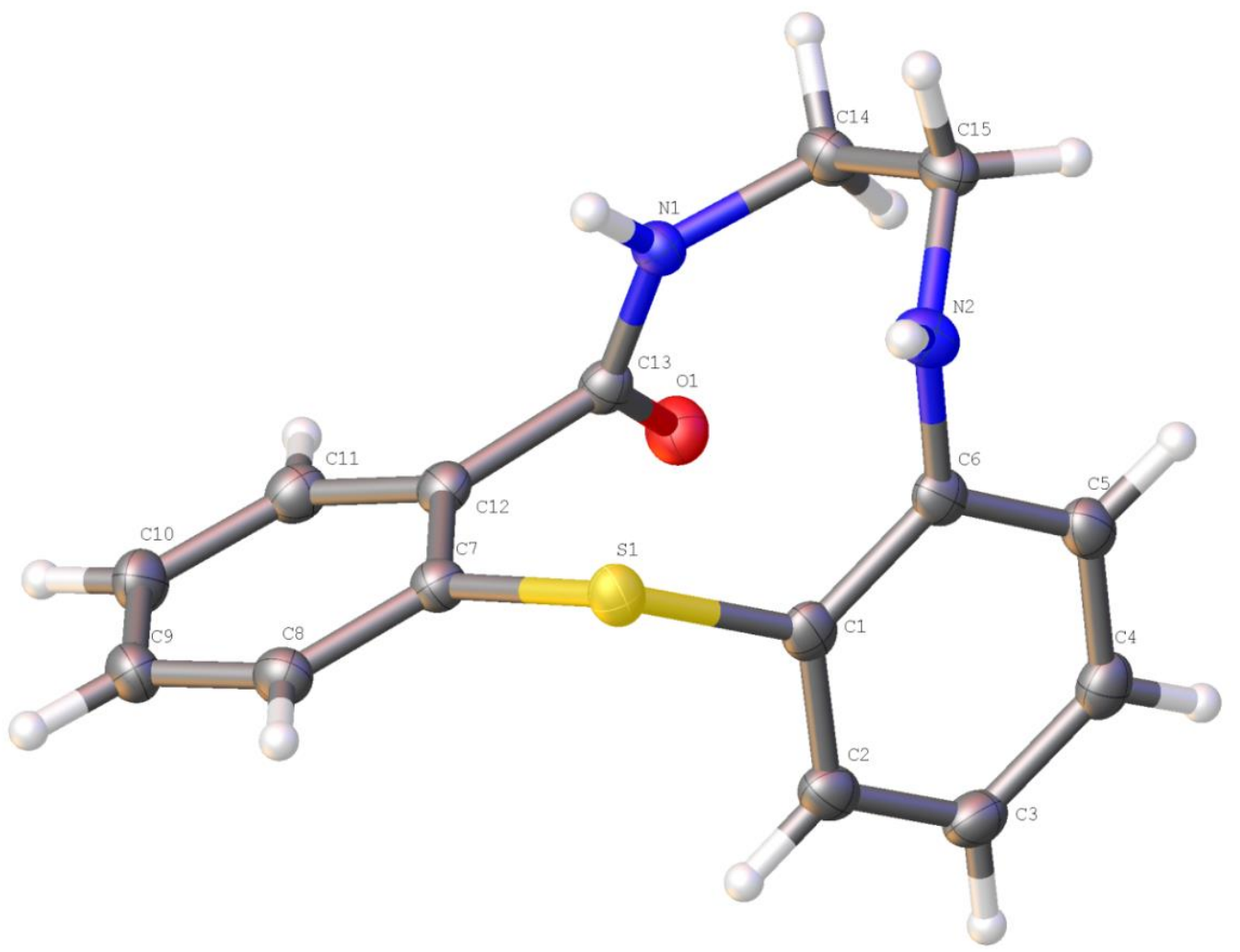

Figure S3. ORTEP representation of compound 12n displaying thermal ellipsoids at 50\% 


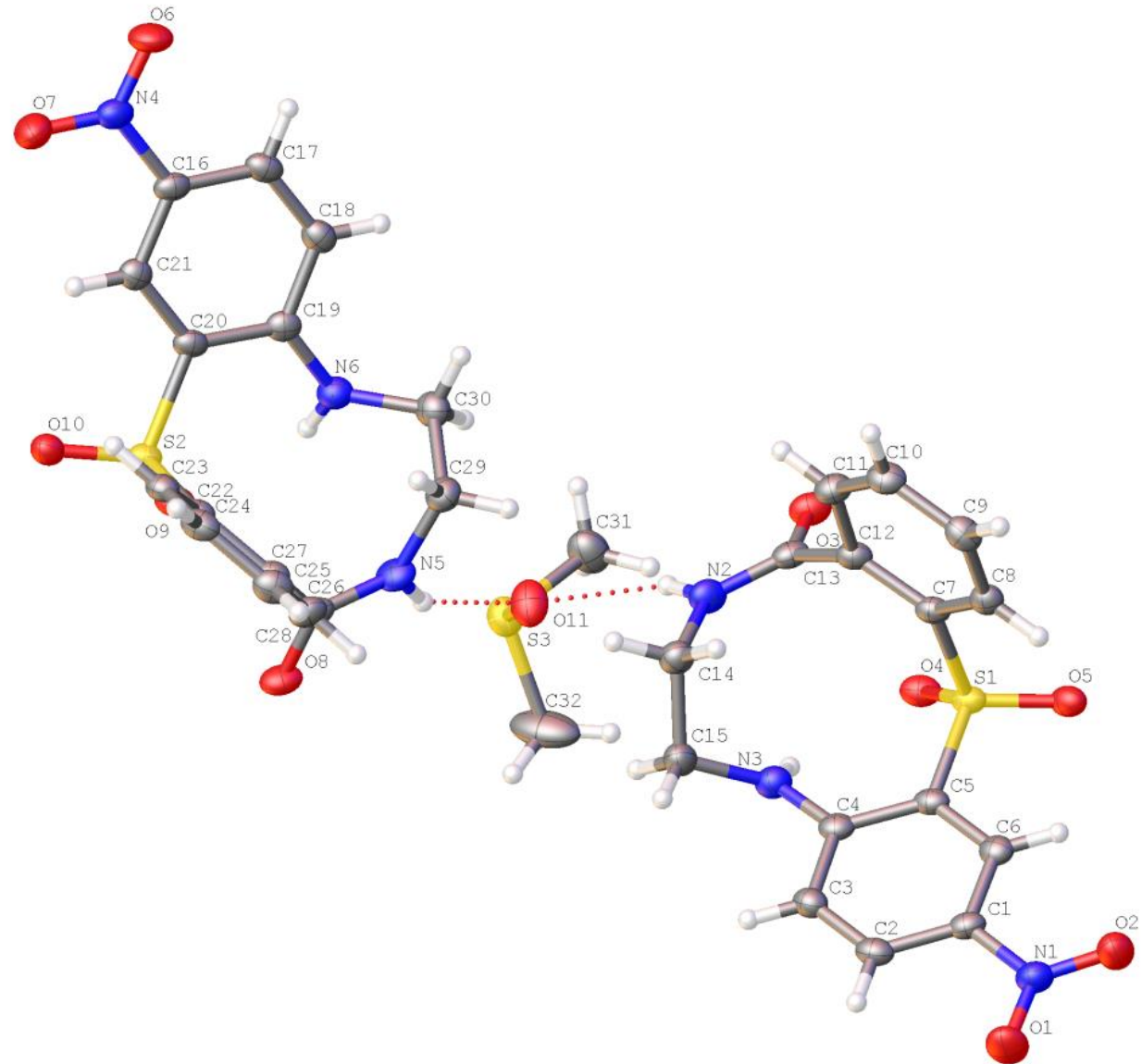

Figure S4. ORTEP representation of compound 13a displaying thermal ellipsoids at 50\% 


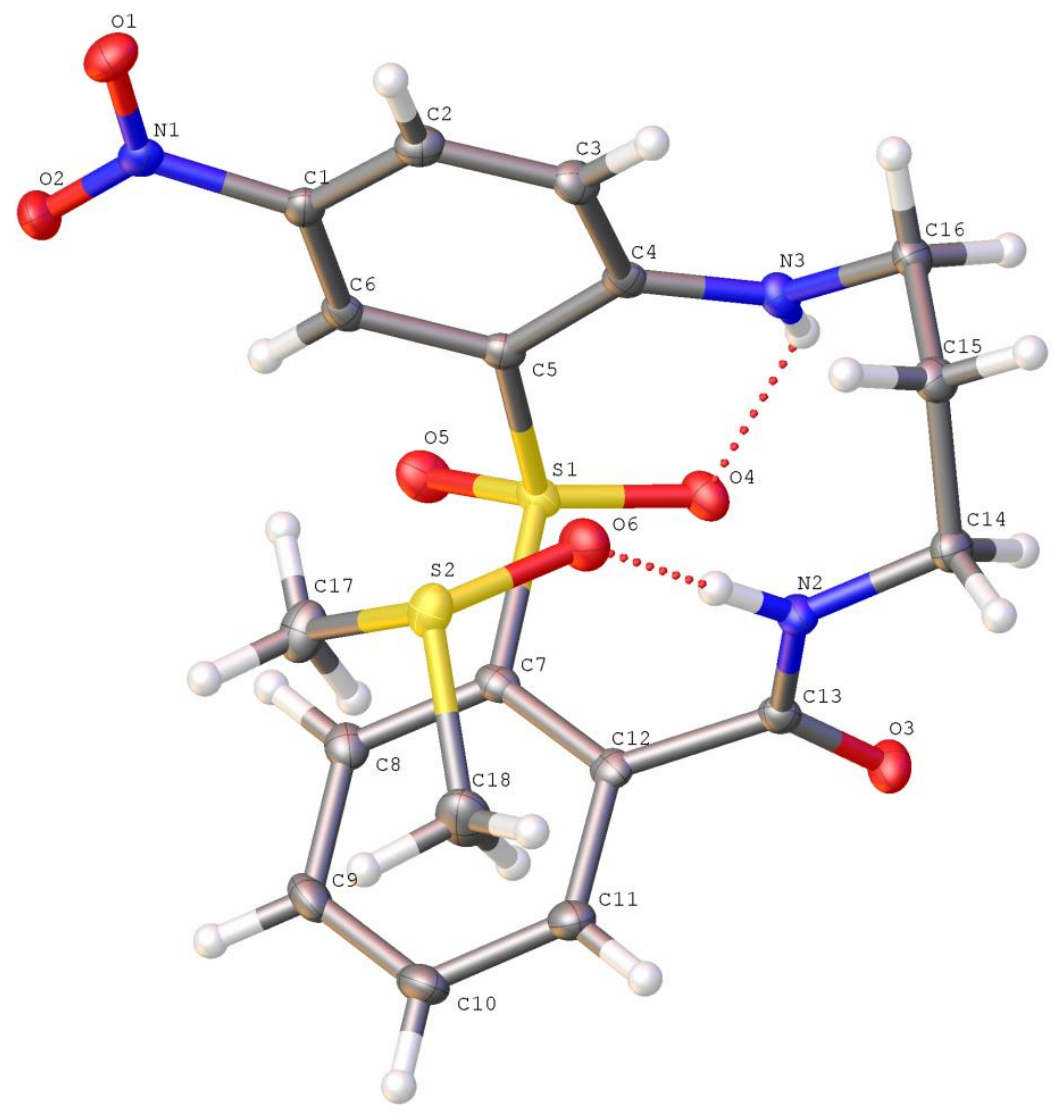

Figure S5. ORTEP representation of compound 13b displaying thermal ellipsoids at 50\% 


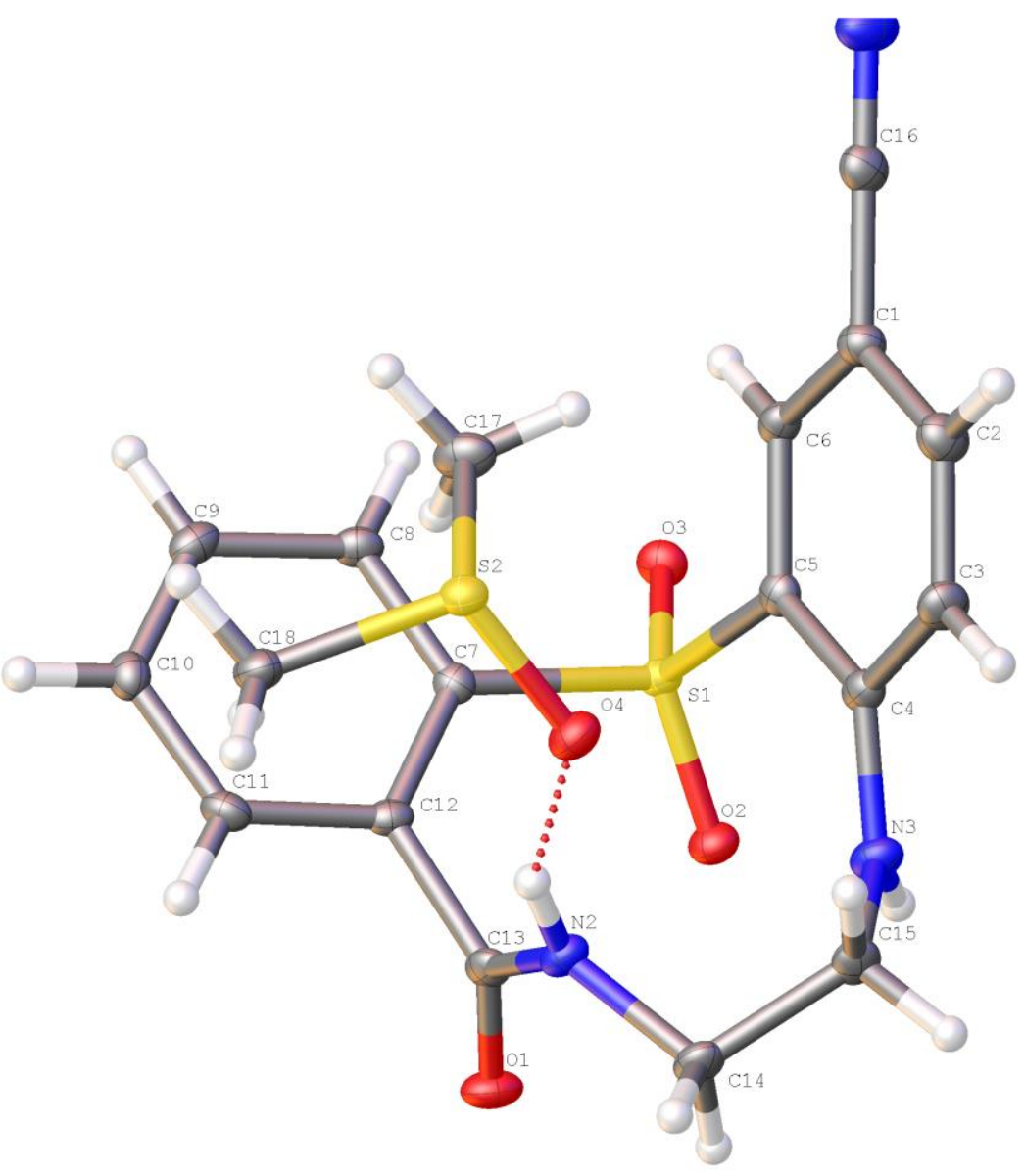

Figure S6. ORTEP representation of compound 13e displaying thermal ellipsoids at 50\%

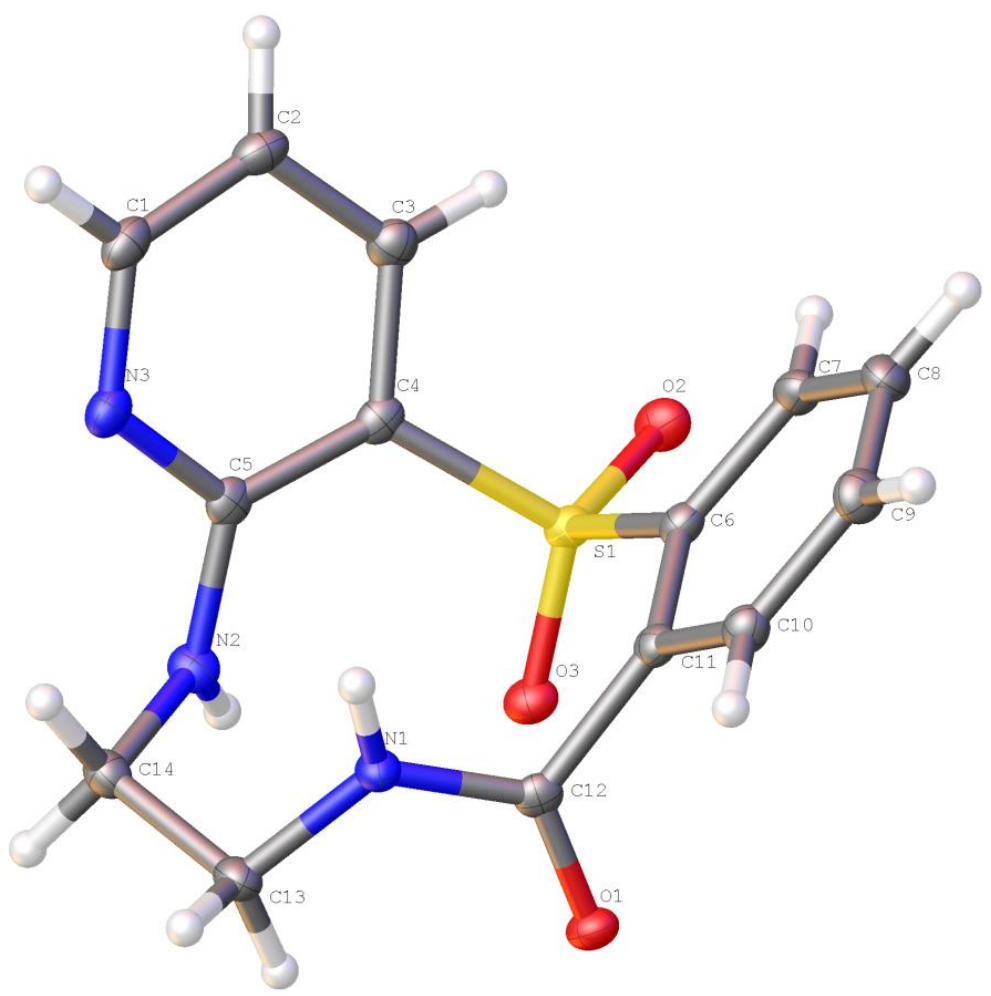

Figure S7. ORTEP representation of compound $\mathbf{1 3 f}$ displaying thermal ellipsoids at $50 \%$ 


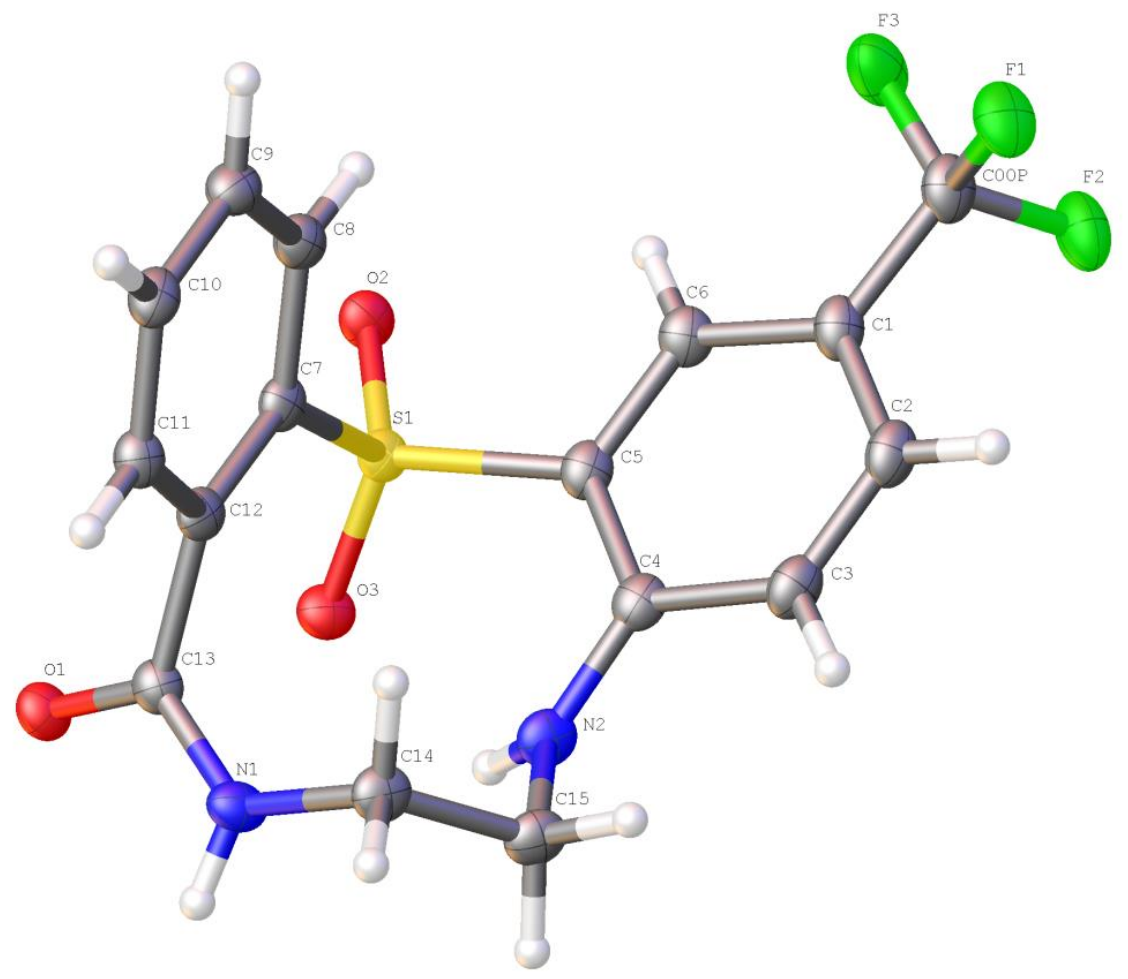

Figure S8. ORTEP representation of compound $\mathbf{1 3 h}$ displaying thermal ellipsoids at $50 \%$ 


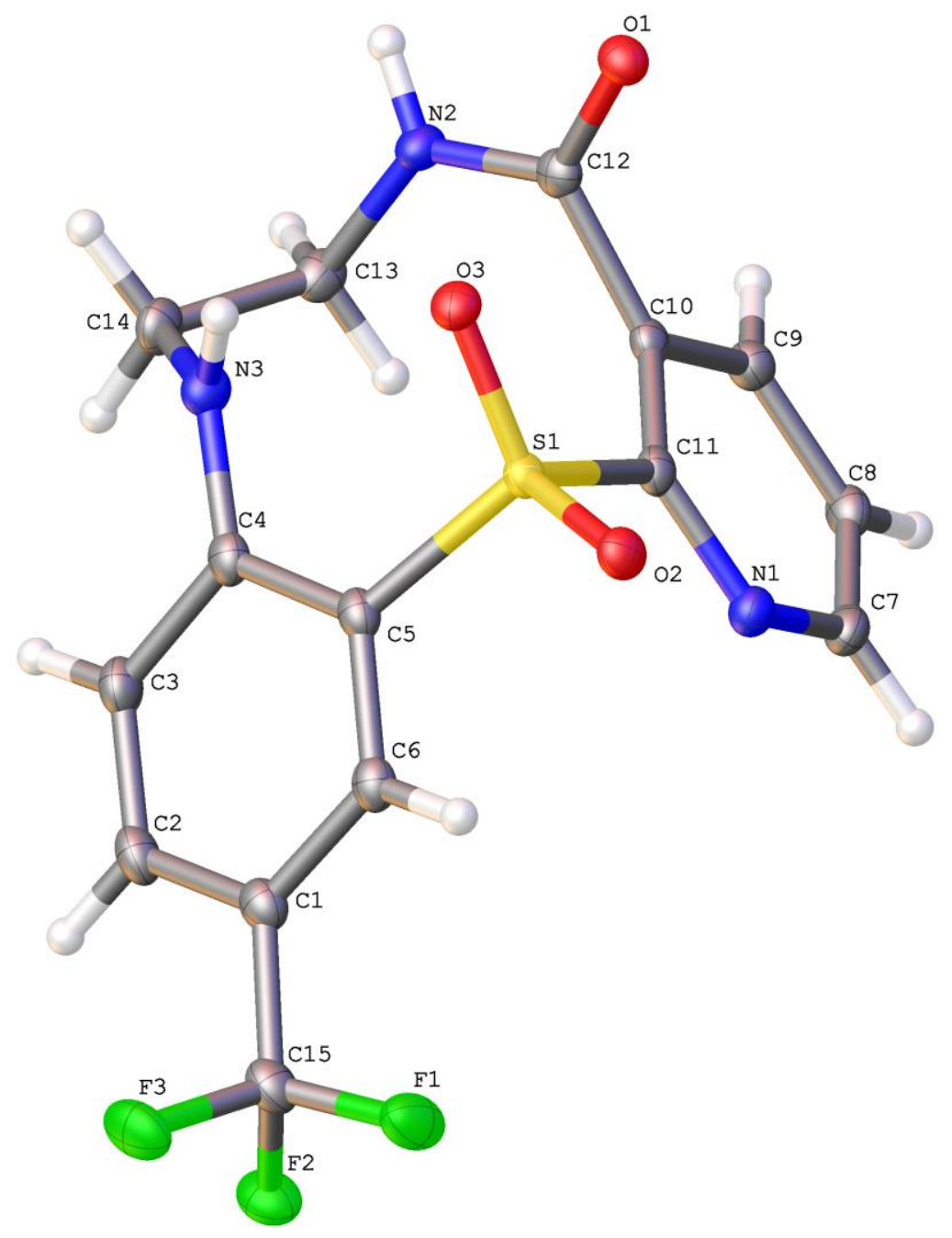

Figure S9. ORTEP representation of compound 13I displaying thermal ellipsoids at 50\% 


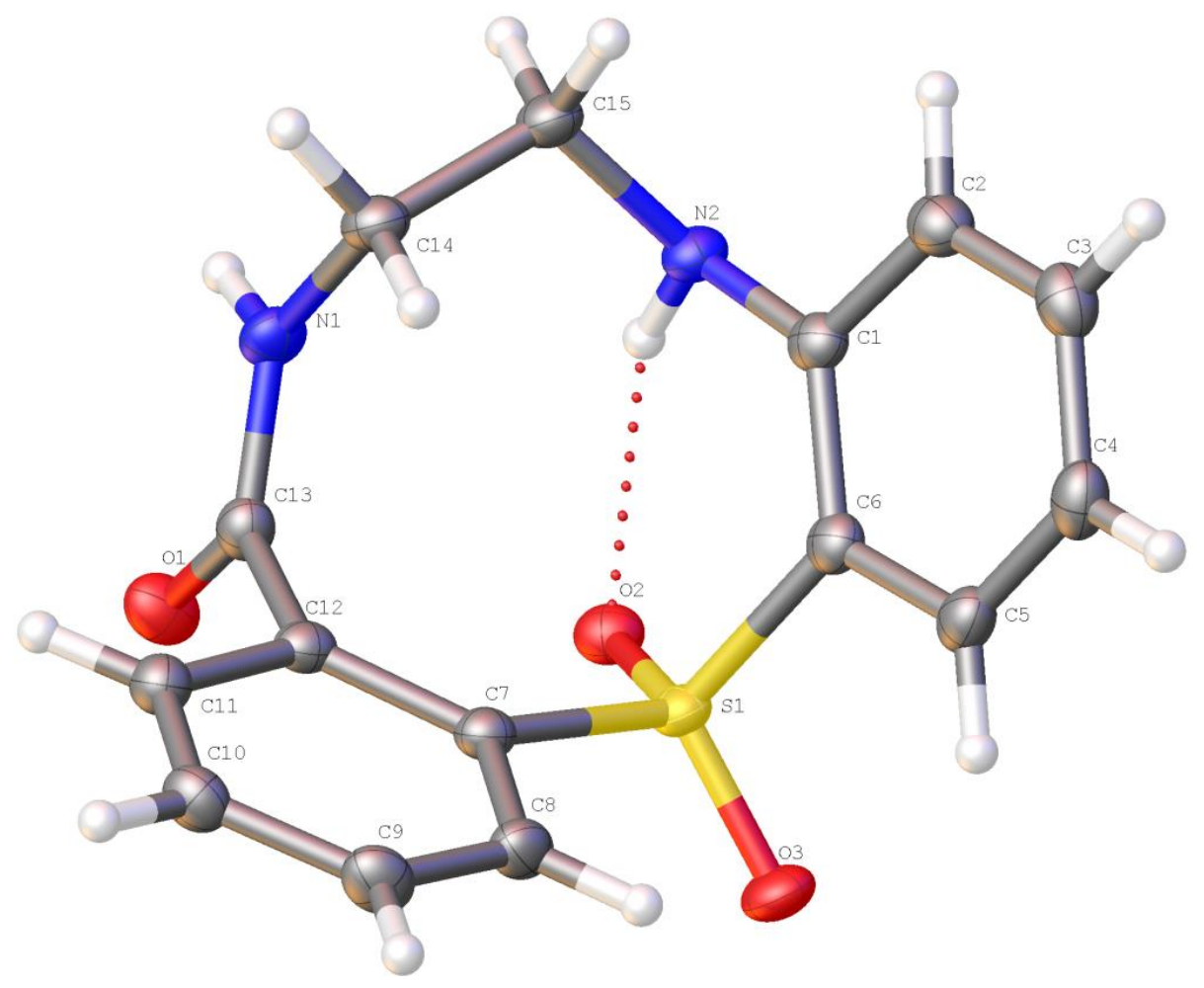

Figure S10. ORTEP representation of compound 13n displaying thermal ellipsoids at 50\%

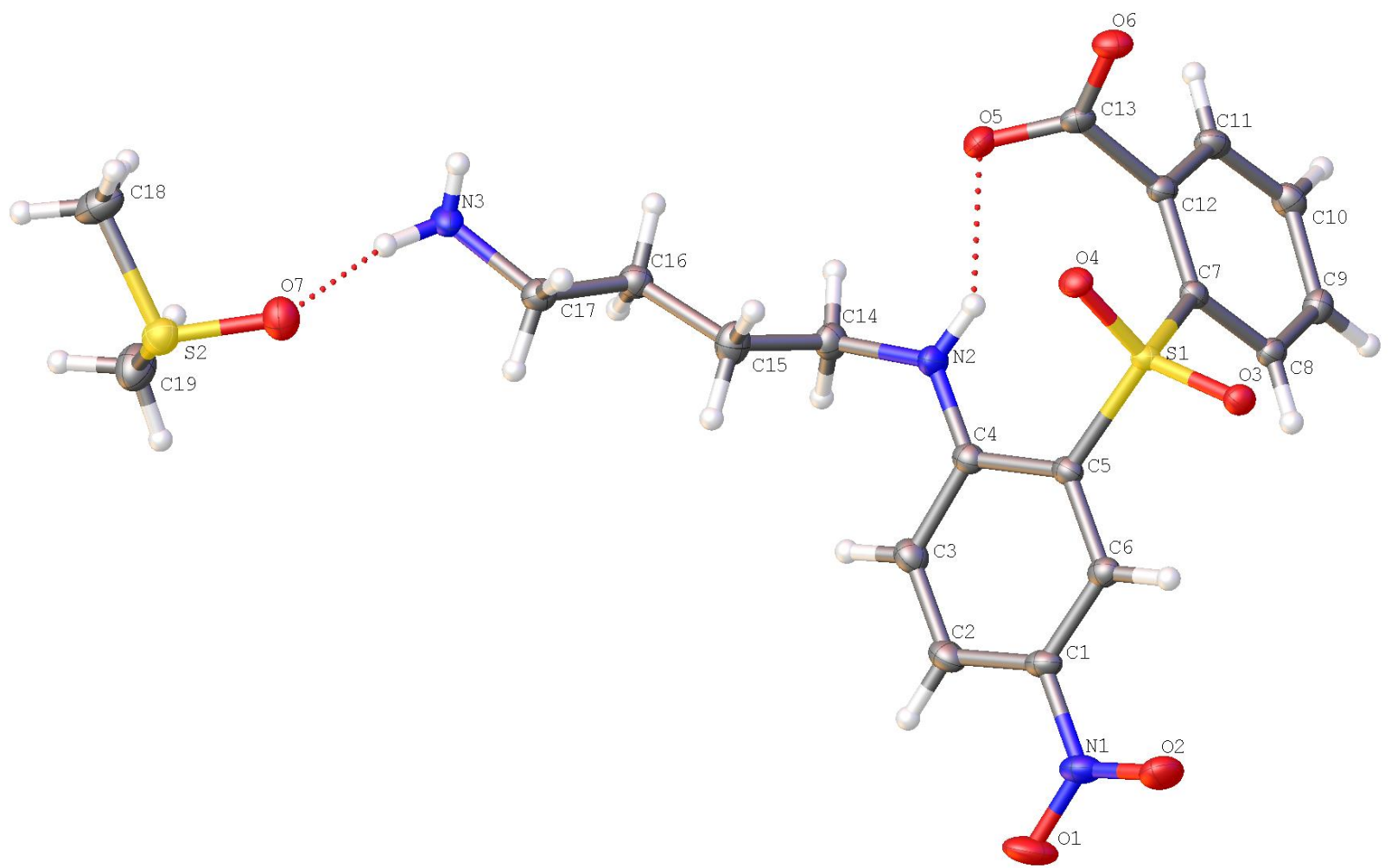

Figure S11. ORTEP representation of compound 14b displaying thermal ellipsoids at 50\% 


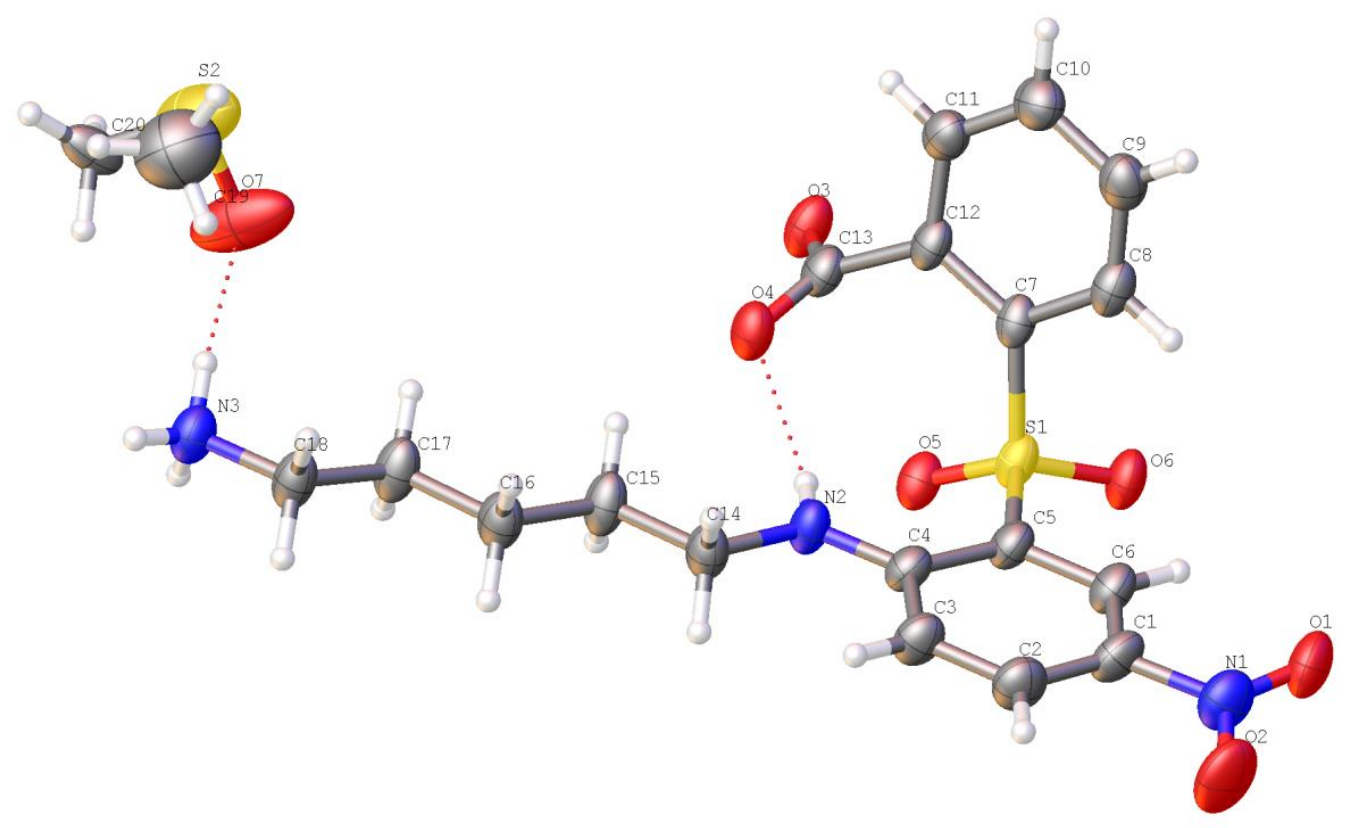

Figure S12. ORTEP representation of compound 14c displaying thermal ellipsoids at 50\% Table S1. Crystal data and structure refinement for 12a, 12e and 12n

\begin{tabular}{|c|c|c|c|}
\hline Identification code & $12 \mathrm{a}$ & $12 \mathrm{e}$ & $12 n$ \\
\hline Empirical formula & $\mathrm{C}_{15} \mathrm{H}_{13} \mathrm{~N}_{3} \mathrm{O}_{3} \mathrm{~S}$ & $\mathrm{C}_{16} \mathrm{H}_{13} \mathrm{~N}_{3} \mathrm{OS}$ & $\mathrm{C}_{15} \mathrm{H}_{14} \mathrm{~N}_{2} \mathrm{OS}$ \\
\hline Formula weight & 315.35 & 295.35 & 270.36 \\
\hline Temperature/K & 99.9(9) & $100.00(10)$ & $100(1)$ \\
\hline Crystal system & monoclinic & orthorhombic & monoclinic \\
\hline Space group & $\mathrm{P} 21 / \mathrm{n}$ & $\mathrm{P} 2{ }_{1}{ }_{1} 2_{1}$ & $\mathrm{P} 2{ }_{1}$ \\
\hline $\mathrm{a} / \AA \AA$ & $4.86340(10)$ & $9.04860(10)$ & $8.1765(2)$ \\
\hline $\mathrm{b} / \AA ̊$ & $16.2262(4)$ & $9.56180(10)$ & $9.0435(2)$ \\
\hline $\mathrm{c} / \AA \AA$ & $17.6182(4)$ & $16.2462(2)$ & $9.4429(3)$ \\
\hline$\alpha /^{\circ}$ & 90 & 90 & 90 \\
\hline$\beta /{ }^{\circ}$ & $92.370(2)$ & 90 & $109.658(3)$ \\
\hline$\gamma /{ }^{\circ}$ & 90 & 90 & 90 \\
\hline Volume $/ \AA^{3}$ & $1389.14(6)$ & 1405.64(3) & $657.55(3)$ \\
\hline $\mathrm{Z}$ & 4 & 4 & 2 \\
\hline pcalcg $/ \mathrm{cm}^{3}$ & 1.5077 & 1.396 & 1.3654 \\
\hline
\end{tabular}




\begin{tabular}{|c|c|c|c|}
\hline$\mu / \mathrm{mm}^{-1}$ & 2.234 & 2.060 & 2.123 \\
\hline $\mathrm{F}(000)$ & 659.4 & 616.0 & 285.4 \\
\hline Crystal size $/ \mathrm{mm}_{3}$ & $0.43 \times 0.34 \times 0.21$ & $0.32 \times 0.21 \times 0.1$ & $0.42 \times 0.36 \times 0.24$ \\
\hline Radiation & $\mathrm{Cu} \mathrm{K} \alpha(\lambda=1.54184)$ & $\mathrm{CuK} \alpha(\lambda=1.54184)$ & $\mathrm{Cu} \mathrm{K} \alpha(\lambda=1.54184)$ \\
\hline $2 \Theta$ range for data collection ${ }^{\circ}$ & 7.4 to 141 & 10.736 to 154.608 & 9.94 to 141.44 \\
\hline Index ranges & $\begin{array}{l}-5 \leq \mathrm{h} \leq 5,-19 \leq \mathrm{k} \leq 19,- \\
21 \leq 1 \leq 21\end{array}$ & $\begin{array}{l}-11 \leq \mathrm{h} \leq 11,-12 \leq \mathrm{k} \leq 11,- \\
20 \leq 1 \leq 18\end{array}$ & $\begin{array}{l}-9 \leq \mathrm{h} \leq 9,-11 \leq \mathrm{k} \leq 11 \\
-11 \leq 1 \leq 11\end{array}$ \\
\hline Reflections collected & 13141 & 54676 & 11085 \\
\hline Independent reflections & $\begin{array}{l}2633\left[R_{\text {int }}=0.0776\right. \\
\left.\mathrm{R}_{\text {sigma }}=0.0490\right]\end{array}$ & $\begin{array}{l}2951\left[\mathrm{R}_{\text {int }}=0.0545, \mathrm{R}_{\text {sigma }}=\right. \\
0.0157]\end{array}$ & $\begin{array}{l}2503\left[\mathrm{R}_{\text {int }}=0.0519,\right. \\
\left.\mathrm{R}_{\text {sigma }}=0.0388\right]\end{array}$ \\
\hline Data/restraints/parameters & $2633 / 0 / 199$ & $2951 / 0 / 190$ & $2503 / 1 / 172$ \\
\hline Goodness-of-fit on $\mathrm{F}_{2}$ & 1.026 & 1.040 & 1.048 \\
\hline Final $\mathrm{R}$ indexes $[\mathrm{I}>=2 \sigma(\mathrm{I})]$ & $\begin{array}{l}\mathrm{R}_{1}=0.0466, \mathrm{wR}_{2}= \\
0.1212\end{array}$ & $\mathrm{R}_{1}=0.0281, w \mathrm{R}_{2}=0.0738$ & $\begin{array}{l}\mathrm{R}_{1}=0.0356, \mathrm{wR}_{2}= \\
0.0880\end{array}$ \\
\hline Final $\mathrm{R}$ indexes [all data] & $\begin{array}{l}\mathrm{R}_{1}=0.0542, \mathrm{wR}_{2}= \\
0.1299\end{array}$ & $\mathrm{R}_{1}=0.0283, \mathrm{w}_{2}=0.0740$ & $\begin{array}{l}\mathrm{R}_{1}=0.0379, \mathrm{wR}_{2}= \\
0.0914\end{array}$ \\
\hline Largest diff. peak/hole / e $\AA^{-3}$ & $0.57 /-0.40$ & $0.43 /-0.46$ & $0.49 /-0.48$ \\
\hline CCDC & 2046971 & 2046972 & 2046970 \\
\hline
\end{tabular}

Table S2. Crystal data and structure refinement for $\mathbf{1 3 a}, \mathbf{1 3 b}$ and $13 \mathbf{e}$

\begin{tabular}{|l|l|l|l|}
\hline Identification code & $\mathbf{1 3 a}$ & $\mathbf{1 3 b}$ & $\mathbf{1 3 e}$ \\
\hline Empirical formula & $\mathrm{C}_{32} \mathrm{H}_{32} \mathrm{~N}_{6} \mathrm{O}_{11} \mathrm{~S}_{3}$ & $\mathrm{C}_{18} \mathrm{H}_{22} \mathrm{~N}_{3} \mathrm{O}_{6} \mathrm{~S}_{2}$ & $\mathrm{C}_{18} \mathrm{H}_{19} \mathrm{~N}_{3} \mathrm{O}_{4} \mathrm{~S}_{2}$ \\
\hline Formula weight & 772.81 & 439.52 & 405.48 \\
\hline Temperature/K & $100.02(11)$ & $100.0(6)$ & $100.00(10)$ \\
\hline Crystal system & triclinic & monoclinic & monoclinic \\
\hline Space group & $\mathrm{P}-1$ & $\mathrm{P} 2{ }_{1} / \mathrm{c}$ & $\mathrm{P} 2{ }_{1} / \mathrm{c}$ \\
\hline $\mathrm{a} / \AA$ & $7.75190(10)$ & $15.7318(2)$ & $14.3903(3)$ \\
\hline $\mathrm{b} / \AA$ & $15.5891(2)$ & $8.86940(10)$ & $8.8407(2)$ \\
\hline $\mathrm{c} / \AA$ & $15.8319(2)$ & $14.9398(2)$ & $14.3448(3)$ \\
\hline$\alpha /{ }^{\circ}$ & $63.108(2)$ & 90 & 90 \\
\hline$\beta /{ }^{\circ}$ & $88.8610(10)$ & $111.2940(10)$ & $96.437(2)$ \\
\hline
\end{tabular}




\begin{tabular}{|c|c|c|c|}
\hline$\gamma /{ }^{\circ}$ & $77.9050(10)$ & 90 & 90 \\
\hline Volume $/ \AA^{3}$ & $1662.27(5)$ & $1942.26(4)$ & $1813.45(7)$ \\
\hline $\mathrm{Z}$ & 2 & 4 & 4 \\
\hline$\rho c a l c g / \mathrm{cm}^{3}$ & 1.544 & 1.5029 & 1.485 \\
\hline$\mu / \mathrm{mm}^{-1}$ & 2.670 & 2.866 & 2.935 \\
\hline $\mathrm{F}(000)$ & 804.0 & 925.6 & 848.0 \\
\hline Crystal size/mm 3 & $0.6 \times 0.4 \times 0.3$ & $0.35 \times 0.23 \times 0.19$ & $0.34 \times 0.2 \times 0.12$ \\
\hline Radiation & $\operatorname{CuK} \alpha(\lambda=1.54184)$ & $\mathrm{Cu} \mathrm{K} \alpha(\lambda=1.54184)$ & $\mathrm{CuK} \alpha(\lambda=1.54184)$ \\
\hline $2 \Theta$ range for data collection $/^{\circ}$ & 6.282 to 141.022 & 6.02 to 140.86 & 6.18 to 151.75 \\
\hline Index ranges & $\begin{array}{l}-9 \leq \mathrm{h} \leq 9,-19 \leq \mathrm{k} \leq 18,- \\
19 \leq 1 \leq 19\end{array}$ & $\begin{array}{l}-16 \leq \mathrm{h} \leq 19,-10 \leq \mathrm{k} \leq 10,- \\
18 \leq 1 \leq 18\end{array}$ & $\begin{array}{l}-16 \leq \mathrm{h} \leq 17,-10 \leq \mathrm{k} \leq \\
11,-18 \leq 1 \leq 18\end{array}$ \\
\hline Reflections collected & 36618 & 22272 & 10348 \\
\hline Independent reflections & $\begin{array}{l}6339\left[\mathrm{R}_{\text {int }}=0.0472,\right. \\
\left.\mathrm{R}_{\text {sigma }}=0.0288\right]\end{array}$ & $\begin{array}{l}3703\left[\mathrm{R}_{\text {int }}=0.0469, \mathrm{R}_{\text {sigma }}=\right. \\
0.0276]\end{array}$ & $\begin{array}{l}3720\left[R_{\text {int }}=0.0271,\right. \\
\left.R_{\text {sigma }}=0.0284\right]\end{array}$ \\
\hline Data/restraints/parameters & $6339 / 0 / 471$ & $3703 / 0 / 264$ & $3720 / 0 / 246$ \\
\hline Goodness-of-fit on $\mathrm{F}_{2}$ & 1.055 & 1.045 & 1.036 \\
\hline Final $\mathrm{R}$ indexes $[\mathrm{I}>=2 \sigma(\mathrm{I})]$ & $\begin{array}{l}\mathrm{R}_{1}=0.0379, \mathrm{wR}_{2}= \\
0.1050\end{array}$ & $\mathrm{R}_{1}=0.0315, \mathrm{wR}_{2}=0.0825$ & $\begin{array}{l}\mathrm{R}_{1}=0.0350, \mathrm{wR}_{2}= \\
0.0896\end{array}$ \\
\hline Final $\mathrm{R}$ indexes [all data] & $\begin{array}{l}\mathrm{R}_{1}=0.0420, \mathrm{wR}_{2}= \\
0.1087\end{array}$ & $\mathrm{R}_{1}=0.0330, \mathrm{wR}_{2}=0.0837$ & $\begin{array}{l}\mathrm{R}_{1}=0.0403, \mathrm{wR}_{2}= \\
0.0940\end{array}$ \\
\hline Largest diff. peak/hole / e $\AA^{-3}$ & $0.49 /-0.45$ & $0.38 /-0.47$ & $0.61 /-0.58$ \\
\hline CCDC & 2046962 & 2046969 & 2046965 \\
\hline
\end{tabular}

Table S3. Crystal data and structure refinement for $\mathbf{1 3 f}, \mathbf{1 3 h}$ and $\mathbf{1 3 l}$

\begin{tabular}{|l|l|l|l|}
\hline Identification code & $\mathbf{1 3 f}$ & $\mathbf{1 3 h}$ & $\mathbf{1 3 I}$ \\
\hline Empirical formula & $\mathrm{C}_{14} \mathrm{H}_{13} \mathrm{~N}_{3} \mathrm{O}_{3} \mathrm{~S}$ & $\mathrm{C}_{16} \mathrm{H}_{13} \mathrm{~F}_{3} \mathrm{~N}_{2} \mathrm{O}_{3} \mathrm{~S}$ & $\mathrm{C}_{15} \mathrm{H}_{12} \mathrm{~F}_{3} \mathrm{~N}_{3} \mathrm{O}_{3} \mathrm{~S}$ \\
\hline Formula weight & 303.33 & 370.34 & 371.34 \\
\hline Temperature/K & $99.98(10)$ & $100.00(10)$ & $100.00(10)$ \\
\hline Crystal system & orthorhombic & monoclinic & monoclinic \\
\hline Space group & $\mathrm{P} 2{ }_{1} 22_{1}$ & $\mathrm{P} 2{ }_{1} / \mathrm{n}$ & $\mathrm{P} 2{ }_{1} / \mathrm{n}$ \\
\hline $\mathrm{a} / \AA$ & $8.51550(10)$ & $9.70820(10)$ & $9.6283(3)$ \\
\hline
\end{tabular}




\begin{tabular}{|c|c|c|c|}
\hline $\mathrm{b} / \AA$ & $9.28550(10)$ & $11.7410(2)$ & $11.4639(3)$ \\
\hline $\mathrm{c} / \AA$ & $16.5383(2)$ & $13.3427(2)$ & $13.2598(3)$ \\
\hline$\alpha /^{\circ}$ & 90 & 90 & 90 \\
\hline$\beta /{ }^{\circ}$ & 90 & $93.3700(10)$ & $93.741(2)$ \\
\hline$\gamma /{ }^{\circ}$ & 90 & 90 & 90 \\
\hline Volume $/ \AA^{3}$ & $1307.69(3)$ & $1518.22(4)$ & $1460.47(7)$ \\
\hline $\mathrm{Z}$ & 4 & 4 & 4 \\
\hline$\rho c a l c g / \mathrm{cm}^{3}$ & 1.541 & 1.620 & 1.689 \\
\hline$\mu / \mathrm{mm}^{-1}$ & 2.346 & 2.416 & 2.534 \\
\hline $\mathrm{F}(000)$ & 632.0 & 760.0 & 760.0 \\
\hline Crystal size $/ \mathrm{mm}_{3}$ & $0.32 \times 0.18 \times 0.15$ & $0.32 \times 0.23 \times 0.13$ & $0.26 \times 0.2 \times 0.18$ \\
\hline Radiation & $\mathrm{CuK} \alpha(\lambda=1.54184)$ & $\mathrm{CuK} \alpha(\lambda=1.54184)$ & $\mathrm{CuK} \alpha(\lambda=1.54184)$ \\
\hline $2 \Theta$ range for data collection $/^{\circ}$ & 10.698 to 154.792 & 10.042 to 140.99 & 10.21 to 154.728 \\
\hline Index ranges & $\begin{array}{l}-10 \leq \mathrm{h} \leq 10,-11 \leq \mathrm{k} \leq \\
11,-20 \leq 1 \leq 18\end{array}$ & $\begin{array}{l}-11 \leq \mathrm{h} \leq 10,-14 \leq \mathrm{k} \leq 14,- \\
16 \leq 1 \leq 16\end{array}$ & $\begin{array}{l}-11 \leq \mathrm{h} \leq 12,-14 \leq \mathrm{k} \leq \\
14,-13 \leq 1 \leq 16\end{array}$ \\
\hline Reflections collected & 48540 & 15202 & 12277 \\
\hline Independent reflections & $\begin{array}{l}2740\left[\mathrm{R}_{\text {int }}=0.0502,\right. \\
\left.\mathrm{R}_{\text {sigma }}=0.0156\right]\end{array}$ & $\begin{array}{l}2902\left[\mathrm{R}_{\text {int }}=0.0441, \mathrm{R}_{\text {sigma }}=\right. \\
0.0311]\end{array}$ & $\begin{array}{l}3036\left[R_{\text {int }}=0.0337,\right. \\
\left.R_{\text {sigma }}=0.0274\right]\end{array}$ \\
\hline Data/restraints/parameters & $2740 / 0 / 190$ & $2902 / 0 / 226$ & $3036 / 0 / 226$ \\
\hline Goodness-of-fit on $\mathrm{F}_{2}$ & 1.061 & 1.080 & 1.057 \\
\hline Final $\mathrm{R}$ indexes $[\mathrm{I}>=2 \sigma(\mathrm{I})]$ & $\begin{array}{l}\mathrm{R}_{1}=0.0244, \mathrm{wR}_{2}= \\
0.0655\end{array}$ & $\mathrm{R}_{1}=0.0354, w \mathrm{R}_{2}=0.0962$ & $\begin{array}{l}\mathrm{R}_{1}=0.0364, \mathrm{wR}_{2}= \\
0.0948\end{array}$ \\
\hline Final $\mathrm{R}$ indexes [all data] & $\begin{array}{l}\mathrm{R}_{1}=0.0246, \mathrm{wR}_{2}= \\
0.0657\end{array}$ & $\mathrm{R}_{1}=0.0379, \mathrm{wR}_{2}=0.0985$ & $\begin{array}{l}\mathrm{R}_{1}=0.0376, \mathrm{wR}_{2}= \\
0.0959\end{array}$ \\
\hline Largest diff. peak/hole / e $\AA^{-3}$ & $0.34 /-0.34$ & $0.36 /-0.45$ & $0.48 /-0.52$ \\
\hline CCDC & 2046974 & 2046968 & 2046963 \\
\hline
\end{tabular}

Table S4. Crystal data and structure refinement for $\mathbf{1 3 a}, \mathbf{1 3 b}$ and $13 \mathbf{e}$

\begin{tabular}{|l|l|l|l|}
\hline Identification code & $\mathbf{1 3 n}$ & $\mathbf{1 4 b}$ & $\mathbf{1 4 c}$ \\
\hline Empirical formula & $\mathrm{C}_{15} \mathrm{H}_{14} \mathrm{~N}_{2} \mathrm{O}_{3} \mathrm{~S}$ & $\mathrm{C}_{19} \mathrm{H}_{24} \mathrm{~N}_{3} \mathrm{O}_{7} \mathrm{~S}_{2}$ & $\mathrm{C}_{20} \mathrm{H}_{27} \mathrm{~N}_{3} \mathrm{O}_{7} \mathrm{~S}_{2}$ \\
\hline Formula weight & 302.34 & 470.53 & 485.56 \\
\hline
\end{tabular}




\begin{tabular}{|c|c|c|c|}
\hline Temperature/K & $100.00(10)$ & $100.00(16)$ & $100.0(7)$ \\
\hline Crystal system & orthorhombic & monoclinic & triclinic \\
\hline Space group & Pbca & $\mathrm{P} 2{ }_{1} / \mathrm{c}$ & P-1 \\
\hline $\mathrm{a} / \AA$ & $16.1093(2)$ & $8.48590(10)$ & $8.8017(5)$ \\
\hline $\mathrm{b} / \AA$ & $9.86360(10)$ & $9.5748(2)$ & $11.1423(5)$ \\
\hline $\mathrm{c} / \AA$ & $17.0435(2)$ & $26.5591(4)$ & $12.6895(5)$ \\
\hline$\alpha / /^{\circ}$ & 90 & 90 & $68.119(4)$ \\
\hline$\beta /{ }^{\circ}$ & 90 & $93.1670(10)$ & $79.303(4)$ \\
\hline$\gamma^{\circ}$ & 90 & 90 & $84.208(4)$ \\
\hline Volume $/ \AA^{3}$ & $2708.14(5)$ & $2154.65(6)$ & 1134.07(10) \\
\hline $\bar{Z}$ & 8 & 4 & 2 \\
\hline$\rho c a l c g / \mathrm{cm}^{3}$ & 1.483 & 1.451 & 1.422 \\
\hline$\mu / \mathrm{mm}^{-1}$ & 2.241 & 2.657 & 2.540 \\
\hline $\mathrm{F}(000)$ & 1264.0 & 988.0 & 512.0 \\
\hline Crystal size $/ \mathrm{mm}_{3}$ & $0.32 \times 0.27 \times 0.19$ & $0.21 \times 0.2 \times 0.13$ & $0.3 \times 0.22 \times 0.12$ \\
\hline Radiation & $\mathrm{CuK} \alpha(\lambda=1.54184)$ & $\mathrm{CuK} \alpha(\lambda=1.54184)$ & $\mathrm{CuK} \alpha(\lambda=1.54184)$ \\
\hline $2 \Theta$ range for data collection $/^{\circ}$ & 10.38 to 154.882 & 6.666 to 154.906 & 7.606 to 144.83 \\
\hline Index ranges & $\begin{array}{l}-20 \leq \mathrm{h} \leq 20,-12 \leq \mathrm{k} \leq \\
12,-17 \leq 1 \leq 21\end{array}$ & $\begin{array}{l}-9 \leq \mathrm{h} \leq 10,-12 \leq \mathrm{k} \leq 11,-33 \\
\leq 1 \leq 33\end{array}$ & $\begin{array}{l}-10 \leq \mathrm{h} \leq 10,-13 \leq \mathrm{k} \leq \\
13,-15 \leq 1 \leq 15\end{array}$ \\
\hline Reflections collected & 51077 & 24697 & 19539 \\
\hline Independent reflections & $\begin{array}{l}2861\left[R_{\text {int }}=0.0382,\right. \\
\left.R_{\text {sigma }}=0.0145\right]\end{array}$ & $\begin{array}{l}4468\left[R_{\text {int }}=0.0340, R_{\text {sigma }}=\right. \\
0.0231]\end{array}$ & $\begin{array}{l}4327\left[R_{\text {int }}=0.1091,\right. \\
\left.R_{\text {sigma }}=0.0608\right]\end{array}$ \\
\hline Data/restraints/parameters & $2861 / 0 / 190$ & $4468 / 0 / 288$ & $4327 / 0 / 292$ \\
\hline Goodness-of-fit on $\mathrm{F}_{2}$ & 1.070 & 1.068 & 1.880 \\
\hline Final $\mathrm{R}$ indexes $[\mathrm{I}>=2 \sigma(\mathrm{I})]$ & $\begin{array}{l}\mathrm{R}_{1}=0.0332, \mathrm{w} \mathrm{R}_{2}= \\
0.0857\end{array}$ & $\mathrm{R}_{1}=0.0411, w \mathrm{R}_{2}=0.1202$ & $\begin{array}{l}\mathrm{R}_{1}=0.1343, \mathrm{wR}_{2}= \\
0.3813\end{array}$ \\
\hline Final $\mathrm{R}$ indexes [all data] & $\begin{array}{l}\mathrm{R}_{1}=0.0339, \mathrm{wR}_{2}= \\
0.0862\end{array}$ & $\mathrm{R}_{1}=0.0431, \mathrm{w} \mathrm{R}_{2}=0.1220$ & $\begin{array}{l}\mathrm{R}_{1}=0.1541, \mathrm{wR}_{2}= \\
0.4380\end{array}$ \\
\hline Largest diff. peak/hole / e $\AA^{-3}$ & $0.27 /-0.39$ & $1.08 /-0.84$ & $1.79 /-1.84$ \\
\hline CCDC & 2046967 & 2046975 & 2046976 \\
\hline
\end{tabular}


III. Example of kobs calculation (compound 9a)

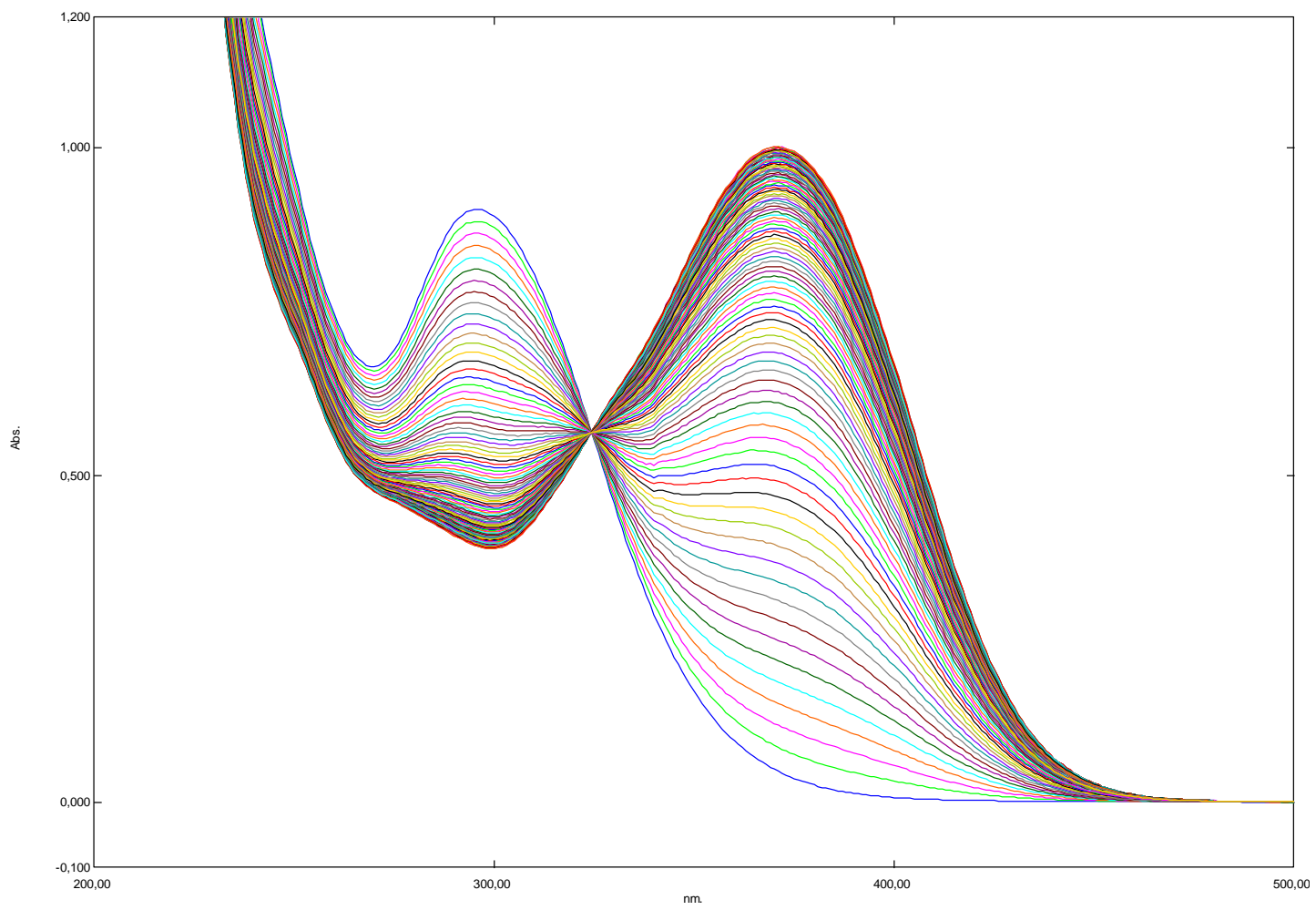

Figure S13. UV-spectrum of the ring expansion reaction of compound 9a

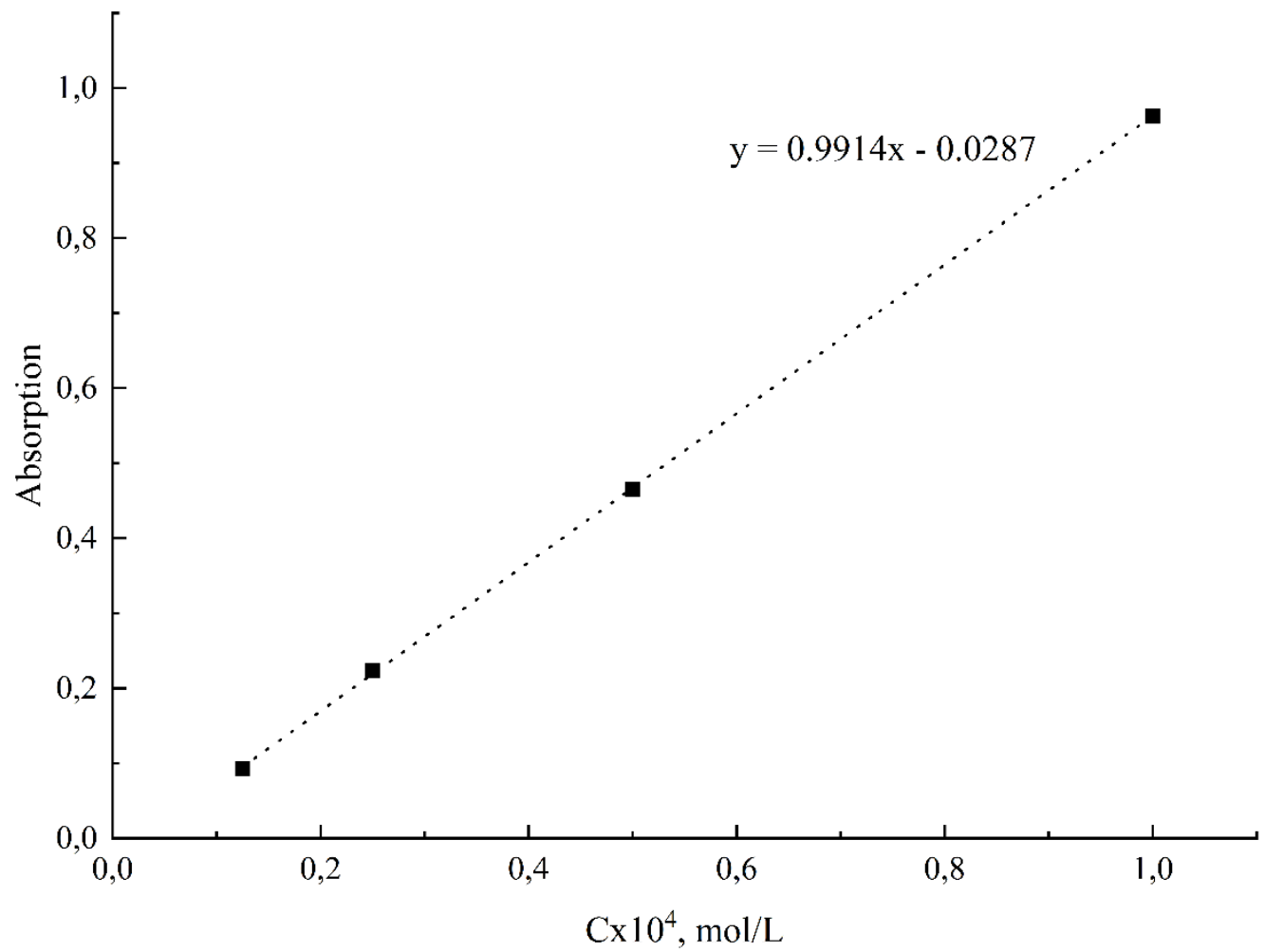

Figure S14. Calibration graph of the dependence of the adsorption of solutions of compaund 9a at various concentrations 
Table S5. Calculated values of the concentration of compound 9a in the reaction mass at different times

\begin{tabular}{|c|c|c|}
\hline Time, min & $\begin{array}{c}\text { Absorption } \\
\text { (wavelength } 291 \mathrm{~nm} \text { ) }\end{array}$ & $\begin{array}{c}\text { Concentration, } \\
\mathrm{mol} / \mathrm{L} * 10^{4}\end{array}$ \\
\hline 0 & 0,892 & 0,929 \\
\hline 10 & 0,875 & 0,912 \\
\hline 20 & 0,857 & 0,893 \\
\hline 30 & 0,84 & 0,876 \\
\hline 40 & 0,822 & 0,858 \\
\hline 50 & 0,805 & 0,841 \\
\hline 60 & 0,789 & 0,825 \\
\hline 70 & 0,772 & 0,808 \\
\hline 80 & 0,756 & 0,792 \\
\hline 90 & 0,741 & 0,776 \\
\hline 100 & 0,726 & 0,761 \\
\hline 110 & 0,711 & 0,746 \\
\hline 120 & 0,698 & 0,733 \\
\hline 130 & 0,685 & 0,720 \\
\hline 140 & 0,672 & 0,707 \\
\hline 150 & 0,659 & 0,694 \\
\hline 160 & 0,648 & 0,683 \\
\hline 170 & 0,637 & 0,671 \\
\hline 180 & 0,626 & 0,660 \\
\hline 190 & 0,616 & 0,650 \\
\hline 200 & 0,606 & 0,640 \\
\hline 210 & 0,597 & 0,631 \\
\hline 220 & 0,588 & 0,622 \\
\hline 230 & 0,58 & 0,614 \\
\hline 240 & 0,573 & 0,607 \\
\hline 250 & 0,565 & 0,599 \\
\hline 260 & 0,558 & 0,592 \\
\hline 270 & 0,551 & 0,585 \\
\hline 280 & 0,545 & 0,579 \\
\hline 290 & 0,539 & 0,573 \\
\hline
\end{tabular}




\begin{tabular}{|c|c|c|}
\hline 300 & 0,533 & 0,567 \\
\hline 310 & 0,528 & 0,562 \\
\hline 320 & 0,523 & 0,556 \\
\hline 330 & 0,518 & 0,551 \\
\hline 340 & 0,513 & 0,546 \\
\hline 350 & 0,508 & 0,541 \\
\hline 360 & 0,504 & 0,537 \\
\hline 370 & 0,5 & 0,533 \\
\hline 380 & 0,496 & 0,529 \\
\hline 390 & 0,492 & 0,525 \\
\hline 400 & 0,489 & 0,522 \\
\hline 410 & 0,485 & 0,518 \\
\hline 420 & 0,482 & 0,515 \\
\hline 430 & 0,479 & 0,512 \\
\hline 440 & 0,475 & 0,508 \\
\hline 450 & 0,472 & 0,505 \\
\hline 460 & 0,469 & 0,502 \\
\hline 470 & 0,467 & 0,500 \\
\hline 480 & 0,464 & 0,497 \\
\hline 490 & 0,461 & 0,494 \\
\hline 500 & 0,459 & 0,492 \\
\hline 510 & 0,456 & 0,489 \\
\hline 520 & 0,454 & 0,487 \\
\hline 530 & 0,452 & 0,485 \\
\hline 540 & 0,449 & 0,482 \\
\hline 550 & 0,447 & 0,480 \\
\hline 560 & 0,445 & 0,478 \\
\hline 570 & 0,444 & 0,477 \\
\hline 580 & 0,442 & 0,475 \\
\hline 590 & 0,44 & 0,473 \\
\hline 600 & 0,438 & 0,471 \\
\hline 610 & 0,437 & 0,470 \\
\hline 620 & 0,435 & 0,468 \\
\hline
\end{tabular}




\begin{tabular}{|c|c|c|}
\hline 630 & 0,433 & 0,466 \\
\hline 640 & 0,432 & 0,465 \\
\hline 650 & 0,431 & 0,464 \\
\hline 660 & 0,429 & 0,462 \\
\hline 670 & 0,428 & 0,461 \\
\hline 680 & 0,427 & 0,460 \\
\hline 690 & 0,425 & 0,458 \\
\hline 700 & 0,424 & 0,457 \\
\hline 710 & 0,423 & 0,456 \\
\hline 720 & 0,422 & 0,455 \\
\hline 730 & 0,421 & 0,454 \\
\hline 740 & 0,42 & 0,453 \\
\hline 750 & 0,419 & 0,452 \\
\hline 760 & 0,418 & 0,451 \\
\hline 770 & 0,417 & 0,450 \\
\hline 780 & 0,417 & 0,450 \\
\hline 790 & 0,416 & 0,449 \\
\hline 800 & 0,415 & 0,448 \\
\hline 810 & 0,414 & 0,447 \\
\hline 820 & 0,414 & 0,447 \\
\hline 830 & 0,413 & 0,446 \\
\hline 840 & 0,412 & 0,445 \\
\hline 850 & 0,411 & 0,444 \\
\hline 860 & 0,411 & 0,444 \\
\hline 870 & 0,41 & 0,443 \\
\hline 880 & 0,41 & 0,443 \\
\hline 890 & 0,409 & 0,441 \\
\hline 900 & 0,408 & 0,440 \\
\hline 910 & 0,408 & 0,440 \\
\hline 920 & 0,407 & 0,439 \\
\hline 930 & 0,407 & 0,439 \\
\hline 940 & 0,406 & 0,438 \\
\hline 950 & 0,406 & 0,438 \\
\hline
\end{tabular}




\begin{tabular}{|l|l|l|}
960 & 0,405 & 0,437 \\
\hline 970 & 0,405 & 0,437 \\
\hline 980 & 0,404 & 0,436 \\
\hline 990 & 0,404 & 0,436 \\
\hline
\end{tabular}

Calculation of kobs for substrate 9a:

First-order kinetics equation:

$$
\frac{d \mathrm{C}}{d \mathrm{t}}=\mathrm{k}_{o b s} \cdot \mathrm{C}
$$

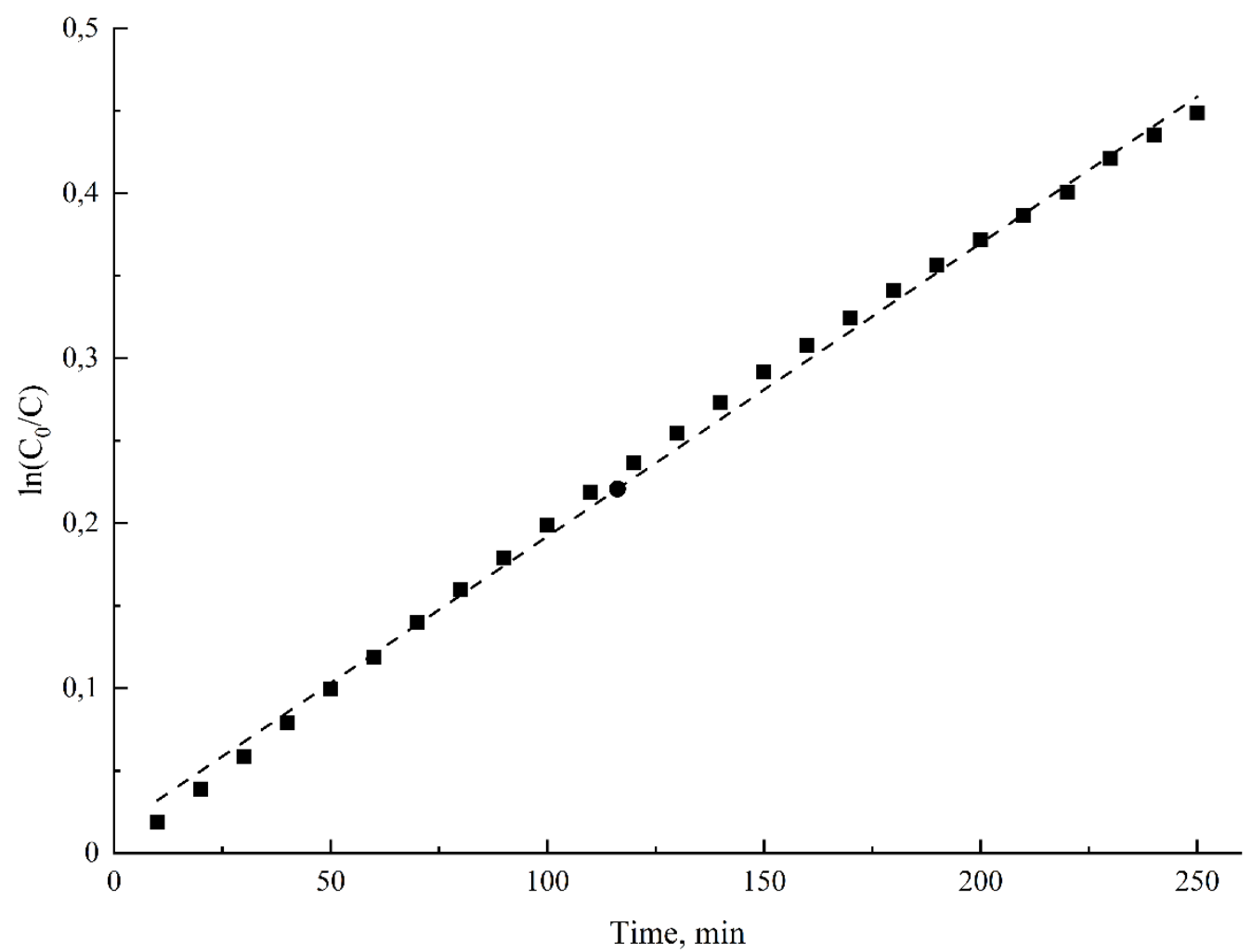

Figure S15. Linear dependence of the $\ln \left(\mathrm{C}_{0} / \mathrm{C}\right)$ on the reaction time for compound $9 \mathrm{a}$

Hence, $\mathrm{K}_{\text {obs }}=\operatorname{tg}(\alpha)=18 \times 10^{4} \mathrm{~s}^{-1}$ 
${ }^{1} \mathrm{H}$ (400 MHz; in DMSO- $\left.d_{6}\right)$ and ${ }^{13} \mathrm{C}\left\{{ }^{1} \mathrm{H}\right\}\left(101 \mathrm{MHz}\right.$; in DMSO- $\left.d_{6}\right)$ NMR spectra of compound Ba

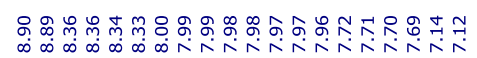
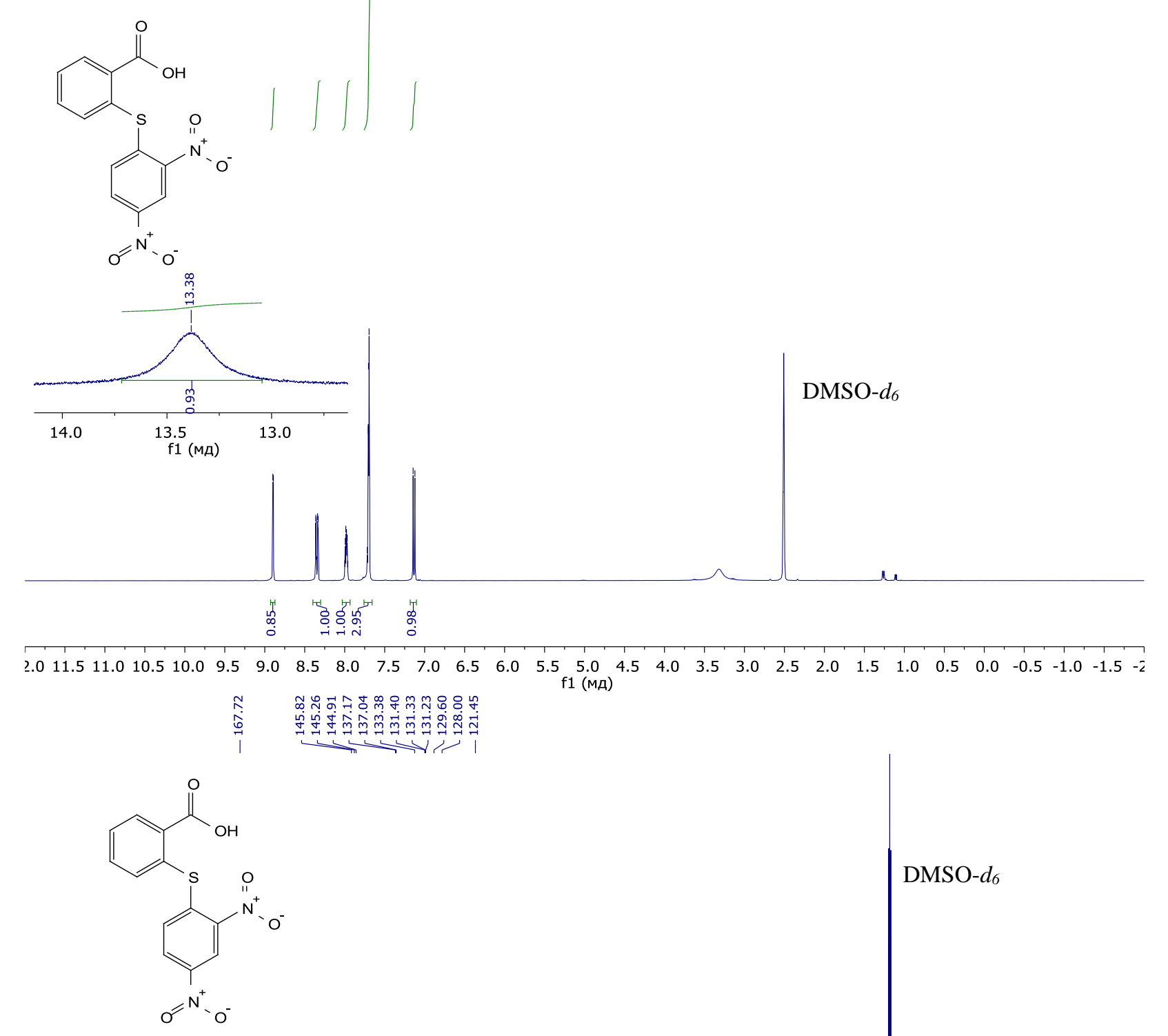

DMSO- $d_{6}$
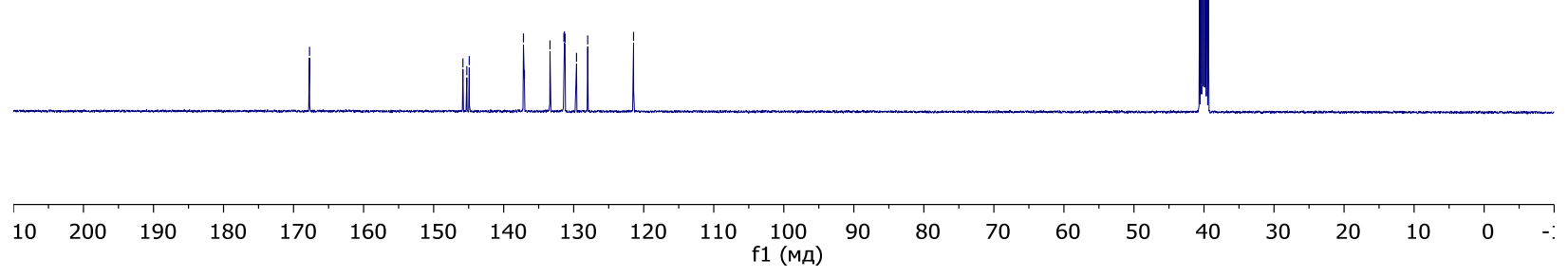

S20 
${ }^{1} \mathrm{H}\left(400 \mathrm{MHz}\right.$; in DMSO- $\left.d_{6}\right)$ and ${ }^{13} \mathrm{C}\left\{{ }^{1} \mathrm{H}\right\}\left(101 \mathrm{MHz}\right.$; in DMSO- $\left.d_{6}\right)$ NMR spectra of compound $8 \mathrm{~b}$

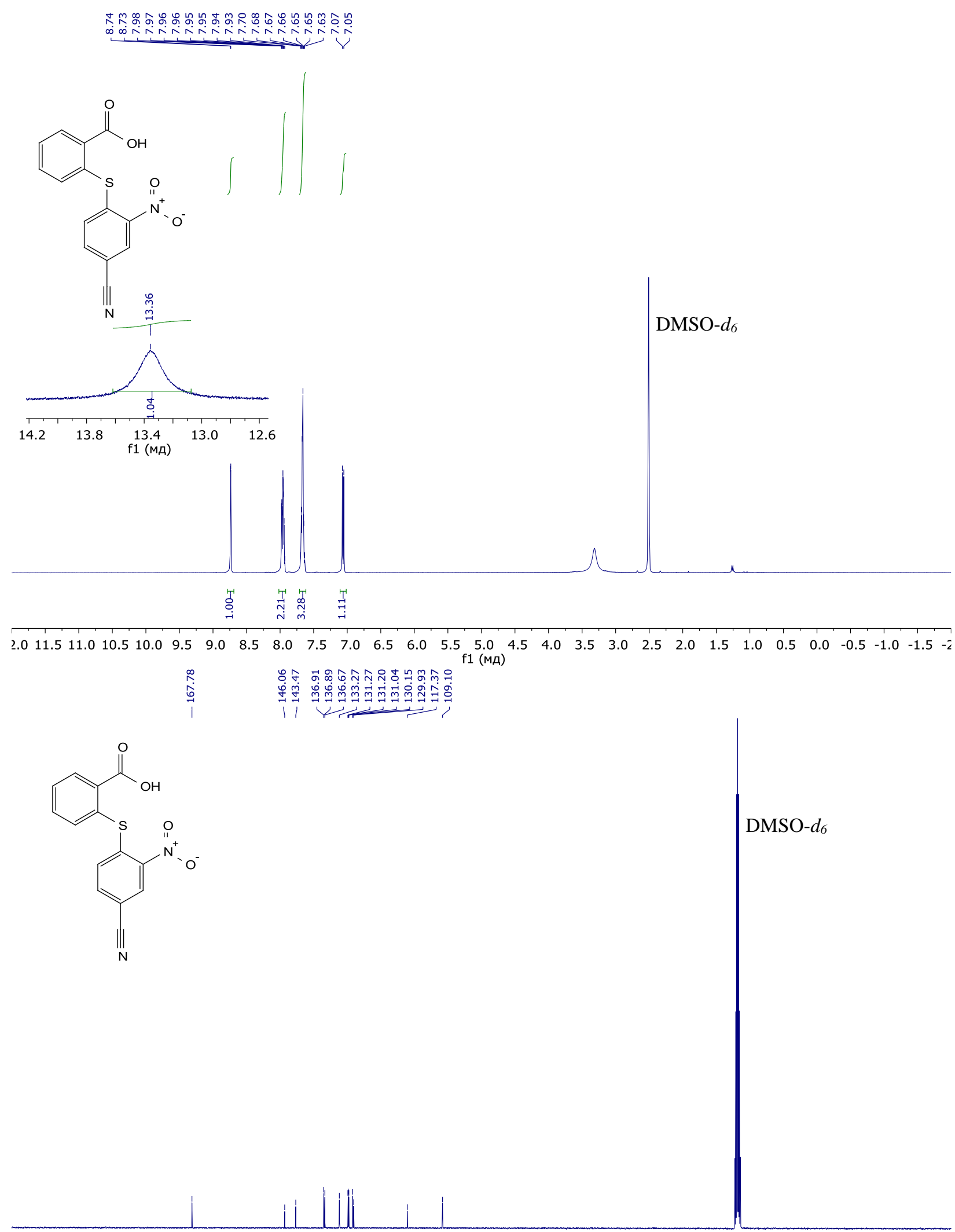

\begin{tabular}{lllllllllllllllllllllll}
\hline 10 & 200 & 190 & 180 & 170 & 160 & 150 & 140 & 130 & 120 & 110 & $\begin{array}{c}100 \\
\mathrm{f} 1(\mathrm{MA})\end{array}$ & 90 & 80 & 70 & 60 & 50 & 40 & 30 & 20 & 10 & 0 & -
\end{tabular} (Mम) 
${ }^{1} \mathrm{H}\left(400 \mathrm{MHz}\right.$; in DMSO- $\left.d_{6}\right)$ and ${ }^{13} \mathrm{C}\left\{{ }^{1} \mathrm{H}\right\}\left(101 \mathrm{MHz}\right.$; in DMSO- $\left.d_{6}\right)$ NMR spectra of compound Bc

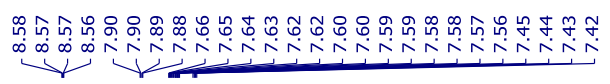
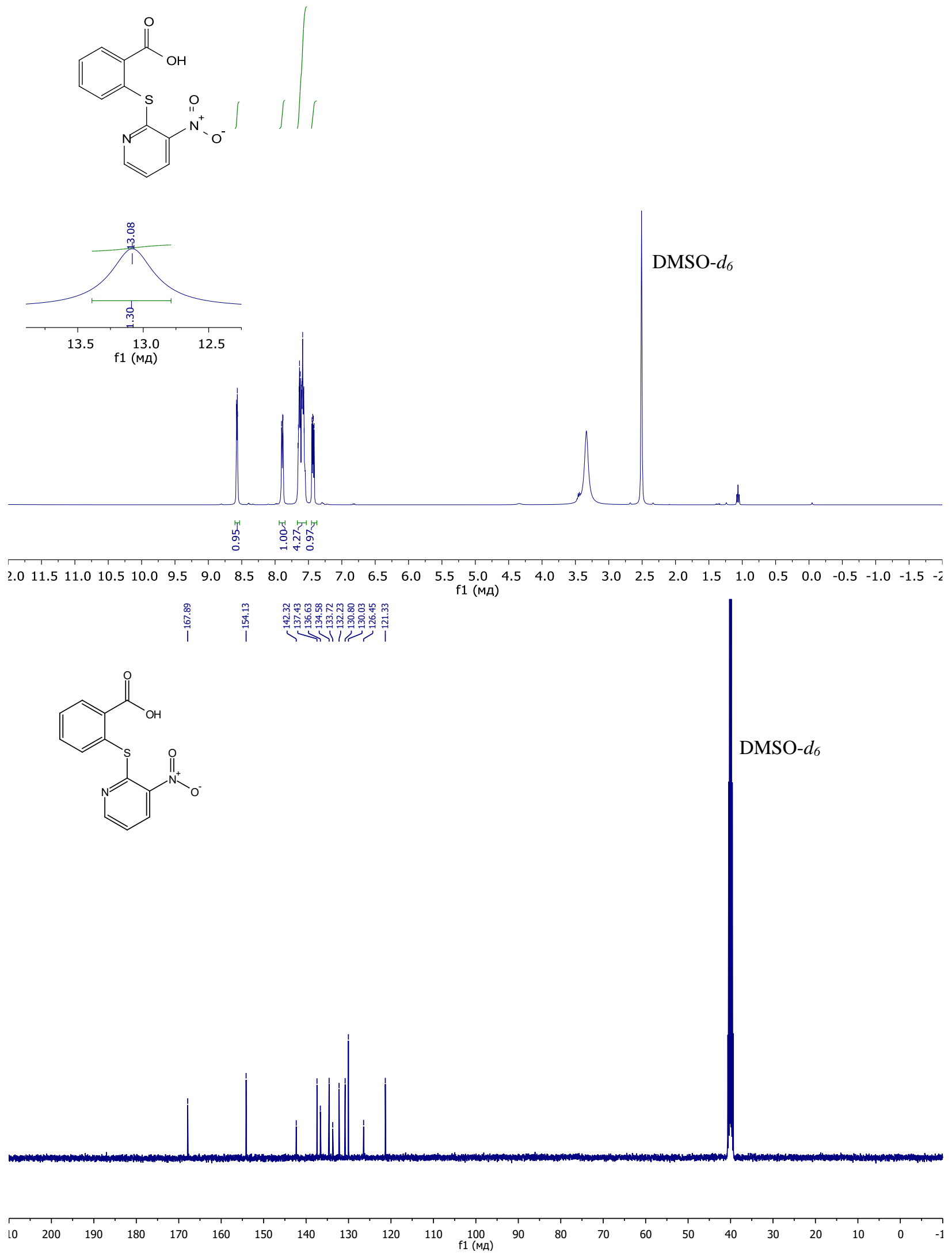

S22 
${ }^{1} \mathrm{H}\left(400 \mathrm{MHz}\right.$; in DMSO- $\left.d_{6}\right)$ and ${ }^{13} \mathrm{C}\left\{{ }^{1} \mathrm{H}\right\}\left(101 \mathrm{MHz}\right.$; in DMSO- $\left.d_{6}\right)$ NMR spectra of compound Bd

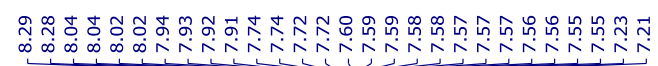
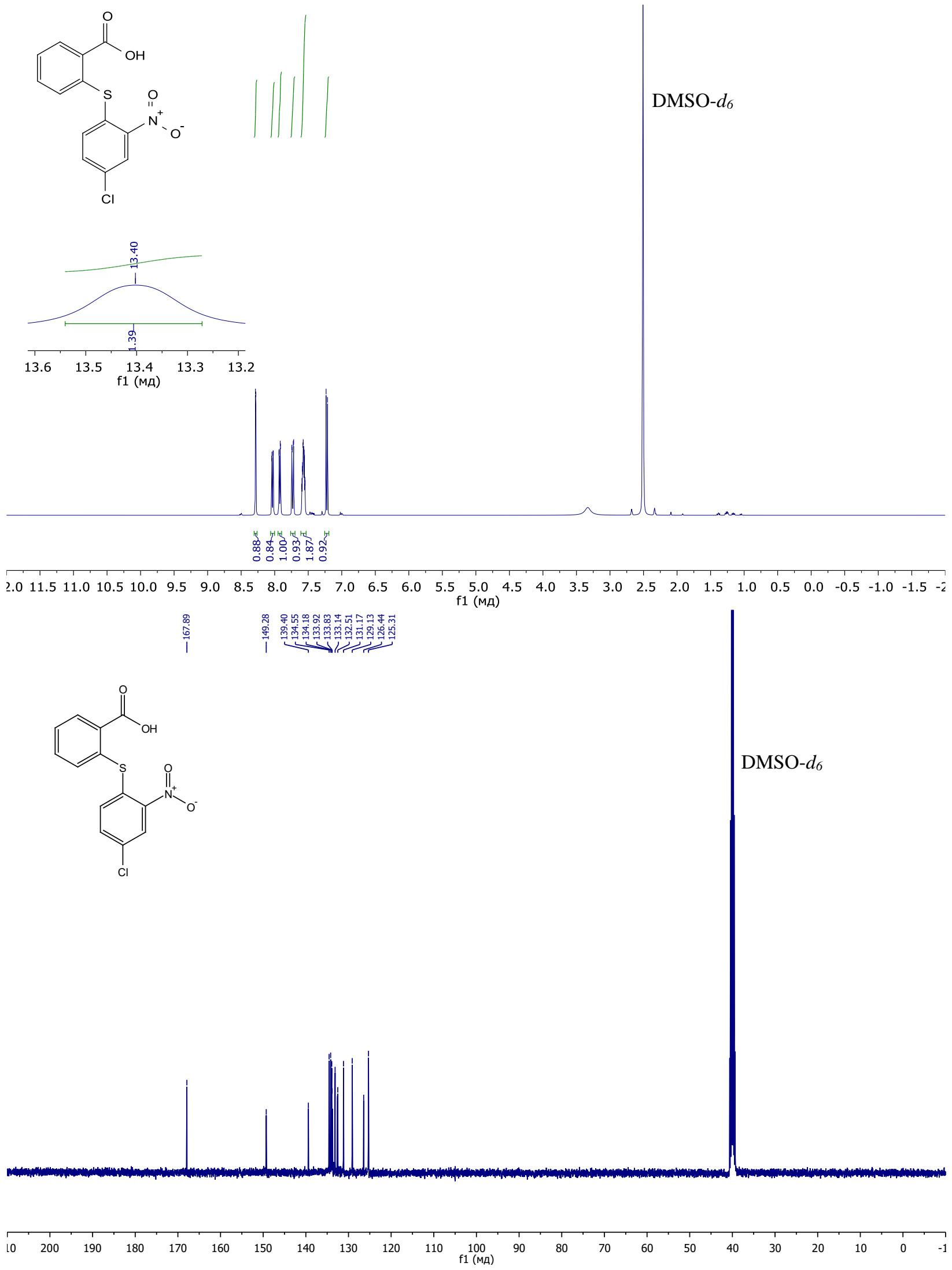

S23 
${ }^{1} \mathrm{H}\left(400 \mathrm{MHz}\right.$; in DMSO- $\left.d_{6}\right)$ and ${ }^{13} \mathrm{C}\left\{{ }^{1} \mathrm{H}\right\}\left(101 \mathrm{MHz}\right.$; in DMSO- $\left.d_{6}\right)$ NMR spectra of compound Be

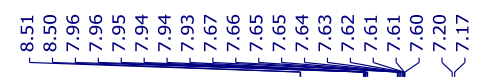
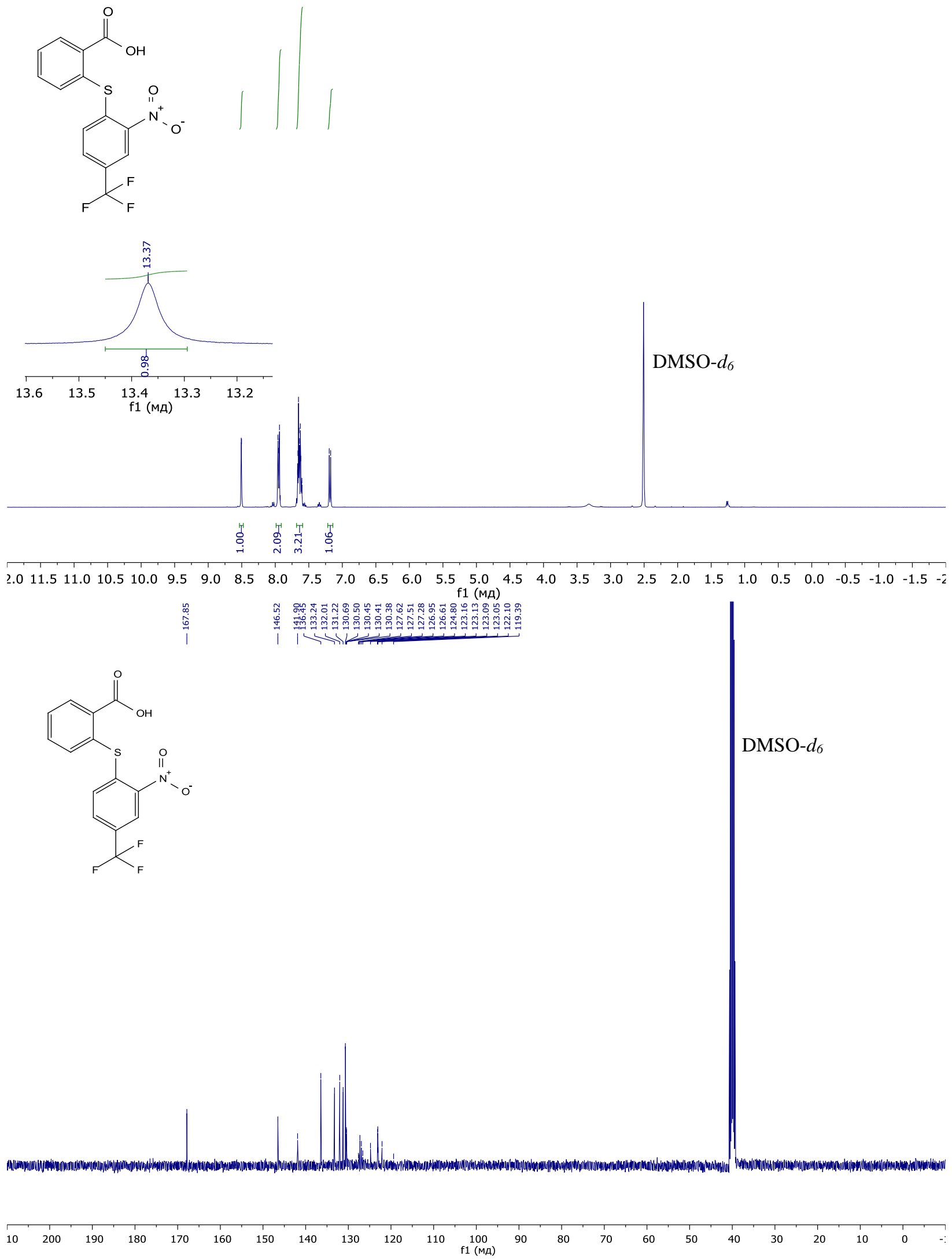

S24 
${ }^{1} \mathrm{H}\left(400 \mathrm{MHz}\right.$; in DMSO- $\left.d_{6}\right)$ and ${ }^{13} \mathrm{C}\left\{{ }^{1} \mathrm{H}\right\}\left(101 \mathrm{MHz}\right.$; in DMSO- $\left.d_{6}\right)$ NMR spectra of compound 8f m̄<smiles>N#Cc1ccc([N+](=O)[O-])c(Sc2ccccc2C(=O)O)c1</smiles>

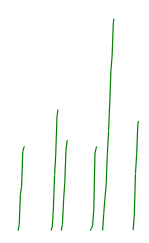

DMSO- $d_{6}$
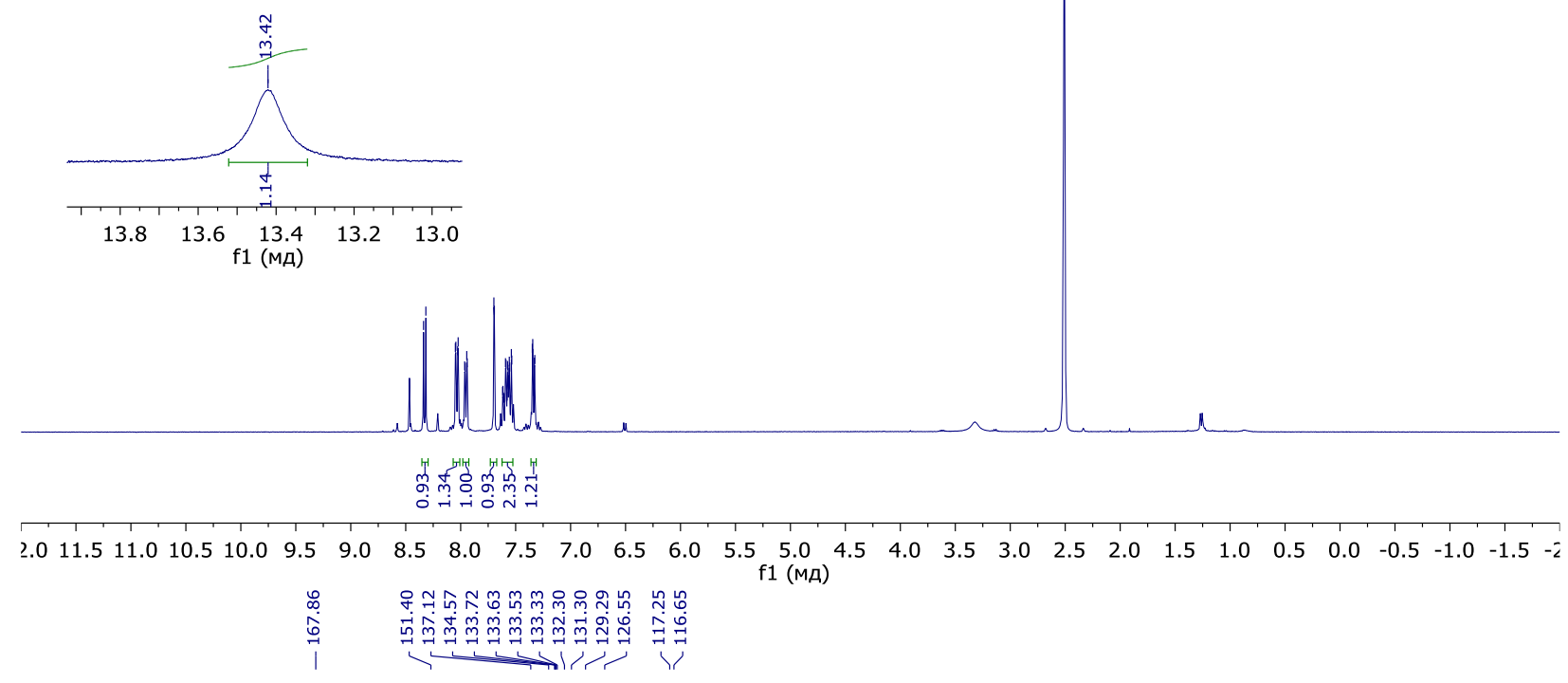<smiles>N#Cc1ccc([N+](=O)[O-])c(Sc2ccccc2C(=O)O)c1</smiles>
DMSO- $d_{6}$ 
${ }^{1} \mathrm{H}\left(400 \mathrm{MHz}\right.$; in DMSO- $\left.d_{6}\right)$ and ${ }^{13} \mathrm{C}\left\{{ }^{1} \mathrm{H}\right\}\left(101 \mathrm{MHz}\right.$; in DMSO- $\left.d_{6}\right)$ NMR spectra of compound $8 \mathrm{~g}$

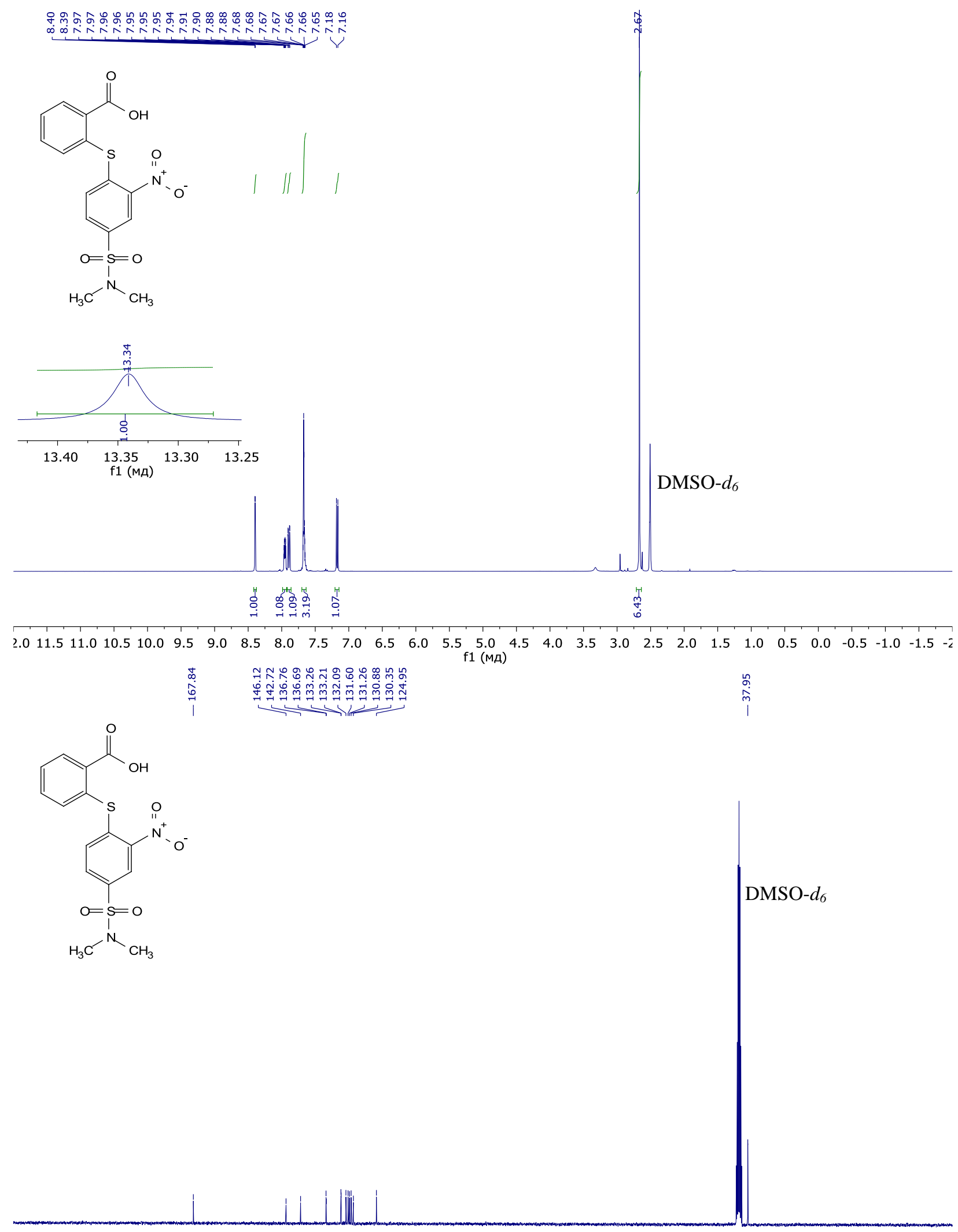

\begin{tabular}{lllllllllllllllllllllll}
\hline 10 & 200 & 190 & 180 & 170 & 160 & 150 & 140 & 130 & 120 & 110 & $\begin{array}{c}100 \\
\mathrm{f} 1 \text { (Mд) }\end{array}$ & 90 & 80 & 70 & 60 & 50 & 40 & 30 & 20 & 10 & 0 &.-
\end{tabular} 
${ }^{1} \mathrm{H}\left(400 \mathrm{MHz}\right.$; in DMSO- $\left.d_{6}\right)$ and ${ }^{13} \mathrm{C}\left\{{ }^{1} \mathrm{H}\right\}\left(101 \mathrm{MHz}\right.$; in DMSO- $\left.d_{6}\right)$ NMR spectra of compound $8 \mathrm{~h}$
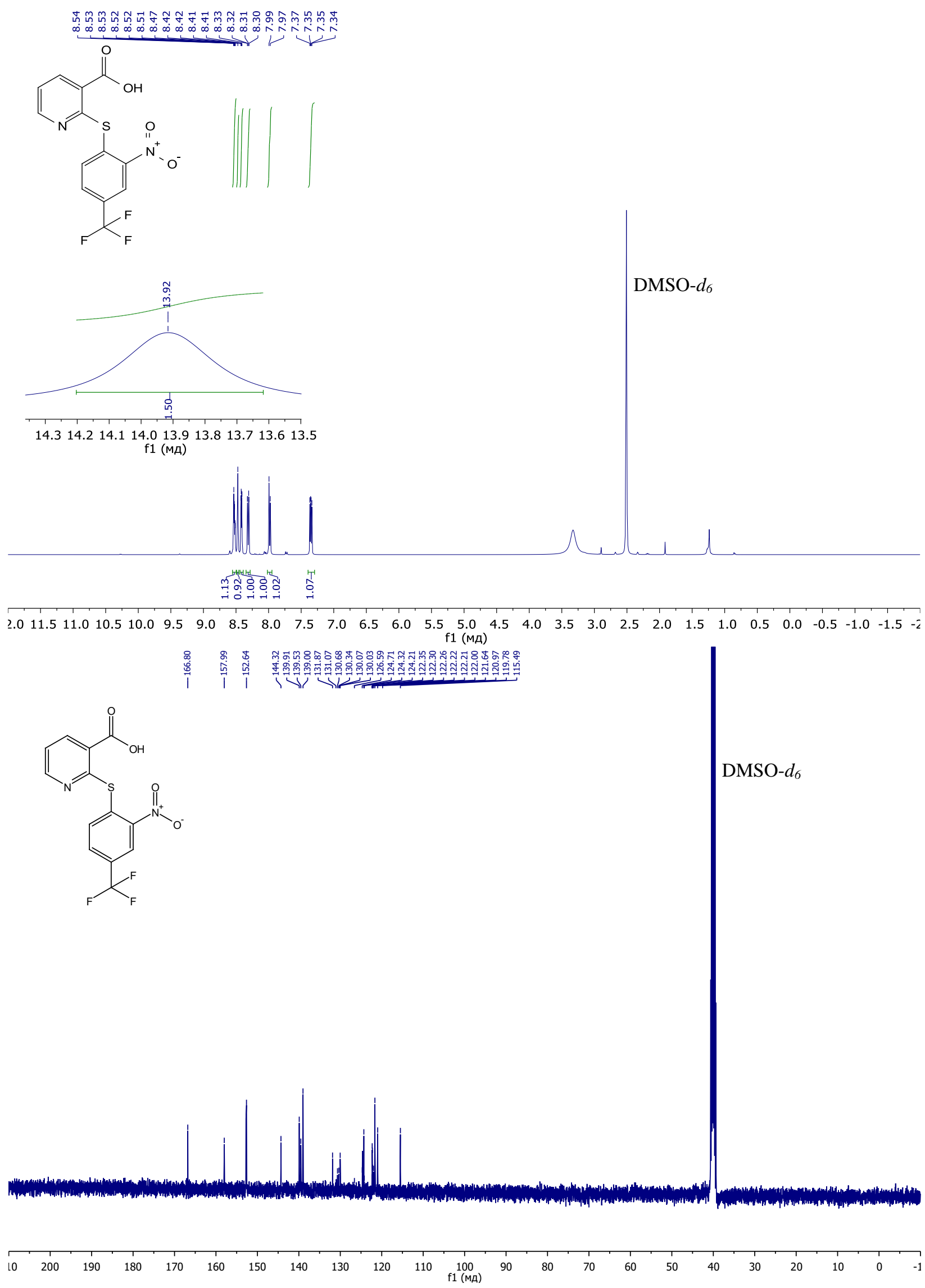

S27 
${ }^{1} \mathrm{H}\left(400 \mathrm{MHz}\right.$; in $\left.\mathrm{CDCl}_{3}\right)$ and ${ }^{13} \mathrm{C}\left\{{ }^{1} \mathrm{H}\right\}\left(101 \mathrm{MHz}\right.$; in $\left.\mathrm{CDCl}_{3}\right) \mathrm{NMR}$ spectra of compound qa

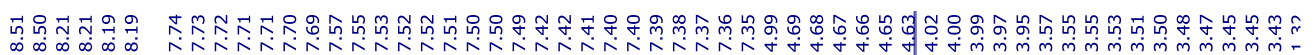
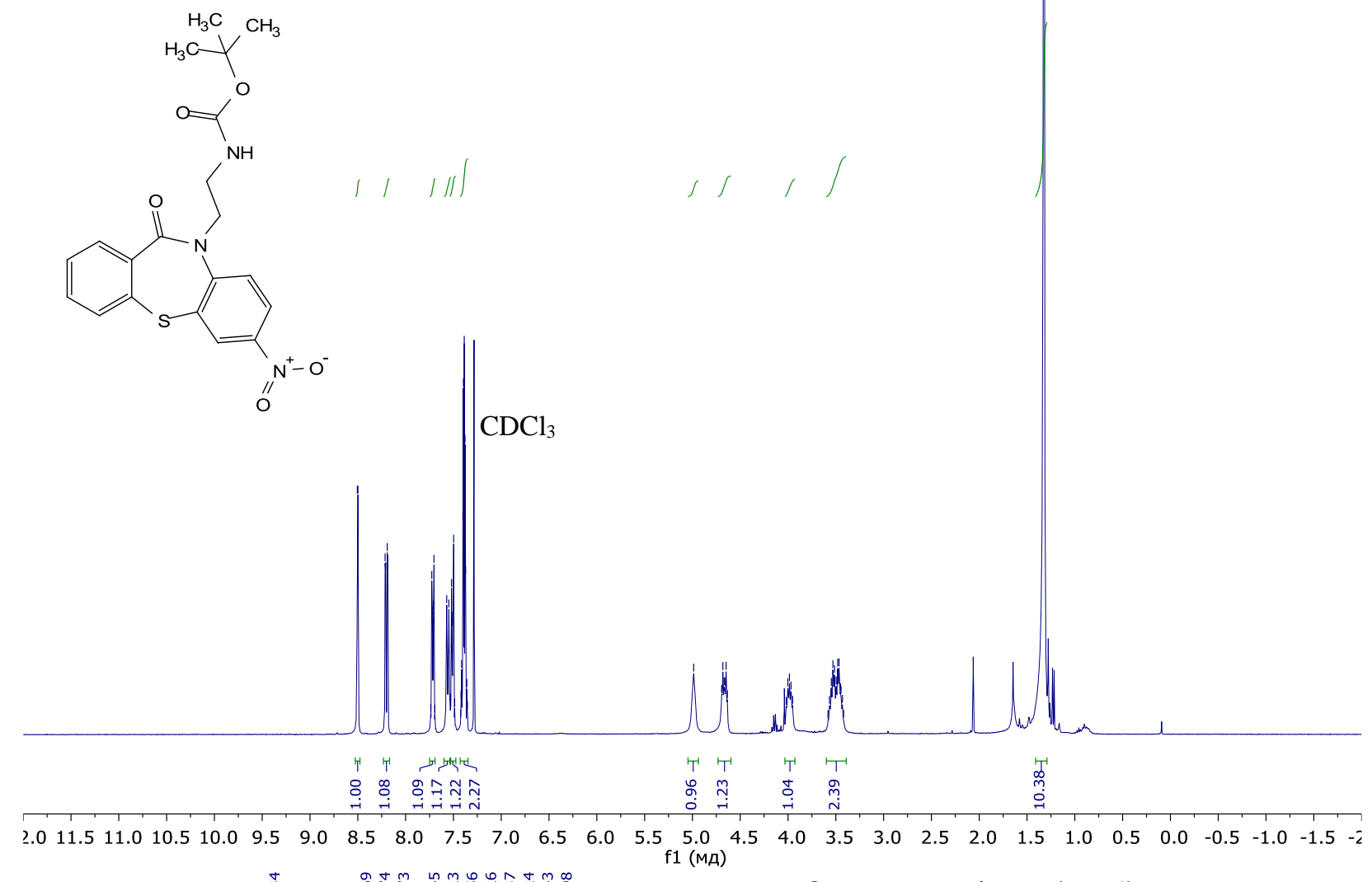

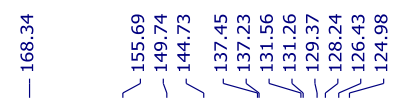
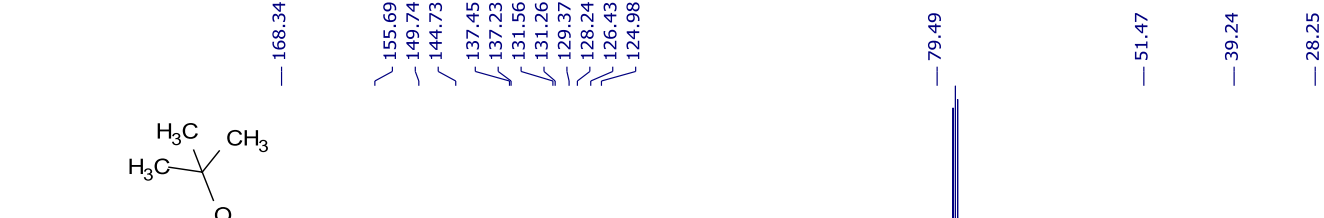<smiles>NC(=O)[O-]</smiles>

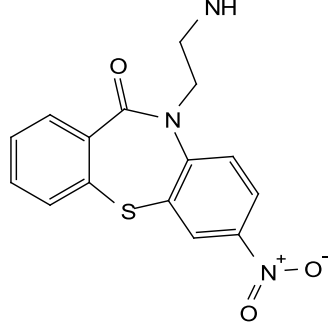

$\mathrm{CDCl}_{3}$
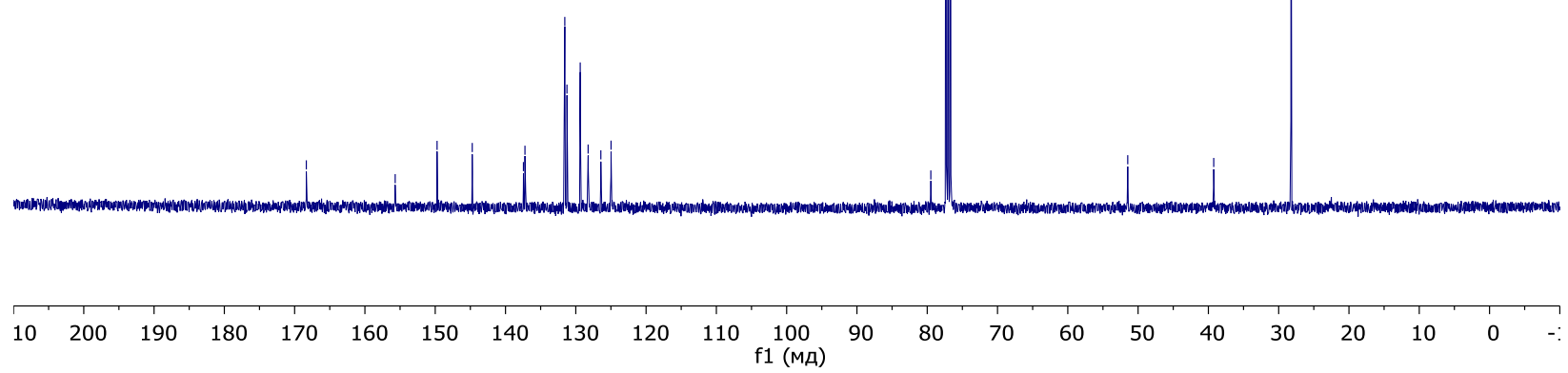

S28 
${ }^{1} \mathrm{H}\left(400 \mathrm{MHz}\right.$; in $\left.\mathrm{CDCl}_{3}\right)$ and ${ }^{13} \mathrm{C}\left\{{ }^{1} \mathrm{H}\right\}\left(101 \mathrm{MHz}\right.$; in $\left.\mathrm{CDCl}_{3}\right)$ NMR spectra of compound 9b

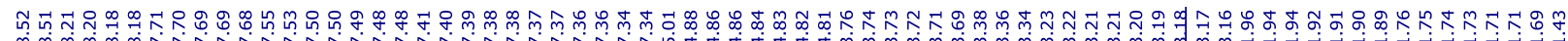<smiles>CC(C)(C)OC(=O)NCCCN1C(=O)c2ccccc2Sc2cc([N+](=O)[O-])ccc21</smiles>
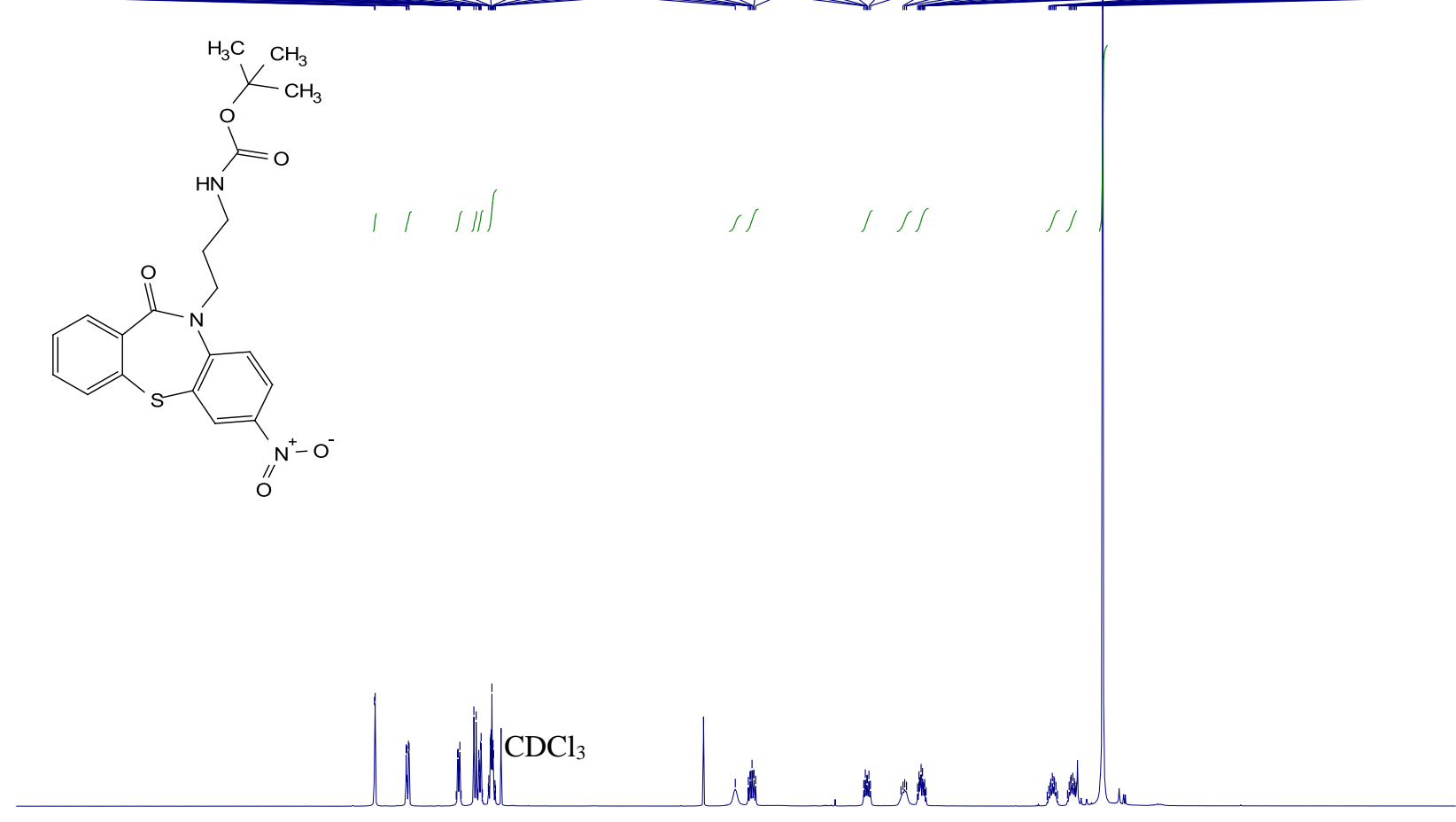

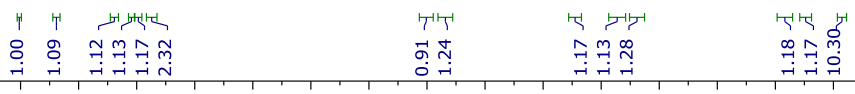

$\begin{array}{lllllllllllllllllllllllllllll}2.0 & 11.5 & 11.0 & 10.5 & 10.0 & 9.5 & 9.0 & 8.5 & 8.0 & 7.5 & 7.0 & 6.5 & 6.0 & 5.5 & 5.0 & 4.5 & 4.0 & 3.5 & 3.0 & 2.5 & 2.0 & 1.5 & 1.0 & 0.5 & 0.0 & -0.5 & -1.0 & -1.5 & -z\end{array}$

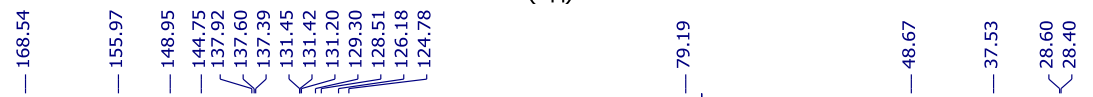<smiles>CC(C)(C)OC(=O)NCCCN1C(=O)c2ccccc2Sc2cc([N+](=O)[O-])ccc21</smiles>

$\mathrm{CDCl}_{3}$

$\begin{array}{lllllllllllllllllllllll}10 & 200 & 190 & 180 & 170 & 160 & 150 & 140 & 130 & 120 & 110 & 100 & 90 & 80 & 70 & 60 & 50 & 40 & 30 & 20 & 10 & 0 & -\end{array}$ 
${ }^{1} \mathrm{H}\left(400 \mathrm{MHz}\right.$; in $\left.\mathrm{CDCl}_{3}\right)$ and ${ }^{13} \mathrm{C}\left\{{ }^{1} \mathrm{H}\right\}\left(101 \mathrm{MHz}\right.$; in $\left.\mathrm{CDCl}_{3}\right)$ NMR spectra of compound 9c

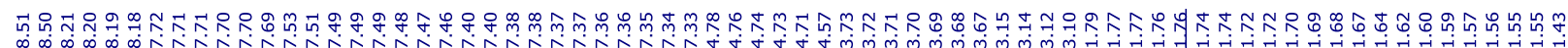<smiles>CC(C)(C)OC(=O)NCCCCN1C(=O)c2ccccc2Sc2cc([N+](=O)[O-])ccc21</smiles>

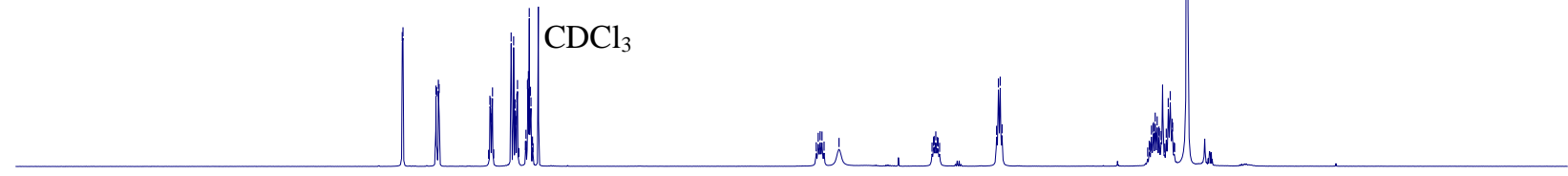

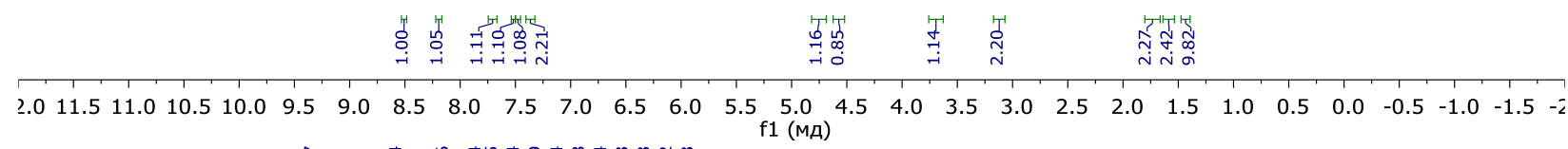

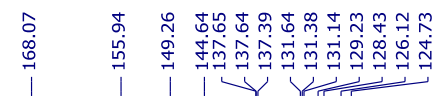<smiles>CC(C)(C)OC(=O)NCCCCN1C(=O)c2ccccc2Sc2cc([N+](=O)[O-])ccc21</smiles>

$\mathrm{CDCl}_{3}$

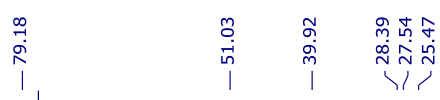

10200

$\begin{array}{llllllll}00 & 190 & 180 & 170 & 160 & 150 & 140 & 130\end{array}$

$\begin{array}{lll}120 & 110 & 100\end{array}$ f1 (Мд) 
${ }^{1} \mathrm{H}\left(400 \mathrm{MHz}\right.$; in $\left.\mathrm{CDCl}_{3}\right)$ and ${ }^{13} \mathrm{C}\left\{{ }^{1} \mathrm{H}\right\}\left(101 \mathrm{MHz}\right.$; in $\left.\mathrm{CDCl}_{3}\right)$ NMR spectra of compound 9d

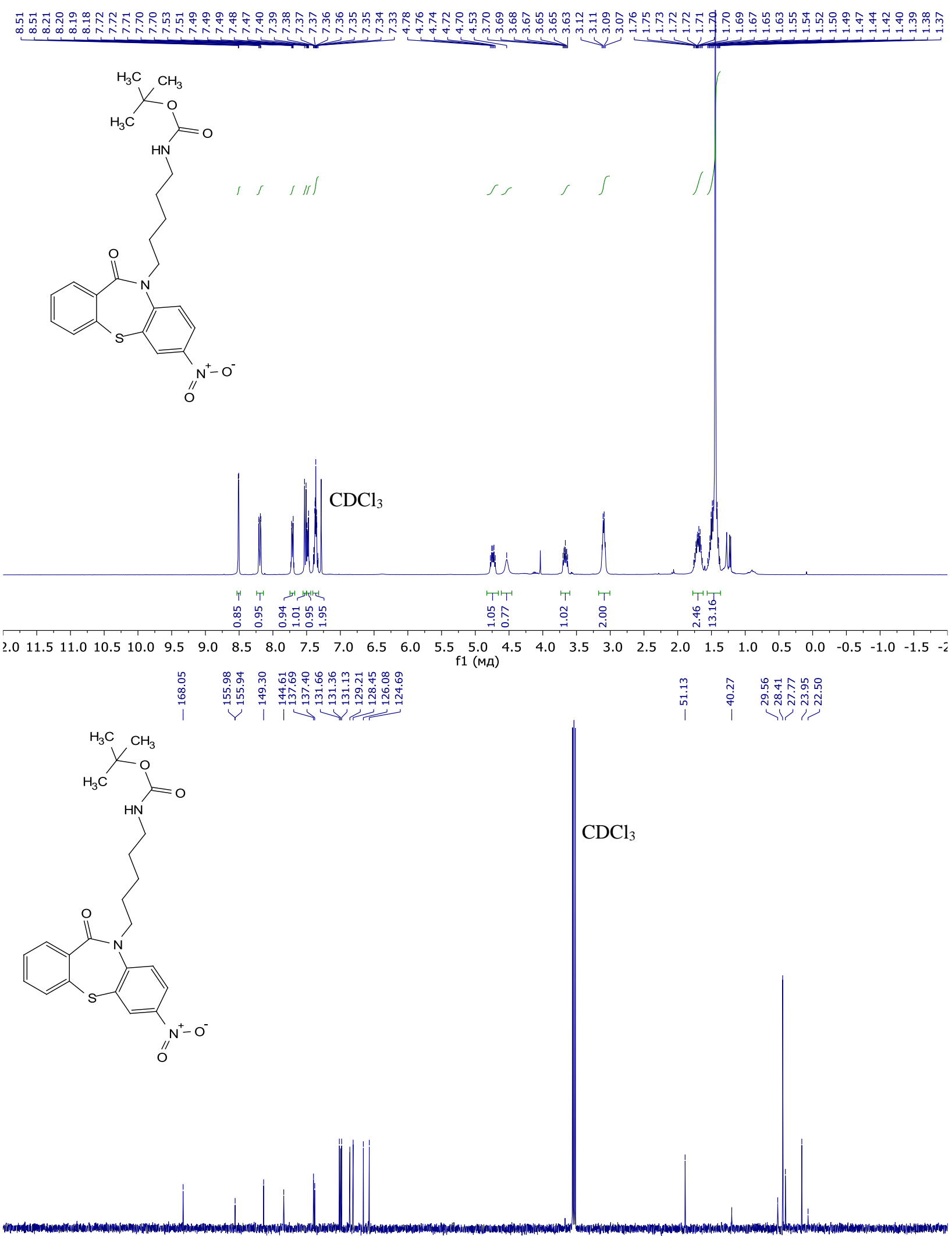

$\begin{array}{llllllllllllllllllllllllllll}10 & 200 & 190 & 180 & 170 & 160 & 150 & 140 & 130 & 120 & 110 & 100 & 90 & 80 & 70 & 60 & 50 & 40 & 30 & 20 & 10 & 0 & -\end{array}$ 
${ }^{1} \mathrm{H}\left(400 \mathrm{MHz}\right.$; in $\left.\mathrm{CDCl}_{3}\right)$ and ${ }^{13} \mathrm{C}\left\{{ }^{1} \mathrm{H}\right\}\left(101 \mathrm{MHz}\right.$; in $\left.\mathrm{CDCl}_{3}\right)$ NMR spectra of compound Me

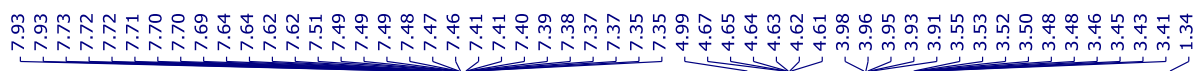
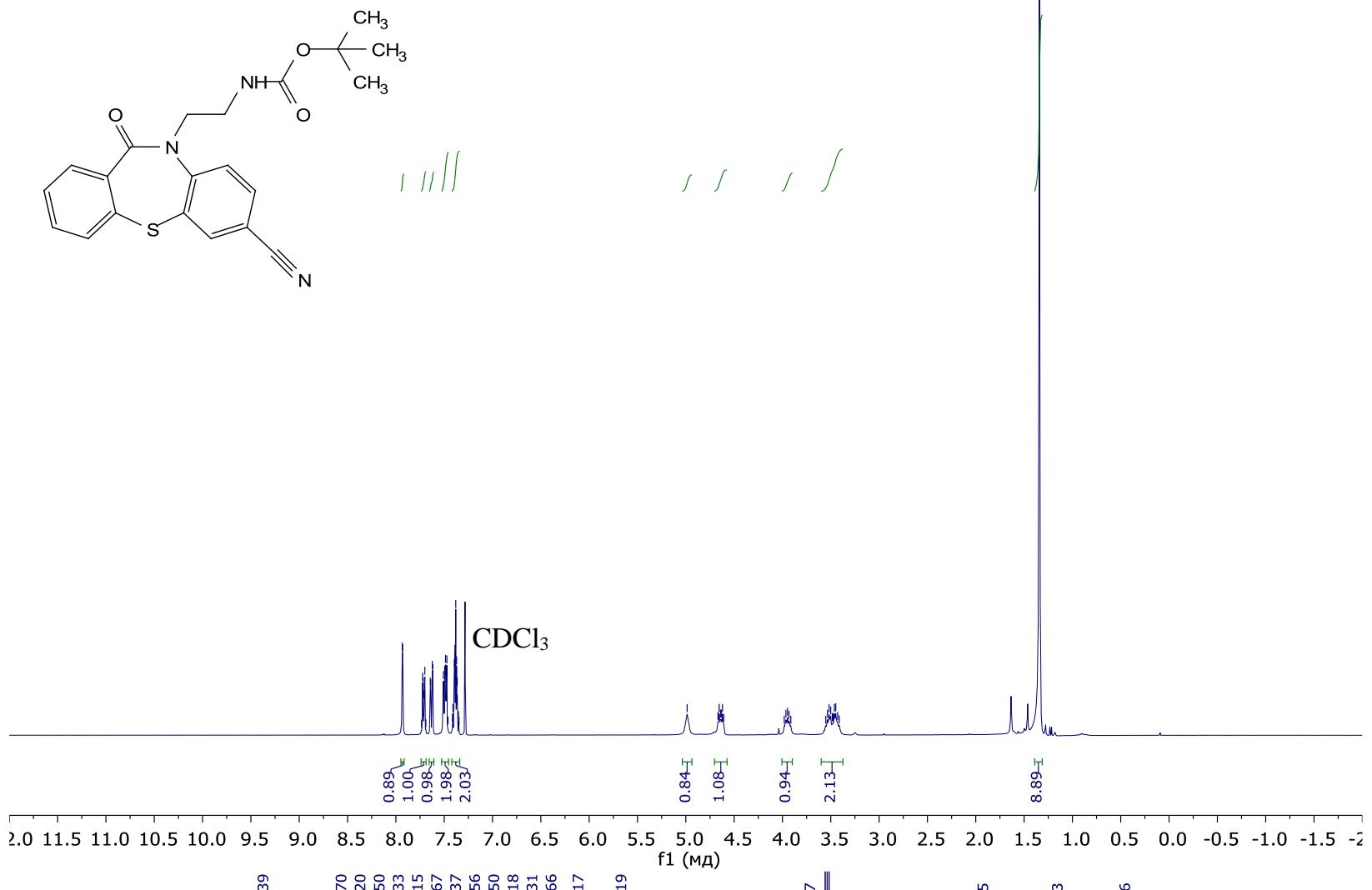

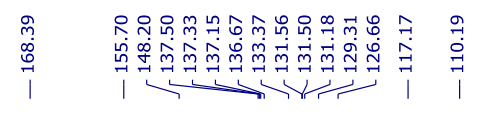
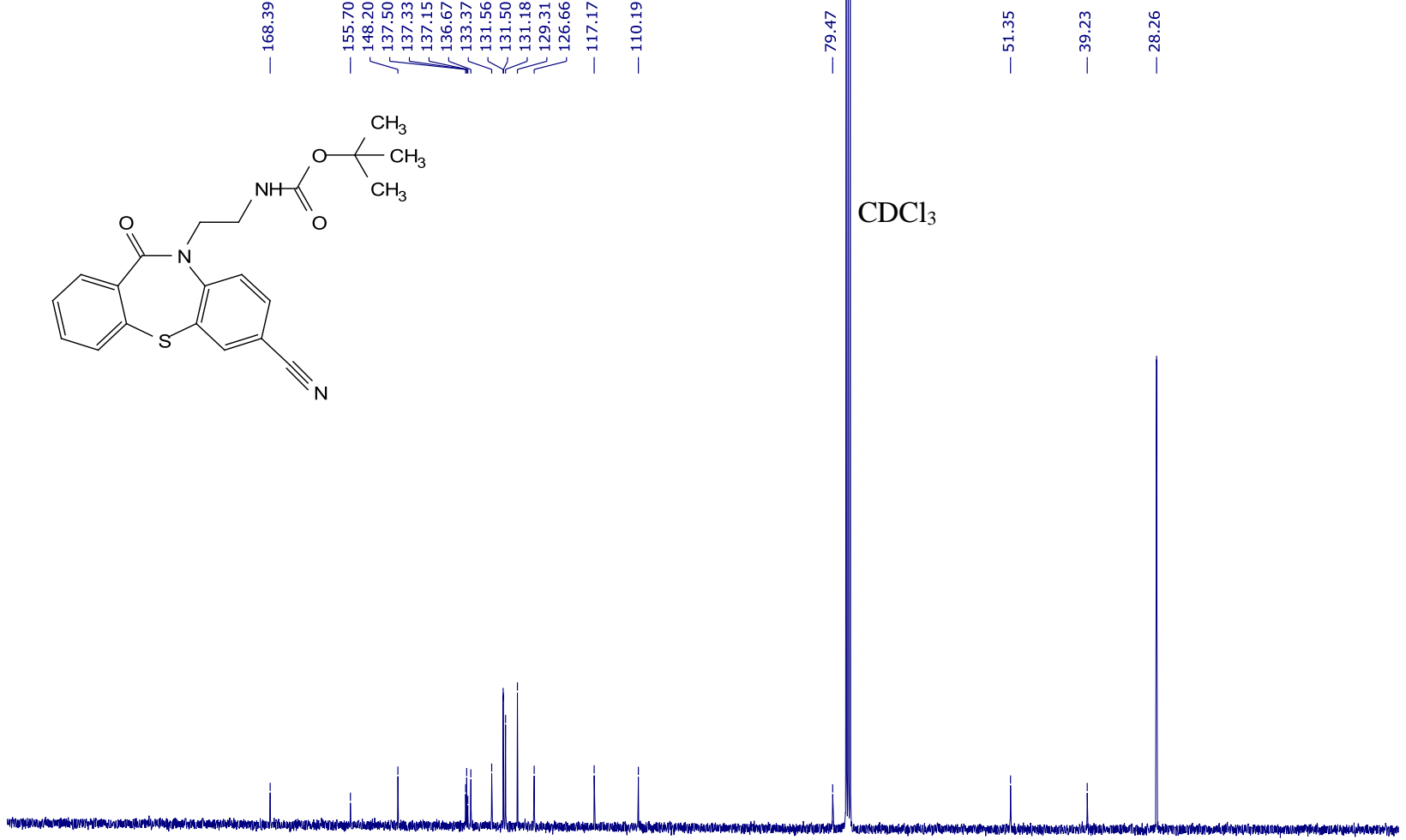

$\mathrm{CDCl}_{3}$

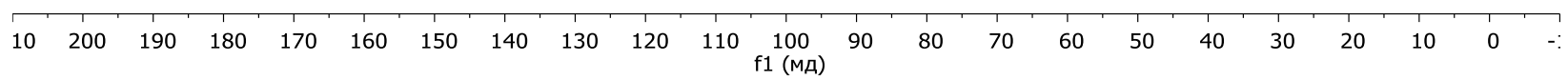

S32 


\section{${ }^{1} \mathrm{H}\left(400 \mathrm{MHz}\right.$; in $\left.\mathrm{CDCl}_{3}\right)$ and ${ }^{13} \mathrm{C}\left\{{ }^{1} \mathrm{H}\right\}\left(101 \mathrm{MHz}\right.$; in $\left.\mathrm{CDCl}_{3}\right) \mathrm{NMR}$ spectra of compound $9 \mathrm{f}$}

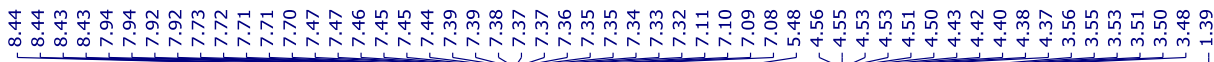<smiles>CC(C)(C)OC(=O)NCCN1C(=O)c2ccccc2Sc2cccnc21</smiles>
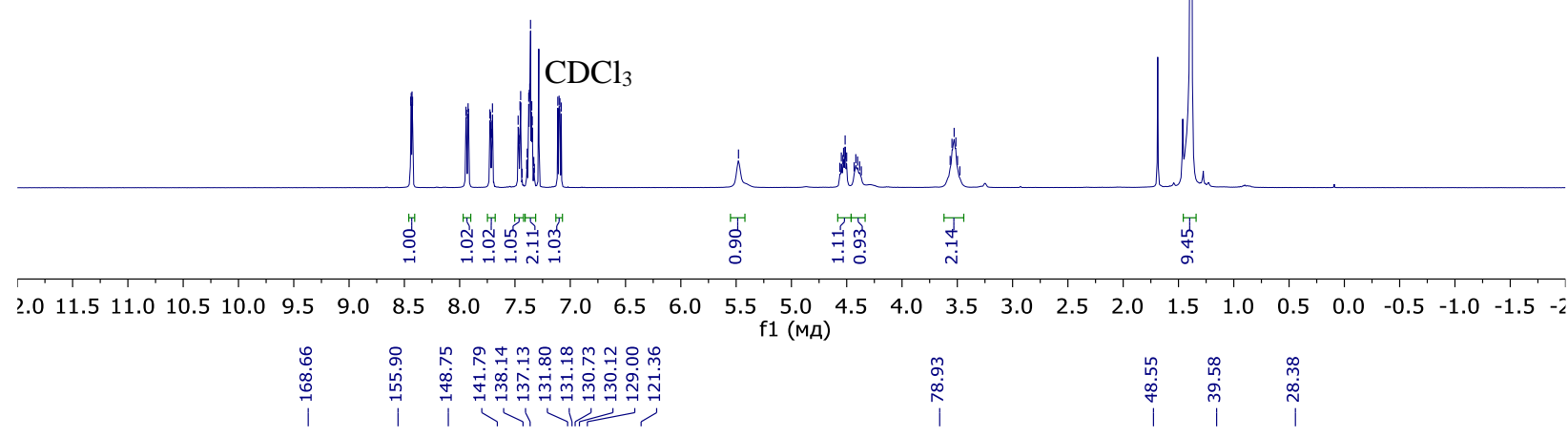<smiles>CC(C)(C)OC(=O)NCCN1C(=O)c2ccccc2Sc2cccnc21</smiles>

$\mathrm{CDCl}_{3}$

$\begin{array}{llllllllllll}10 & 200 & 190 & 180 & 170 & 160 & 150 & 140 & 130 & 120 & 110 & \begin{array}{c}100 \\ \mathrm{f} 1(\mathrm{MP})\end{array}\end{array}$ 
${ }^{1} \mathrm{H}\left(400 \mathrm{MHz}\right.$; in $\left.\mathrm{CDCl}_{3}\right)$ and ${ }^{13} \mathrm{C}\left\{{ }^{1} \mathrm{H}\right\}\left(101 \mathrm{MHz}\right.$; in $\left.\mathrm{CDCl}_{3}\right)$ NMR spectra of compound $9 \mathrm{~g}$

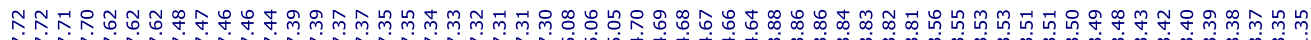

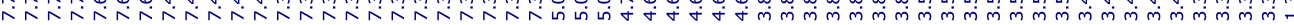<smiles>CC(C)(C)OC(=O)NCCN1C(=O)c2ccccc2Sc2cc(Cl)ccc21</smiles>

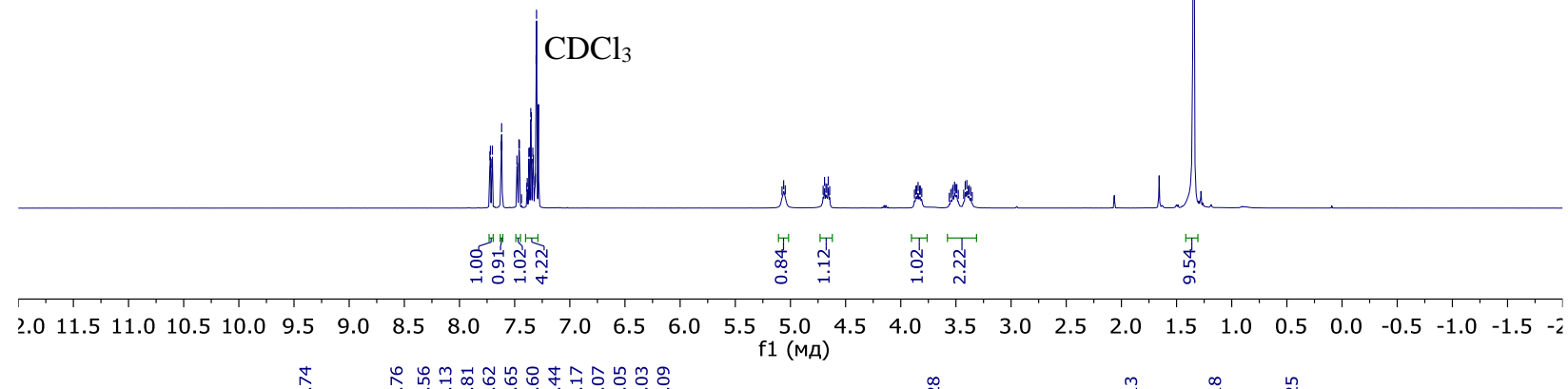

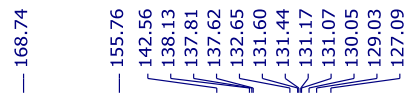<smiles>CC(C)(C)OC(=O)NCCN1C(=O)c2ccccc2Sc2cc(Cl)ccc21</smiles>
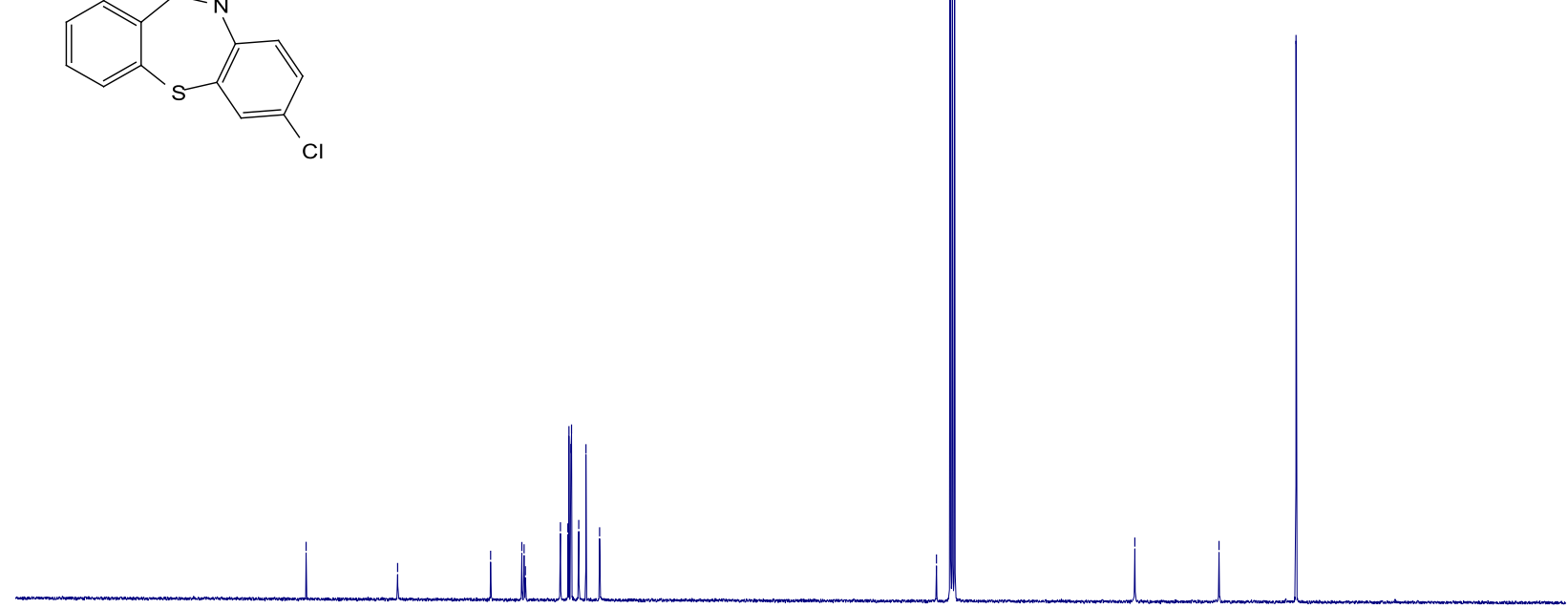
${ }^{1} \mathrm{H}\left(400 \mathrm{MHz}\right.$; in $\left.\mathrm{CDCl}_{3}\right)$ and ${ }^{13} \mathrm{C}\left\{{ }^{1} \mathrm{H}\right\}\left(101 \mathrm{MHz}\right.$; in $\left.\mathrm{CDCl}_{3}\right)$ NMR spectra of compound $9 \mathrm{~h}$

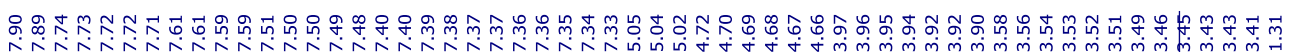

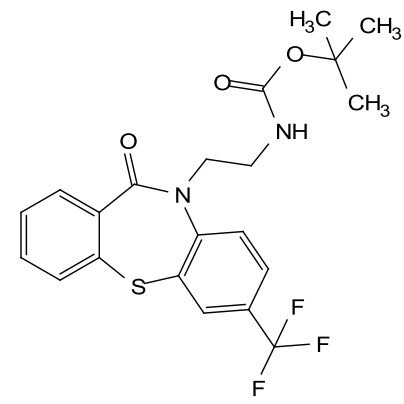

(all
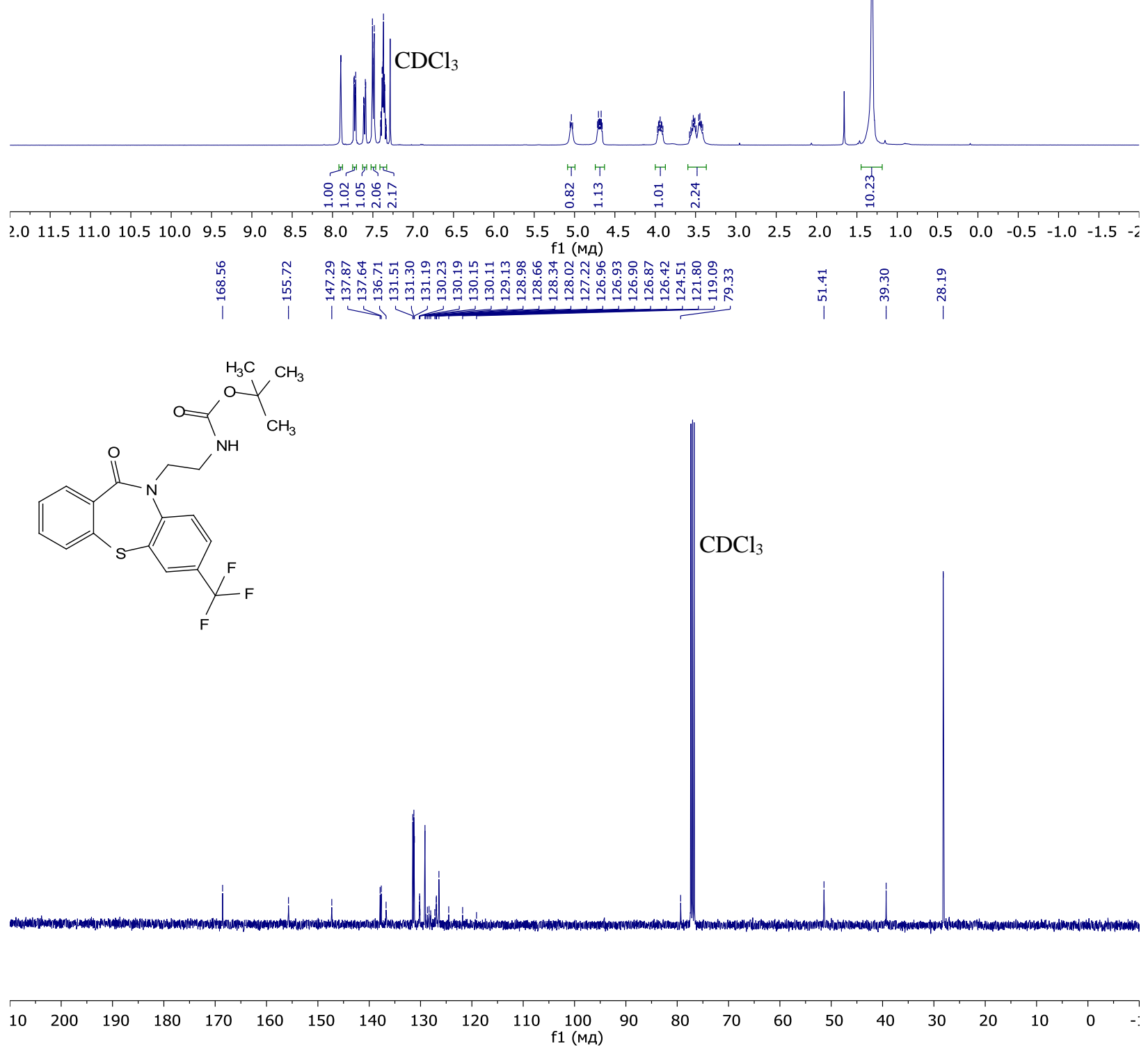

S35 
${ }^{1} \mathrm{H}\left(400 \mathrm{MHz}\right.$; in $\left.\mathrm{CDCl}_{3}\right)$ and ${ }^{13} \mathrm{C}\left\{{ }^{1} \mathrm{H}\right\}\left(101 \mathrm{MHz}\right.$; in $\left.\mathrm{CDCl}_{3}\right)$ NMR spectra of compound $9 \mathrm{i}$

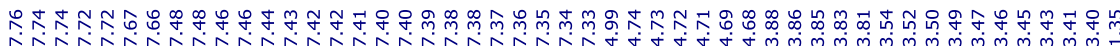

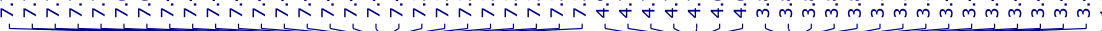
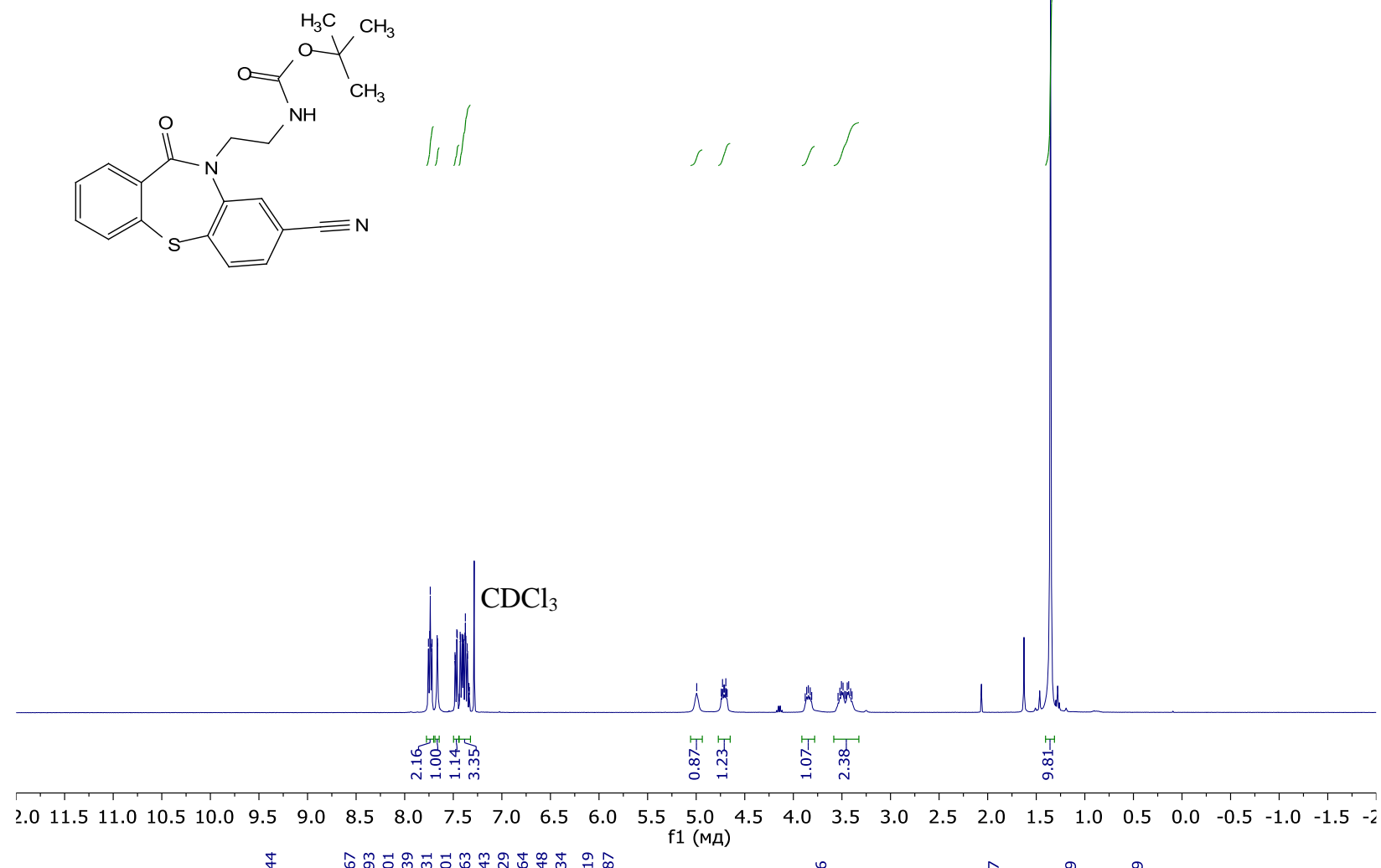

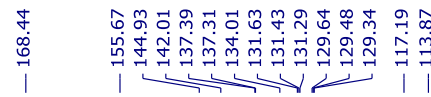

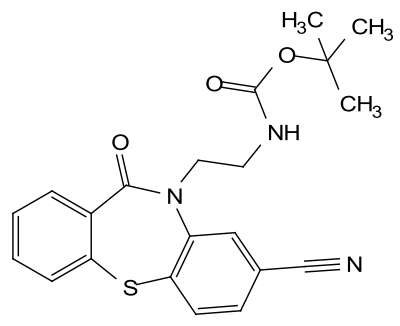

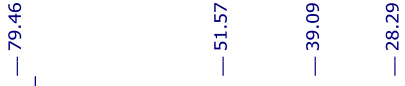

$\mathrm{CDCl}_{3}$

$\begin{array}{llllllllllll}10 & 200 & 190 & 180 & 170 & 160 & 150 & 140 & 130 & 120 & 110 & 100 \\ \mathrm{f} 1(\mathrm{MA})\end{array}$ 
${ }^{1} \mathrm{H}\left(400 \mathrm{MHz}\right.$; in $\left.\mathrm{CDCl}_{3}\right)$ and ${ }^{13} \mathrm{C}\left\{{ }^{1} \mathrm{H}\right\}\left(101 \mathrm{MHz}\right.$; in $\left.\mathrm{CDCl}_{3}\right) \mathrm{NMR}$ spectra of compound $9 \mathrm{j}$

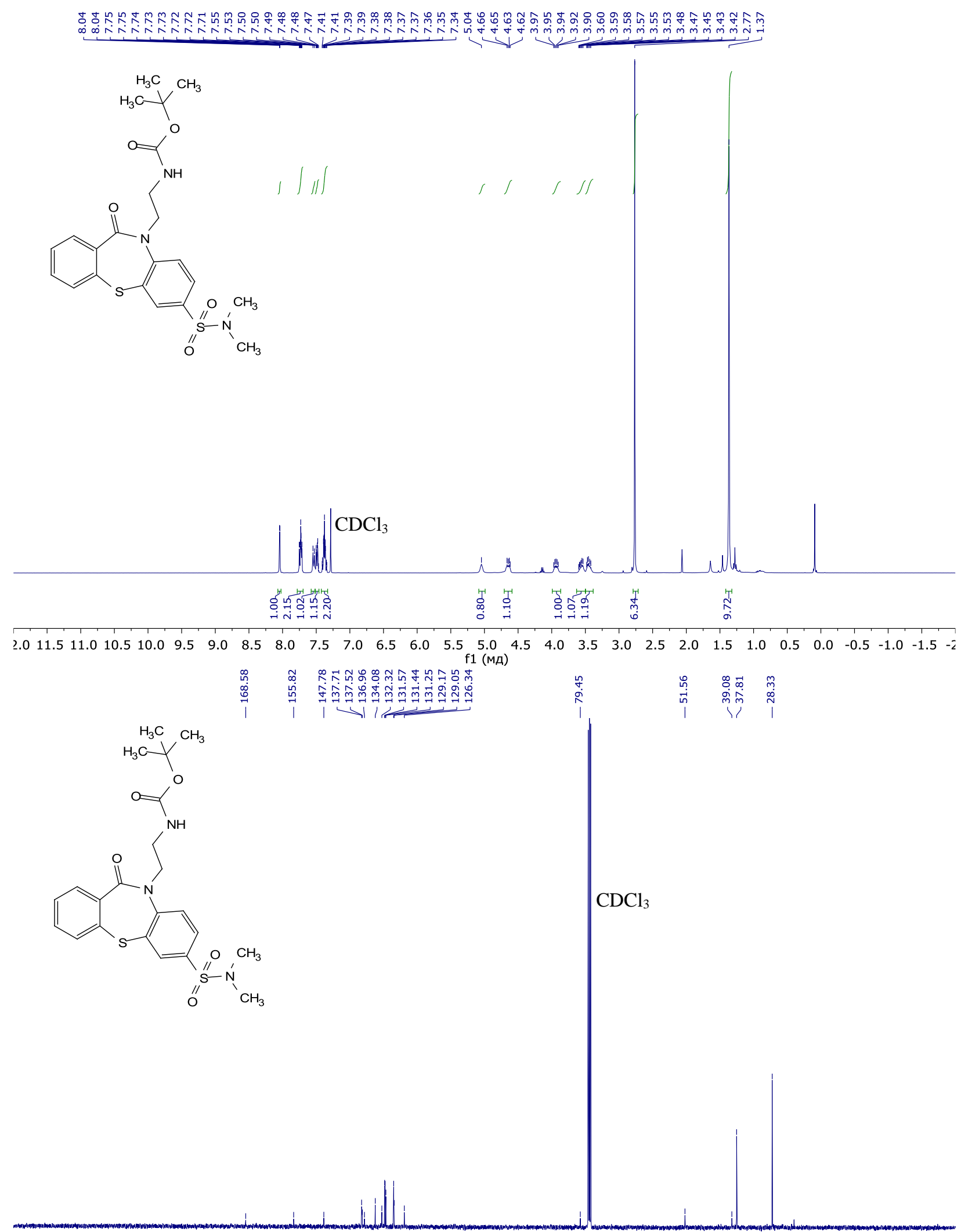

$\begin{array}{llllllllllllllllllllllllllllll}30 & 220 & 210 & 200 & 190 & 180 & 170 & 160 & 150 & 140 & 130 & 120 & 110 & 100 & 90 & 80 & 70 & 60 & 50 & 40 & 30 & 20 & 10 & 0 & -10 & -z\end{array}$ 
${ }^{1} \mathrm{H}\left(400 \mathrm{MHz}\right.$; in $\left.\mathrm{CDCl}_{3}\right)$ and ${ }^{13} \mathrm{C}\left\{{ }^{1} \mathrm{H}\right\}\left(126 \mathrm{MHz}\right.$; in $\left.\mathrm{CDCl}_{3}\right)$ NMR spectra of compound 9k

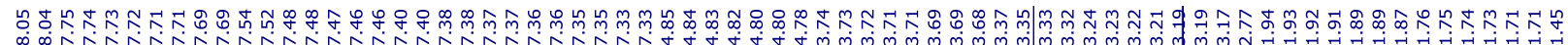<smiles>CN(C)S(=O)(=O)c1ccc2c(c1)Sc1ccccc1C(=O)N2CCCNC(=O)OC(C)(C)C</smiles>

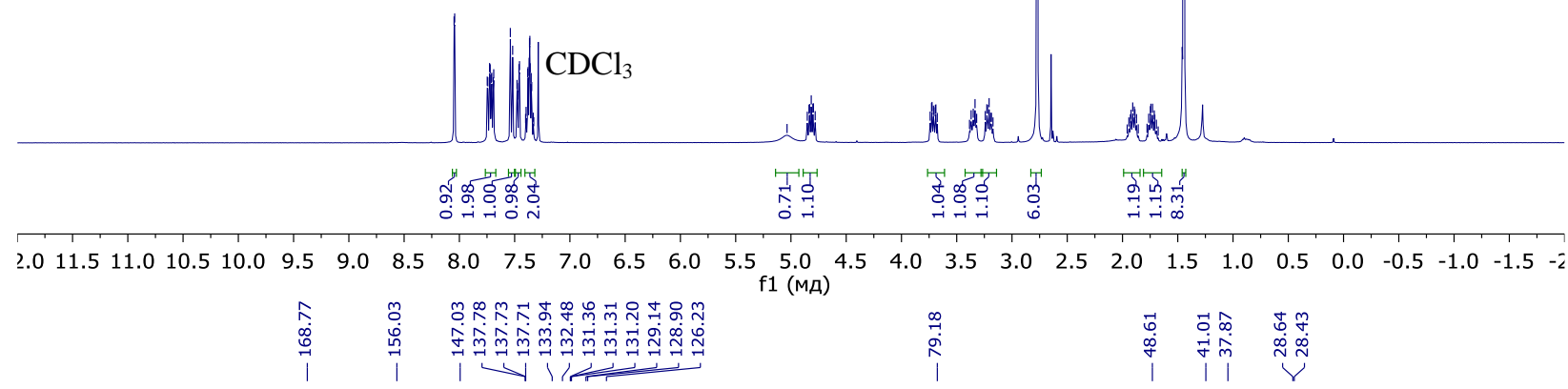<smiles>CN(C)S(=O)(=O)c1ccc2c(c1)Sc1ccccc1C(=O)N2CCCNC(=O)OC(C)(C)C</smiles>

$\mathrm{CDCl}_{3}$

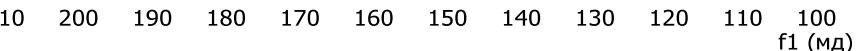


${ }^{1} \mathrm{H}\left(400 \mathrm{MHz}\right.$; in $\left.\mathrm{CDCl}_{3}\right)$ and ${ }^{13} \mathrm{C}\left\{{ }^{1} \mathrm{H}\right\}\left(101 \mathrm{MHz}\right.$; in $\left.\mathrm{CDCl}_{3}\right)$ NMR spectra of compound $9 \mathrm{l}$

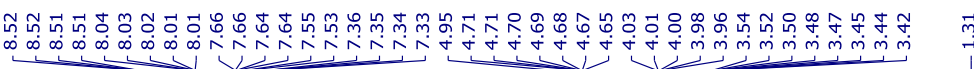<smiles>CC(C)(C)OC(N)=O</smiles><smiles>CNCCN1C(=O)c2cccnc2Sc2cc(C(F)(F)F)ccc21</smiles>

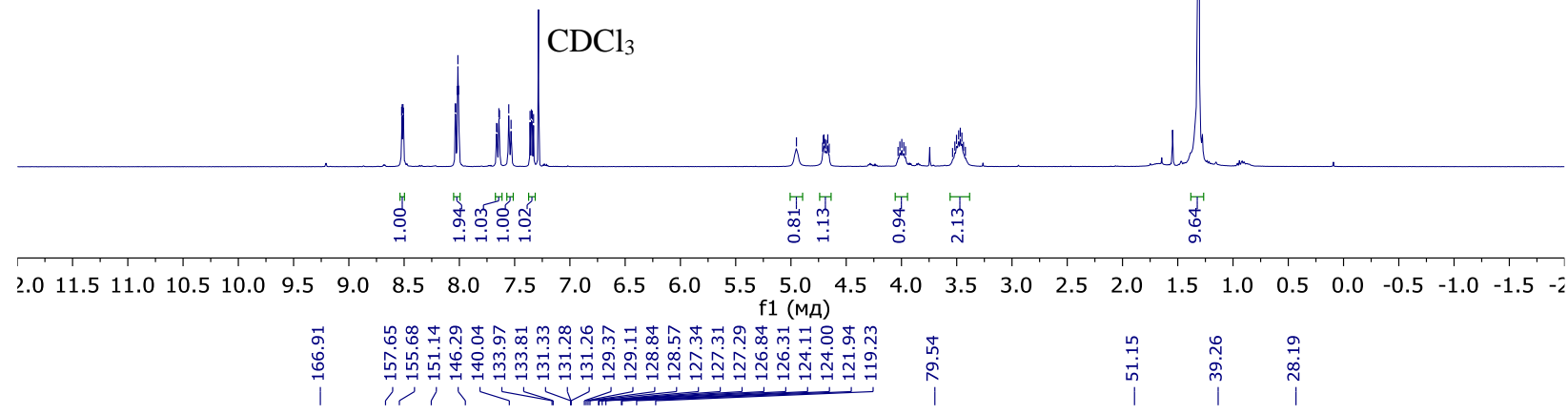<smiles>CC(C)(C)OC(=O)NCCN1C(=O)c2cccnc2Sc2cc(C(F)(F)F)ccc21</smiles>

$\mathrm{CDCl}_{3}$

$\begin{array}{llllllllllll}10 & 200 & 190 & 180 & 170 & 160 & 150 & 140 & 130 & 120 & 110 & 100 \\ \mathrm{f} 1(\mathrm{MA})\end{array}$ 


\section{${ }^{1} \mathrm{H}\left(400 \mathrm{MHz}\right.$; in $\left.\mathrm{CDCl}_{3}\right)$ and ${ }^{13} \mathrm{C}\left\{{ }^{1} \mathrm{H}\right\}\left(101 \mathrm{MHz}\right.$; in $\left.\mathrm{CDCl}_{3}\right)$ NMR spectra of compound $9 \mathrm{~m}$}

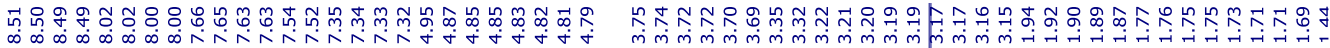
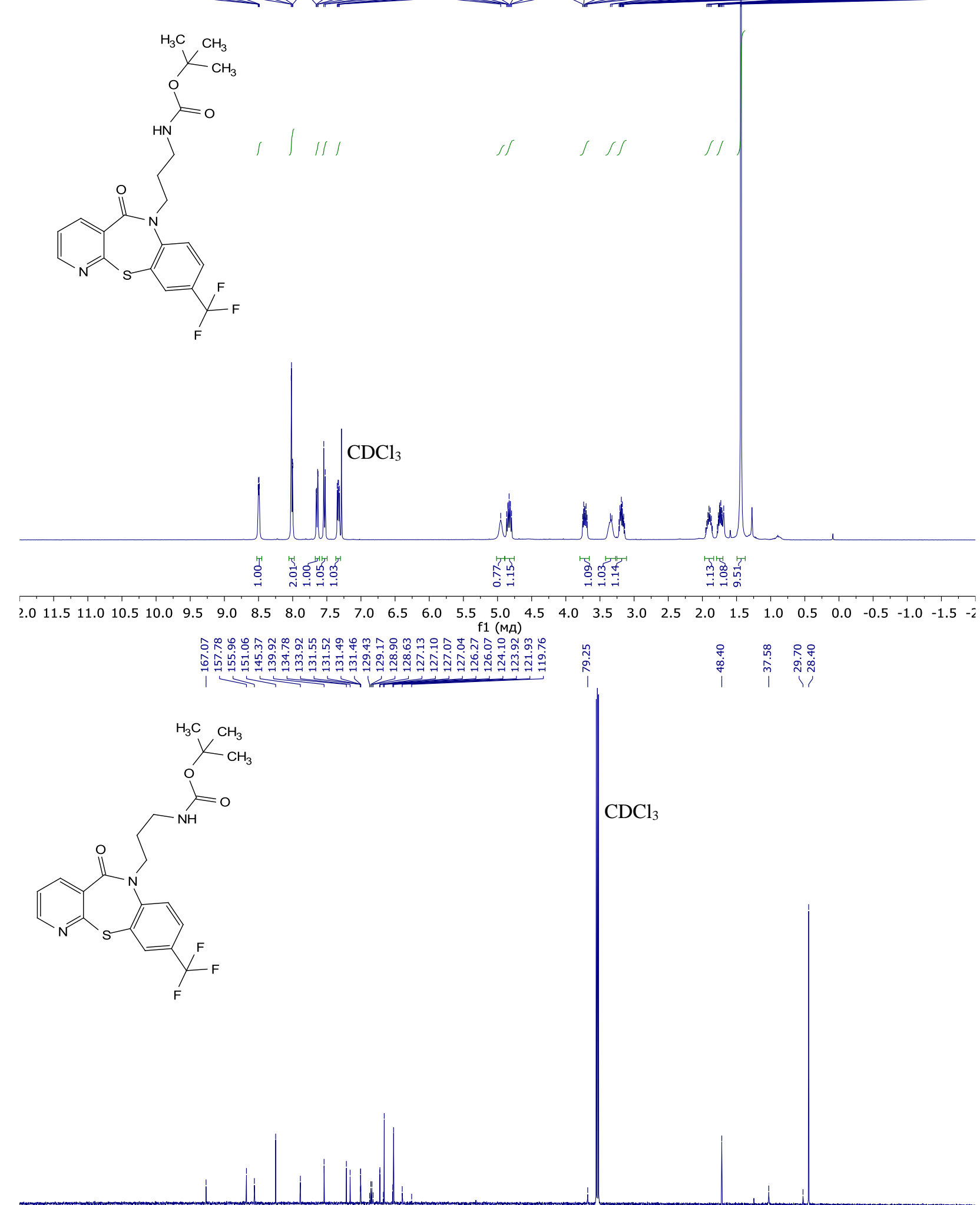

$\begin{array}{llllllllllllllllllllllll}10 & 200 & 190 & 180 & 170 & 160 & 150 & 140 & 130 & 120 & 110 & 100 & 90 & 80 & 70 & 60 & 50 & 40 & 30 & 20 & 10 & 0 & \cdots\end{array}$ 
${ }^{1} \mathrm{H}\left(400 \mathrm{MHz}\right.$; in $\left.\mathrm{CDCl}_{3}\right)$ and ${ }^{13} \mathrm{C}\left\{{ }^{1} \mathrm{H}\right\}\left(101 \mathrm{MHz}\right.$; in $\left.\mathrm{CDCl}_{3}\right)$ NMR spectra of compound 9n
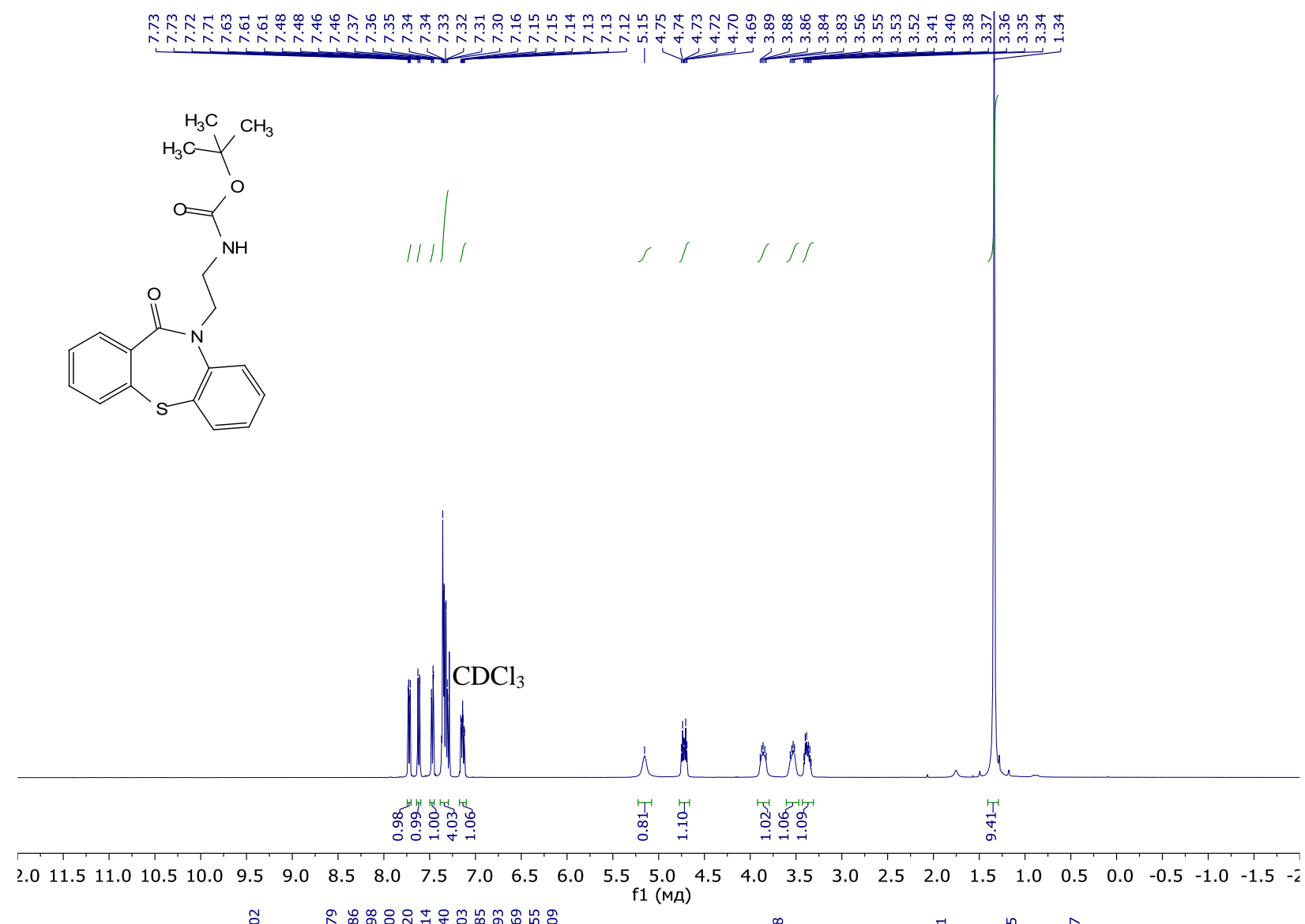

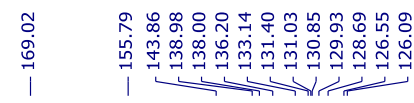<smiles>CC(C)(C)OC(=O)NCCN1C(=O)c2ccccc2Sc2ccccc21</smiles>

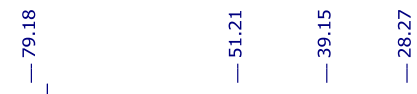

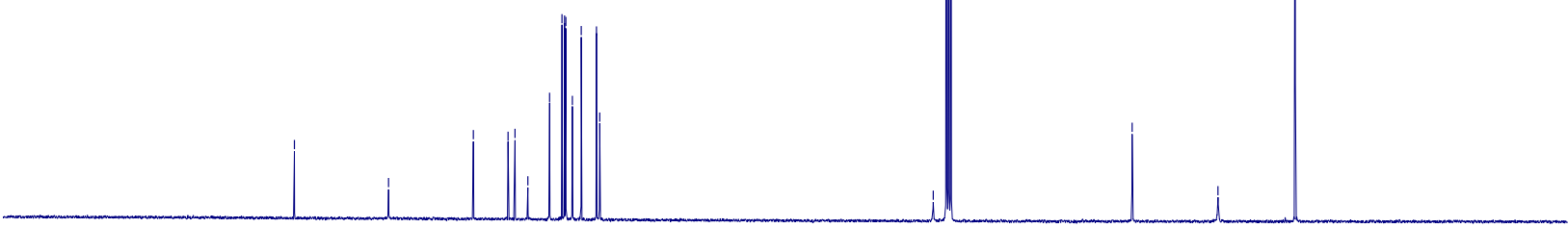

10

$\begin{array}{lllllllllll}200 & 190 & 180 & 170 & 160 & 150 & 140 & 130 & 120 & 110 \begin{array}{c}100 \\ \mathrm{f} 1(\mathrm{MA})\end{array}\end{array}$ $\mathrm{CDCl}_{3}$ 
${ }^{1} \mathrm{H}\left(400 \mathrm{MHz}\right.$; in $\left.\mathrm{CDCl}_{3}\right)$ and ${ }^{13} \mathrm{C}\left\{{ }^{1} \mathrm{H}\right\}\left(101 \mathrm{MHz}\right.$; in $\left.\mathrm{CDCl}_{3}\right) \mathrm{NMR}$ spectra of compound 11a

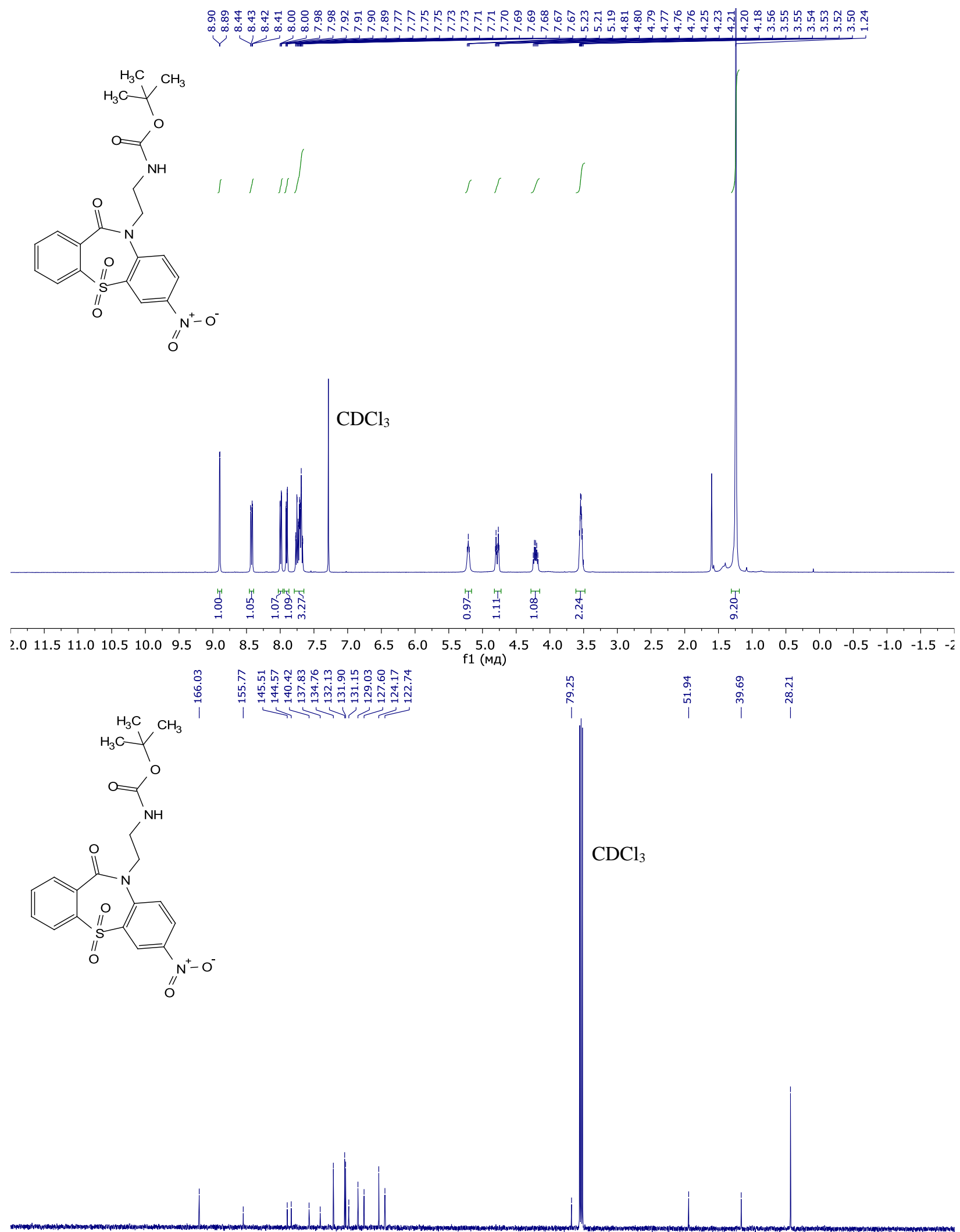

\begin{tabular}{lllllllllllllllllllllllll}
\hline 10 & 200 & 190 & 180 & 170 & 160 & 150 & 140 & 130 & 120 & 110 & 100 & 90 & 80 & 70 & 60 & 50 & 40 & 30 & 20 & 10 & 0 & -
\end{tabular} 
${ }^{1} \mathrm{H}\left(400 \mathrm{MHz}\right.$; in $\left.\mathrm{CDCl}_{3}\right)$ and ${ }^{13} \mathrm{C}\left\{{ }^{1} \mathrm{H}\right\}\left(101 \mathrm{MHz}\right.$; in $\left.\mathrm{CDCl}_{3}\right)$ NMR spectra of compound 11b
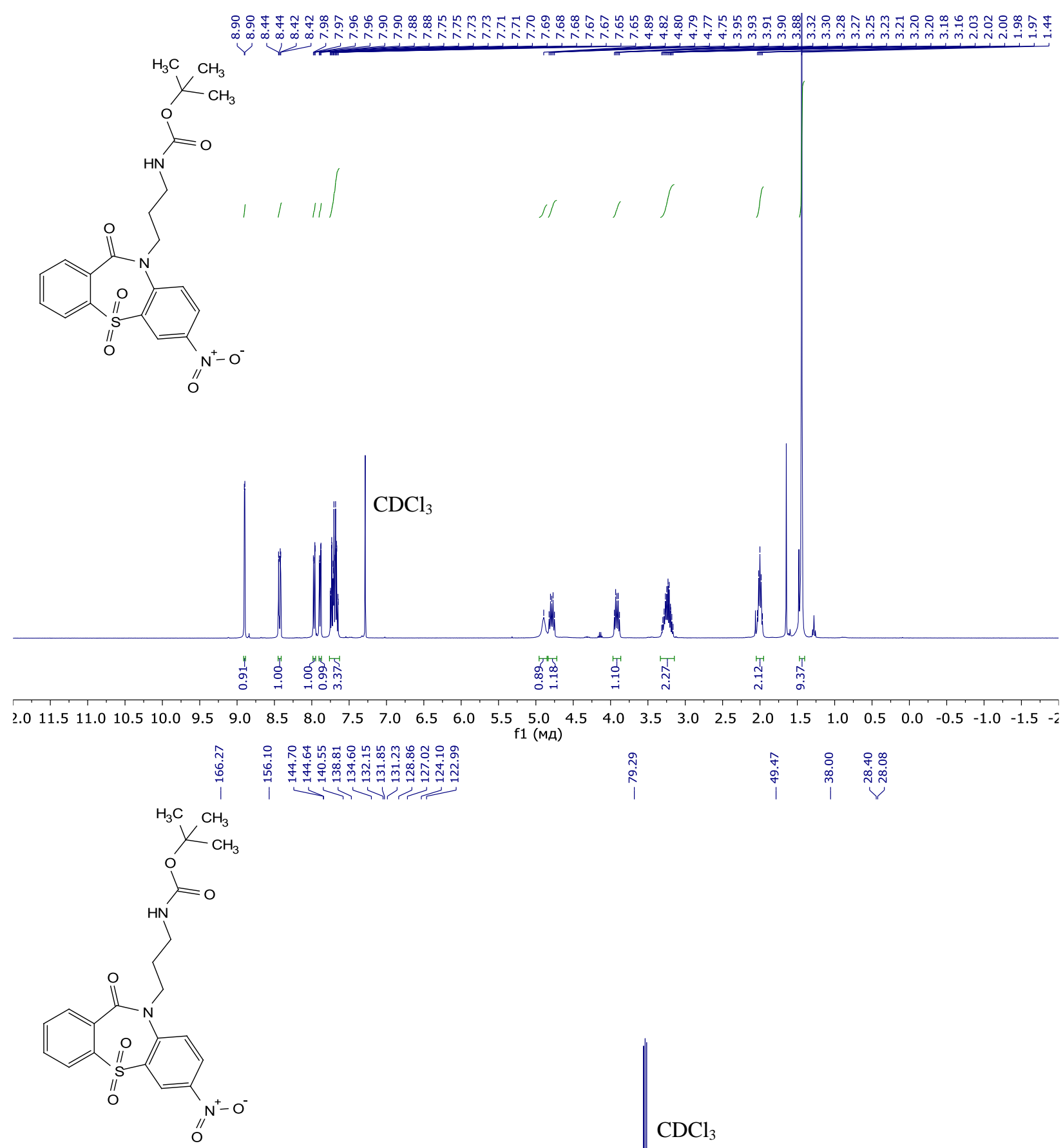

$\mathrm{CDCl}_{3}$

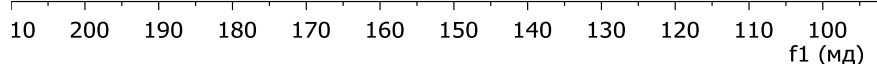


${ }^{1} \mathrm{H}\left(400 \mathrm{MHz}\right.$; in $\left.\mathrm{CDCl}_{3}\right)$ and ${ }^{13} \mathrm{C}\left\{{ }^{1} \mathrm{H}\right\}\left(101 \mathrm{MHz}\right.$; in $\left.\mathrm{CDCl}_{3}\right)$ NMR spectra of compound 11c
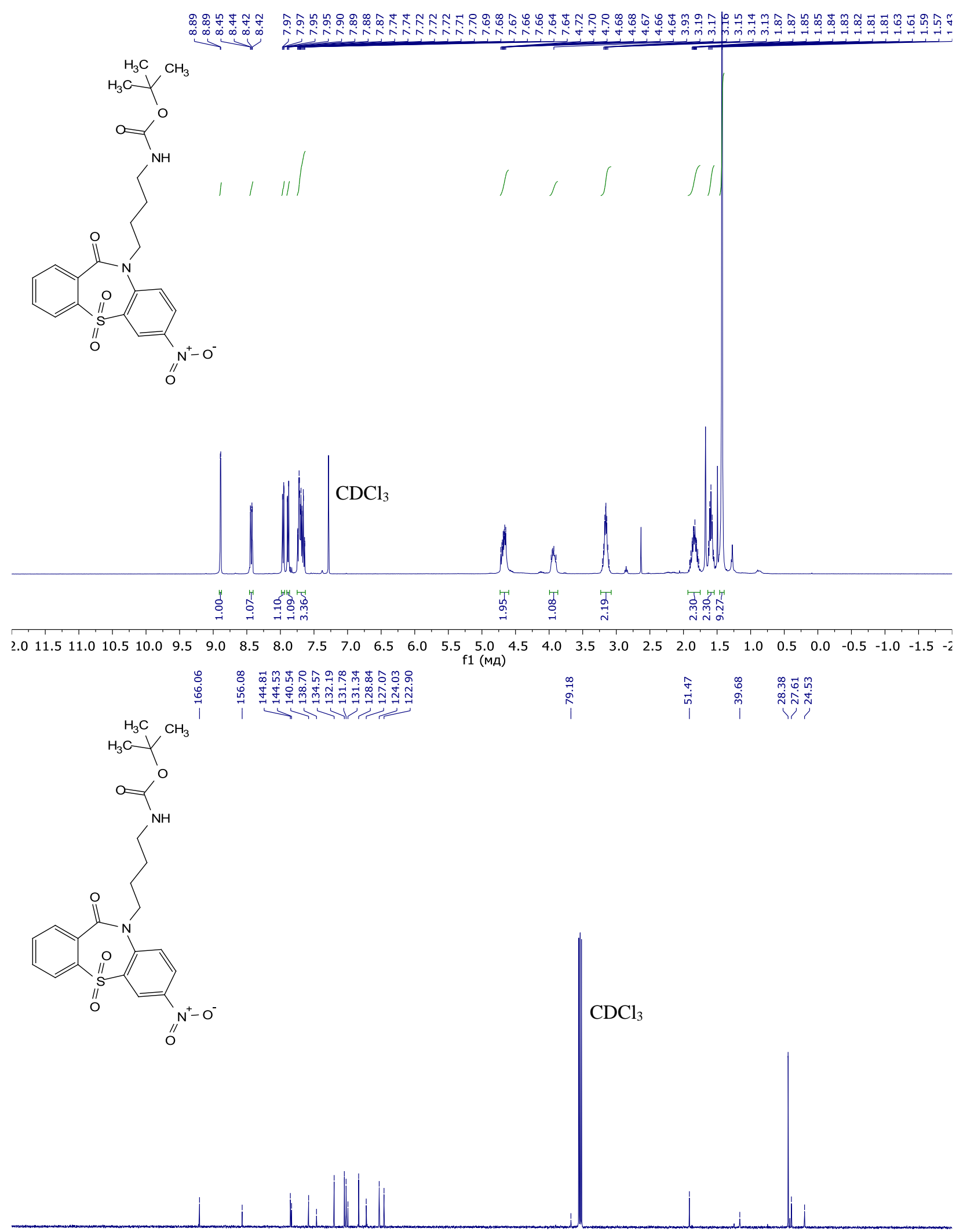

\begin{tabular}{lllllllllllllllllllllll}
\hline 10 & 200 & 190 & 180 & 170 & 160 & 150 & 140 & 130 & 120 & 110 & $\begin{array}{c}100 \\
\mathrm{f} 1(\mathrm{MA})\end{array}$ & 90 & 80 & 70 & 60 & 50 & 40 & 30 & 20 & 10 & 0 & -
\end{tabular}

( 
${ }^{1} \mathrm{H}\left(400 \mathrm{MHz}\right.$; in $\left.\mathrm{CDCl}_{3}\right)$ and ${ }^{13} \mathrm{C}\left\{{ }^{1} \mathrm{H}\right\}\left(101 \mathrm{MHz}\right.$; in $\left.\mathrm{CDCl}_{3}\right)$ NMR spectra of compound 11d

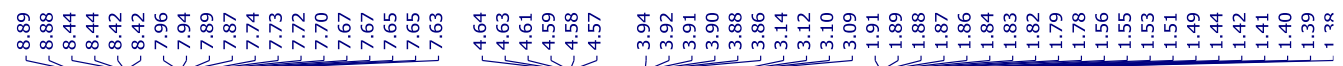
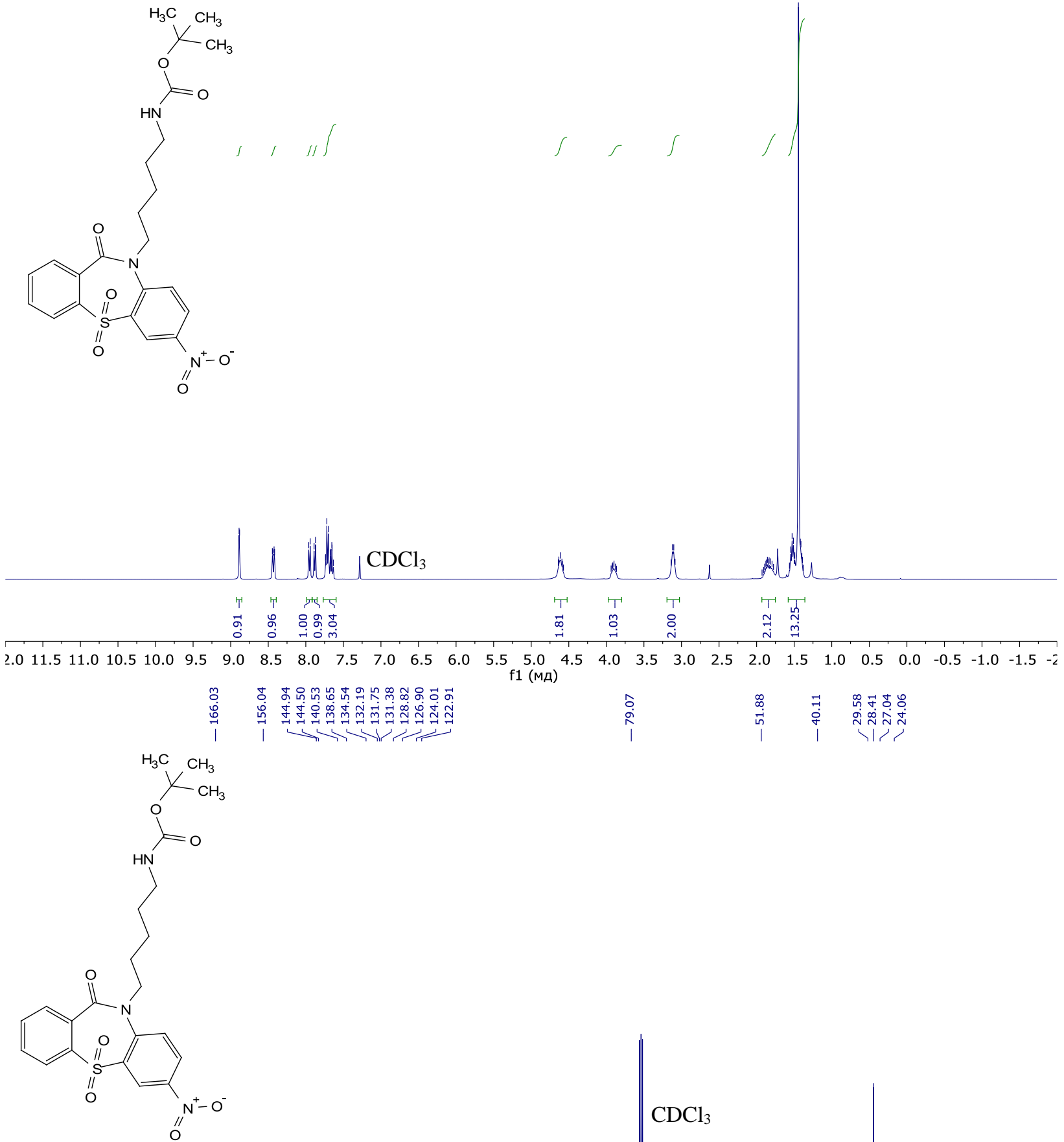

o

$\mathrm{CDCl}_{3}$
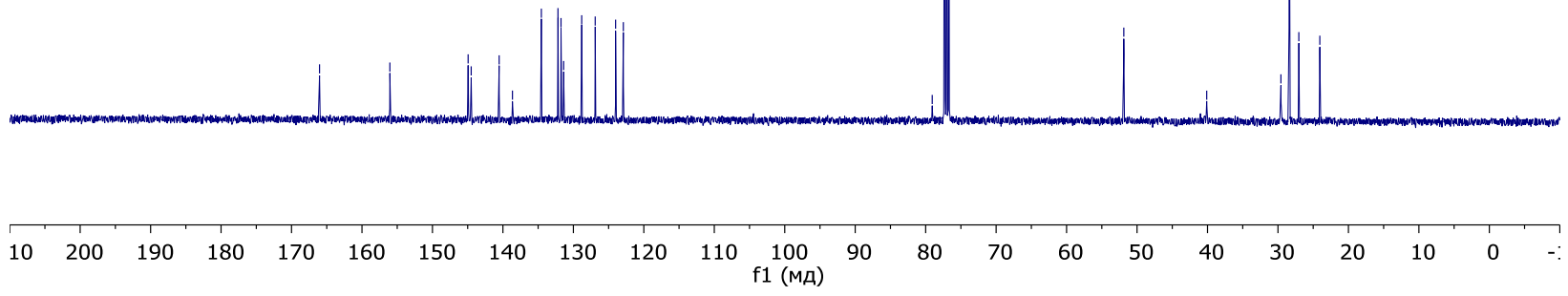

S45 
${ }^{1} \mathrm{H}\left(400 \mathrm{MHz}\right.$; in $\left.\mathrm{CDCl}_{3}\right)$ and ${ }^{13} \mathrm{C}\left\{{ }^{1} \mathrm{H}\right\}\left(101 \mathrm{MHz}\right.$; in $\left.\mathrm{CDCl}_{3}\right)$ NMR spectra of compound $11 \mathrm{e}$

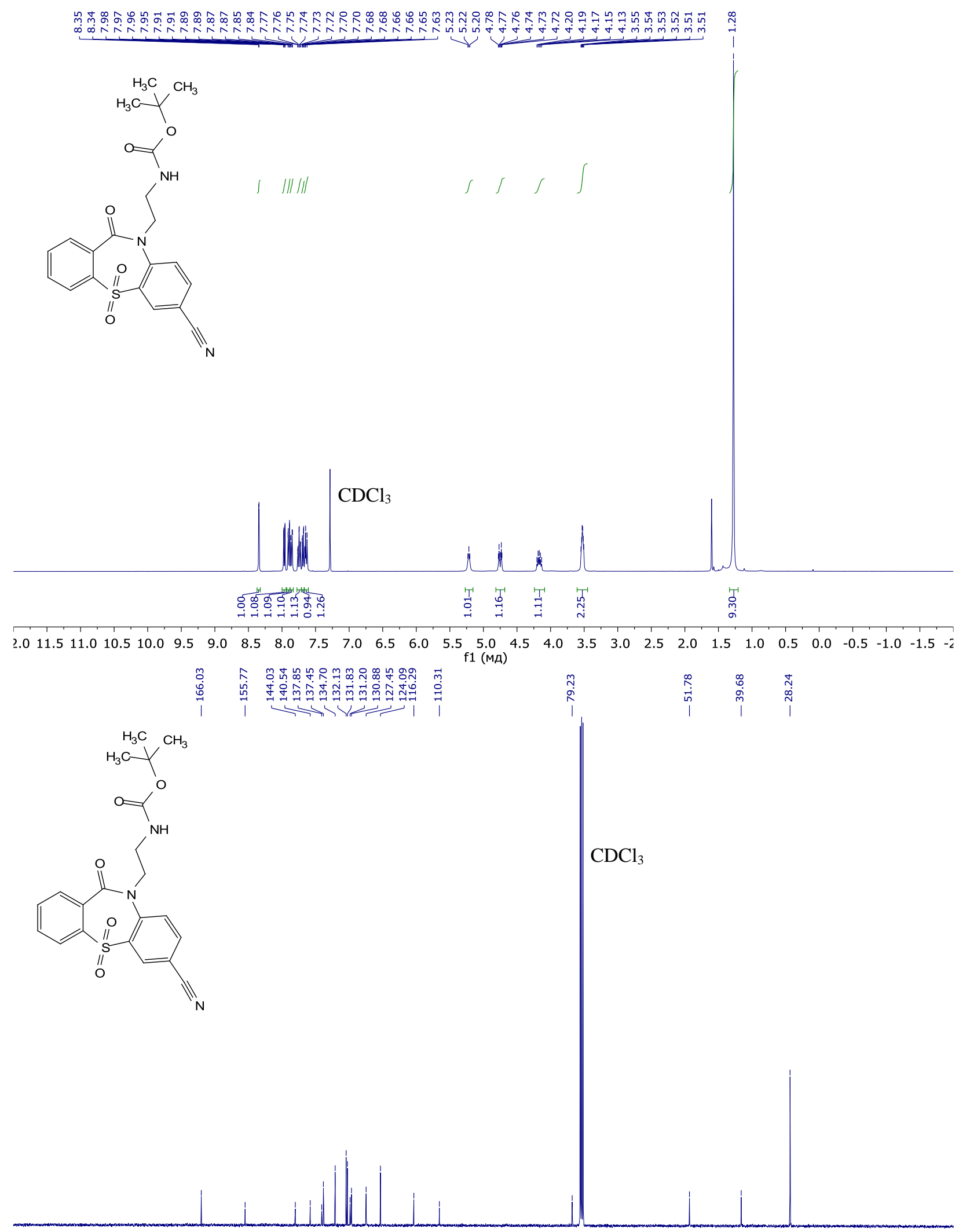

\begin{tabular}{lllllllllllllllllllllll}
\hline 10 & 200 & 190 & 180 & 170 & 160 & 150 & 140 & 130 & 120 & 110 & $\begin{array}{c}100 \\
\mathrm{f} 1(\mathrm{MA})\end{array}$ & 90 & 80 & 70 & 60 & 50 & 40 & 30 & 20 & 10 & 0 & -
\end{tabular} (Mम) 
${ }^{1} \mathrm{H}\left(400 \mathrm{MHz}\right.$; in $\left.\mathrm{CDCl}_{3}\right)$ and ${ }^{13} \mathrm{C}\left\{{ }^{1} \mathrm{H}\right\}\left(101 \mathrm{MHz}\right.$; in $\left.\mathrm{CDCl}_{3}\right)$ NMR spectra of compound $11 \mathrm{f}$

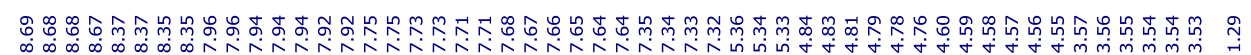
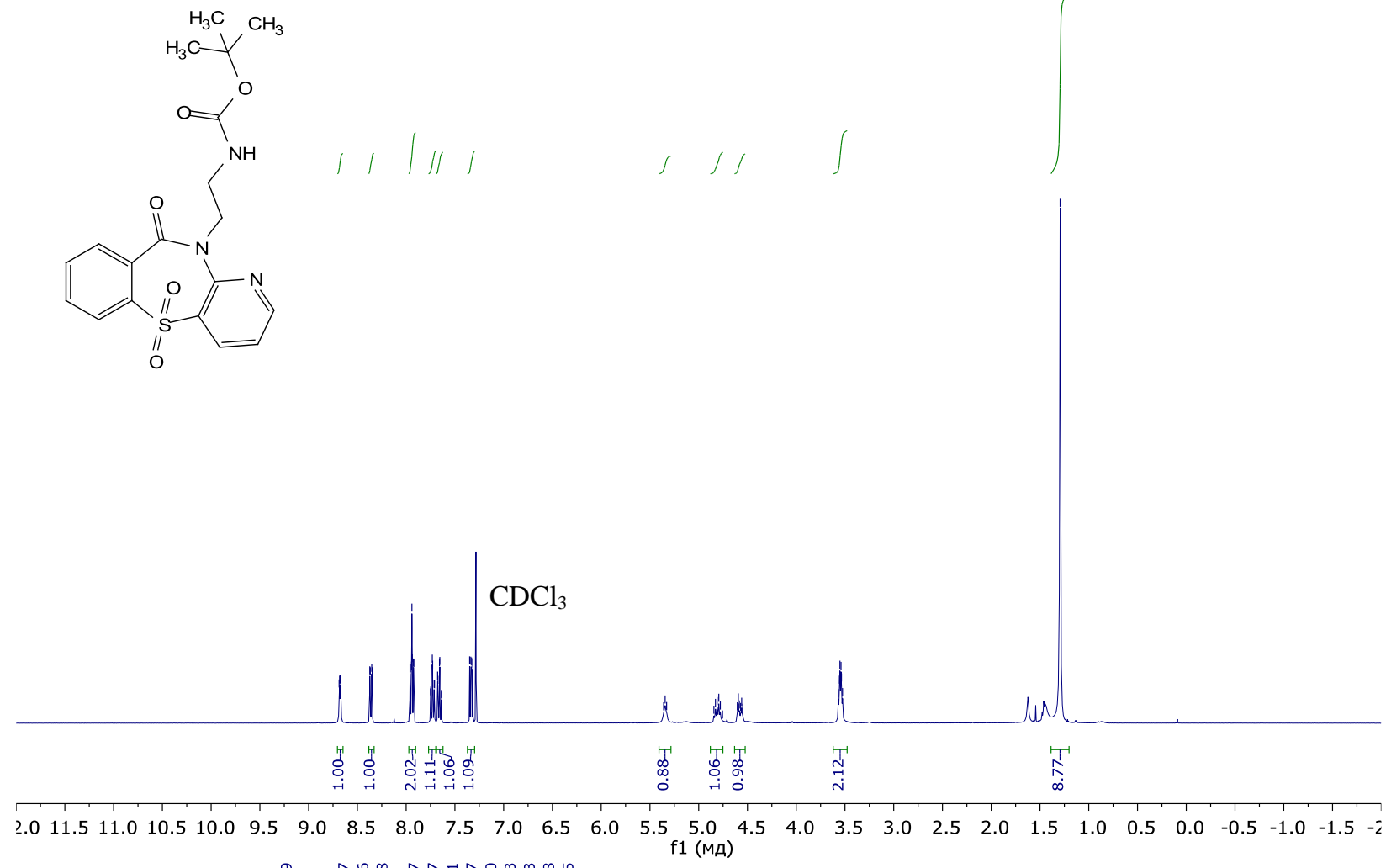

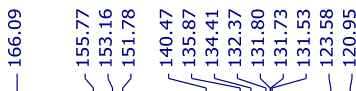
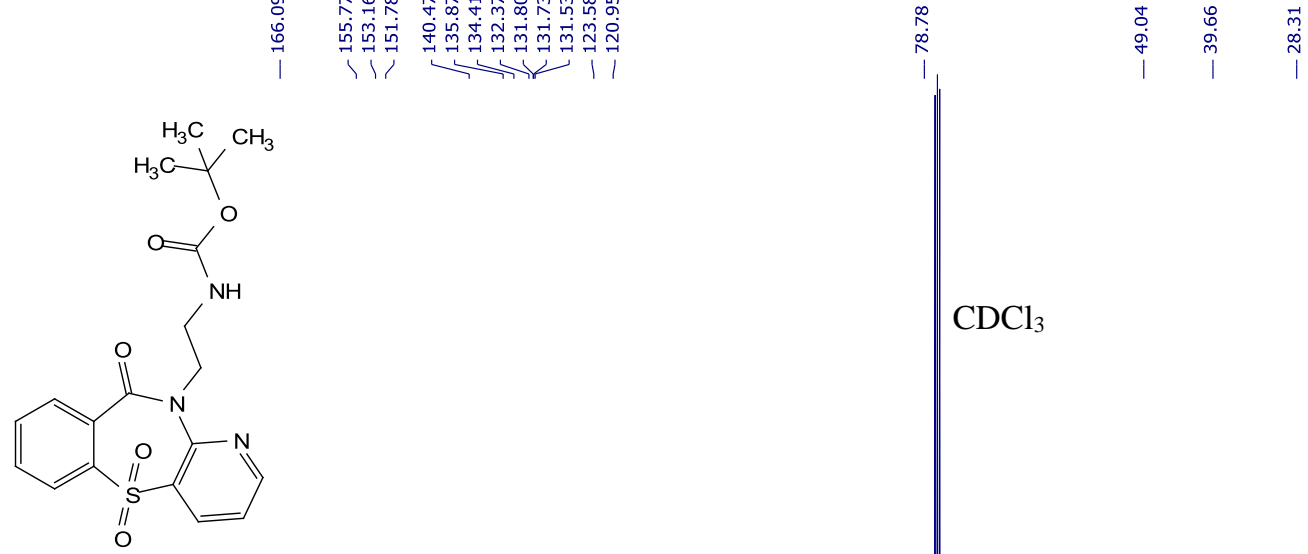

$\mathrm{CDCl}_{3}$

$\begin{array}{llllllllllll}10 & 200 & 190 & 180 & 170 & 160 & 150 & 140 & 130 & 120 & 110 & 100 \\ \mathrm{f} 1(\mathrm{Mg})\end{array}$ 
${ }^{1} \mathrm{H}\left(400 \mathrm{MHz}\right.$; in $\left.\mathrm{CDCl}_{3}\right)$ and ${ }^{13} \mathrm{C}\left\{{ }^{1} \mathrm{H}\right\}\left(101 \mathrm{MHz}\right.$; in $\left.\mathrm{CDCl}_{3}\right)$ NMR spectra of compound $11 \mathrm{~g}$

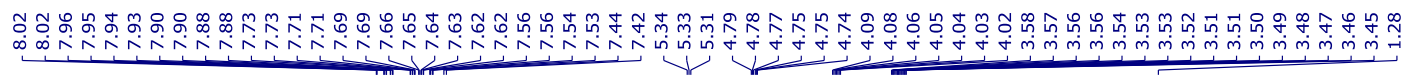

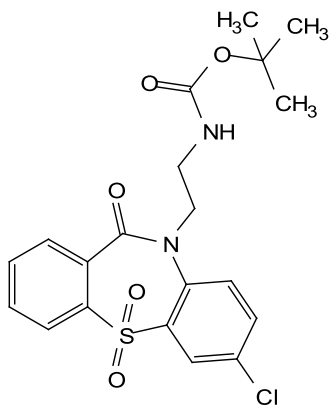

|II III
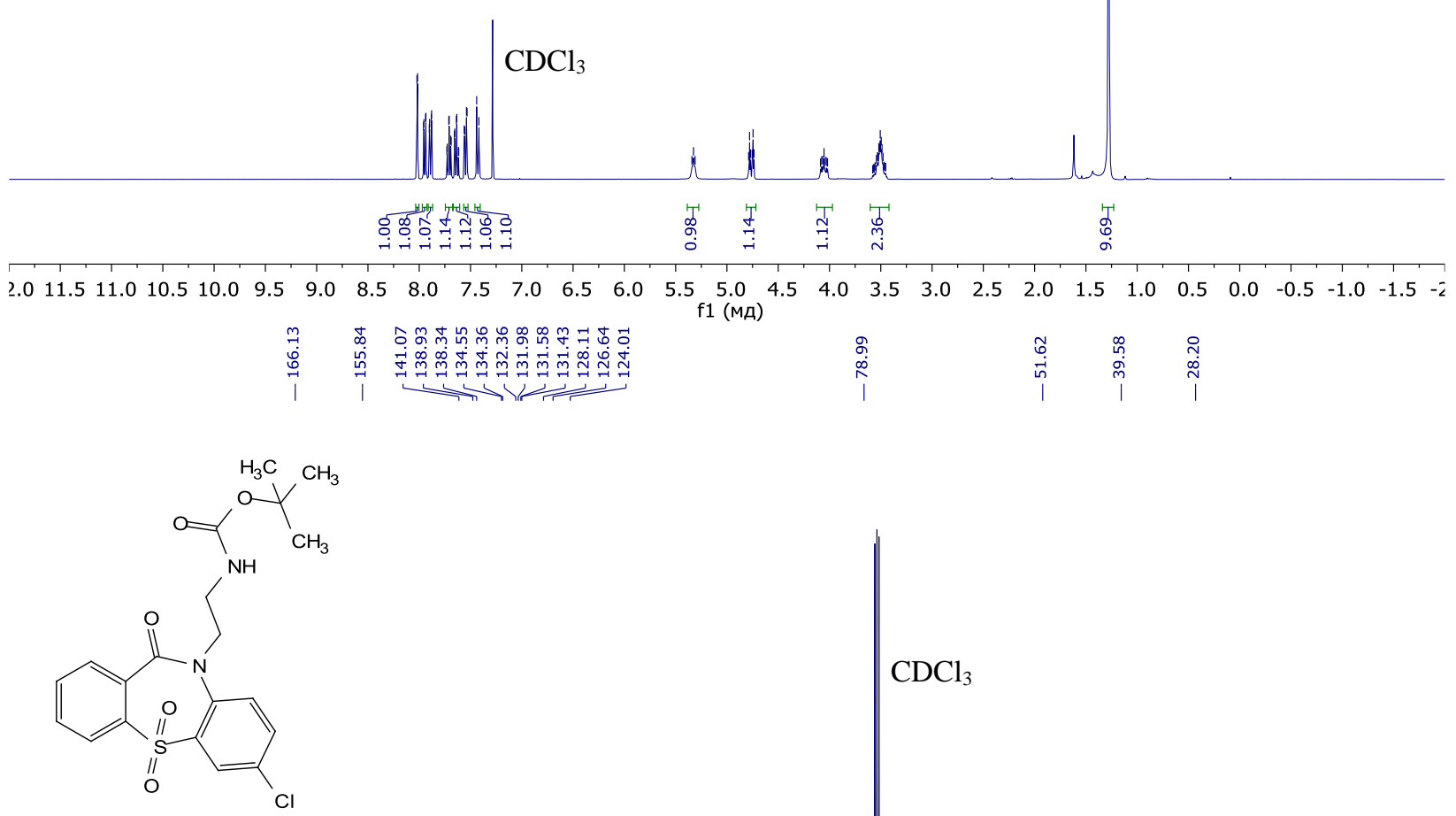

$\mathrm{CDCl}_{3}$
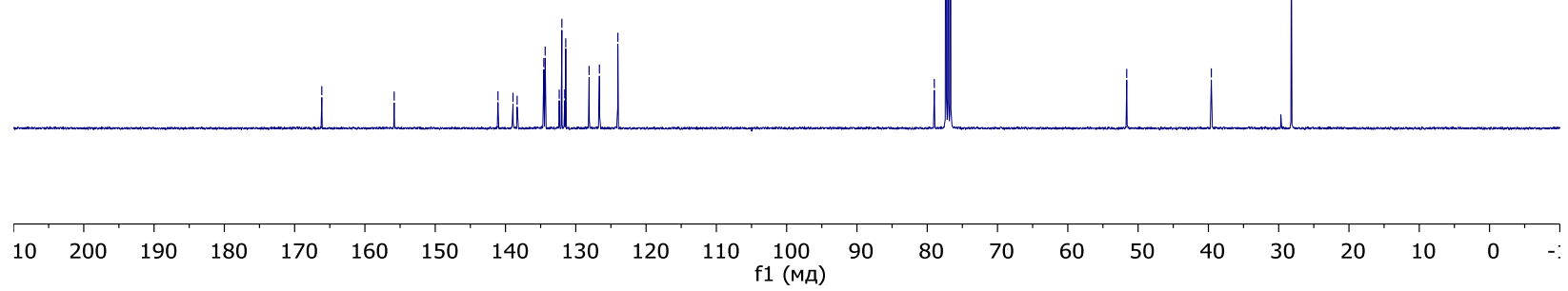

S48 
${ }^{1} \mathrm{H}\left(400 \mathrm{MHz}\right.$; in $\left.\mathrm{CDCl}_{3}\right)$ and ${ }^{13} \mathrm{C}\left\{{ }^{1} \mathrm{H}\right\}\left(101 \mathrm{MHz}\right.$; in $\left.\mathrm{CDCl}_{3}\right) \mathrm{NMR}$ spectra of compound $11 \mathrm{~h}$

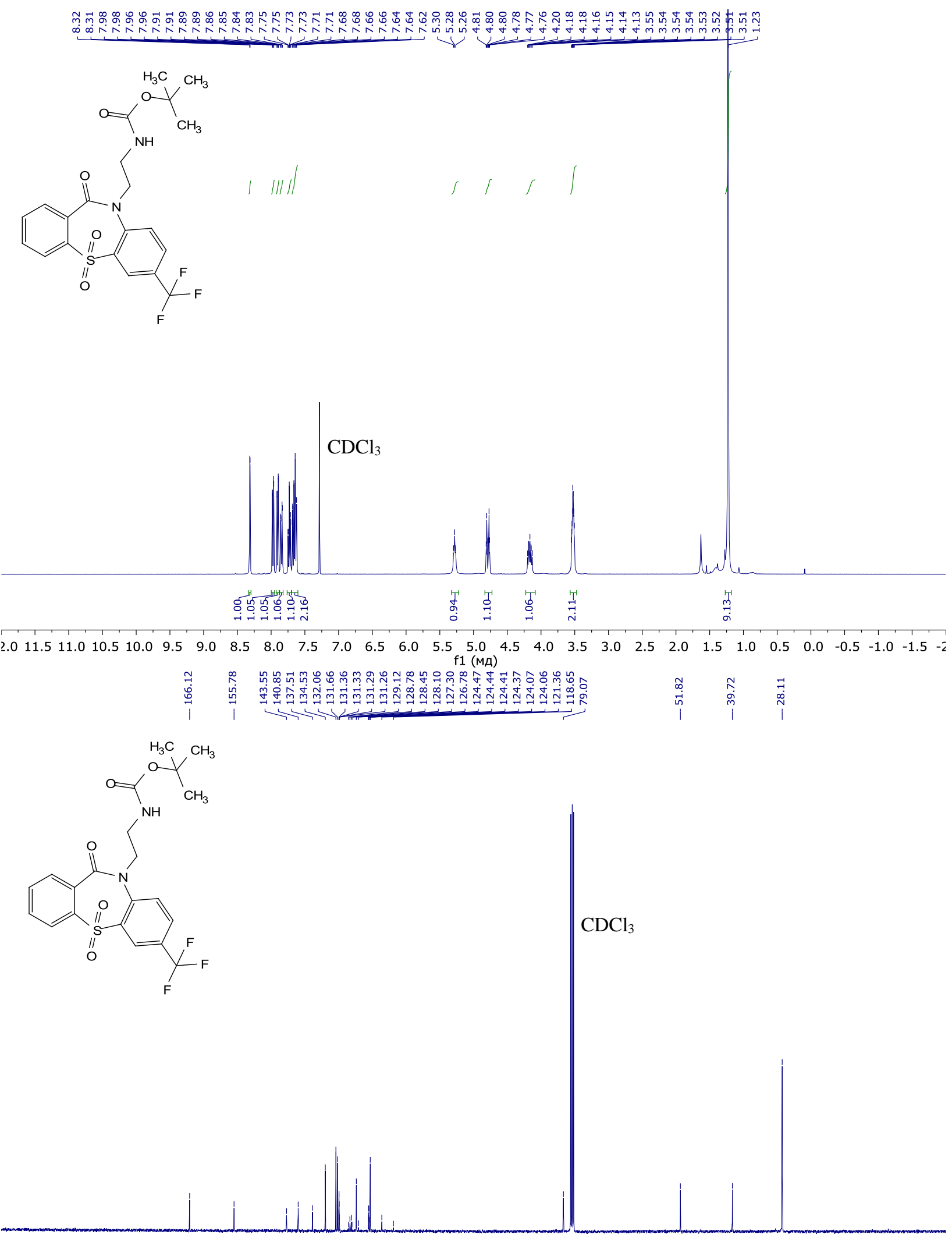

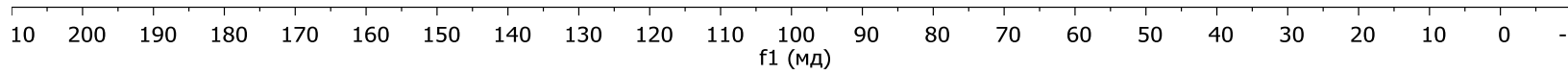


${ }^{1} \mathrm{H}\left(400 \mathrm{MHz}\right.$; in $\left.\mathrm{CDCl}_{3}\right)$ and ${ }^{13} \mathrm{C}\left\{{ }^{1} \mathrm{H}\right\}\left(101 \mathrm{MHz}\right.$; in $\left.\mathrm{CDCl}_{3}\right)$ NMR spectra of compound $11 \mathrm{i}$
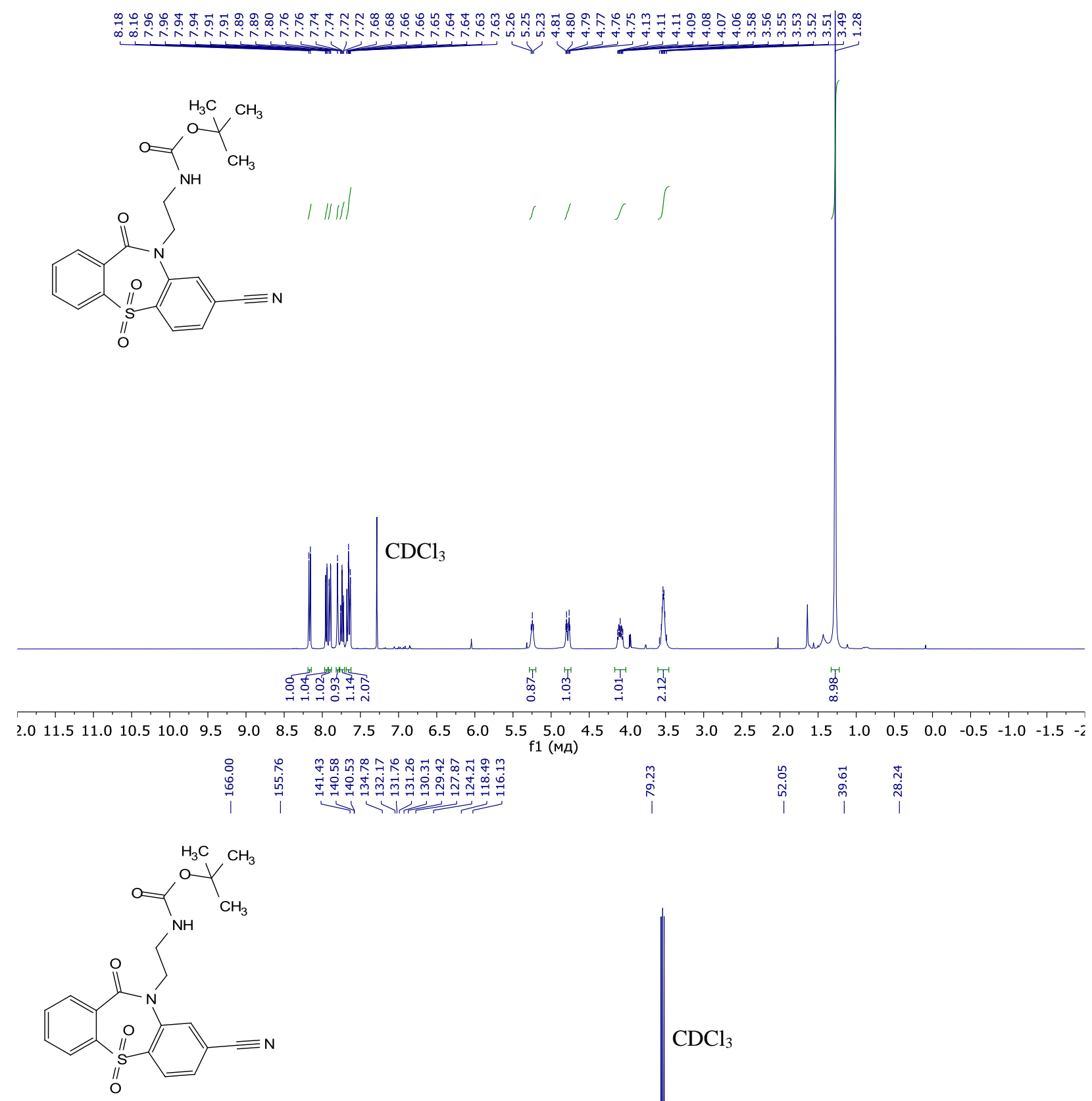

$\mathrm{CDCl}_{3}$

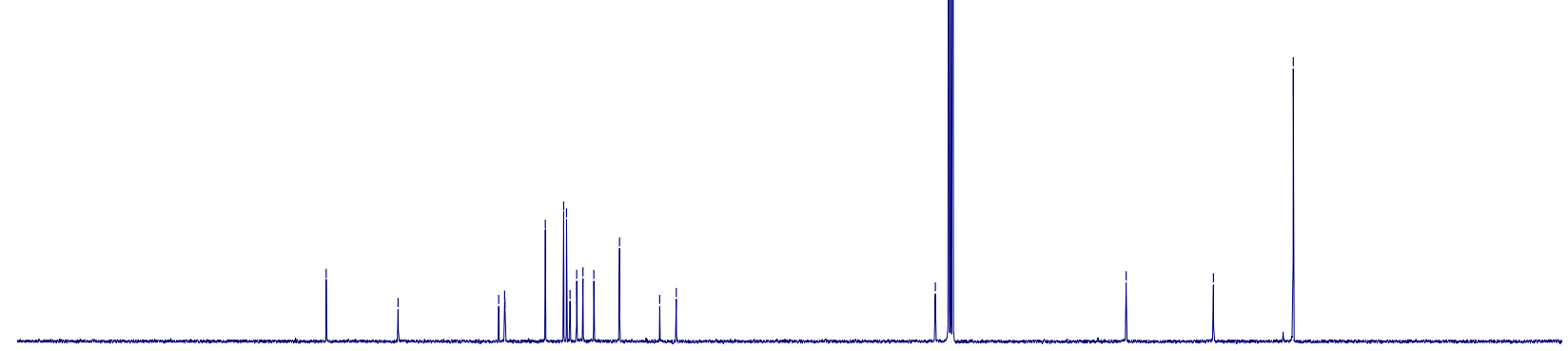

$\begin{array}{llllllllllllllllllllllllll}10 & 200 & 190 & 180 & 170 & 160 & 150 & 140 & 130 & 120 & 110 & 100 & 90 & 80 & 70 & 60 & 50 & 40 & 30 & 20 & 10 & 0 & -\end{array}$ 
${ }^{1} \mathrm{H}\left(400 \mathrm{MHz}\right.$; in $\left.\mathrm{CDCl}_{3}\right)$ and ${ }^{13} \mathrm{C}\left\{{ }^{1} \mathrm{H}\right\}\left(101 \mathrm{MHz}\right.$; in $\left.\mathrm{CDCl}_{3}\right)$ NMR spectra of compound $11 \mathrm{j}$

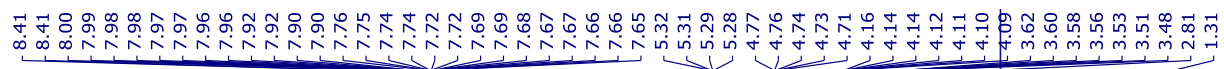
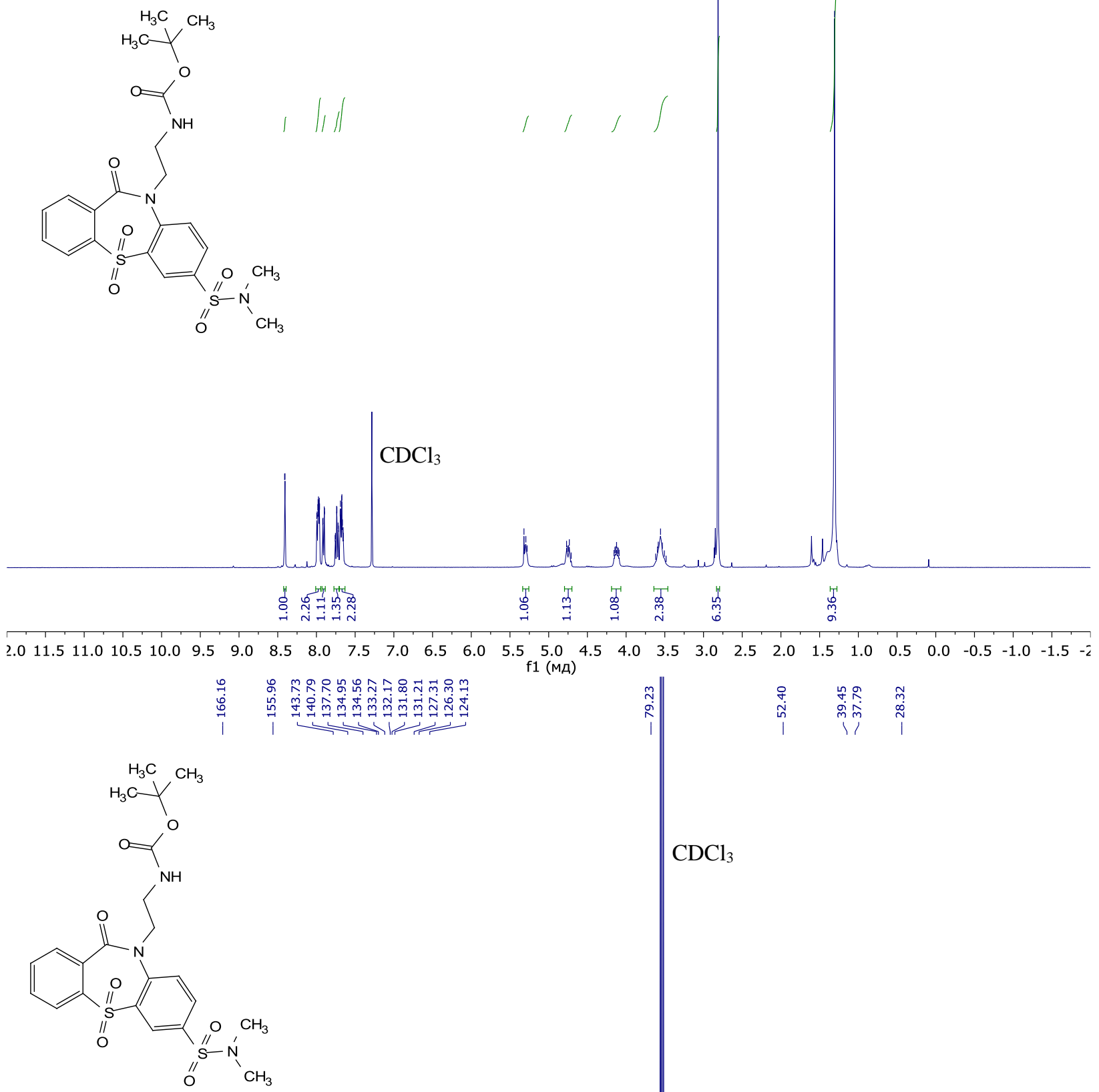

$\mathrm{CDCl}_{3}$

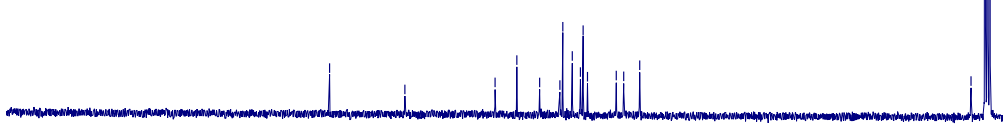

in

. 
${ }^{1} \mathrm{H}\left(400 \mathrm{MHz}\right.$; in $\left.\mathrm{CDCl}_{3}\right)$ and ${ }^{13} \mathrm{C}\left\{{ }^{1} \mathrm{H}\right\}\left(101 \mathrm{MHz}\right.$; in $\left.\mathrm{CDCl}_{3}\right)$ NMR spectra of compound $11 \mathrm{k}$
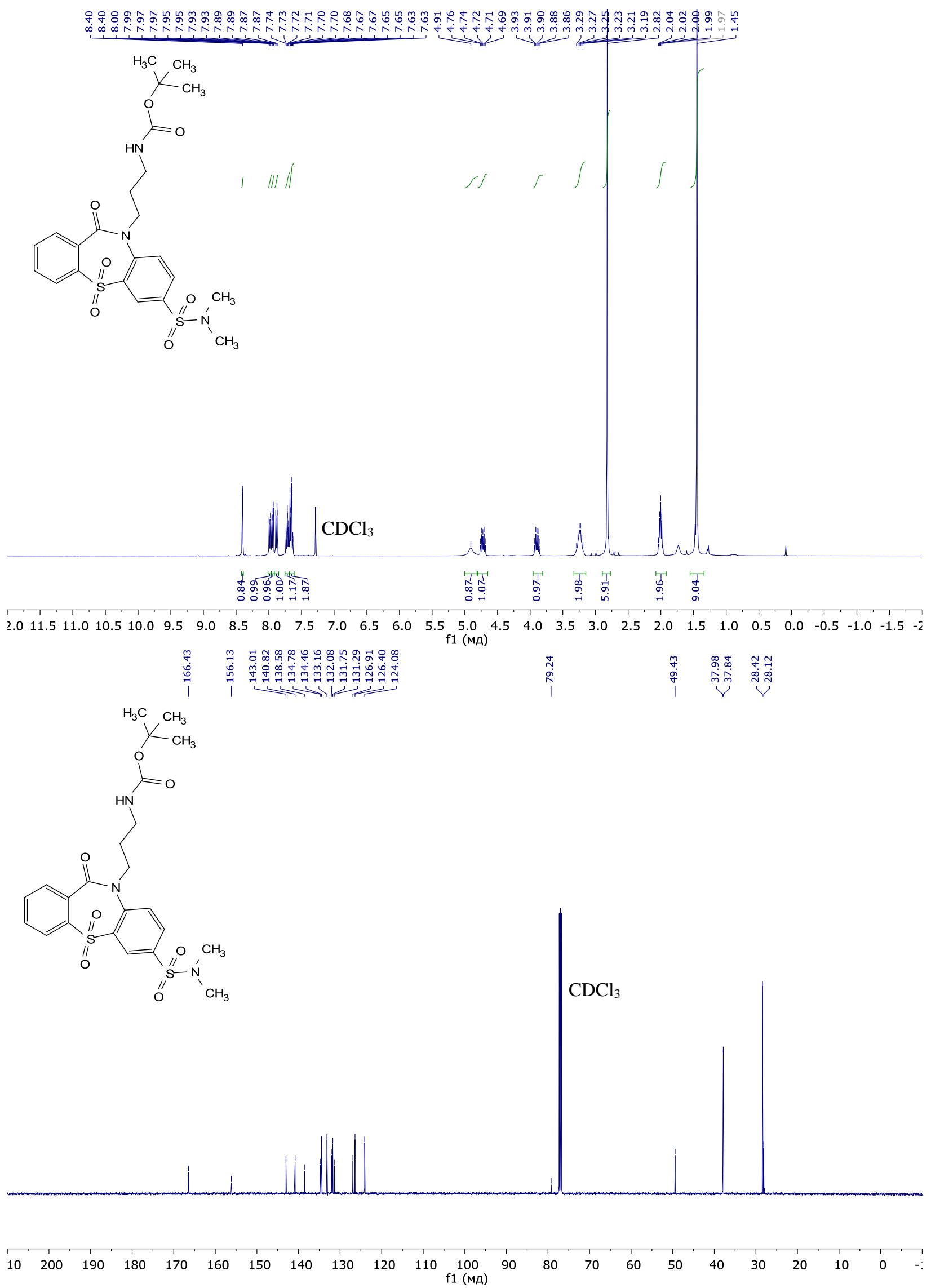

S52 
${ }^{1} \mathrm{H}\left(400 \mathrm{MHz}\right.$; in $\left.\mathrm{CDCl}_{3}\right)$ and ${ }^{13} \mathrm{C}\left\{{ }^{1} \mathrm{H}\right\}\left(101 \mathrm{MHz}\right.$; in $\left.\mathrm{CDCl}_{3}\right)$ NMR spectra of compound 111
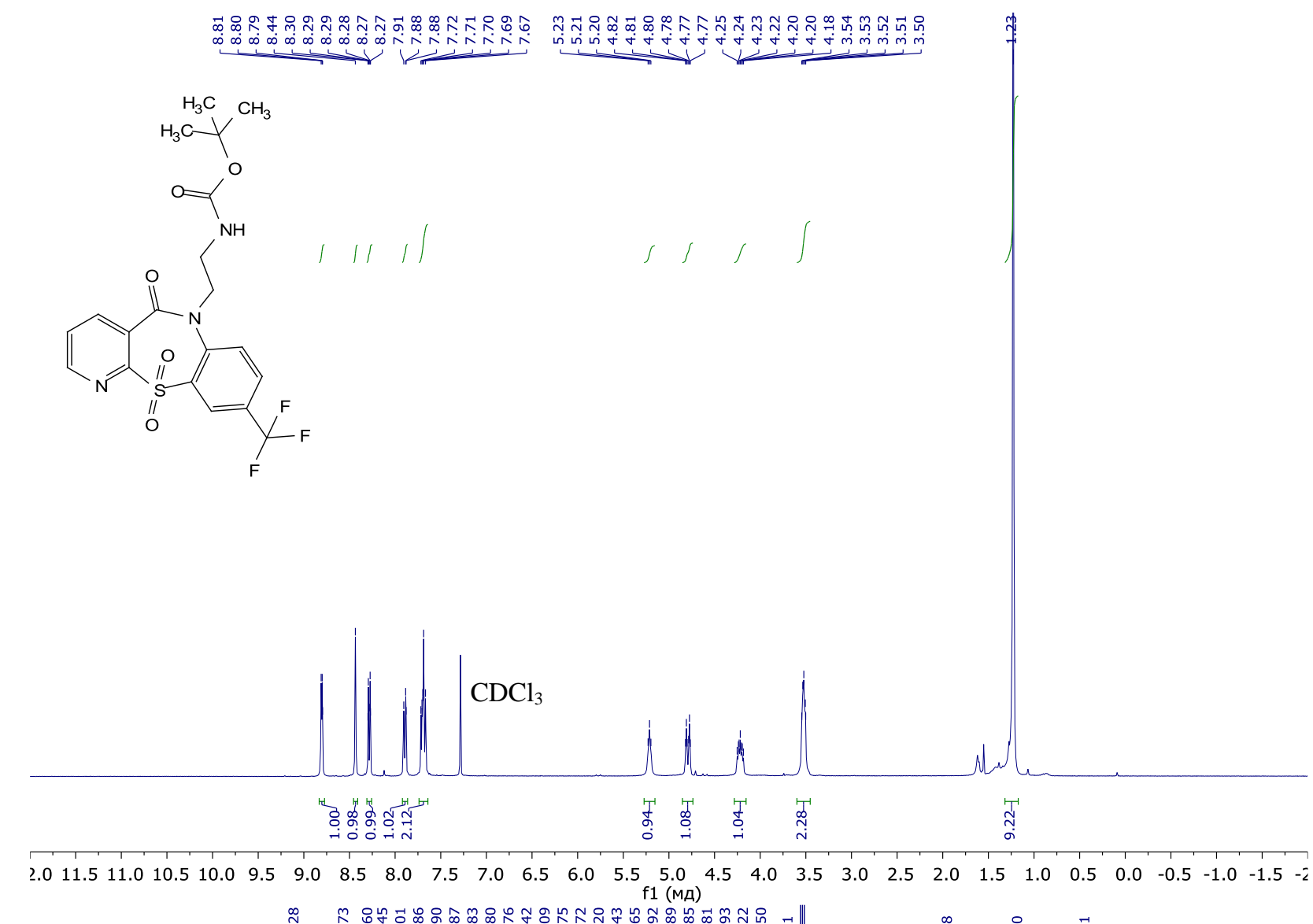

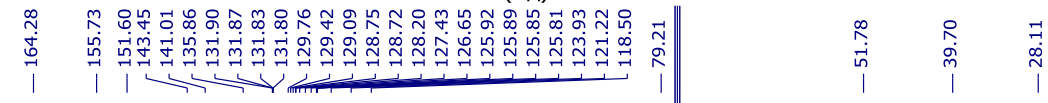

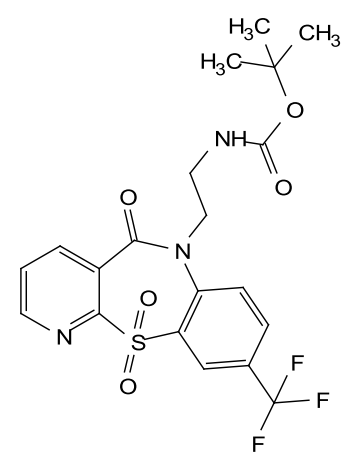

$\mathrm{CDCl}_{3}$

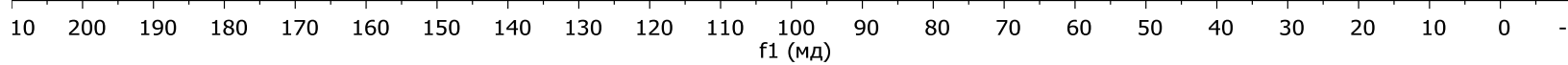


${ }^{1} \mathrm{H}\left(400 \mathrm{MHz}\right.$; in $\left.\mathrm{CDCl}_{3}\right)$ and ${ }^{13} \mathrm{C}\left\{{ }^{1} \mathrm{H}\right\}\left(101 \mathrm{MHz}\right.$; in $\left.\mathrm{CDCl}_{3}\right) \mathrm{NMR}$ spectra of compound $11 \mathrm{~m}$

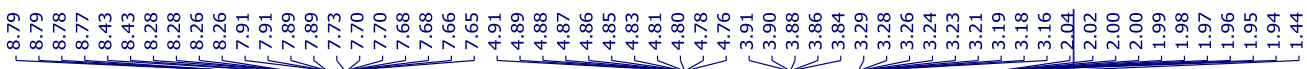
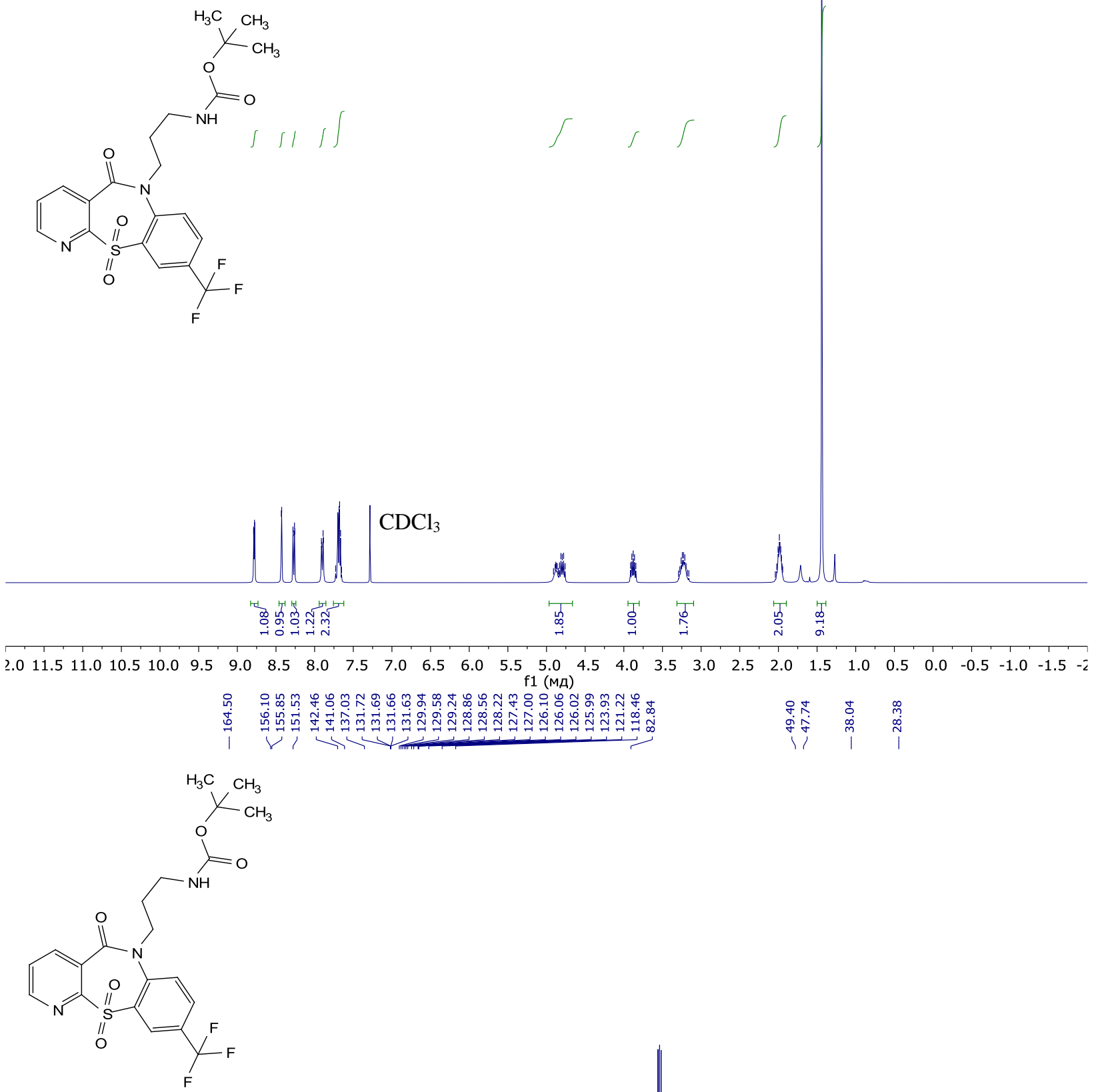

$\mathrm{CDCl}_{3}$

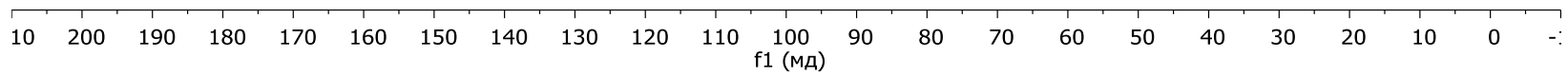

S54 
${ }^{1} \mathrm{H}\left(400 \mathrm{MHz}\right.$; in $\left.\mathrm{CDCl}_{3}\right)$ and ${ }^{13} \mathrm{C}\left\{{ }^{1} \mathrm{H}\right\}\left(101 \mathrm{MHz}\right.$; in $\left.\mathrm{CDCl}_{3}\right)$ NMR spectra of compound 11n

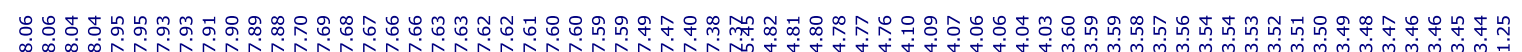<smiles>CC(C)(C)OC(=O)NCCN1C(=O)c2ccccc2S(=O)(=O)c2ccccc21</smiles><smiles>[C]1[C+]=CC=C1</smiles><smiles>CCCC</smiles>

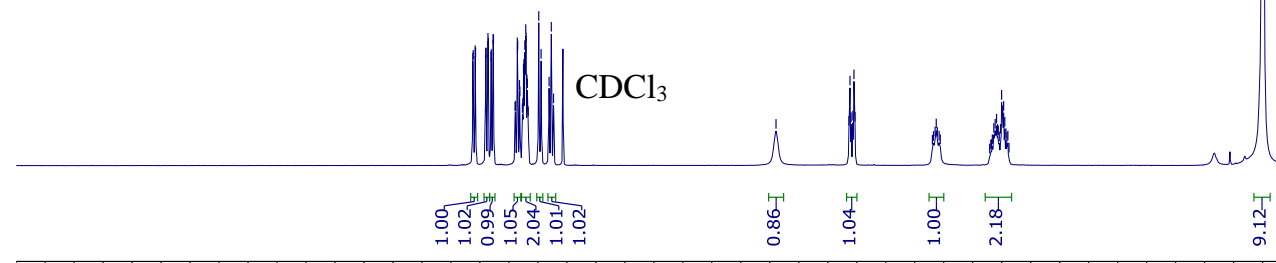

$\begin{array}{llllllllllllllllllllllllllllllllllllllll}2.0 & 11.5 & 11.0 & 10.5 & 10.0 & 9.5 & 9.0 & 8.5 & 8.0 & 7.5 & 7.0 & 6.5 & 6.0 & 5.5 & 5.0 & 4.5 & 4.0 & 3.5 & 3.0 & 2.5 & 2.0 & 1.5 & 1.0 & 0.5 & 0.0 & -0.5 & -1.0 & -1.5 & -2\end{array}$

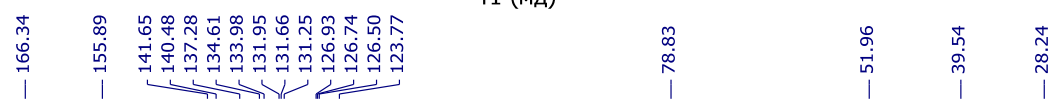<smiles>CC(C)(C)OC(=O)NCCN1C(=O)c2ccccc2S(=O)(=O)c2ccccc21</smiles>
$\mathrm{CDCl}_{3}$

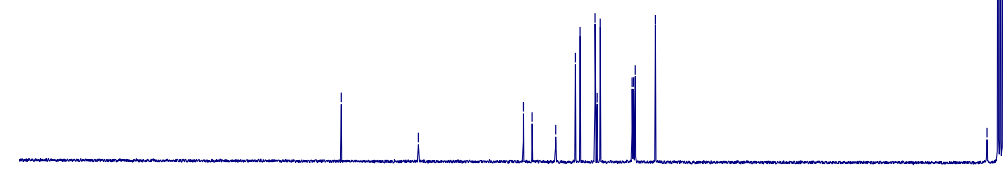

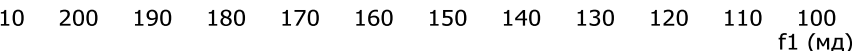


${ }^{1} \mathrm{H}$ (400 MHz; in DMSO- $\left.d_{6}\right)$ and ${ }^{13} \mathrm{C}\left\{{ }^{1} \mathrm{H}\right\}\left(101 \mathrm{MHz}\right.$; in DMSO- $\left.d_{6}\right)$ NMR spectra of compound 12a

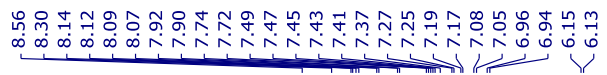
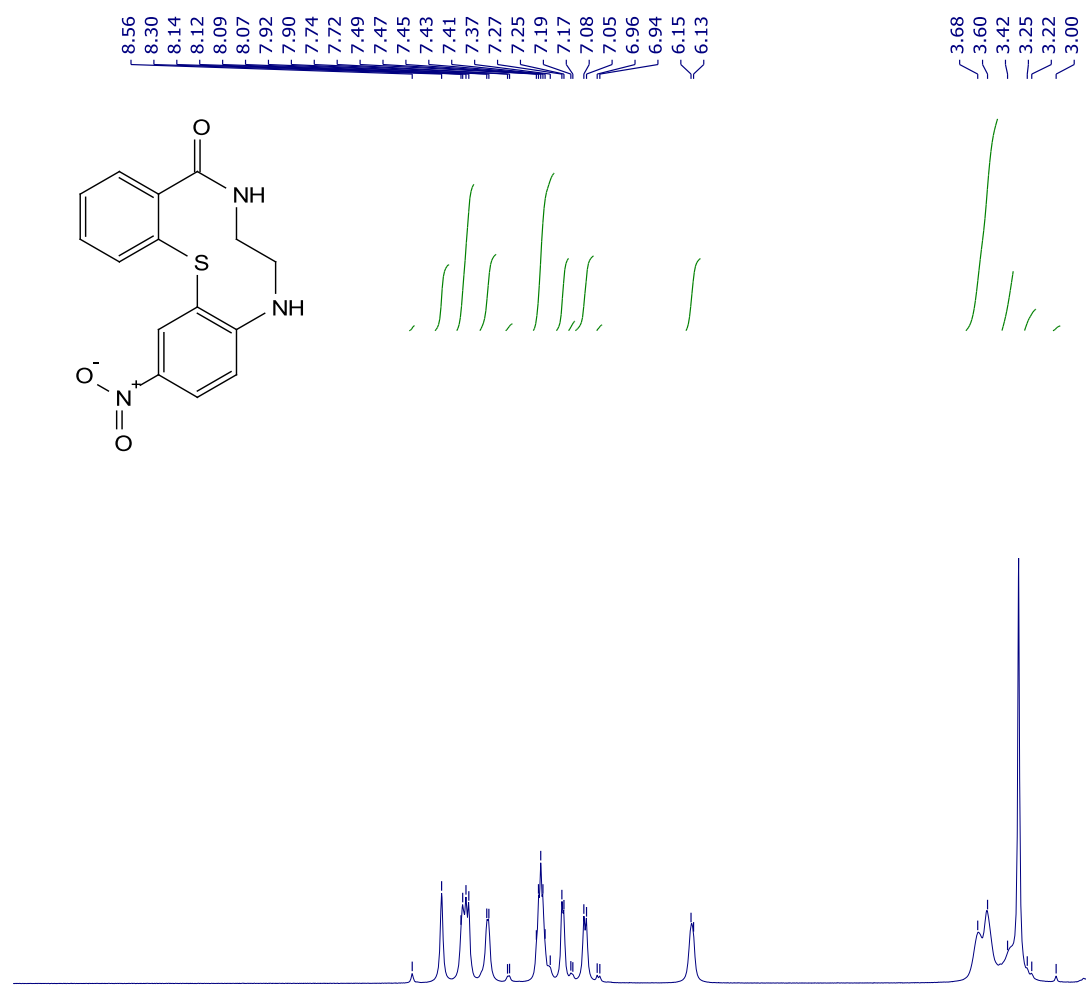

DMSO- $d_{6}$

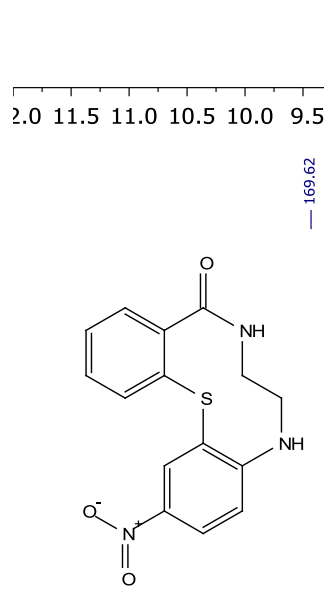

DMSO- $d_{6}$
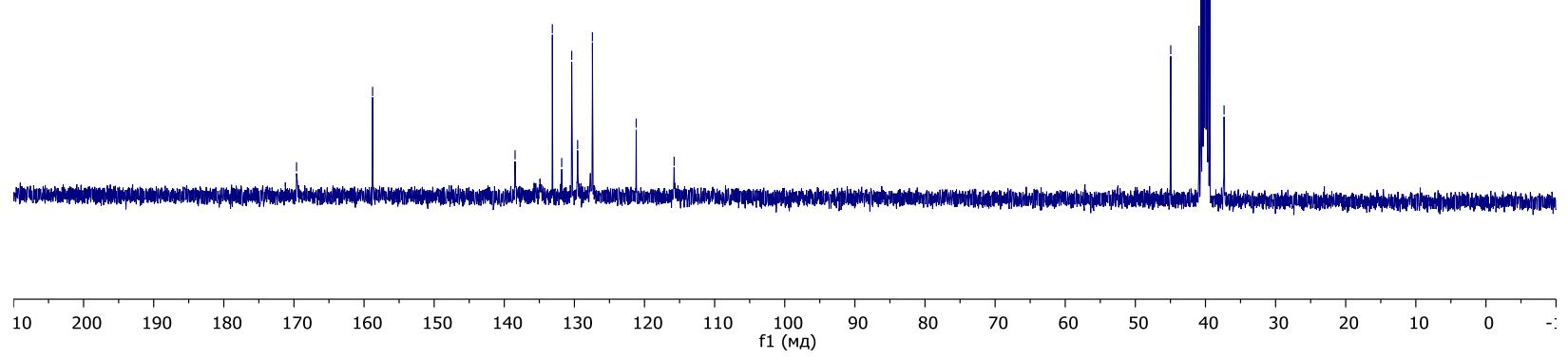

S56 
${ }^{1} \mathrm{H}\left(400 \mathrm{MHz}\right.$; in DMSO- $\left.d_{6}\right)$ and ${ }^{13} \mathrm{C}\left\{{ }^{1} \mathrm{H}\right\}\left(126 \mathrm{MHz}\right.$; in DMSO- $\left.d_{6}\right)$ NMR spectra of compound $12 \mathrm{~b}$

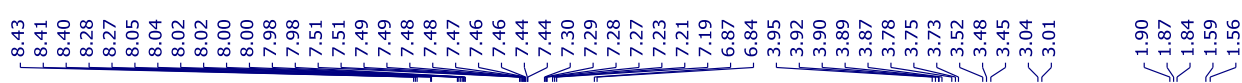<smiles>O=C(NCCCNc1ccc([N+](=O)[O-])cc1Sc1ccccc1)c1ccccc1</smiles>
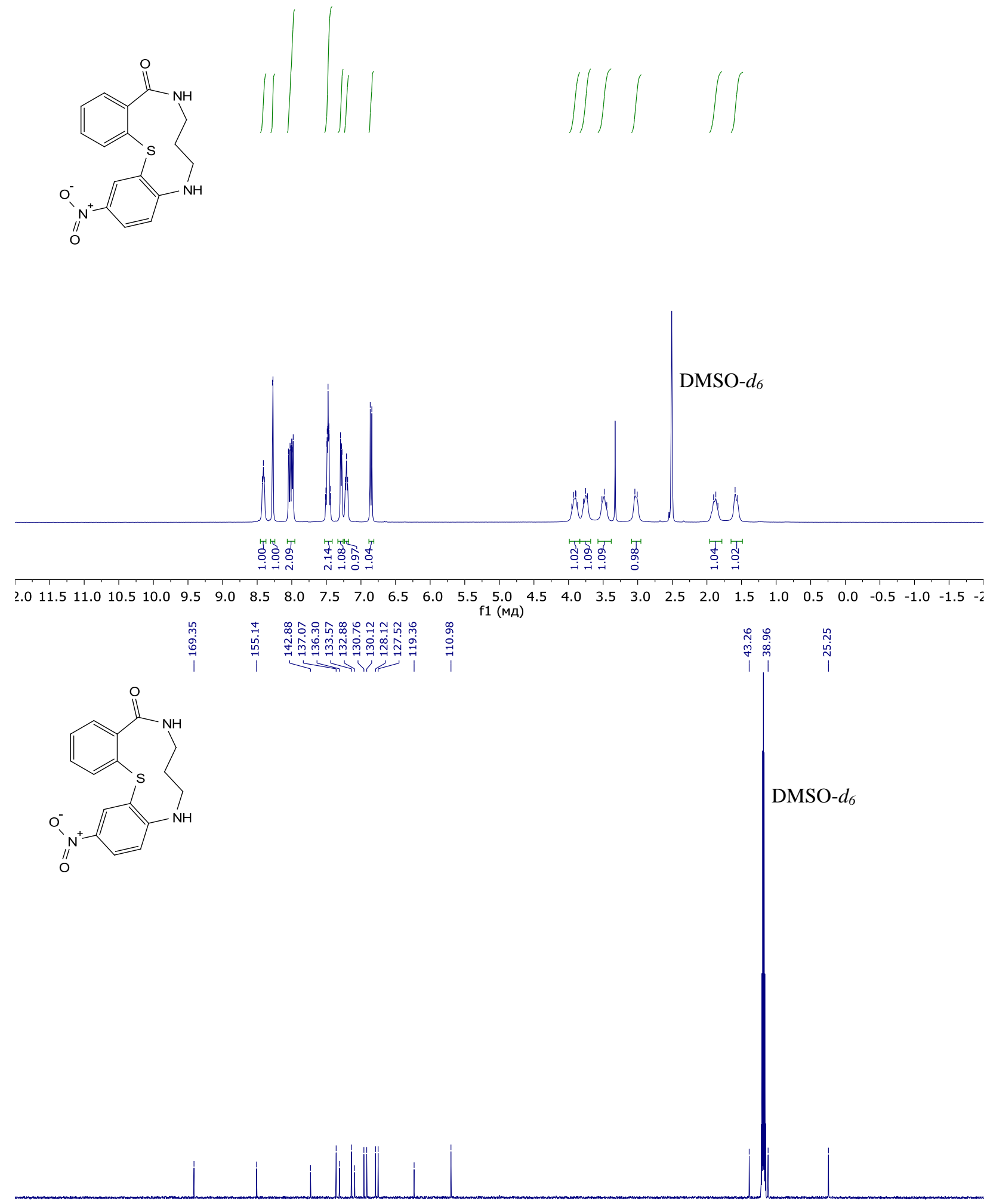

$\begin{array}{llllllllllll}10 & 200 & 190 & 180 & 170 & 160 & 150 & 140 & 130 & 120 & 110 & \begin{array}{l}100 \\ \mathrm{f} 1(\mathrm{ML})\end{array}\end{array}$ 
${ }^{1} \mathrm{H}\left(400 \mathrm{MHz}\right.$; in $\left.\mathrm{CDCl}_{3}\right)$ and ${ }^{13} \mathrm{C}\left\{{ }^{1} \mathrm{H}\right\}\left(126 \mathrm{MHz}\right.$; in DMSO-d $\left.{ }_{6}\right)$ NMR spectra of compound $12 \mathrm{c}$

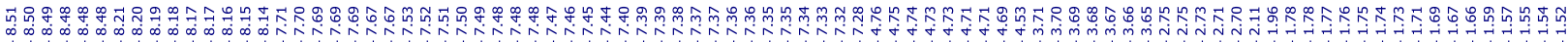<smiles>O=C1NCCCCNc2ccc([N+](=O)[O-])cc2Sc2ccccc21</smiles>
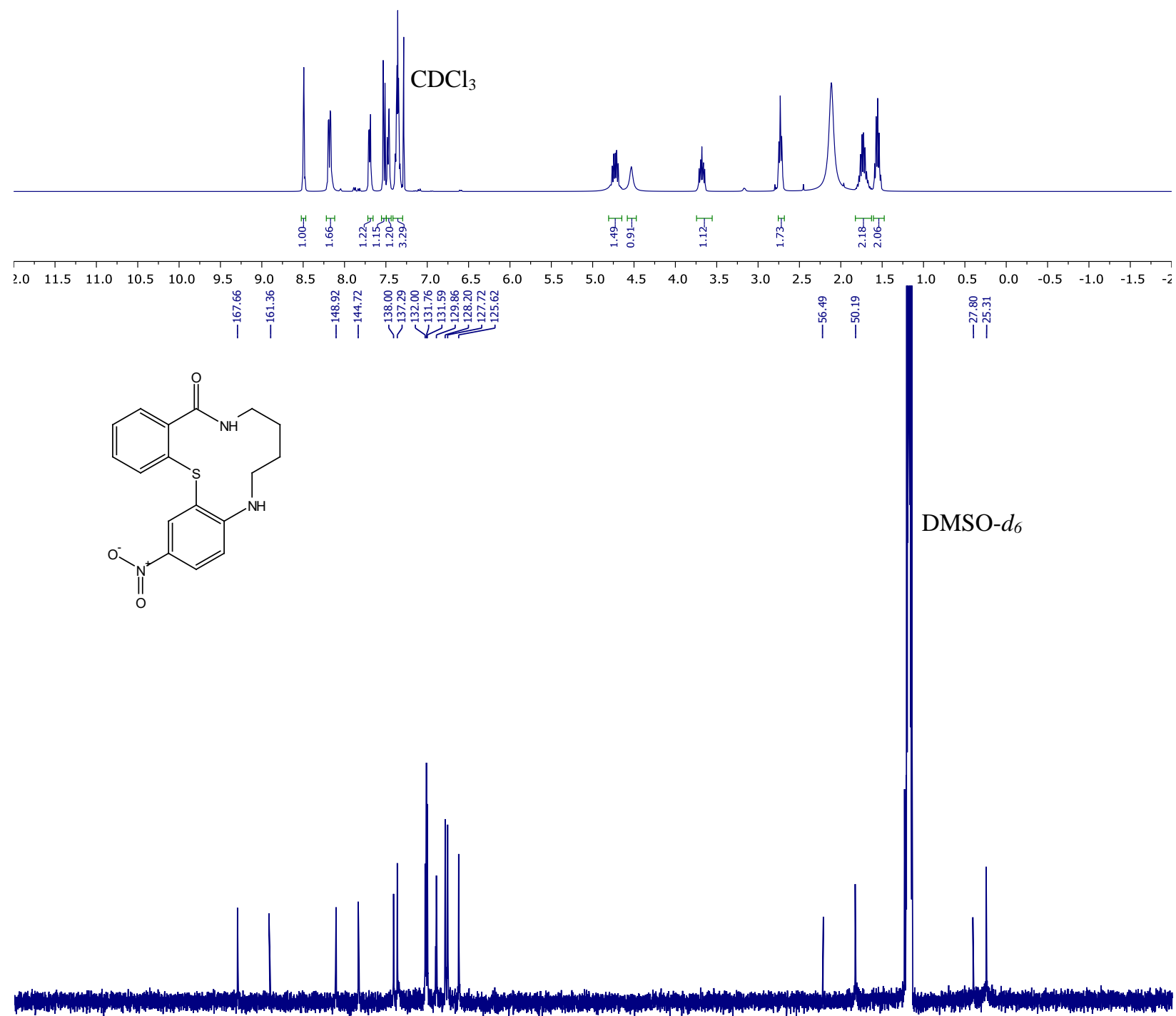

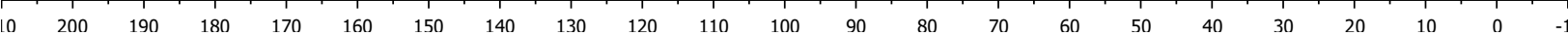


${ }^{1} \mathrm{H}$ (400 MHz; in DMSO- $\left.d_{6}\right)$ and ${ }^{13} \mathrm{C}\left\{{ }^{1} \mathrm{H}\right\}\left(126 \mathrm{MHz}\right.$; in DMSO- $\left.d_{6}\right)$ NMR spectra of compound $12 \mathrm{e}$

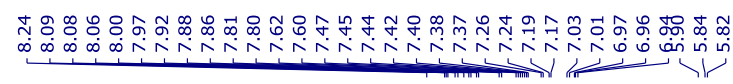
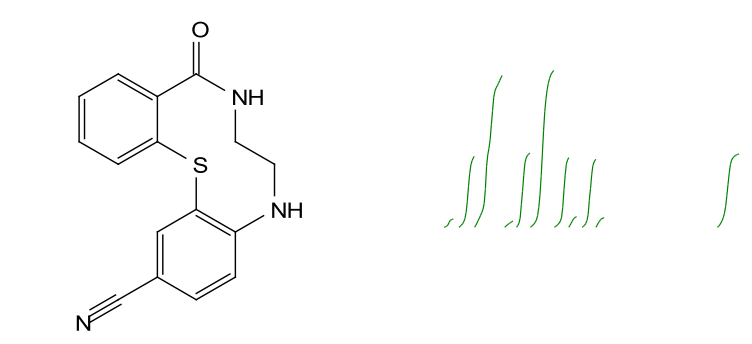

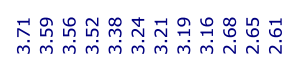

DMSO- $d_{6}$

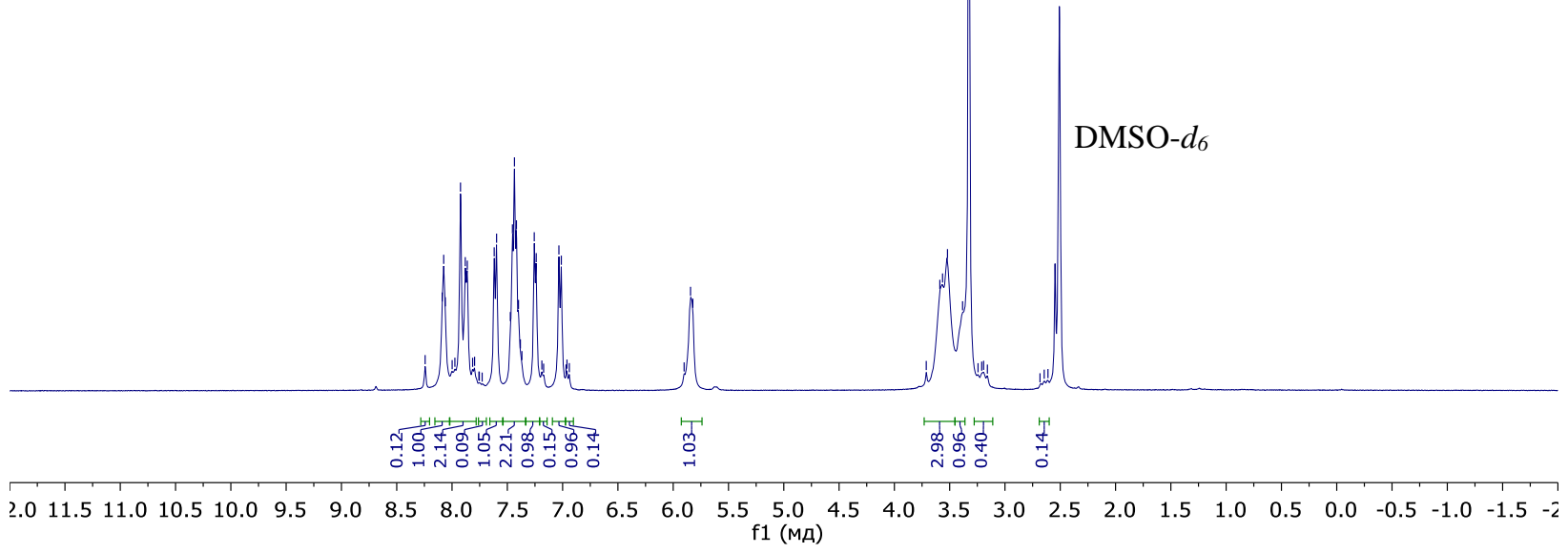

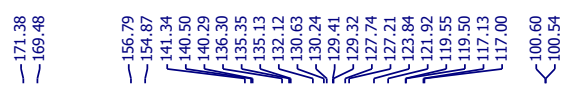
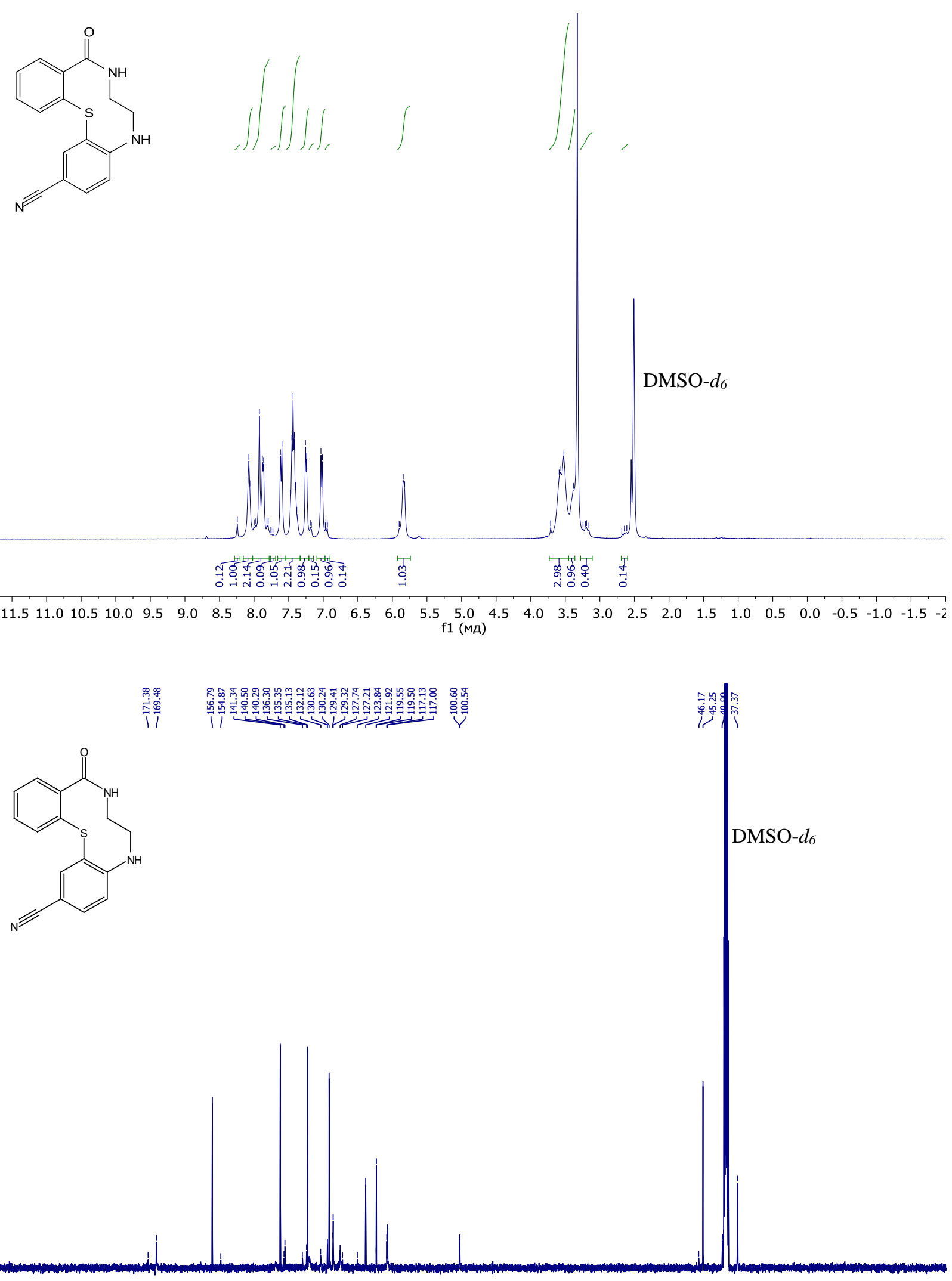

$\begin{array}{lllllllllllllllllllllllllllll}2.0 & 11.5 & 11.0 & 10.5 & 10.0 & 9.5 & 9.0 & 8.5 & 8.0 & 7.5 & 7.0 & 6.5 & 6.0 & 5.5 & 5.0 & 4.5 & 4.0 & 3.5 & 3.0 & 2.5 & 2.0 & 1.5 & 1.0 & 0.5 & 0.0 & -0.5 & -1.0 & -1.5 & -2\end{array}$

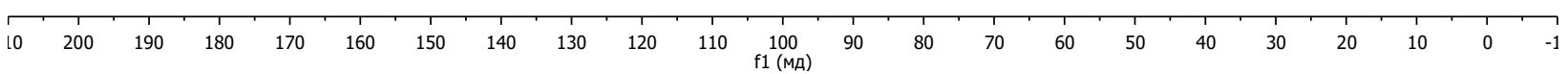

S59 
${ }^{1} \mathrm{H}\left(400 \mathrm{MHz}\right.$; in DMSO- $\left.d_{6}\right)$ and ${ }^{13} \mathrm{C}\left\{{ }^{1} \mathrm{H}\right\}\left(101 \mathrm{MHz}\right.$; in DMSO- $\left.d_{6}\right)$ NMR spectra of compound $12 \mathrm{f}$

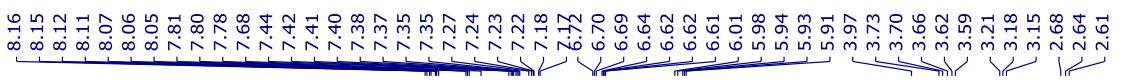
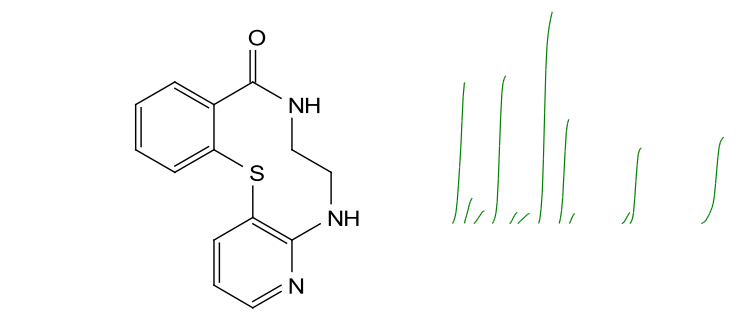

DMSO- $d_{6}$
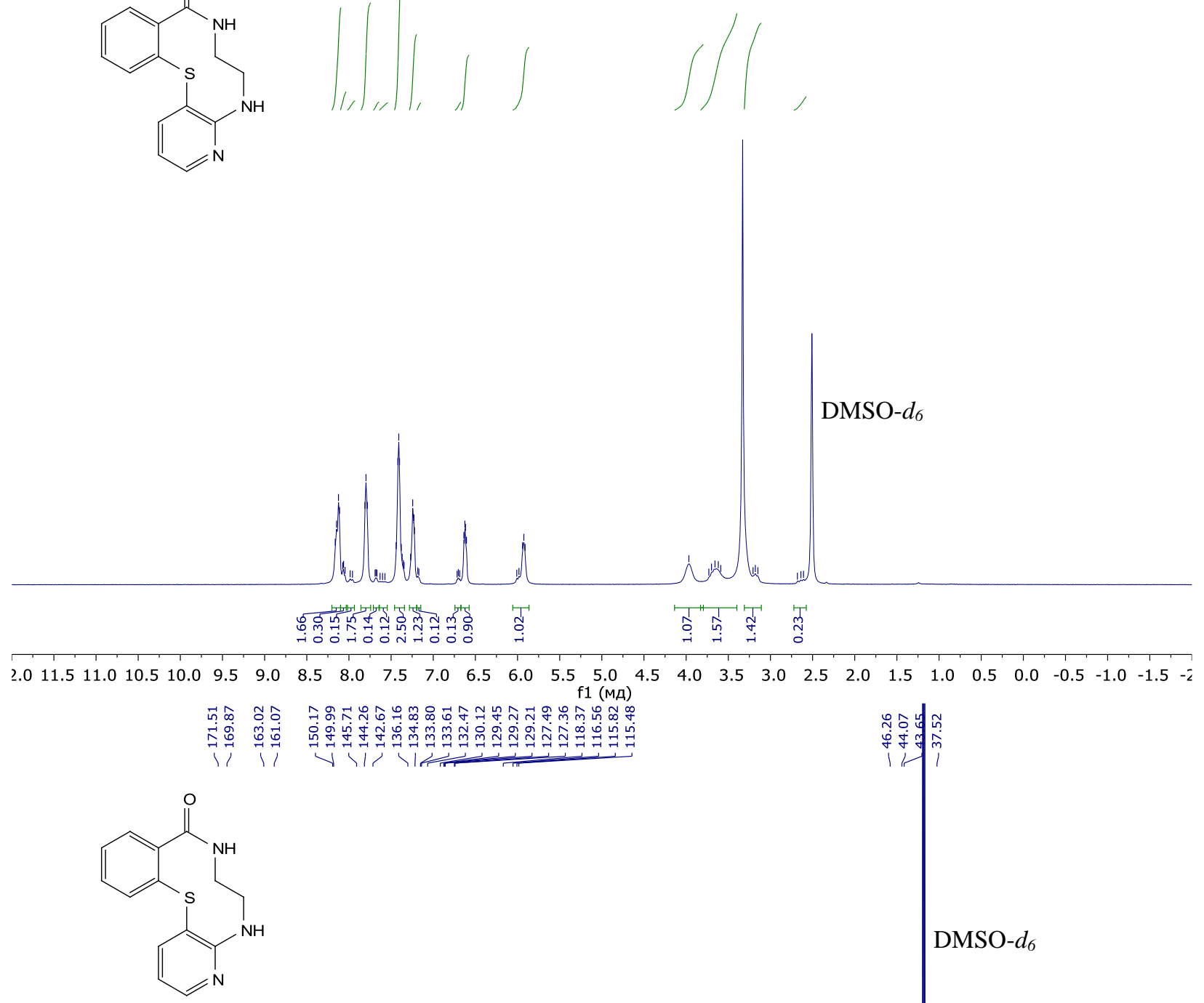

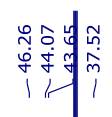

DMSO- $d_{6}$
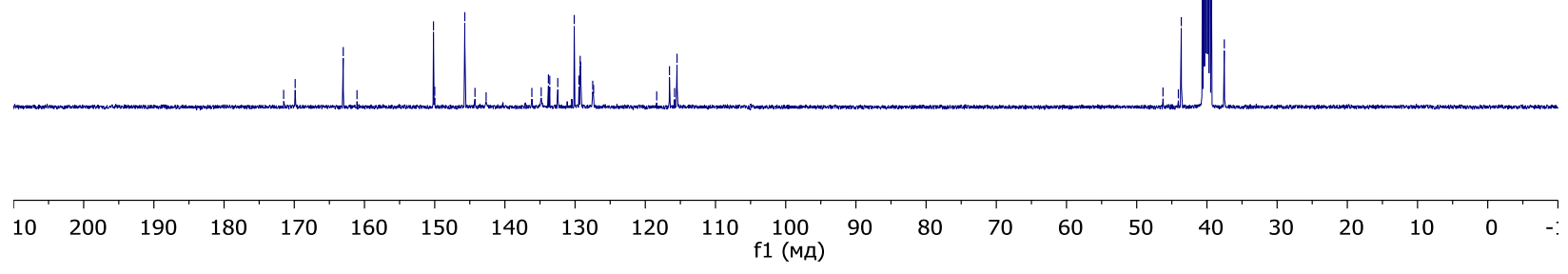

S60 
${ }^{1} \mathrm{H}\left(400 \mathrm{MHz}\right.$; in DMSO- $\left.d_{6}\right)$ and ${ }^{13} \mathrm{C}\left\{{ }^{1} \mathrm{H}\right\}\left(101 \mathrm{MHz}\right.$; in DMSO- $\left.d_{6}\right)$ NMR spectra of compound $12 \mathrm{~g}$

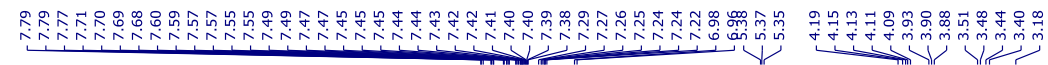

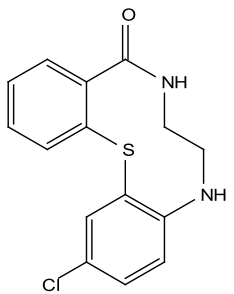

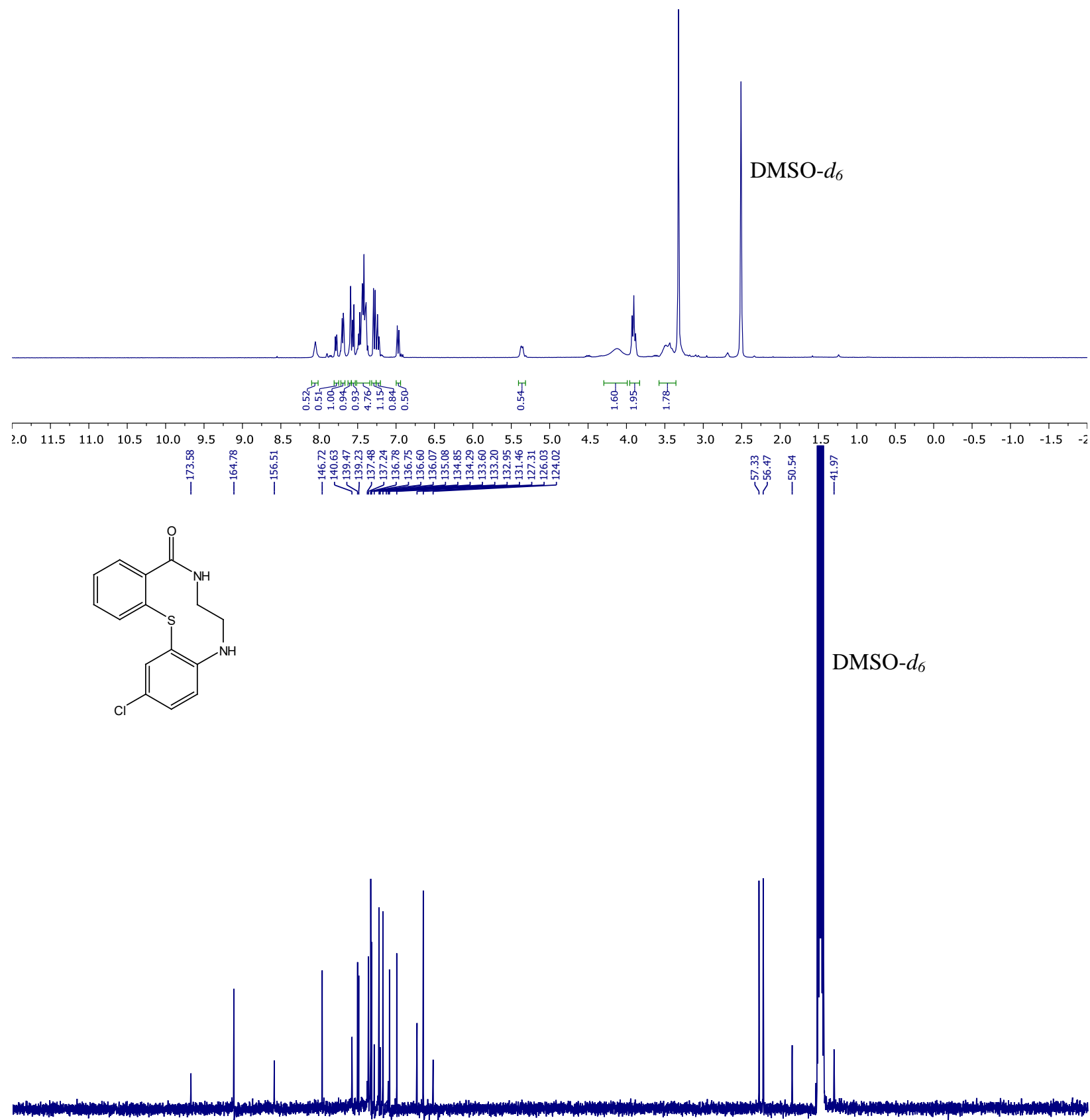

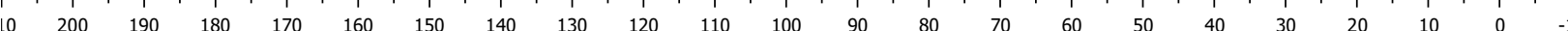


${ }^{1} \mathrm{H}\left(400 \mathrm{MHz}\right.$; in DMSO- $\left.d_{6}\right)$ and ${ }^{13} \mathrm{C}\left\{{ }^{1} \mathrm{H}\right\}\left(101 \mathrm{MHz}\right.$; in DMSO- $\left.d_{6}\right)$ NMR spectra of compound $12 \mathrm{~h}$

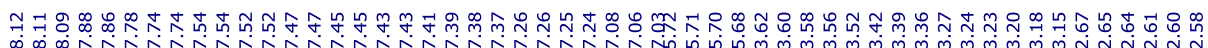<smiles>O=C(NCCNc1ccc(C(F)(F)F)cc1Sc1ccccc1)c1ccccc1</smiles>

\section{$1.11 \| 11$.}
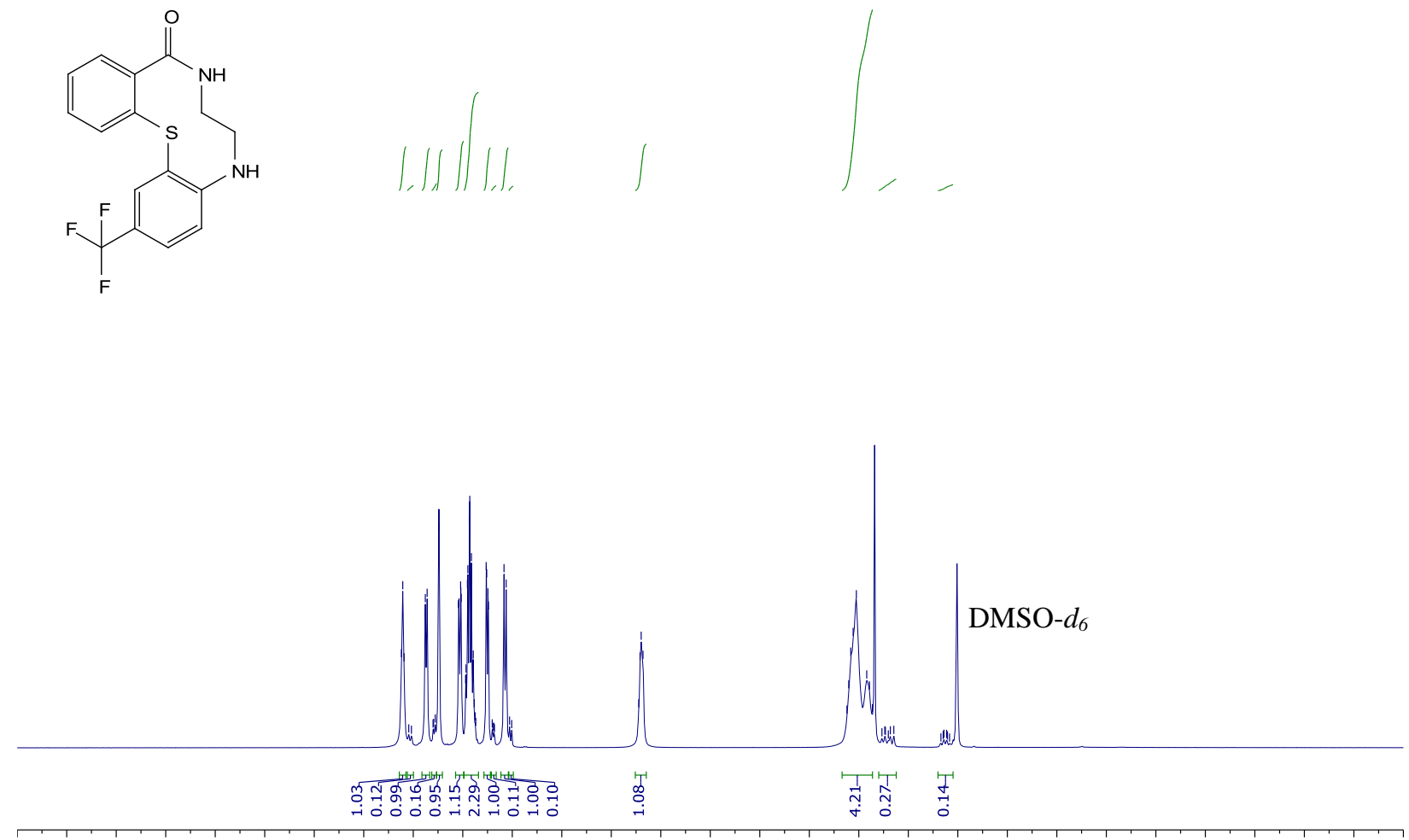
$\begin{array}{llllllllllllllllllllllllllllllllll}2.0 & 11.5 & 11.0 & 10.5 & 10.0 & 9.5 & 9.0 & 8.5 & 8.0 & 7.5 & 7.0 & 6.5 & 6.0 & 5.5 & 5.0 & 4.5 & 4.0 & 3.5 & 3.0 & 2.5 & 2.0 & 1.5 & 1.0 & 0.5 & 0.0 & -0.5 & -1.0 & -1.5 & -2\end{array}$

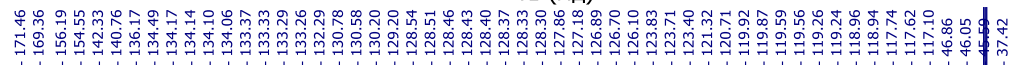<smiles>O=C(NCCNc1ccc(C(F)(F)F)cc1Sc1ccccc1)c1ccccc1</smiles>

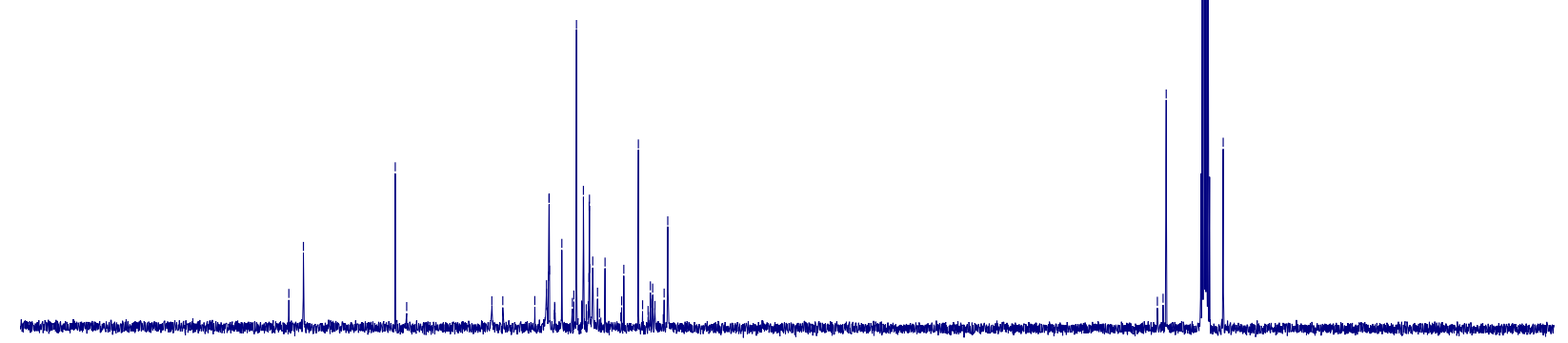

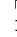

200 
${ }^{1} \mathrm{H}\left(400 \mathrm{MHz}\right.$; in DMSO- $\left.d_{6}\right)$ and ${ }^{13} \mathrm{C}\left\{{ }^{1} \mathrm{H}\right\}\left(101 \mathrm{MHz}\right.$; in DMSO- $\left.d_{6}\right)$ NMR spectra of compound 12i

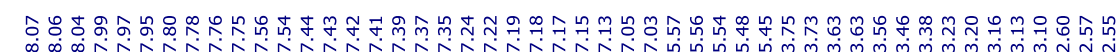<smiles>N#Cc1ccc(Sc2ccccc2C(=O)NCCNc2ccccc2)cc1</smiles><smiles></smiles>
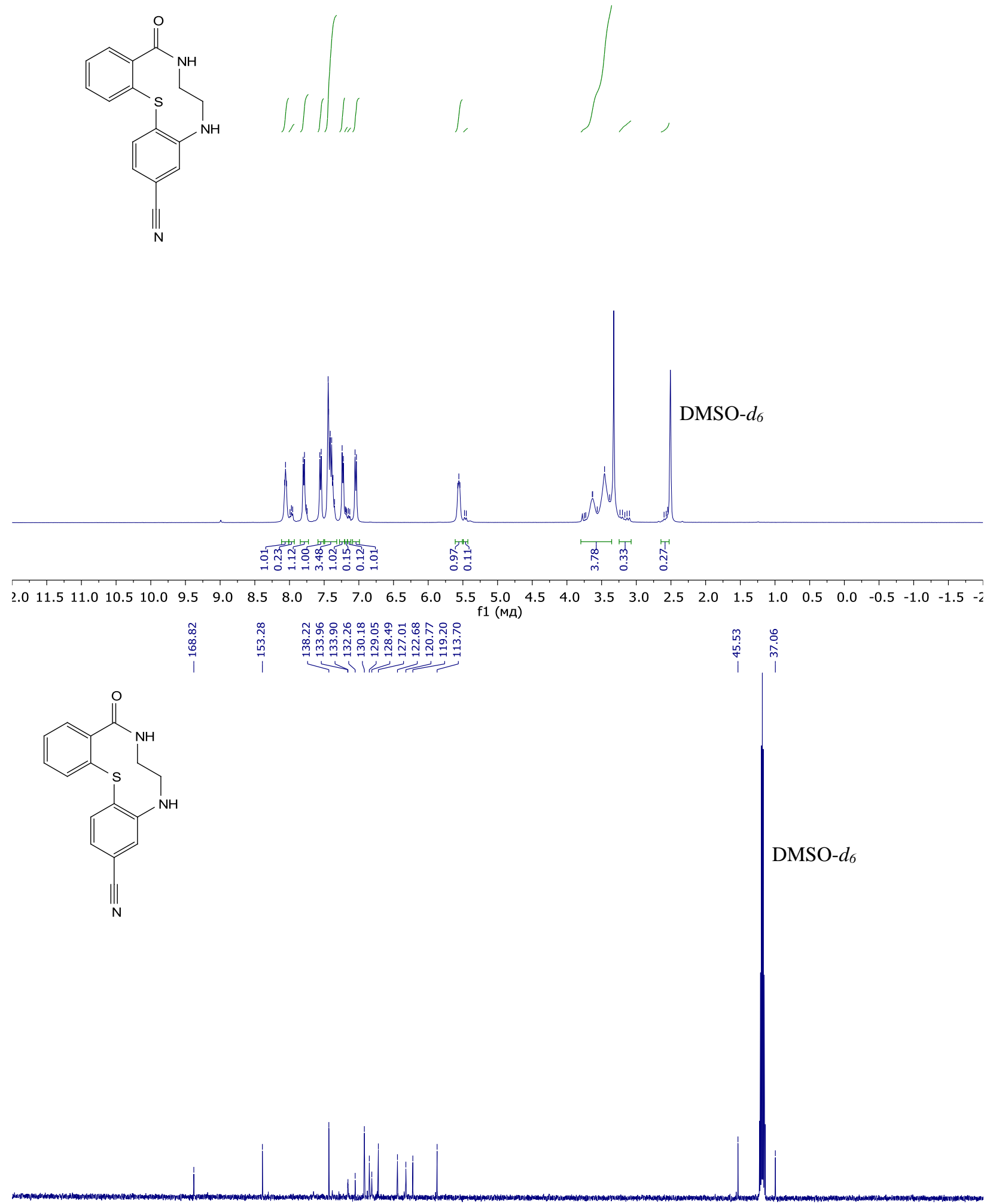

10

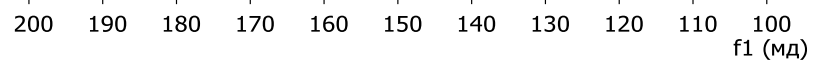

$90 \quad 80 \quad 70 \quad 60 \quad 50 \quad 40$

3020

10 
${ }^{1} \mathrm{H}$ (400 MHz; in DMSO- $\left.d_{6}\right)$ and ${ }^{13} \mathrm{C}\left\{{ }^{1} \mathrm{H}\right\}\left(101 \mathrm{MHz}\right.$; in DMSO- $\left.d_{6}\right)$ NMR spectra of compound 12j

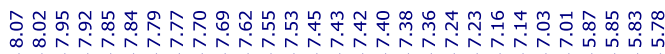<smiles>Cc1cc(S(=O)(=O)N(C)C)ccc1NCCNC(=O)c1ccccc1Sc1ccccc1</smiles>

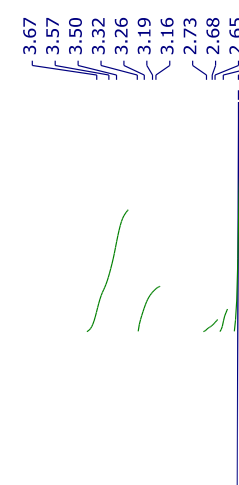

(1.5)

DMSO- $d_{6}$ 
${ }^{1} \mathrm{H}\left(400 \mathrm{MHz}\right.$; in DMSO- $\left.d_{6}\right)$ and ${ }^{13} \mathrm{C}\left\{{ }^{1} \mathrm{H}\right\}\left(101 \mathrm{MHz}\right.$; in DMSO- $\left.d_{6}\right)$ NMR spectra of compound 12k

F
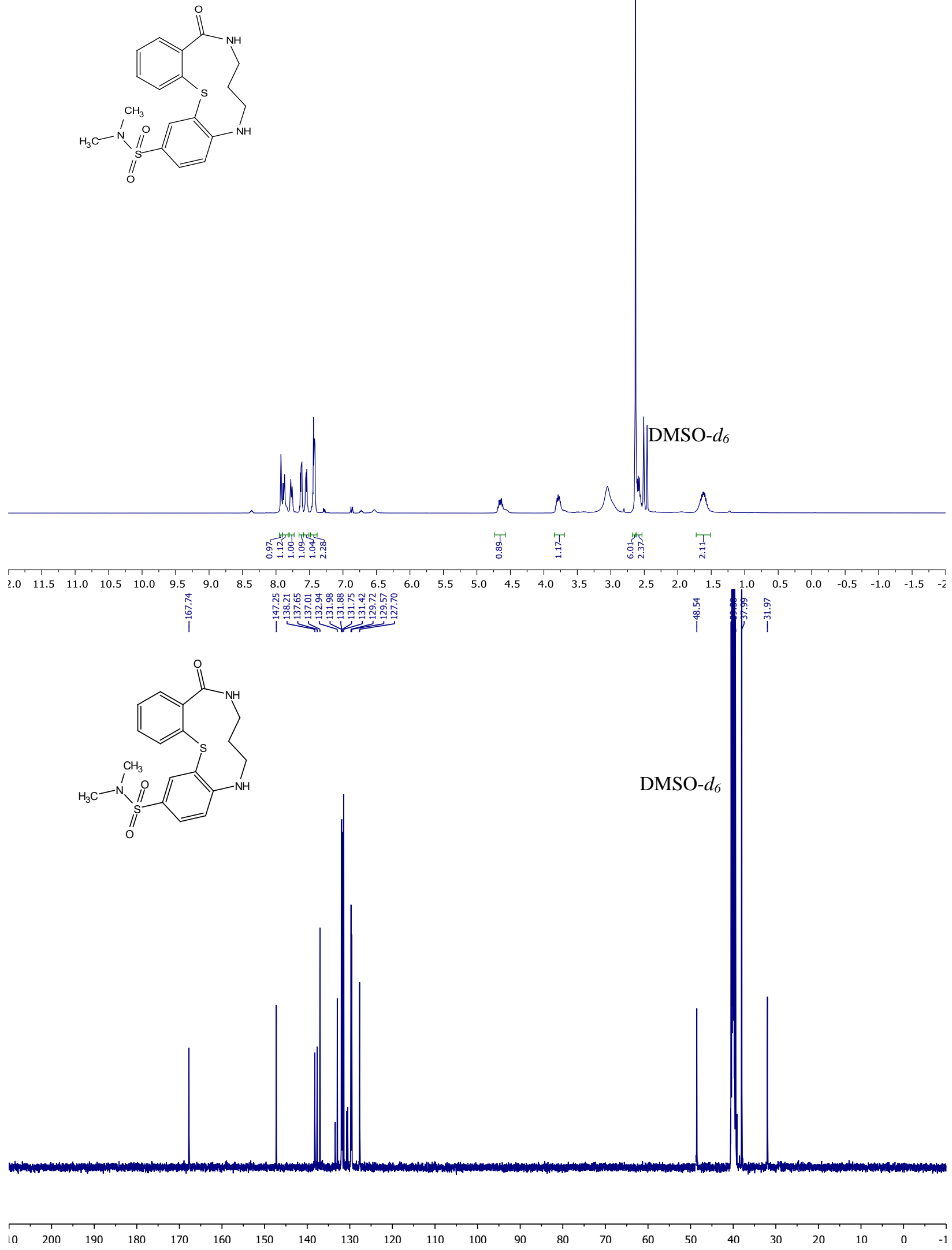

S65 
${ }^{1} \mathrm{H}\left(400 \mathrm{MHz}\right.$; in DMSO- $\left.d_{6}\right)$ and ${ }^{13} \mathrm{C}\left\{{ }^{1} \mathrm{H}\right\}\left(101 \mathrm{MHz}\right.$; in DMSO- $\left.d_{6}\right)$ NMR spectra of compound $12 \mathrm{I}$

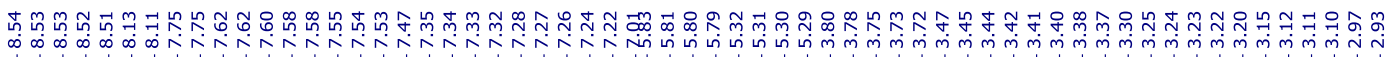<smiles>O=C(NCCNc1ccc(C(F)(F)F)cc1Sc1ncccc1C(=O)O)c1cccnc1</smiles>

DMSO- $d_{6}$

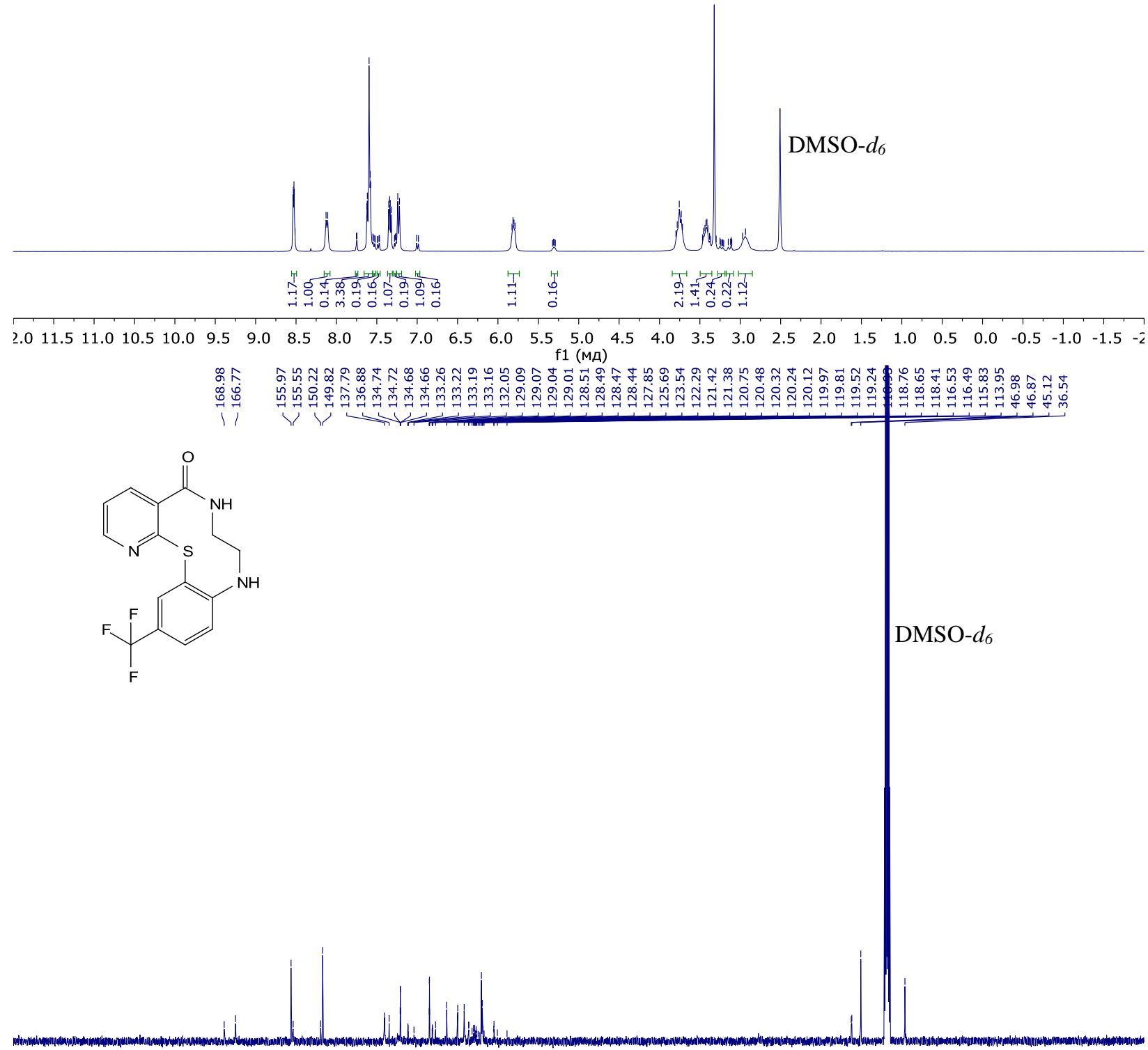

$\begin{array}{lllllllllllllllllllllll}10 & 200 & 190 & 180 & 170 & 160 & 150 & 140 & 130 & 120 & 110 & 100 & 90 & 80 & 70 & 60 & 50 & 40 & 30 & 20 & 10 & 0 & -\end{array}$ 
${ }^{1} \mathrm{H}\left(400 \mathrm{MHz}\right.$; in DMSO- $\left.d_{6}\right)$ and ${ }^{13} \mathrm{C}\left\{{ }^{1} \mathrm{H}\right\}\left(101 \mathrm{MHz}\right.$; in DMSO- $\left.d_{6}\right)$ NMR spectra of compound 12m

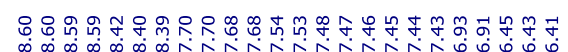

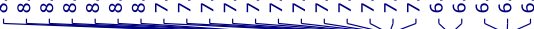

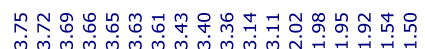<smiles>O=C(NCCCNc1ccc(C(F)(F)F)cc1Sc1ncccc1C(=O)O)c1ccccc1</smiles>
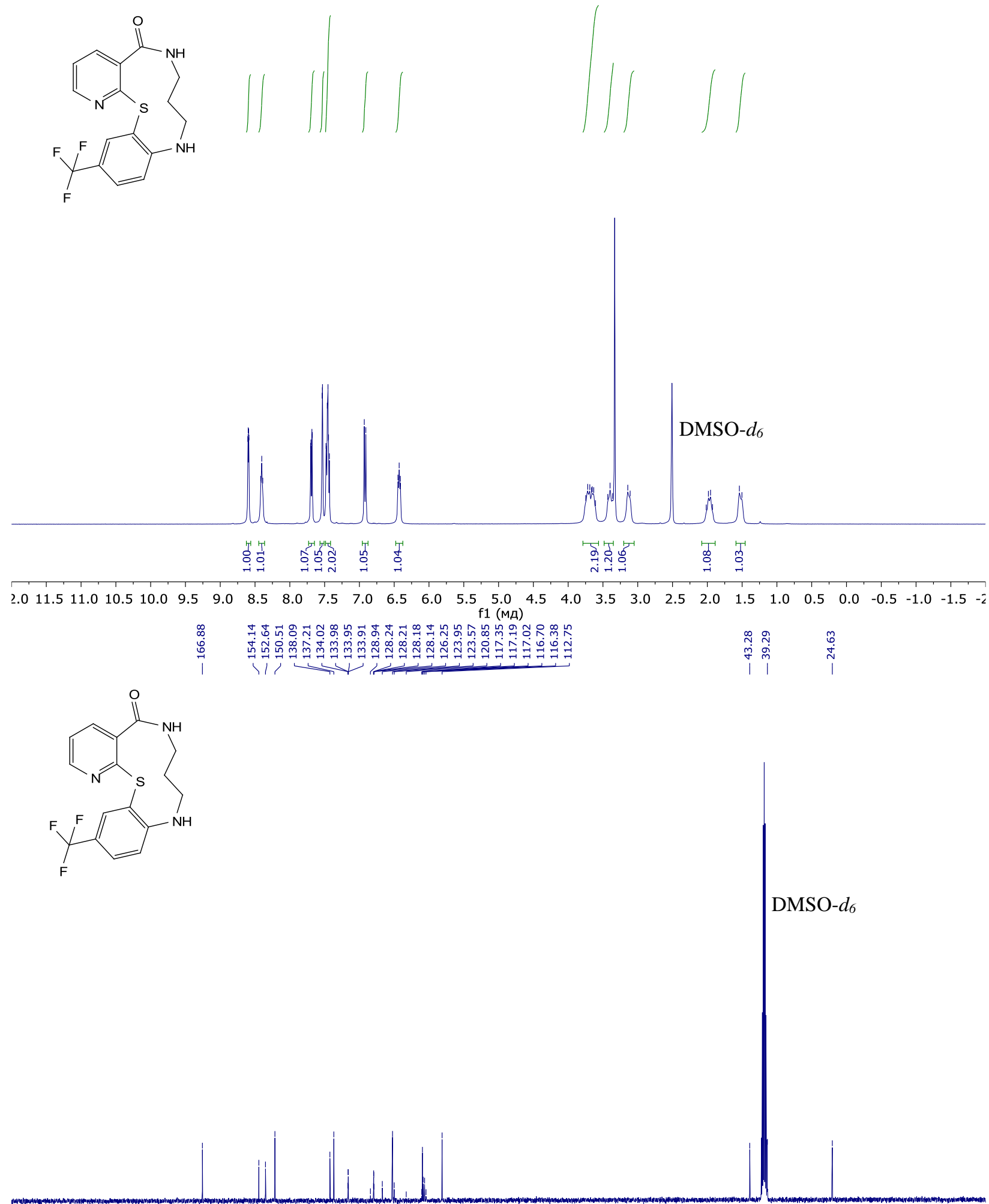

10

$\begin{array}{lllllllllll}200 & 190 & 180 & 170 & 160 & 150 & 140 & 130 & 120 & 110 & \begin{array}{c}100 \\ \mathrm{f} 1(\mathrm{MA})\end{array}\end{array}$ $90 \quad 80 \quad 70 \quad 60 \quad 50 \quad 40$ 
${ }^{1} \mathrm{H}$ (400 MHz; in DMSO- $\left.d_{6}\right)$ and ${ }^{13} \mathrm{C}\left\{{ }^{1} \mathrm{H}\right\}\left(126 \mathrm{MHz}\right.$; in DMSO- $\left.d_{6}\right)$ NMR spectra of compound 12n

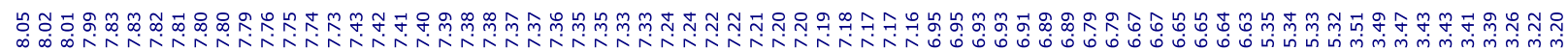<smiles>O=C(NCCNc1ccccc1Sc1ccccc1)c1ccccc1</smiles>
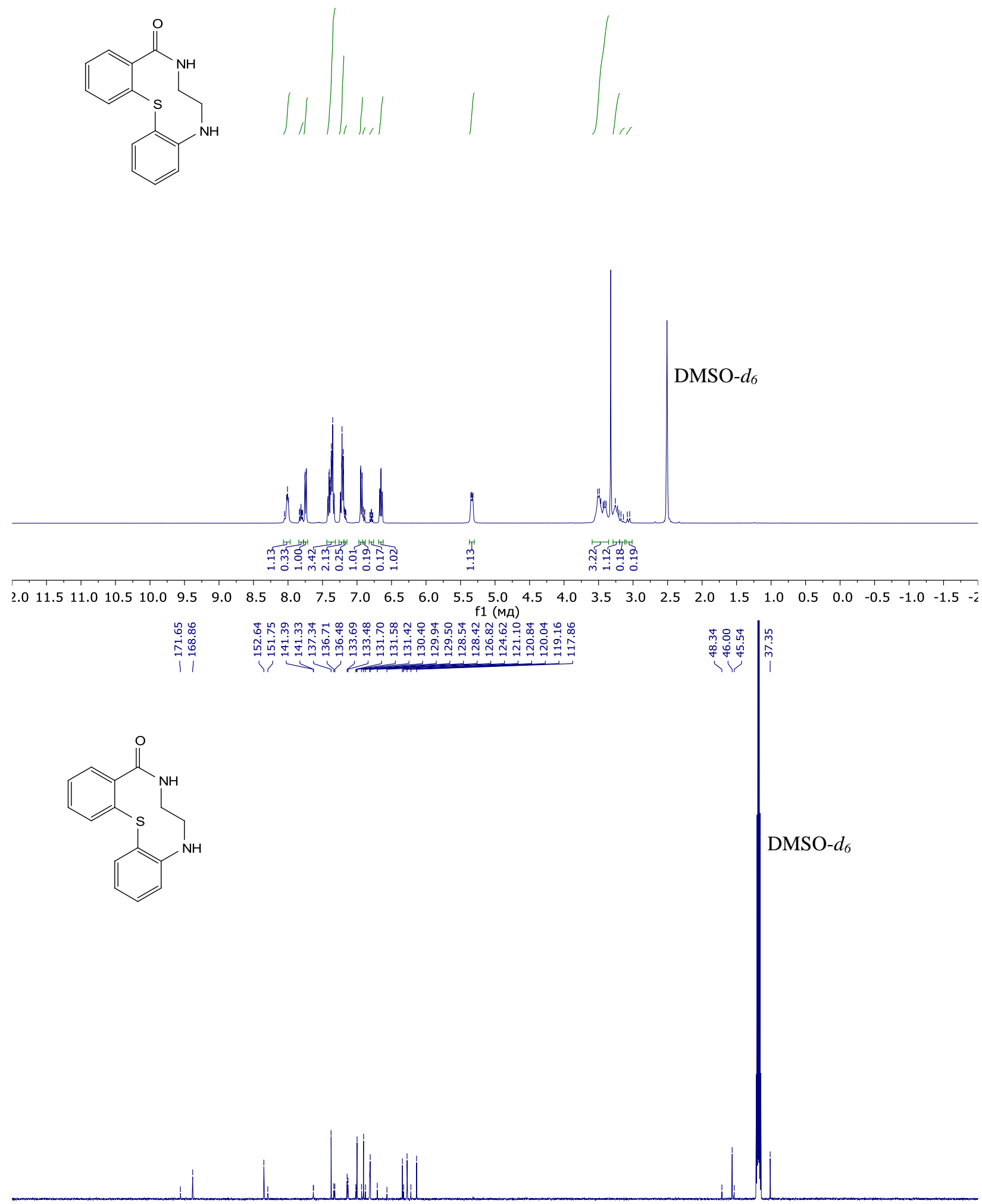

10200

$\begin{array}{lllllllllll}100 & 190 & 180 & 170 & 160 & 150 & 140 & 130 & 120 & 110 & 100 \\ \mathrm{f} 1(\mathrm{MA})\end{array}$

$\begin{array}{llll}90 & 80 & 70 & 60\end{array}$

$50 \quad 40$

$30 \quad 20$ 
<smiles></smiles>

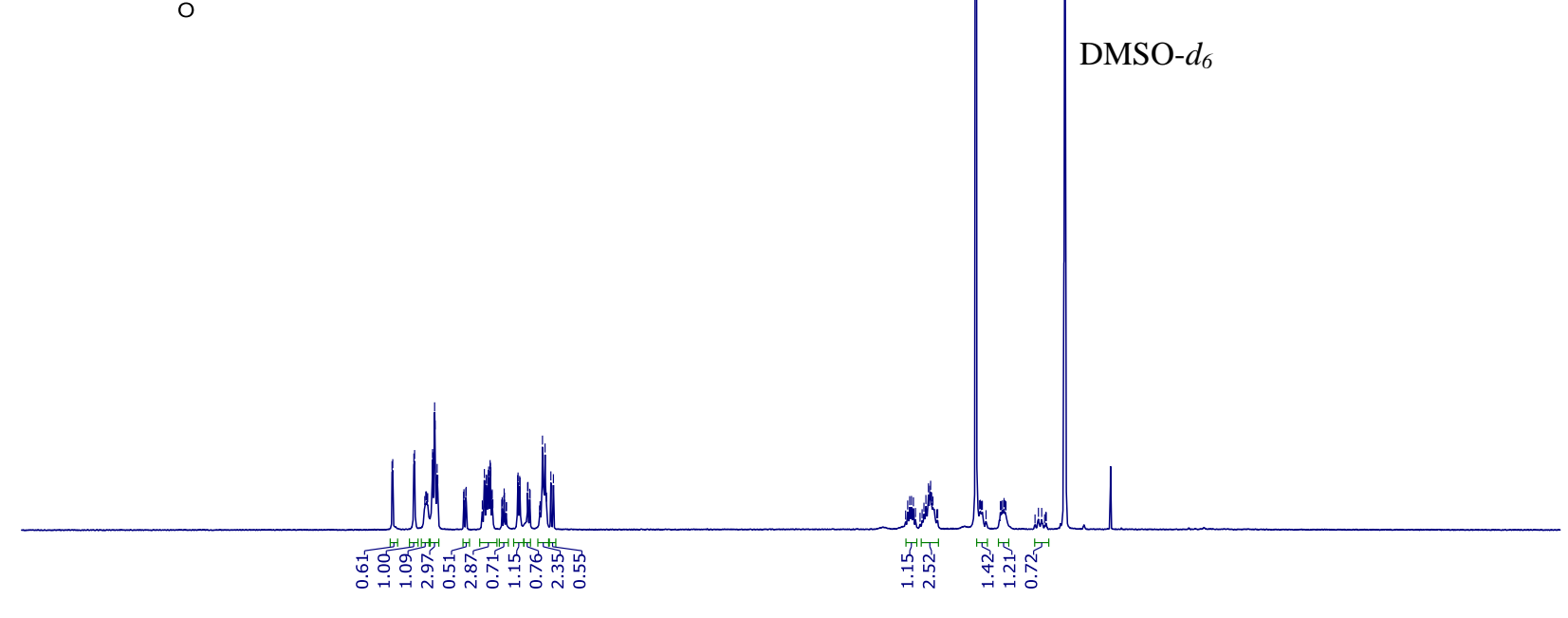

$\begin{array}{lllllllllllllllllllllllllllllllllllllllllllll}11.5 & 11.0 & 10.5 & 10.0 & 9.5 & 9.0 & 8.5 & 8.0 & 7.5 & 7.0 & 6.5 & 6.0 & 5.5 & 5.0 & 4.5 & 4.0 & 3.5 & 3.0 & 2.5 & 2.0 & 1.5 & 1.0 & 0.5 & 0.0 & -0.5 & -1.0 & -1.5 & -2\end{array}$

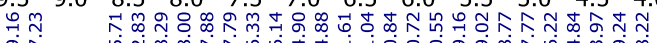
ôt<smiles>Cc1ccccc1S(=O)(=O)c1cc([N+](=O)[O-])ccc1NCCNC(=O)c1ccccc1</smiles>

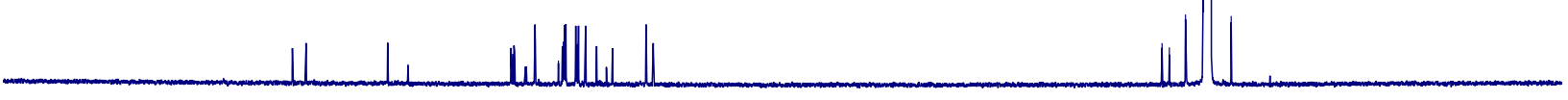

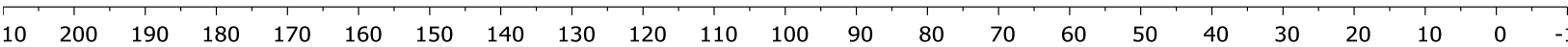


${ }^{1} \mathrm{H}\left(400 \mathrm{MHz}\right.$; in DMSO- $\left.d_{6}\right)$ and ${ }^{13} \mathrm{C}\left\{{ }^{1} \mathrm{H}\right\}\left(101 \mathrm{MHz}\right.$; in DMSO- $\left.d_{6}\right)$ NMR spectra of compound 13b

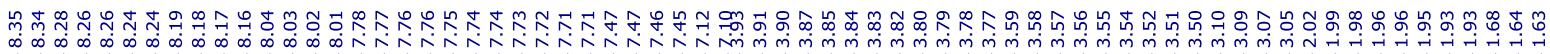<smiles>O=C1NCCCNc2ccc([N+](=O)[O-])cc2S(=O)(=O)c2ccccc21</smiles>
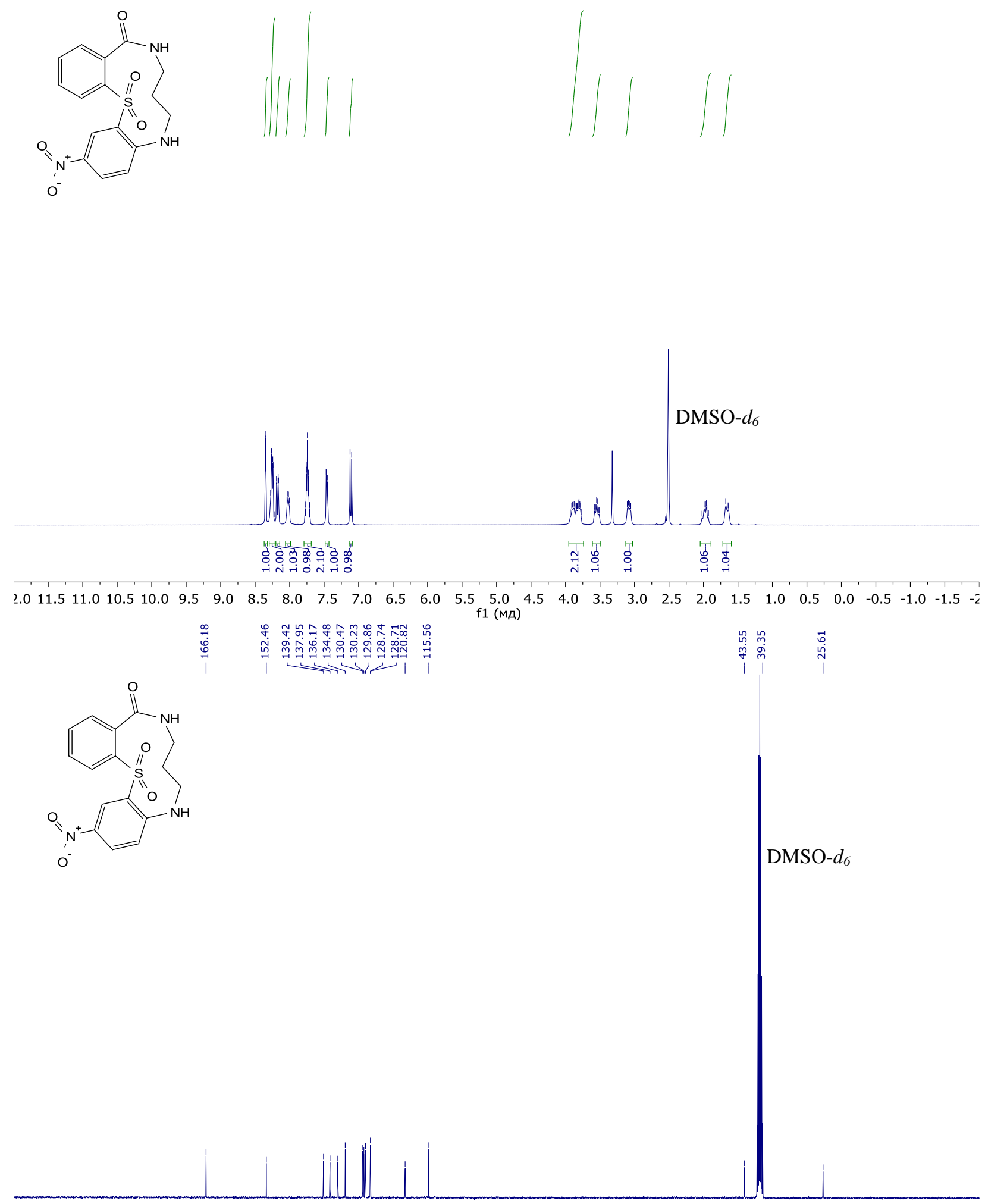

10

$\begin{array}{lllllllllll}200 & 190 & 180 & 170 & 160 & 150 & 140 & 130 & 120 & 110 & \begin{array}{l}100 \\ \mathrm{f} 1(\mathrm{M})\end{array}\end{array}$ 
${ }^{1} \mathrm{H}\left(400 \mathrm{MHz}\right.$; in DMSO- $\left.d_{6}\right)$ and ${ }^{13} \mathrm{C}\left\{{ }^{1} \mathrm{H}\right\}\left(101 \mathrm{MHz}\right.$; in DMSO- $\left.d_{6}\right)$ NMR spectra of compound $13 \mathrm{e}$

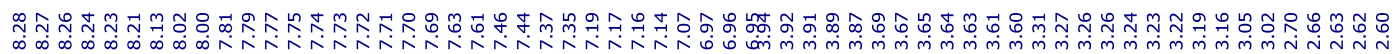<smiles>Cc1cc(C#N)ccc1NCCNC(=O)c1ccccc1S(=O)(=O)[O-]</smiles>
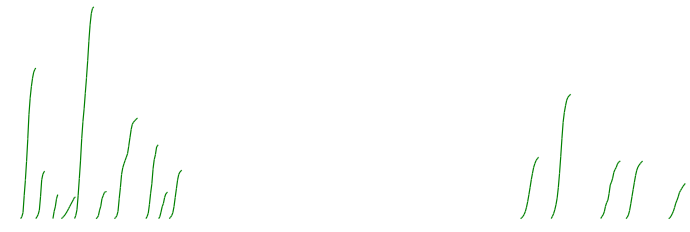

N

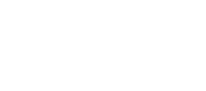

DMSO- $d_{6}$

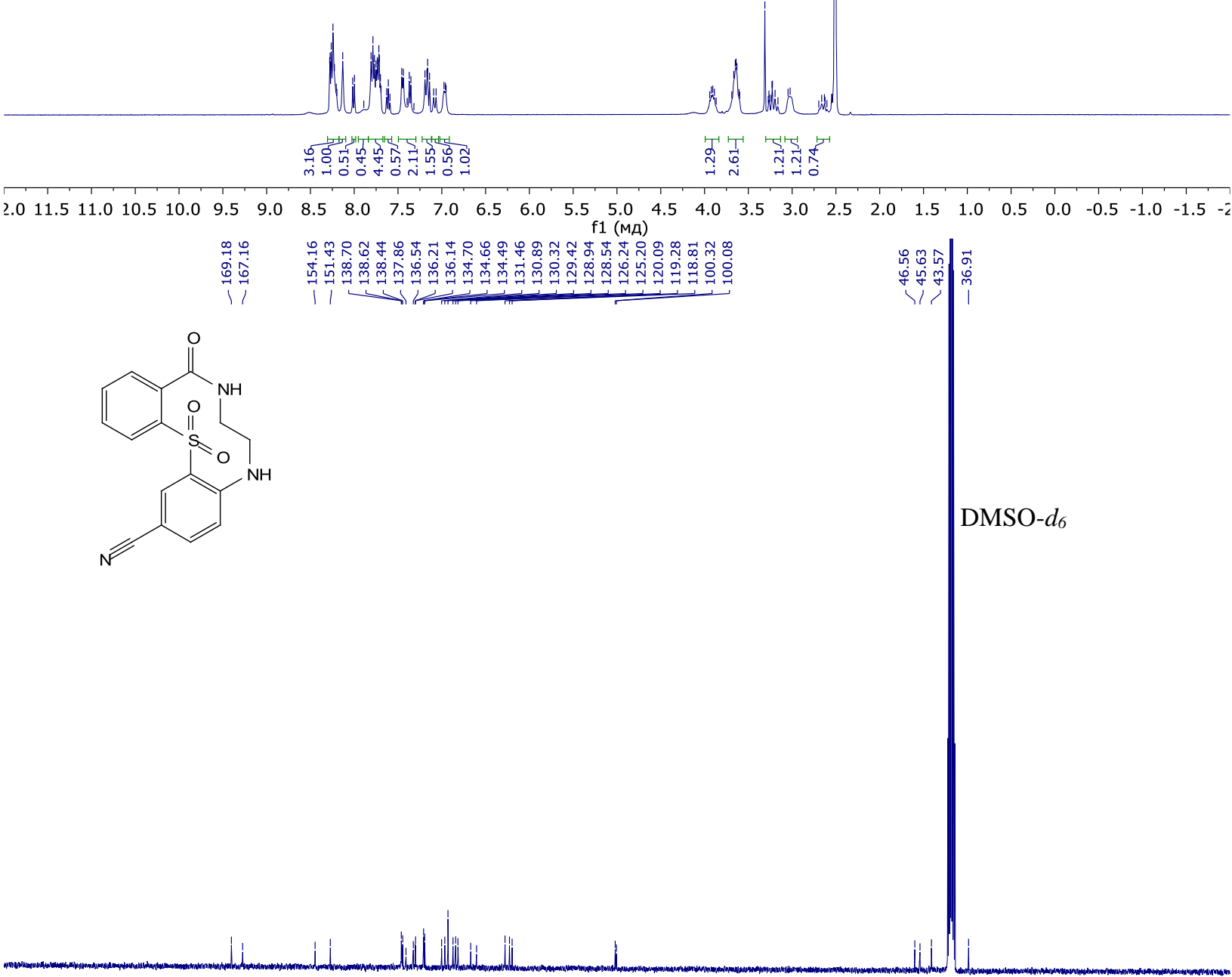

10200

$\begin{array}{llllllllll}190 & 180 & 170 & 160 & 150 & 140 & 130 & 120 & 110 & 100\end{array}$

$\begin{array}{llll}90 & 80 & 70 & 60\end{array}$

$50 \quad 40$

$30 \quad 20$


${ }^{1} \mathrm{H}\left(400 \mathrm{MHz}\right.$; in DMSO- $\left.d_{6}\right)$ and ${ }^{13} \mathrm{C}\left\{{ }^{1} \mathrm{H}\right\}\left(126 \mathrm{MHz}\right.$; in DMSO- $\left.d_{6}\right)$ NMR spectra of compound $13 \mathrm{f}$

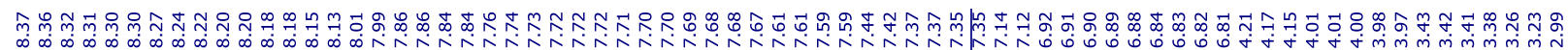<smiles>C#CC(C)CCNc1ncccc1S(=O)(=O)c1ccccc1</smiles>

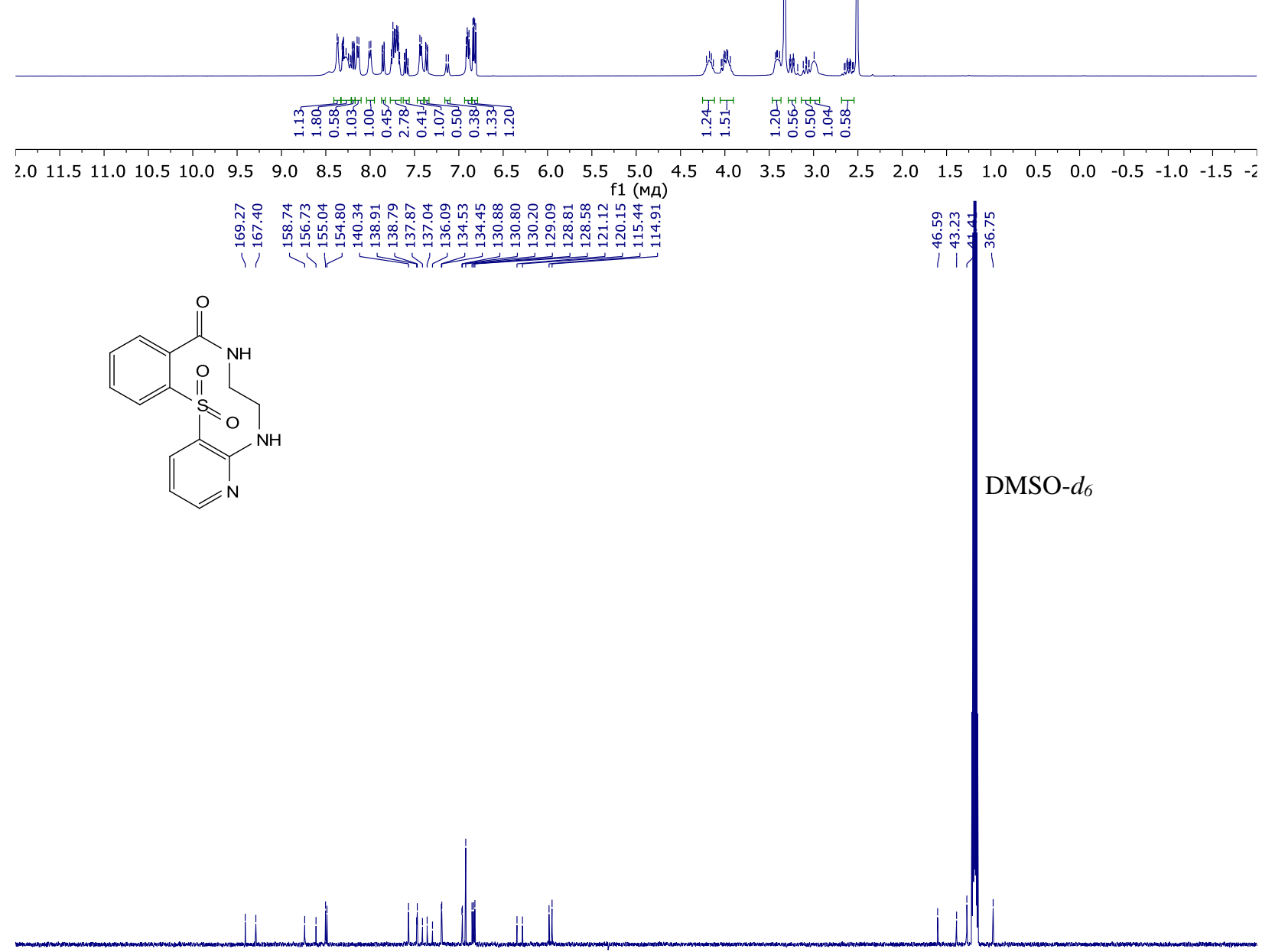

\begin{tabular}{lllllllllllllllllllllll}
\hline 10 & 200 & 190 & 180 & 170 & 160 & 150 & 140 & 130 & 120 & 110 & $\begin{array}{c}100 \\
\mathrm{f} 1(\mathrm{MA})\end{array}$ & 90 & 80 & 70 & 60 & 50 & 40 & 30 & 20 & 10 & 0 & -
\end{tabular} 
${ }^{1} \mathrm{H}$ (400 MHz; in DMSO- $\left.d_{6}\right)$ and ${ }^{13} \mathrm{C}\left\{{ }^{1} \mathrm{H}\right\}\left(101 \mathrm{MHz}\right.$; in DMSO- $\left.d_{6}\right)$ NMR spectra of compound $13 \mathrm{~g}$

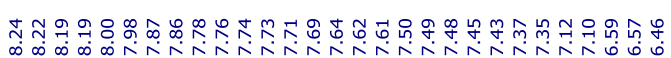

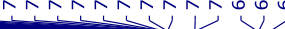

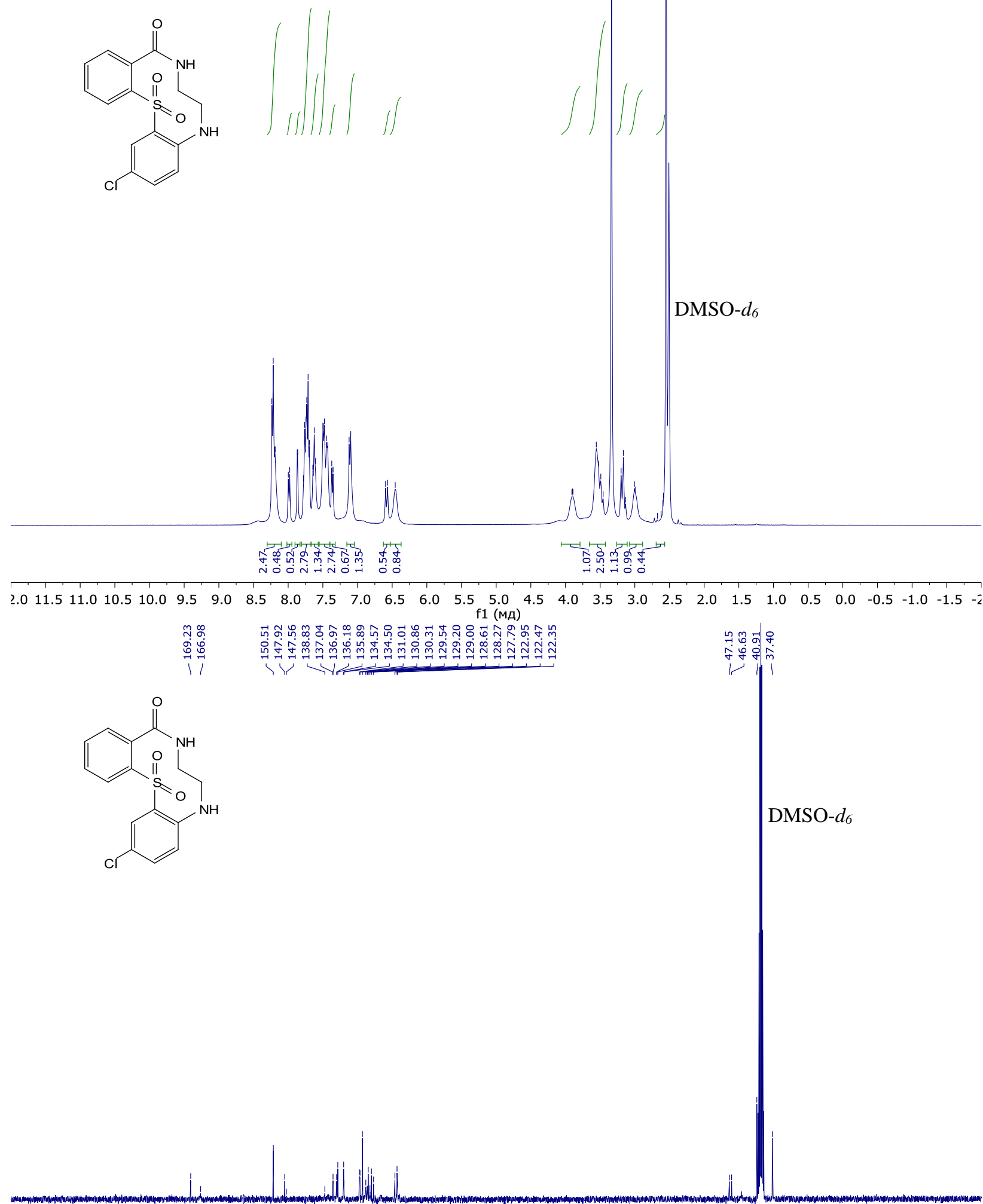

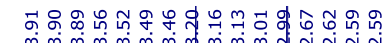

$\begin{array}{llllllllllllllllllllllll}10 & 200 & 190 & 180 & 170 & 160 & 150 & 140 & 130 & 120 & 110 & 100 & 90 & 80 & 70 & 60 & 50 & 40 & 30 & 20 & 10 & 0 & -\end{array}$ 
${ }^{1} \mathrm{H}\left(400 \mathrm{MHz}\right.$; in DMSO- $\left.d_{6}\right)$ and ${ }^{13} \mathrm{C}\left\{{ }^{1} \mathrm{H}\right\}\left(101 \mathrm{MHz}\right.$; in DMSO- $\left.d_{6}\right)$ NMR spectra of compound $13 \mathrm{~h}$

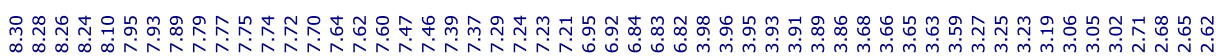<smiles>O=C(NCCNc1ccc(C(F)(F)F)cc1S(=O)(=O)O)c1ccccc1</smiles>
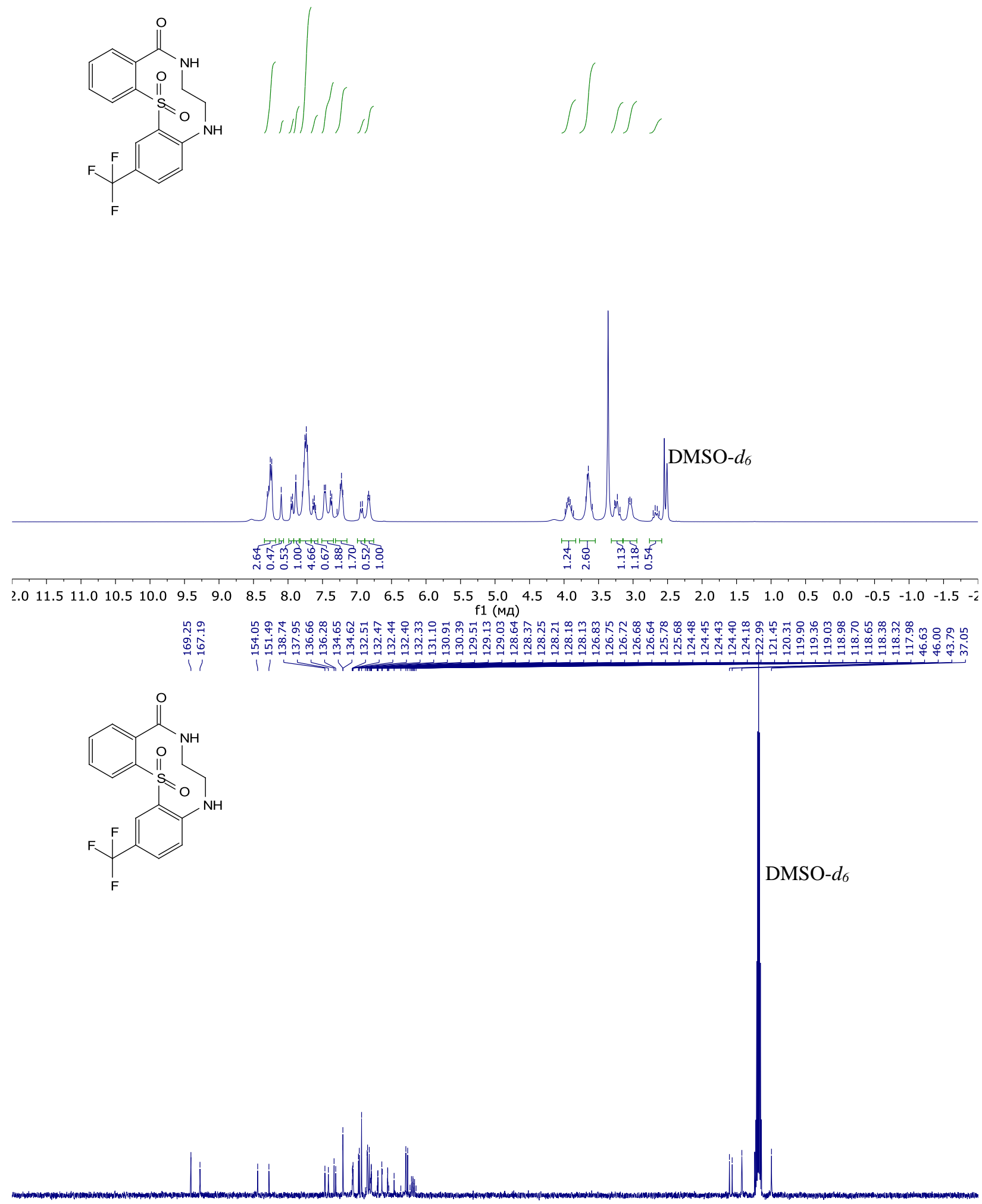

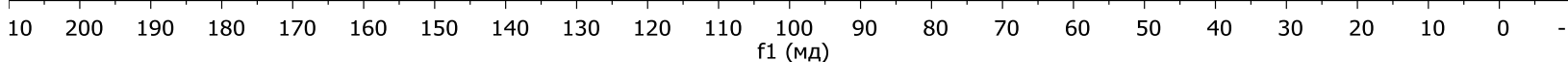


${ }^{1} \mathrm{H}\left(400 \mathrm{MHz}\right.$; in DMSO- $\left.d_{6}\right)$ and ${ }^{13} \mathrm{C}\left\{{ }^{1} \mathrm{H}\right\}\left(126 \mathrm{MHz}\right.$; in DMSO- $\left.d_{6}\right)$ NMR spectra of compound $13 \mathrm{i}$

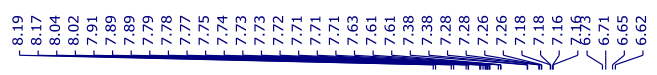

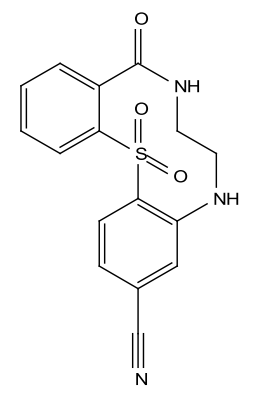

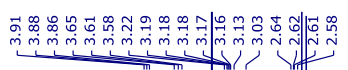

DMSO- $d_{6}$
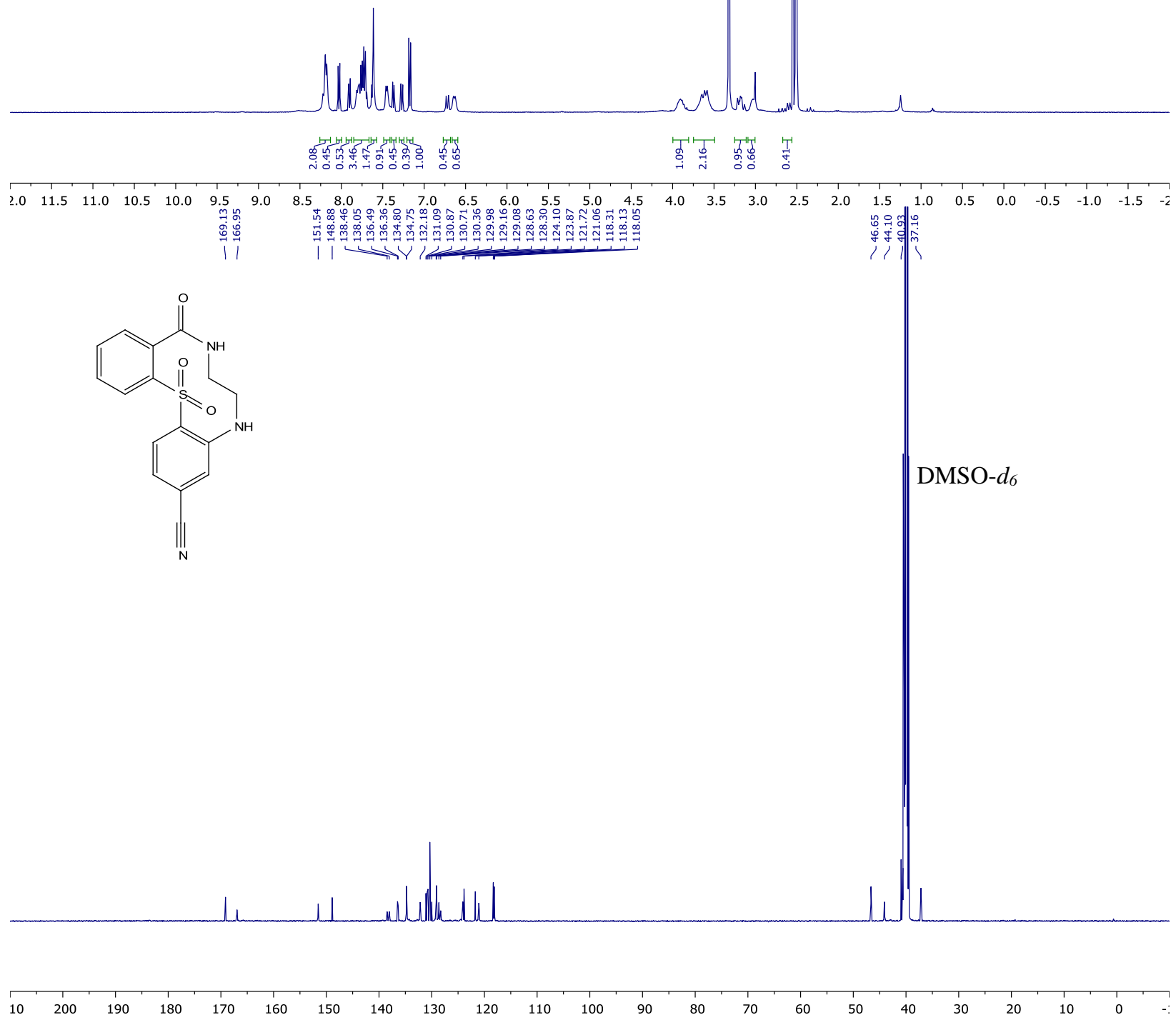

S75 
${ }^{1} \mathrm{H}\left(400 \mathrm{MHz}\right.$; in DMSO- $\left.d_{6}\right)$ and ${ }^{13} \mathrm{C}\left\{{ }^{1} \mathrm{H}\right\}\left(126 \mathrm{MHz}\right.$; in DMSO- $\left.d_{6}\right)$ NMR spectra of compound $13 \mathrm{j}$

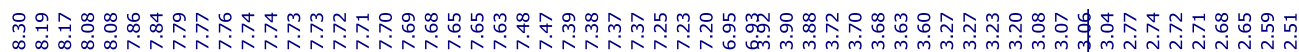<smiles>CN(C)S(=O)(=O)c1ccc(NCCNC(=O)c2ccccc2S(=O)(=O)N(C)C)cc1</smiles><smiles>C#CCC=C(C)C#C</smiles>

ind hench

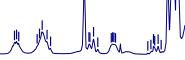

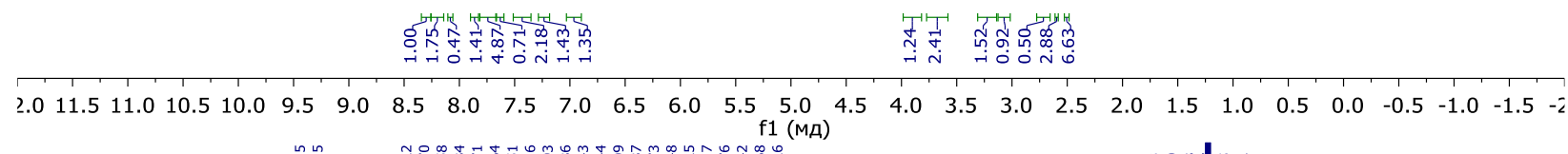

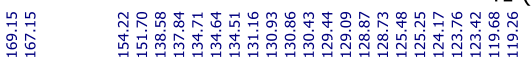

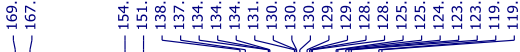<smiles>CN(C)S(=O)(=O)c1ccc(NCCNC(=O)c2ccccc2S(=O)(=O)N(C)C)cc1</smiles>

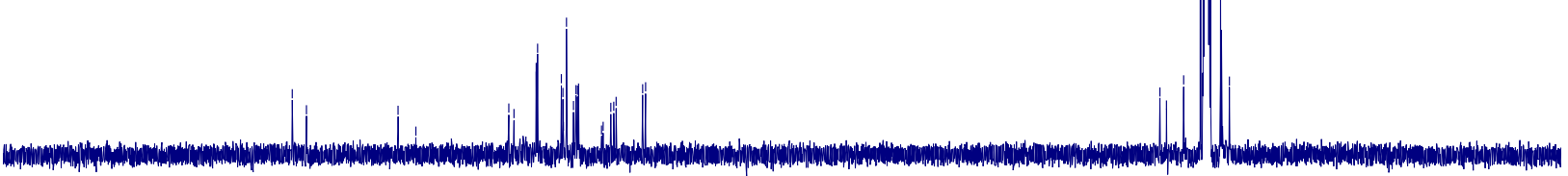

10

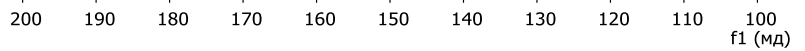

90 
${ }^{1} \mathrm{H}\left(400 \mathrm{MHz}\right.$; in DMSO- $\left.d_{6}\right)$ and ${ }^{13} \mathrm{C}\left\{{ }^{1} \mathrm{H}\right\}\left(126 \mathrm{MHz}\right.$; in DMSO- $\left.d_{6}\right)$ NMR spectra of compound $13 \mathrm{k}$

界
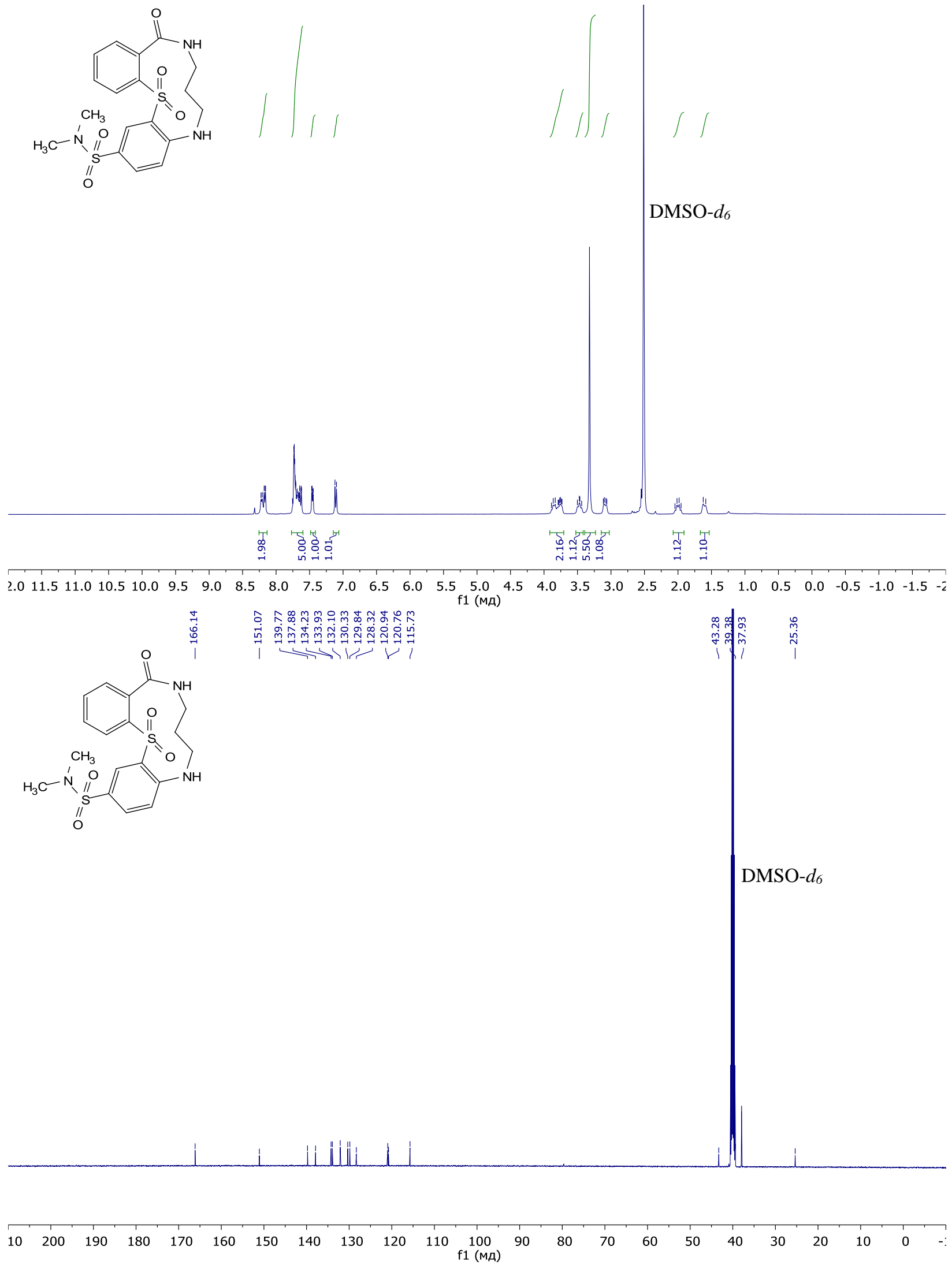

S77 
${ }^{1} \mathrm{H}\left(400 \mathrm{MHz}\right.$; in DMSO- $\left.d_{6}\right)$ and ${ }^{13} \mathrm{C}\left\{{ }^{1} \mathrm{H}\right\}\left(126 \mathrm{MHz}\right.$; in DMSO- $\left.d_{6}\right)$ NMR spectra of compound $13 \mathrm{I}$

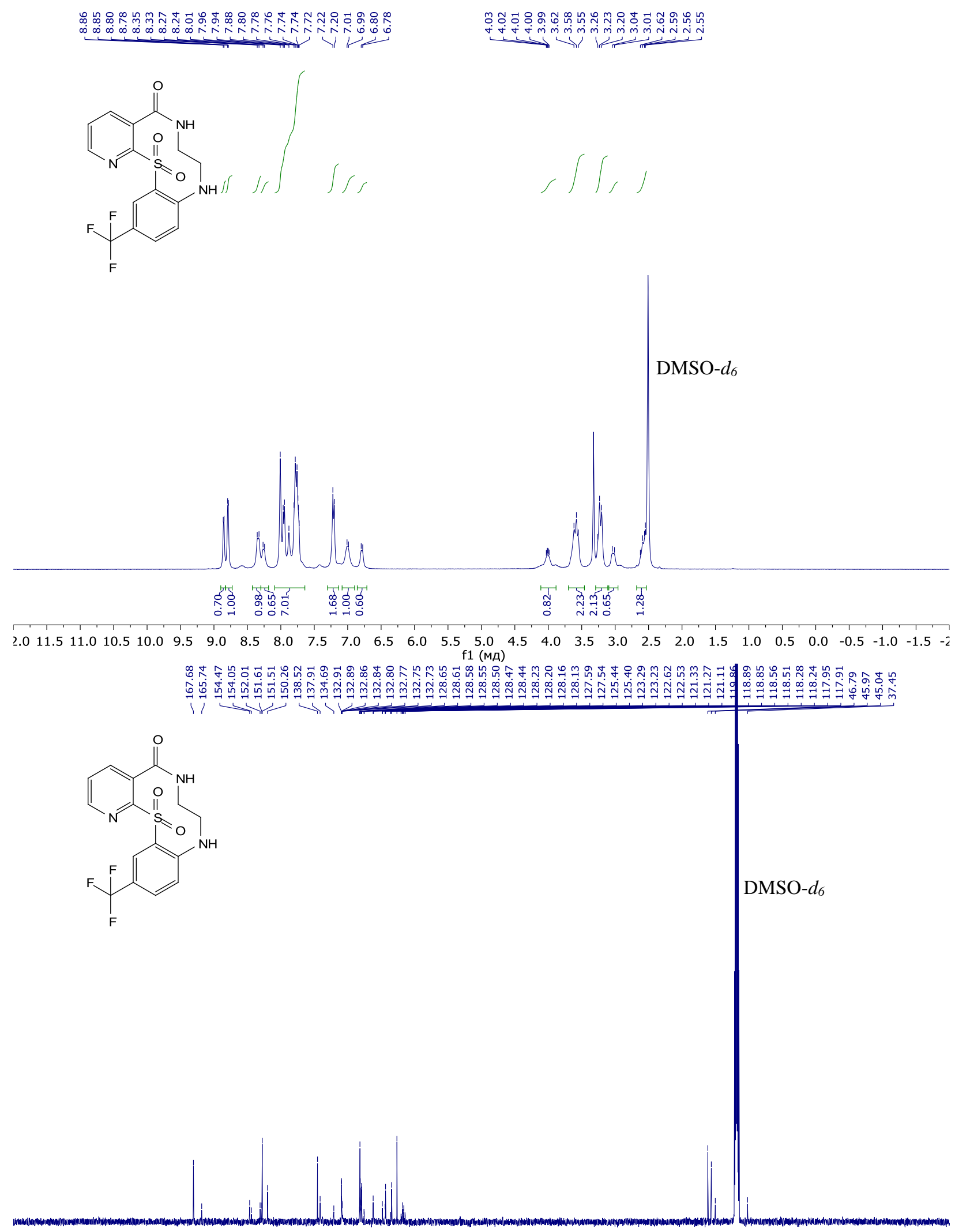

\begin{tabular}{lllllllllllllllllllllllll}
\hline 10 & 200 & 190 & 180 & 170 & 160 & 150 & 140 & 130 & 120 & 110 & 100 & 90 & 80 & 70 & 60 & 50 & 40 & 30 & 20 & 10 & 0 & -
\end{tabular} 
${ }^{1} \mathrm{H}\left(400 \mathrm{MHz}\right.$; in DMSO- $\left.d_{6}\right)$ and ${ }^{13} \mathrm{C}\left\{{ }^{1} \mathrm{H}\right\}\left(101 \mathrm{MHz}\right.$; in DMSO- $\left.d_{6}\right) \mathrm{NMR}$ spectra of compound $13 \mathrm{~m}$

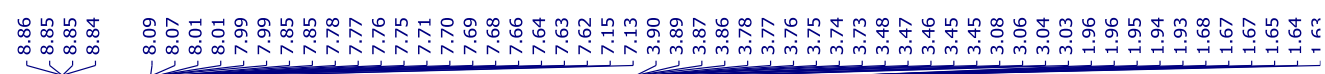<smiles>O=C(NCCNc1ccc(C(F)(F)F)cc1S(=O)(=O)O)c1ccccn1</smiles>
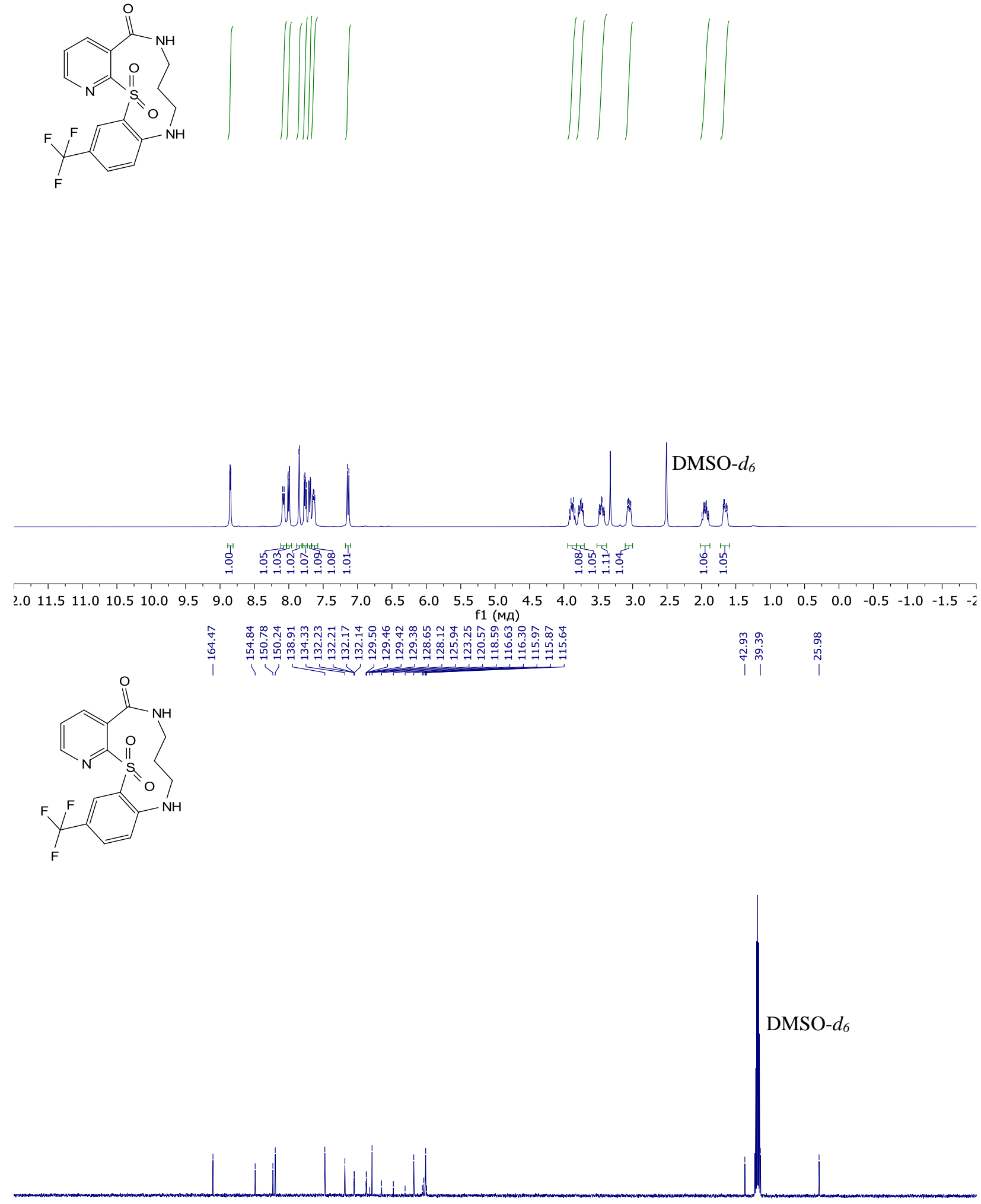

$\begin{array}{llllllllllllllllllllllllll}10 & 200 & 190 & 180 & 170 & 160 & 150 & 140 & 130 & 120 & 110 & 100 & 90 & 80 & 70 & 60 & 50 & 40 & 30 & 20 & 10 & 0 & -\end{array}$ 
${ }^{1} \mathrm{H}$ (400 MHz; in DMSO- $\left.d_{6}\right)$ and ${ }^{13} \mathrm{C}\left\{{ }^{1} \mathrm{H}\right\}\left(101 \mathrm{MHz}\right.$; in DMSO- $\left.d_{6}\right)$ NMR spectra of compound 13n

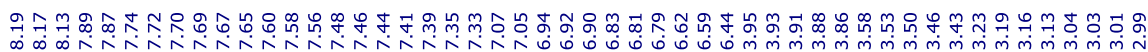<smiles></smiles>
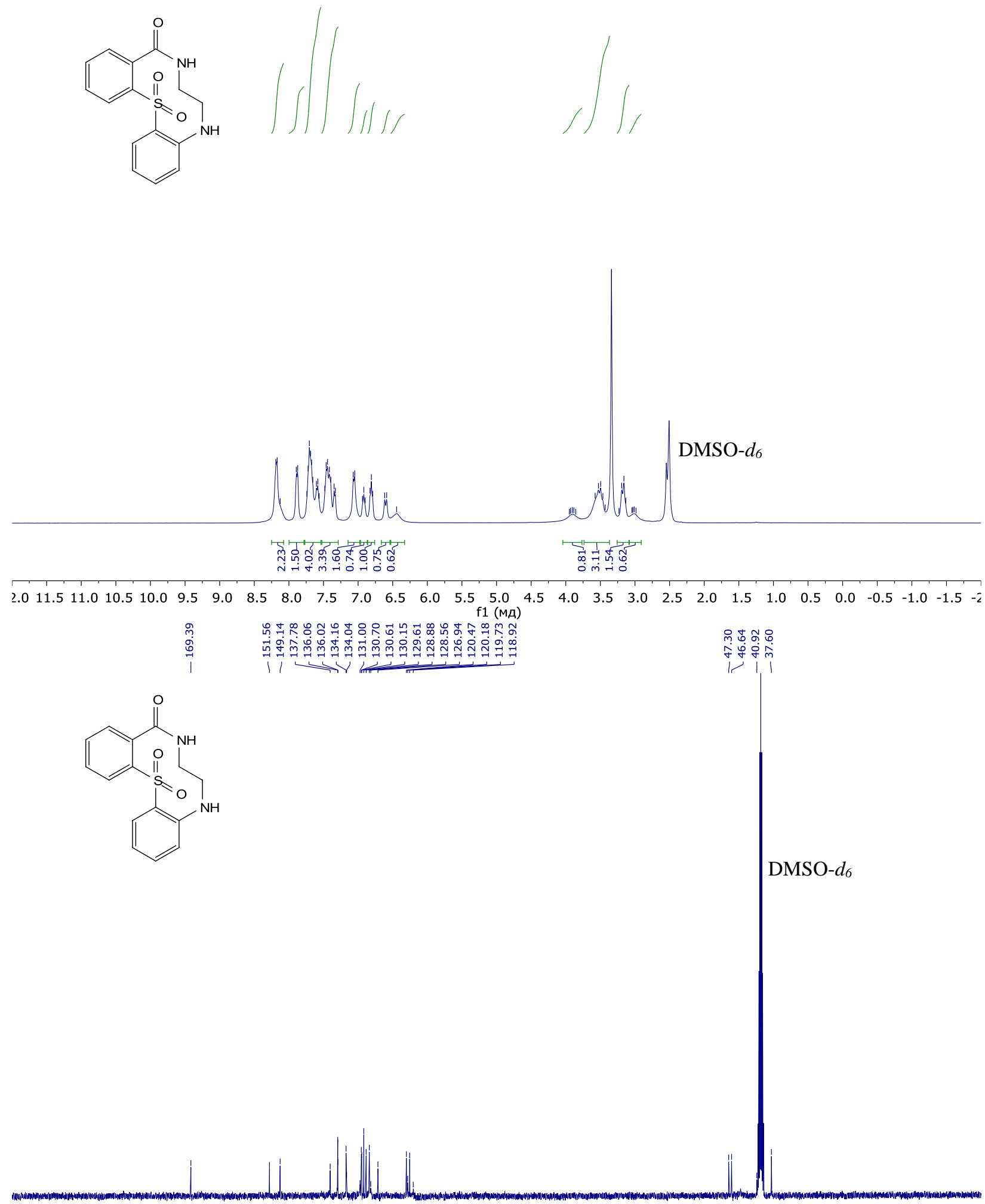

$\begin{array}{lllllllllll}10 & 200 & 190 & 180 & 170 & 160 & 150 & 140 & 130 & 120 & 110 \begin{array}{c}100 \\ \mathrm{f} 1(\mathrm{Mg})\end{array}\end{array}$ 
${ }^{1} \mathrm{H}\left(400 \mathrm{MHz}\right.$; in DMSO- $\left.d_{6}\right)$ and ${ }^{13} \mathrm{C}\left\{{ }^{1} \mathrm{H}\right\}\left(126 \mathrm{MHz}\right.$; in DMSO- $\left.d_{6}\right)$ NMR spectra of compound 14a

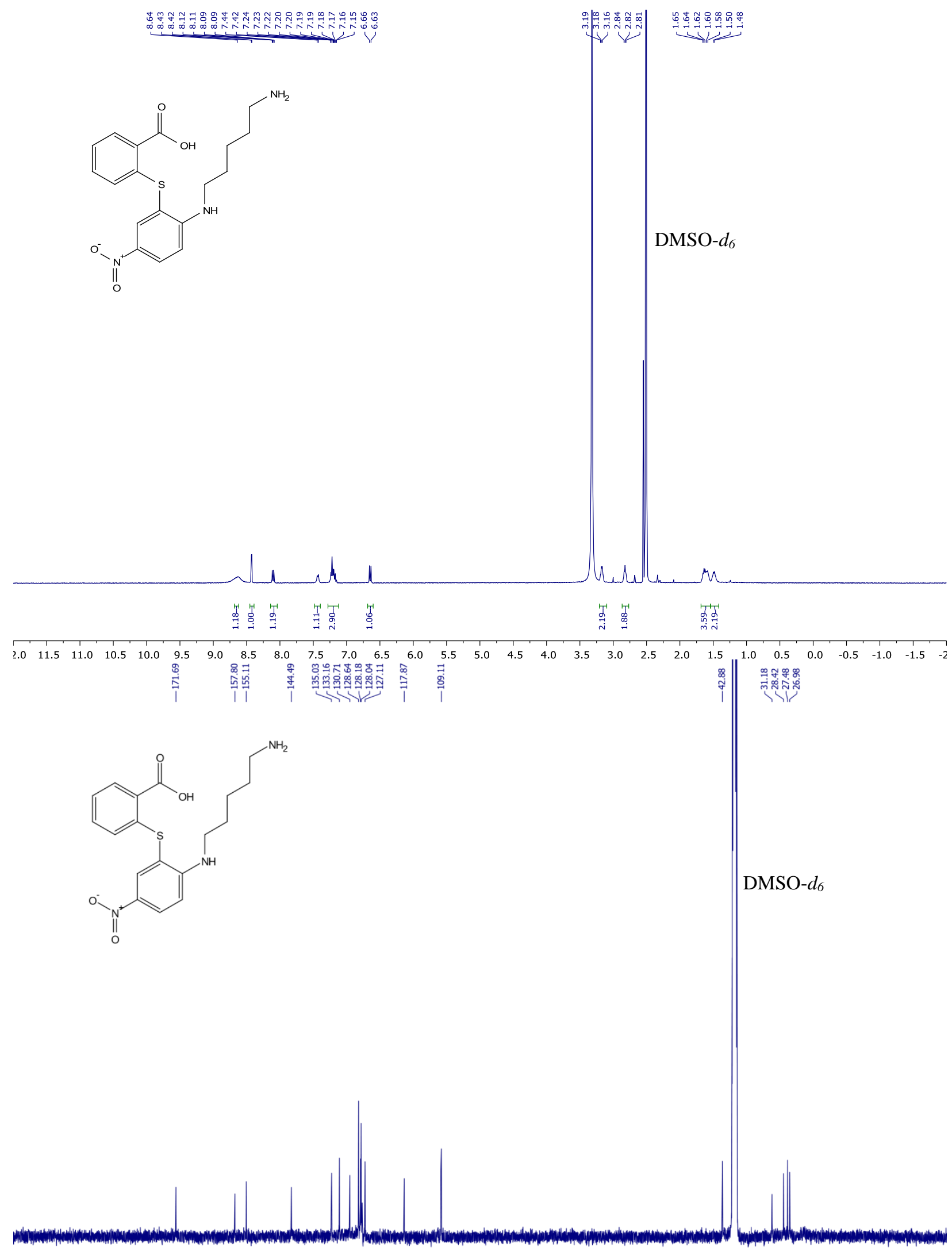

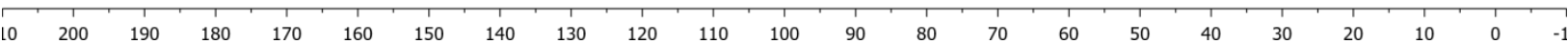


${ }^{1} \mathrm{H}\left(400 \mathrm{MHz}\right.$; in DMSO- $\left.d_{6}\right)$ and ${ }^{13} \mathrm{C}\left\{{ }^{1} \mathrm{H}\right\}\left(126 \mathrm{MHz}\right.$; in DMSO- $\left.d_{6}\right)$ NMR spectra of compound 14b

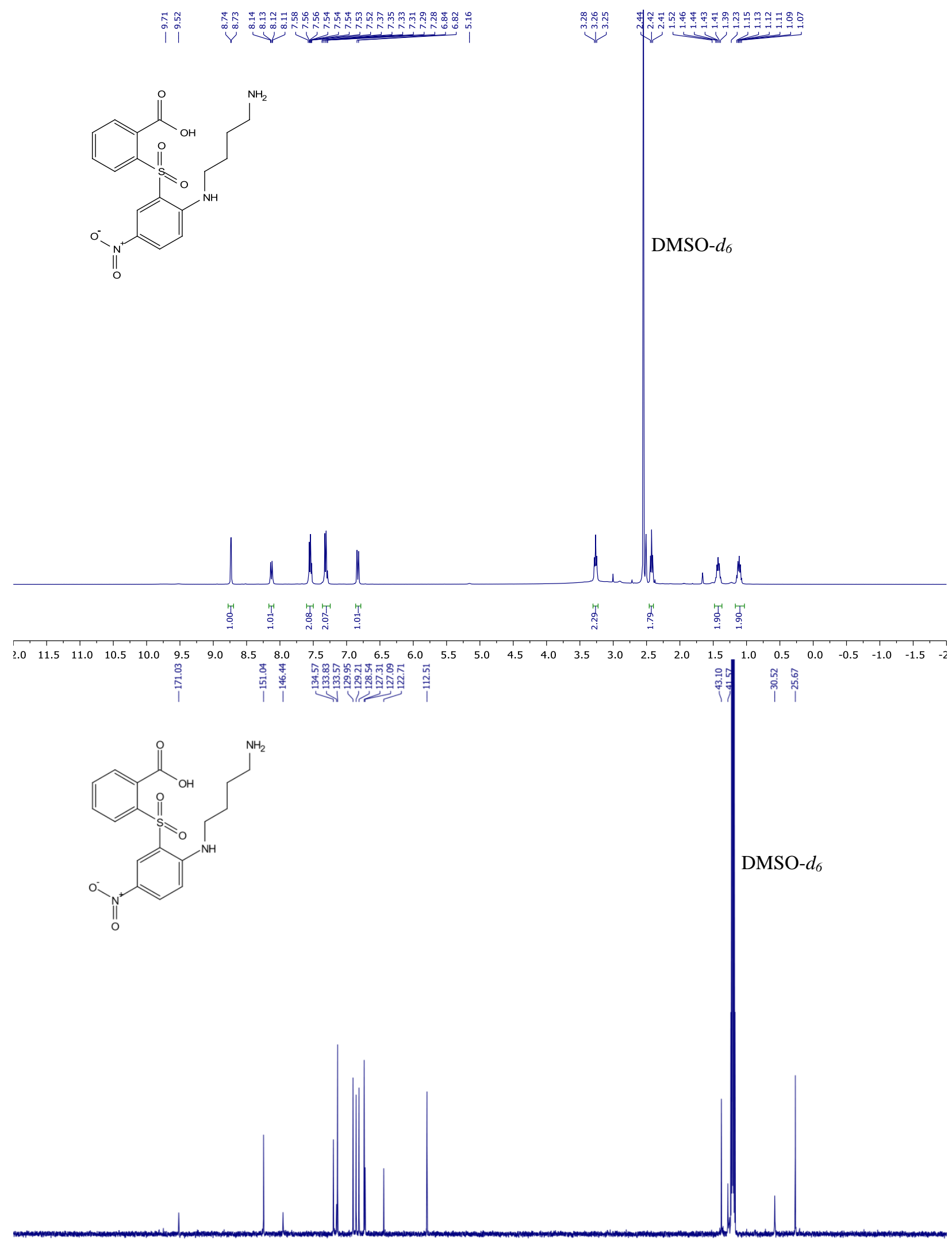

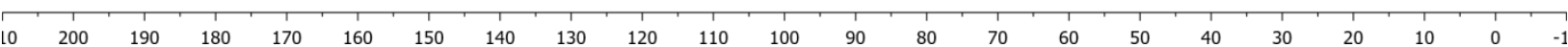


${ }^{1} \mathrm{H}\left(400 \mathrm{MHz}\right.$; in DMSO- $\left.d_{6}\right)$ and ${ }^{13} \mathrm{C}\left\{{ }^{1} \mathrm{H}\right\}\left(126 \mathrm{MHz}\right.$; in DMSO- $\left.d_{6}\right)$ NMR spectra of compound $14 \mathrm{c}$

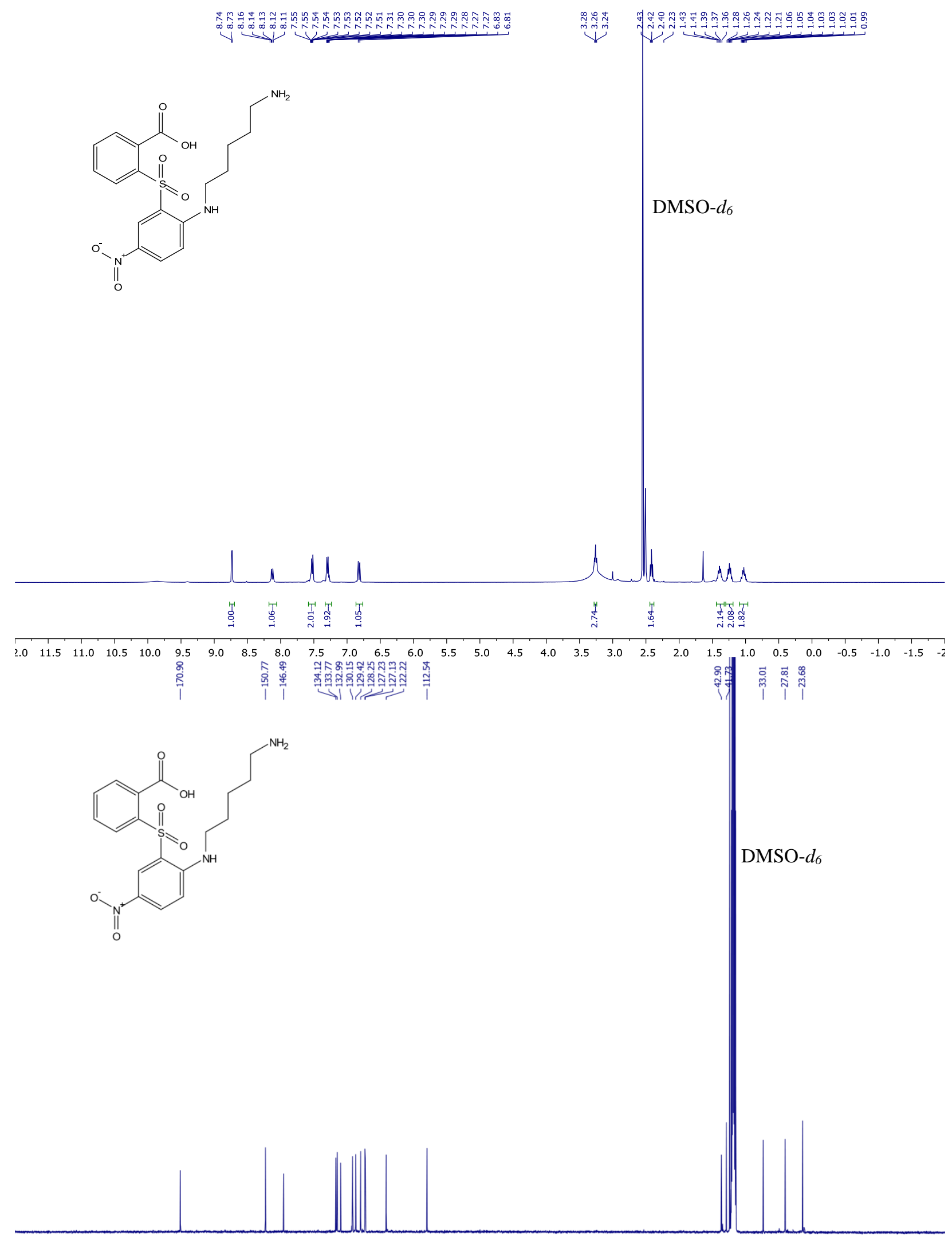

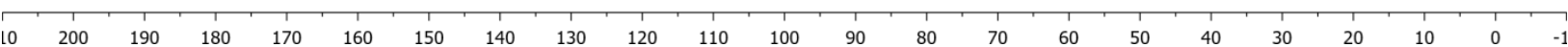




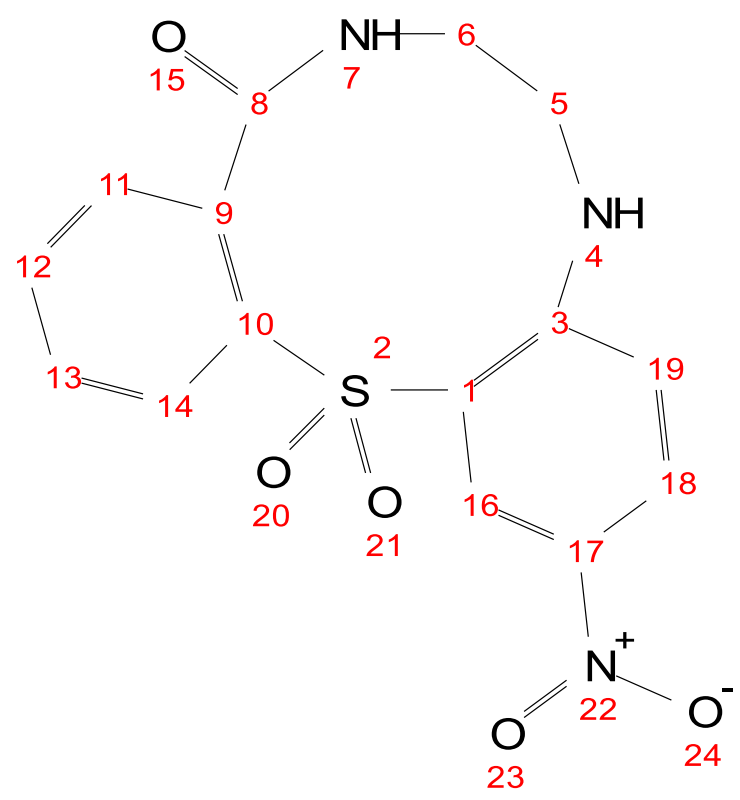

Figure S16. Structure of 13a showing the atom numbering.

Compound 13a was dissolved in DMSO- $\mathrm{d}_{6}(10 \mathrm{mM}, 0.6 \mathrm{~mL})$ and was analyzed on a $500 \mathrm{MHz}$ Bruker Avance Neo NMR instrument equipped with a TCI cryogenic probe. Two sets of ${ }^{1} \mathrm{H},{ }^{13} \mathrm{C}$ and ${ }^{15} \mathrm{~N}$ NMR signals were observed with a 2:3 ratio for the cis and trans isomer respectively. Conformational exchange of the two sets of signals was confirmed by EXSY/NOESY. For individual assignment ${ }^{1} \mathrm{H}$ and ${ }^{13} \mathrm{C}$ NMR, COSY, TOCSY, HSQC, HMBC and ${ }^{15} \mathrm{~N}$ HSQC were run at $25^{\circ} \mathrm{C}$. Variable temperature experiments were run on a $400 \mathrm{MHz}$ Agilent MR400-DD2 spectrometer equipped with an OneNMR probe for temperatures ranging from $25^{\circ} \mathrm{C}$ to $100{ }^{\circ} \mathrm{C}$.

Table S6. Assignment of the two isomers of 13a in DMSO- $\mathrm{d}_{6}$ at $25^{\circ} \mathrm{C}$.

\begin{tabular}{|c|c|c|c|c|c|}
\hline \multirow[t]{2}{*}{ Assignment } & \multirow[t]{2}{*}{ Type } & \multicolumn{2}{|c|}{ trans Isomer } & \multicolumn{2}{|c|}{ cis Isomer } \\
\hline & & $\delta-{ }^{1} \mathbf{H}[\mathrm{ppm}]$ & $\delta{ }^{-13} \mathrm{C}$ [ppm] & $\delta{ }^{-1} \mathbf{H}[\mathrm{ppm}]$ & $\delta-{ }^{13} \mathrm{C}[\mathrm{ppm}]$ \\
\hline 4 & $\mathrm{NH}$ & 7.22 & - & 7.25 & - \\
\hline 5 & $\mathrm{CH}_{2}$ & 3.72 & 43.1 & $3.25 ; 3.69$ & 45.4 \\
\hline 6 & $\mathrm{CH}_{2}$ & $3.89 ; 3.06$ & 36.6 & $2.72 ; 3.28$ & 46.4 \\
\hline 7 & $\mathrm{NH}$ & 8.30 & - & 8.24 & - \\
\hline 8 & $\mathrm{C}=\mathrm{O}$ & - & 166.7 & - & 168.8 \\
\hline 11 & $\mathrm{sp}^{2}-\mathrm{CH}$ & 7.46 & 128.8 & 7.37 & 128.9 \\
\hline 12 & $\mathrm{sp}^{2}-\mathrm{CH}$ & 7.78 & 134.8 & 7.73 & 134.8 \\
\hline 13 & $\mathrm{sp}^{2}-\mathrm{CH}$ & 7.74 & 130.5 & 7.60 & 130.8 \\
\hline 14 & $\mathrm{sp}^{2}-\mathrm{CH}$ & 8.24 & 129.1 & 7.96 & 131.6 \\
\hline 16 & $\mathrm{sp}^{2}-\mathrm{CH}$ & 8.42 & 127.8 & 8.61 & 126.1 \\
\hline 18 & $\mathrm{sp}^{2}-\mathrm{CH}$ & 8.21 & 130.7 & 8.22 & 130.7 \\
\hline 19 & $\mathrm{sp}^{2}-\mathrm{CH}$ & 7.24 & 119.2 & 7.16 & 118.2 \\
\hline
\end{tabular}




\section{EXSY/NOESY}

A NOESY spectrum for 13a in DMSO-d6 $(10 \mathrm{mM})$ was acquired with a $\mathrm{d}_{1}=2.0$ s relaxation delay and $500 \mathrm{~ms}$ mixing time, with 16 scans, $256 \times 1024$ complex points (F1 x F2) and a spectral width of $2802 \mathrm{~Hz}$. We observed chemical exchange peaks (same phase as diagonal peaks) between the corresponding $\mathrm{C}-\mathrm{H}$ protons of the two isomers, confirming slow exchange between the isomers giving rise to the two sets of signals (Figures S17 and S18).

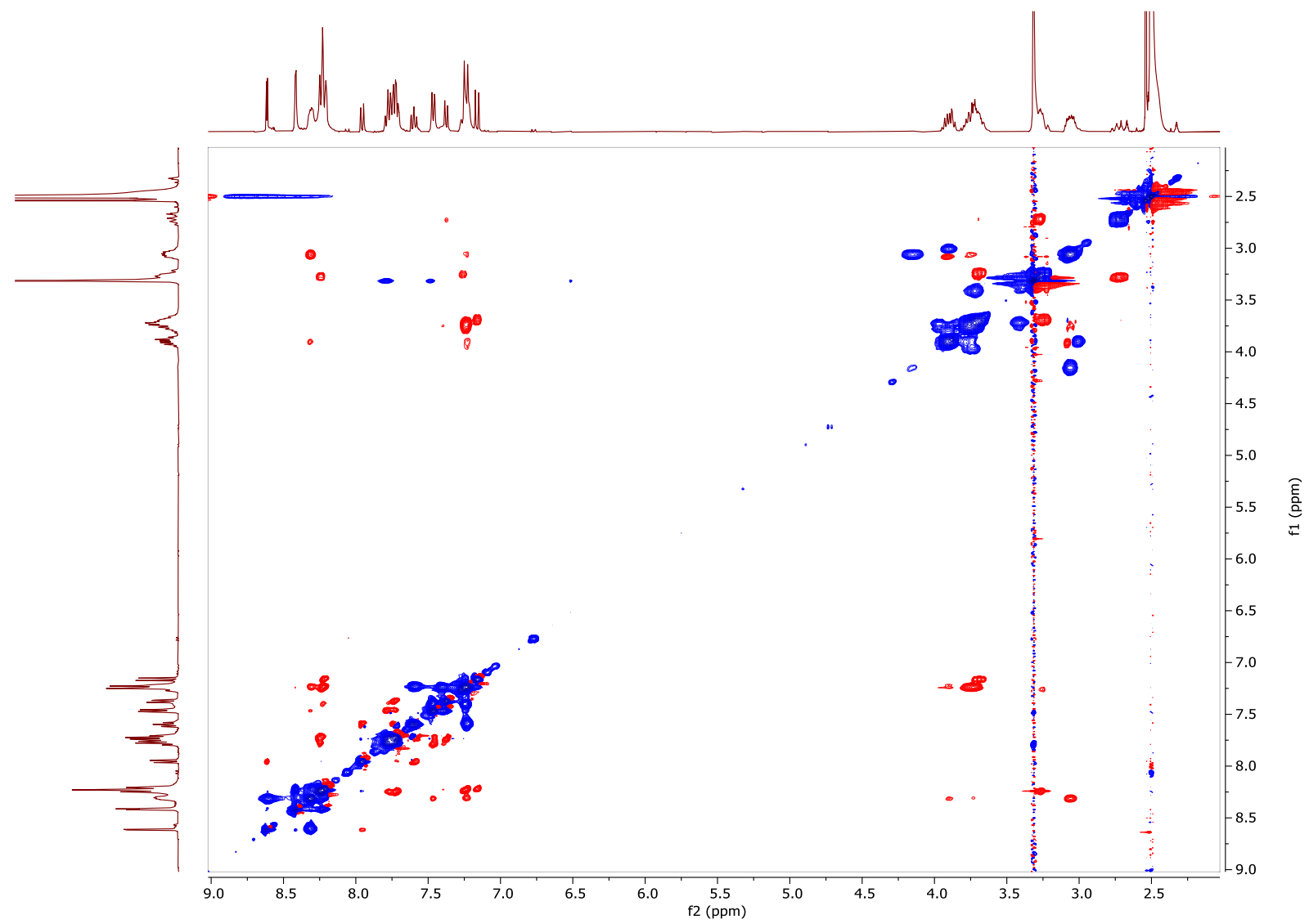

Figure S17. EXSY/NOESY spectrum of 13a in DMSO- $\mathrm{d}_{6}$. Exchange peaks between the major and minor conformer are visible as peaks in the same phase as the diagonal peaks whereas NOE cross peaks are opposite phase. 


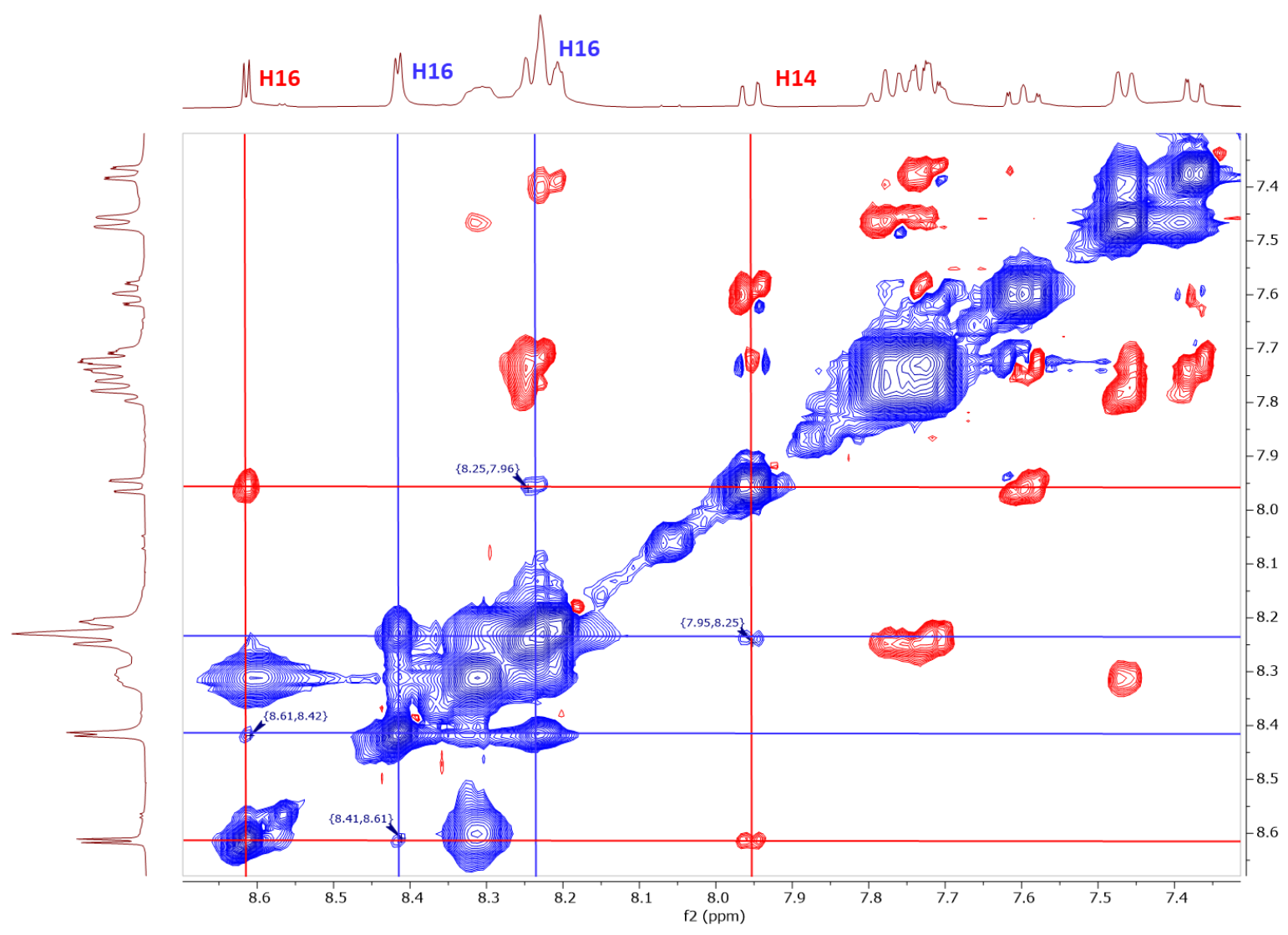

Figure S18. Extension of the EXSY/NOESY spectrum of 13a showing the same phase exchange peaks for protons H16 and H14. Blue lines indicate signals corresponding to the major trans isomer and red lines to the minor cis isomer.

\section{Variable temperature NMR}

Amide/amine temperature coefficients were determined to evaluate whether $\mathrm{NH}$ protons are involved in intramolecular hydrogen bonding. Amide or polar proton temperature coefficients of $\Delta \delta / \Delta \mathrm{T} \geq 4 \mathrm{ppb} / \mathrm{K}$ indicate solvent exposed protons in peptide/protein $\mathrm{NMR}^{1-2}$ whereas strong intramolecular hydrogen bonded protons are expected to have $\Delta \delta / \Delta \mathrm{T}<2 \mathrm{ppb} / \mathrm{K}$. This behavior is well documented for DMSO solutions. ${ }^{3}$ Chemical shift change was followed over a temperature range of $75^{\circ} \mathrm{C}\left(25-100^{\circ} \mathrm{C}\right)$ in increments of $5^{\circ} \mathrm{C}$. Due to signal overlap for $\mathrm{NH}-4$ of the cis isomer over the entire temperature range two TOCSY spectra recorded at $25^{\circ} \mathrm{C}$ and $55^{\circ} \mathrm{C}$ were used to determine its temperature coefficient. Linear regression fit for the other peaks is given as $\mathrm{R}^{2}$. Temperature coefficients deduced for all NH signals are given in table 2. Low shift changes for NH-4 in both isomers indicate that the aniline group is solvent shielded by being involved in an intramolecular hydrogen bond. For detailed discussion see main text. 
Table S7. Temperature coefficients of polar NH protons. ${ }^{a}$ Chemical shifts derived from TOCSY, recorded at $25^{\circ} \mathrm{C}$ and $55^{\circ} \mathrm{C}$. Signal overlaps prevented detection of some chemical shifts at several temperatures.

\begin{tabular}{|c|c|c|c|c|c|}
\hline \multirow[t]{2}{*}{$\mathbf{T}\left({ }^{\circ} \mathbf{C}\right)$} & \multirow[t]{2}{*}{$\mathbf{T} \mathbf{(}^{\circ}$} & \multicolumn{2}{|c|}{ trans Isomer } & \multicolumn{2}{|c|}{ cis Isomer } \\
\hline & & NH-4 & NH-7 & NH-4 & NH-7 \\
\hline 25 & 298 & 7.221 & 8.294 & 7.258 & \\
\hline 30 & 303 & & 8.274 & & \\
\hline 35 & 308 & & 8.254 & & \\
\hline 40 & 313 & & 8.235 & & 8.157 \\
\hline 45 & 318 & & & & 8.135 \\
\hline 50 & 323 & & & & 8.111 \\
\hline 55 & 328 & 7.242 & & 7.255 & 8.088 \\
\hline 60 & 333 & & & & 8.064 \\
\hline 65 & 338 & & & & 8.040 \\
\hline 70 & 343 & 7.270 & & & 8.021 \\
\hline 75 & 348 & 7.274 & 8.153 & & 7.992 \\
\hline 80 & 353 & 7.278 & 8.138 & & 7.972 \\
\hline 85 & 358 & 7.282 & 8.121 & & 7.944 \\
\hline 90 & 363 & 7.284 & 8.099 & & 7.925 \\
\hline 95 & 368 & 7.285 & 8.084 & & \\
\hline \multirow[t]{4}{*}{100} & 373 & 7.286 & 8.062 & & \\
\hline & & 0.95 & 0.99 & $\left(1^{a}\right)$ & 0.99 \\
\hline & & & & & \\
\hline & & -0.88 & -3.09 & $-0.10^{\mathrm{a}}$ & -4.63 \\
\hline
\end{tabular}

Upon increasing the temperature, the signals of the cis isomer do not change their linewidth, but their intensity decreases successively. In contrast, upon temperature increase all signals of the trans isomer broaden until $\mathrm{ca} \sim 60^{\circ} \mathrm{C}$ and thereafter sharpen again (Figure S19). No visible peaks coalesce. The change in linewidth of the major conformer and the decrease of signal intensity of the minor conformer are in agreement with a classical slow exchange behavior, as discussed in the main text. The signal of H-16 ( 8.4 ppm) of the trans isomer experiences a sigmoid chemical shift change upon temperature increase, suggesting the presence of a two-state exchange process, with its inflection point being at $\sim 60{ }^{\circ} \mathrm{C}$. 


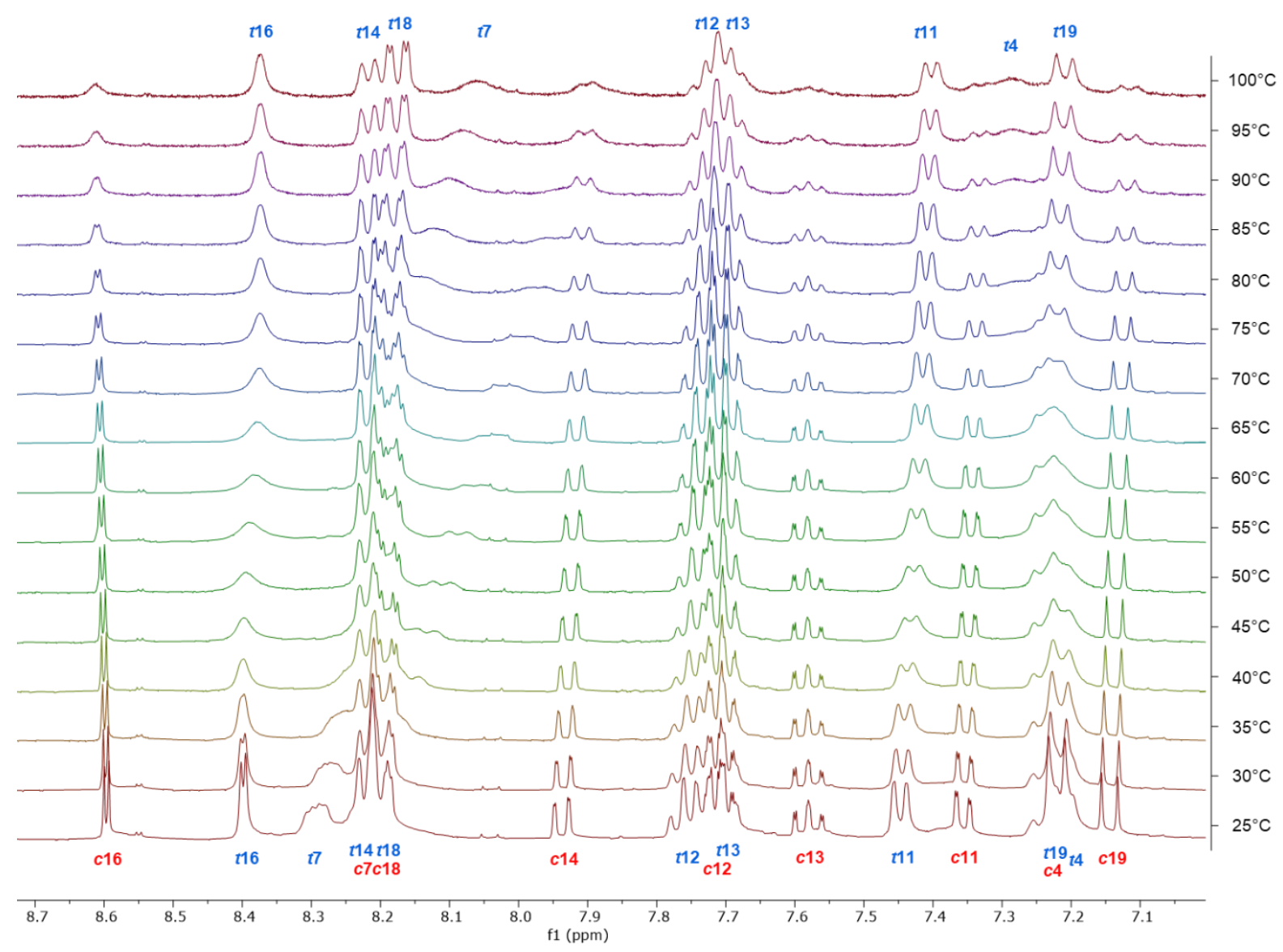

Figure S19. Variable temperature ${ }^{1} \mathrm{H}-\mathrm{NMR}$ experiment of 13a in DMSO-d 6 at $400 \mathrm{MHz}$.

\section{NOE based distance calculations}

Interproton NOE cross-peaks were observed for $\mathrm{H}-7$ to $\mathrm{H}-11$ for both isomers and for $\mathrm{H}-11$ to $\mathrm{H}-6 \mathrm{a}$ for the minor isomer only. Based on normalized integrals of NOE crosspeaks in the F2 dimension, time-averaged distances for both isomers were calculated using the Stereofitter plugin of MestreNova (v 14.1.2). NOE cross-peaks of a pair of ortho protons (cis: H11-H12; trans: H13-H14) were used as internal distance reference $(2.44 \AA)$. NOE derived distances were compared with Boltzmannweighted average distances of calculated cis and trans isomers, and aided to assign the corresponding isomer to its individual set of NMR signals (vide infra). 


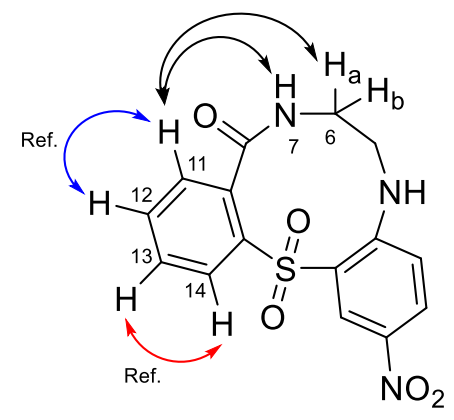

Figure S20. Structure of 13a with indicated NOE derived interproton distances. Arrows marked with 'Ref.' are used as reference distance for cis (red) and trans (blue). NOE signals of H-6a to H-11 were only visible for the cis isomer.

Table S8. NOE derived interproton distances based on normalized integrals of cross and diagonal peaks as determined by the Stereofitter plugin. ${ }^{\text {a }}$ A pair of ortho protons was used as internal reference distance for each isomer respectively

\begin{tabular}{cccccccc}
\hline Isomer & $\mathbf{H}_{\mathbf{i}}$ & $\mathbf{H}_{\mathbf{j}}$ & Diag1 & Diag2 & Cross1 & Cross2 & Dist $(\AA)$ \\
\hline cis & $\mathbf{1 3}$ & $\mathbf{1 4}$ & $\mathbf{- 6 7 6 . 6 2}$ & $\mathbf{- 9 8 8 . 0 9}$ & $\mathbf{3 4 . 8 4}$ & $\mathbf{2 8 . 3 8}$ & $\mathbf{2 . 4 4}^{\mathbf{a}}$ \\
& 7 & 11 & - & -984.52 & - & 6.29 & 3.31 \\
& $6 \mathrm{a}$ & 11 & -529.44 & -890.94 & - & 9.05 & 3.07 \\
\hline trans & $\mathbf{1 1}$ & $\mathbf{1 2}$ & $\mathbf{- 1 1 3 7 . 4 3}$ & $\mathbf{- 1 6 9 7 . 5 4}$ & $\mathbf{4 3 . 9 4}$ & $\mathbf{5 3 . 9 0}$ & $\mathbf{2 . 4 4}^{\mathbf{a}}$ \\
& 7 & 11 & -1286.37 & -1313.68 & - & 14.44 & 2.91
\end{tabular}

\section{Monte-Carlo conformational search}

Monte Carlo conformational searches for the cis and trans isomers were performed individually using 10.000 Monte-Carlo steps followed by 5.000 minimization steps. The OPLS3e force field was used with the GB/SA continuum water solvation model. All conformers within a $42 \mathrm{~kJ} / \mathrm{mol}$ energy window from the lowest energy conformer were retained. The conformational preferences of the cis and trans isomer were assessed by restraining the amide torsion angle $(\mathrm{O}=\mathrm{C}-\mathrm{N}-\mathrm{H})$ to $0^{\circ} \pm 0^{\circ}$ for the cis and to $180^{\circ} \pm 0^{\circ}$ for the trans isomer using a $1.000 \mathrm{~kJ} / \mathrm{mol}$ force constant. Redundant conformer elimination obtained an even coverage of rotatable bonds with an individual atom RMSD $\geq 2.0 \AA$. The conformational search provided 5 conformers for the cis and 4 for the trans isomer.

Calculated relative potential energies based on the OPLS3e force field were used to determine the Boltzmann distribution for resulting conformers following

$$
p_{i}=\frac{e^{-\Delta \varepsilon_{i} / k T}}{\sum_{j} e^{-\Delta \varepsilon_{j} / k T}}
$$

where $p_{i}$ is the molar fraction of conformer $\mathrm{i}, \mathrm{j}$ is the total number of conformers found and $\Delta \varepsilon_{j}$ is the energy difference for all conformers in regard to the lowest energy conformer for each isomer. ${ }^{4}$ Boltzmann populations were used to evaluate weighted arithmetic means for interproton distances $(\mathrm{H}-$ 7 to H-11 and H-6a to H-11) in the ensembles of the cis and trans isomers (Table S9) and were used to assign individual NMR signals to its corresponding isomer. The long average distance (4.81 $\AA$ ) of $\mathrm{H}$ 6a to 11 calculated for the trans isomer explains why no NOE signal could be observed for this isomer as it is close to the maximum distance detectable (5 $\AA)$ with 2D-NOESY spectroscopy. 
Table S9. Weighted averages of interproton distances for each conformer based on Boltzmann percentages of conformers at 298K. Energies given are calculated by the force field OPLS3e used for the Monte-Carlo conformational search. The color code of the conformations corresponds to the colors of the conformers in Figure S21.

\begin{tabular}{|c|c|c|c|c|c|c|c|c|}
\hline Isomer & $\begin{array}{c}\# \\
\text { Conformer }\end{array}$ & $\begin{array}{c}\text { Potential energy } \\
\text { OPLS3e } \\
{[\mathrm{kJ} / \mathrm{mol}]}\end{array}$ & $\begin{array}{c}\text { Relative energy } \\
\text { OPLS3e } \\
{[\mathrm{kJ} / \mathrm{mol}]}\end{array}$ & $\begin{array}{c}\text { Boltzmann } \\
\text { fractions } \\
p_{i}\end{array}$ & $\begin{array}{c}\text { Distance } \\
\text { H7 - H11 }\end{array}$ & $\begin{array}{l}\text { Weighted } \\
\text { distance }\end{array}$ & $\begin{array}{c}\text { Distance } \\
\text { H6a - } \\
\text { H11 }\end{array}$ & $\begin{array}{c}\text { Weighted } \\
\text { distance }\end{array}$ \\
\hline \multirow[t]{6}{*}{ cis } & 1 & 189.64 & 0 & 0.9758 & 3.82 & 3.73 & 3.20 & 3.11 \\
\hline & 2 & 198.822 & 9.182 & 0.0240 & 4.01 & 0.10 & 3.56 & 0.09 \\
\hline & 3 & 210.047 & 20.407 & 0.0003 & 3.82 & $9.9 \mathrm{E}-4$ & 2.78 & 0.000718 \\
\hline & 4 & 220.167 & 30.527 & 4.3476E-06 & 3.92 & $1.7 \mathrm{E}-05$ & 4.46 & $1.94 \mathrm{E}-05$ \\
\hline & 5 & 228.743 & 39.104 & $1.3639 \mathrm{E}-07$ & 3.92 & 5.3E-07 & 4.51 & $6.16 \mathrm{E}-07$ \\
\hline & & & & & $\begin{array}{c}\text { Weighted } \\
\text { Average }\end{array}$ & 3.82 & & 3.20 \\
\hline \multirow[t]{5}{*}{ trans } & 1 & 190.561 & 0 & 0.9839 & 3.22 & 3.17 & 4.80 & 4.72 \\
\hline & 2 & 200.817 & 10.256 & 0.01567 & 3.54 & $5.54 \mathrm{E}-2$ & 5.31 & 0.08 \\
\hline & 3 & 209.944 & 19.383 & 0.0004 & 3.98 & $1.57 \mathrm{E}-3$ & 5.07 & 0.001998 \\
\hline & 4 & 220.014 & 29.453 & $6.7629 \mathrm{E}-06$ & 3.22 & 2.2E-05 & 4.80 & $3.24 \mathrm{E}-05$ \\
\hline & & & & & $\begin{array}{c}\text { Weighted } \\
\text { Average }\end{array}$ & 3.22 & & 4.81 \\
\hline
\end{tabular}


A

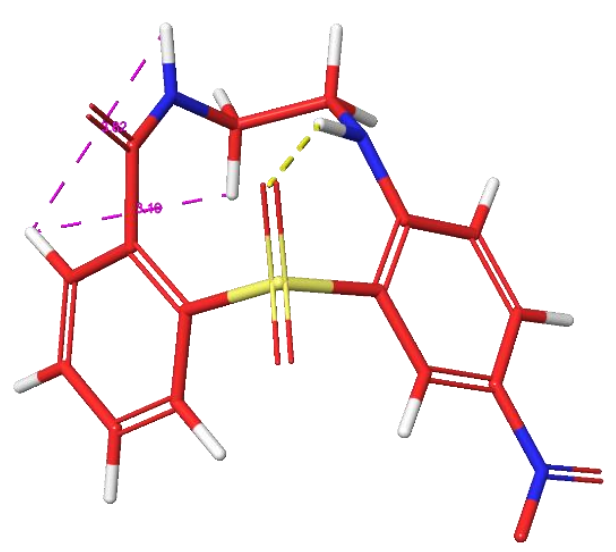

B

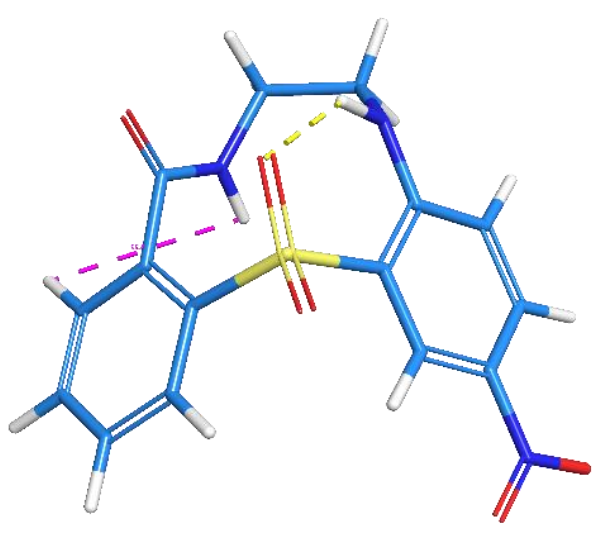

C

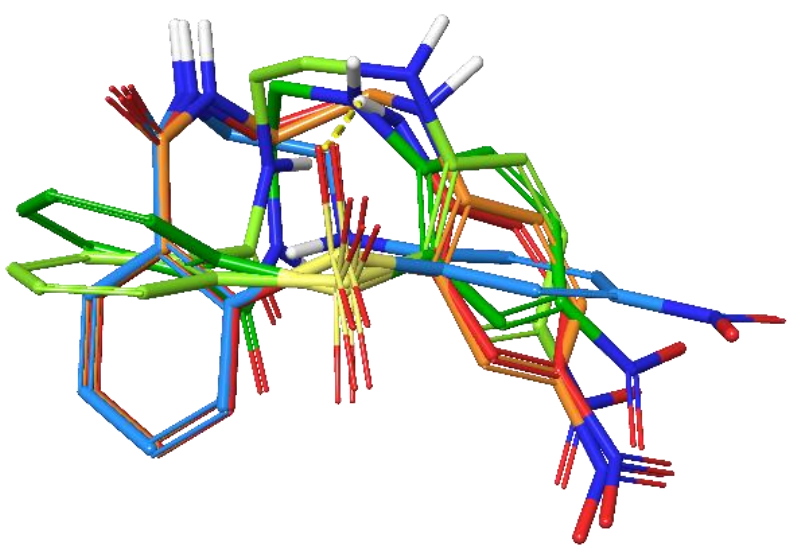

D

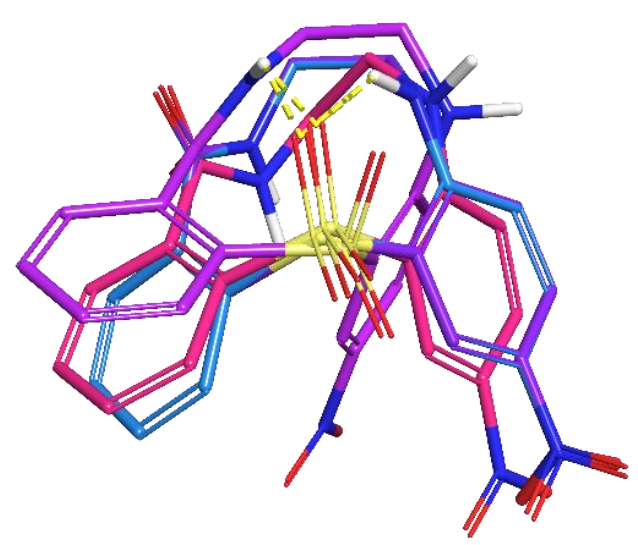

Figure S21. A/C: Lowest energy conformers calculated for the cis $(A)$ and trans $(B)$ isomer. Hydrogen bonds are indicated by yellow dotted lines. Experimentally determined interproton distances are indicated by purple dotted lines. B/D: Superimposed conformers for the cis $(B)$ and trans $(D)$ isomer. Conformational families are indicated with same color shades. 


\section{NMR spectra}

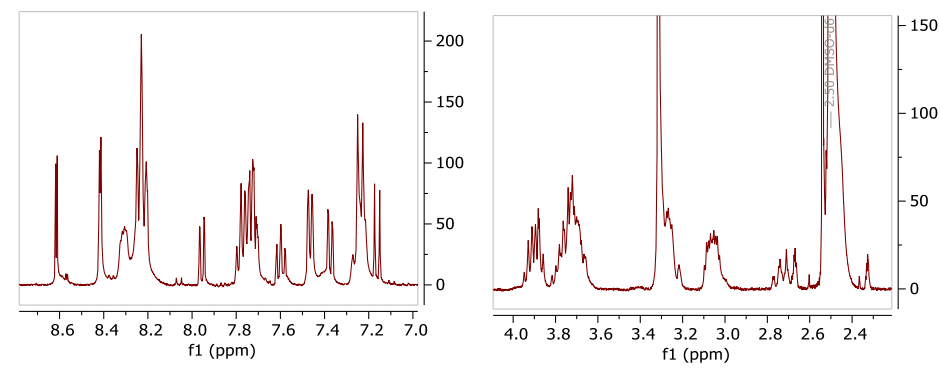

Whemath

3400

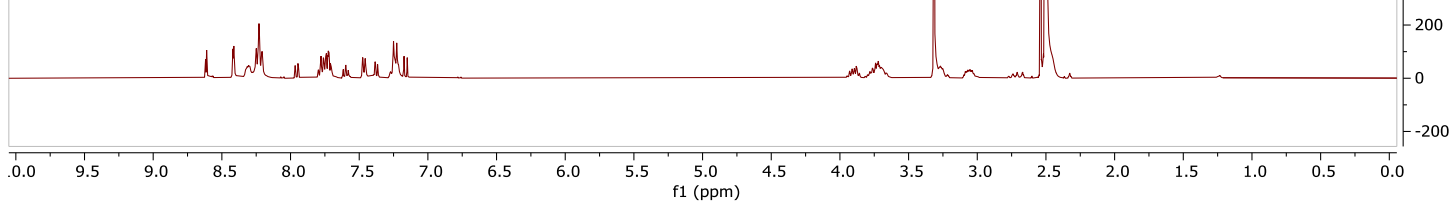

Figure S22. ${ }^{1} \mathrm{H}-\mathrm{NMR}$ of $\mathbf{1 3 a}$

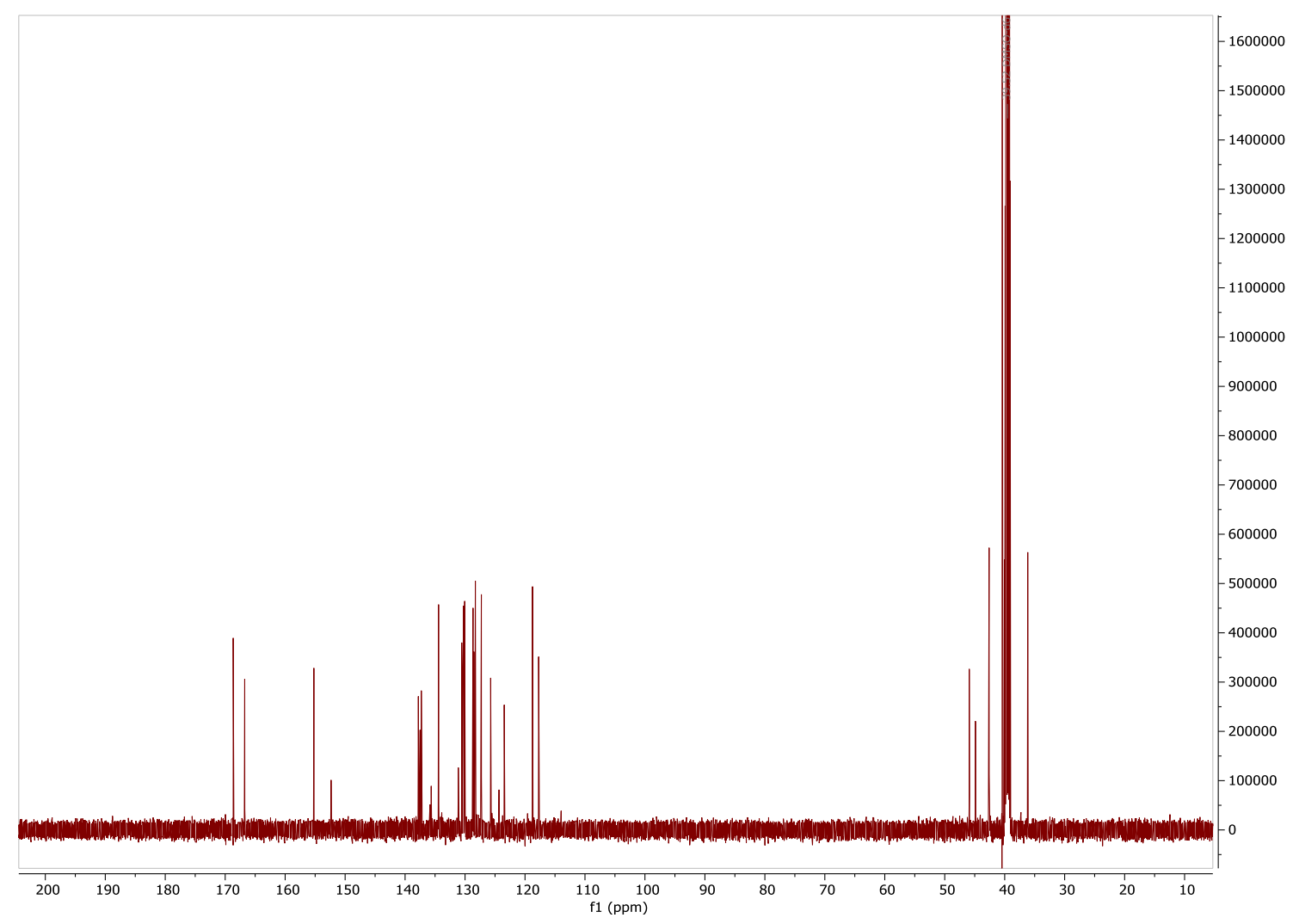

Figure S23. ${ }^{13} \mathrm{C}-\mathrm{NMR}$ of $\mathbf{1 3 a}$ 


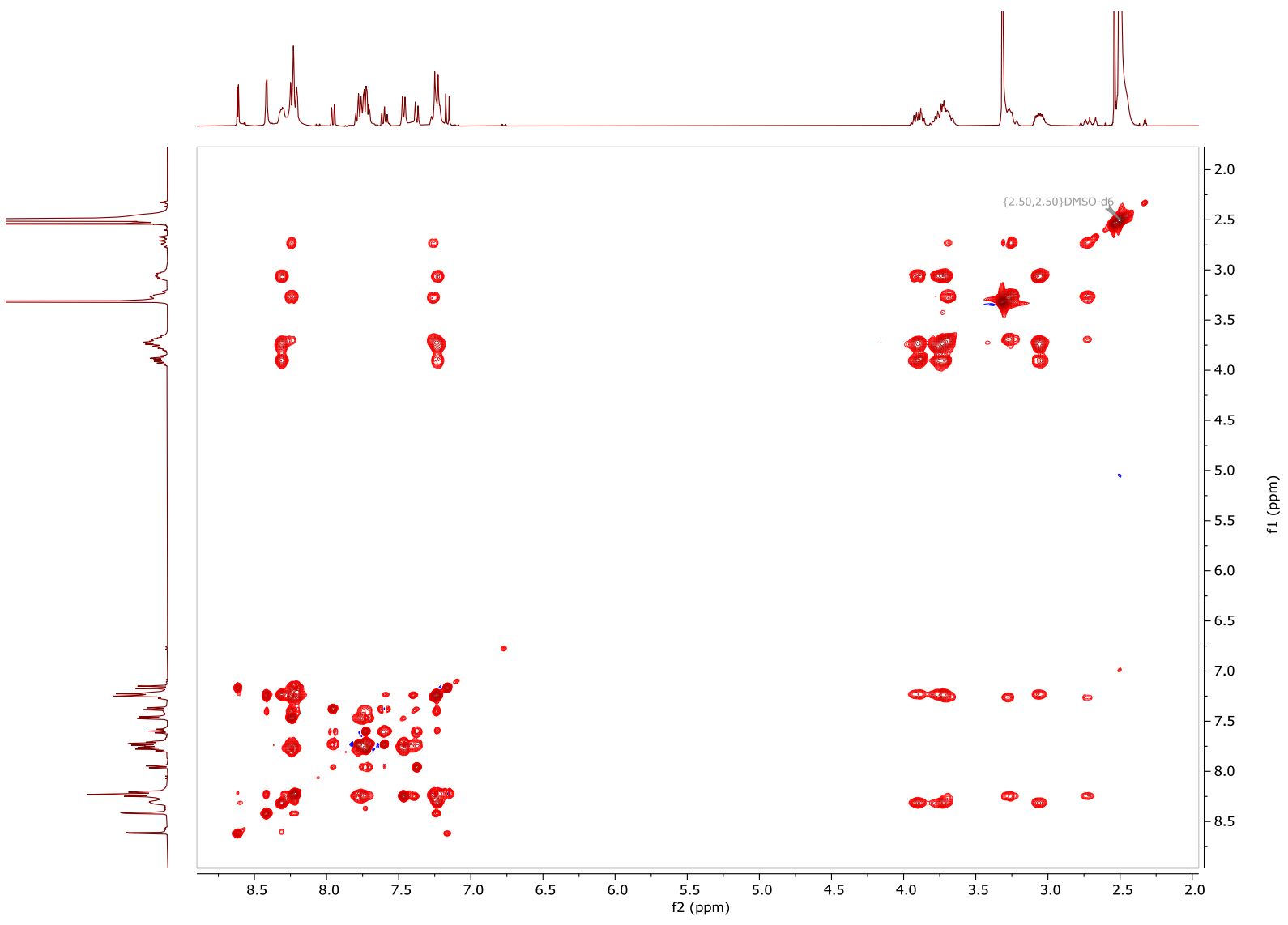

Figure S24. TOCSY spectrum of 13a

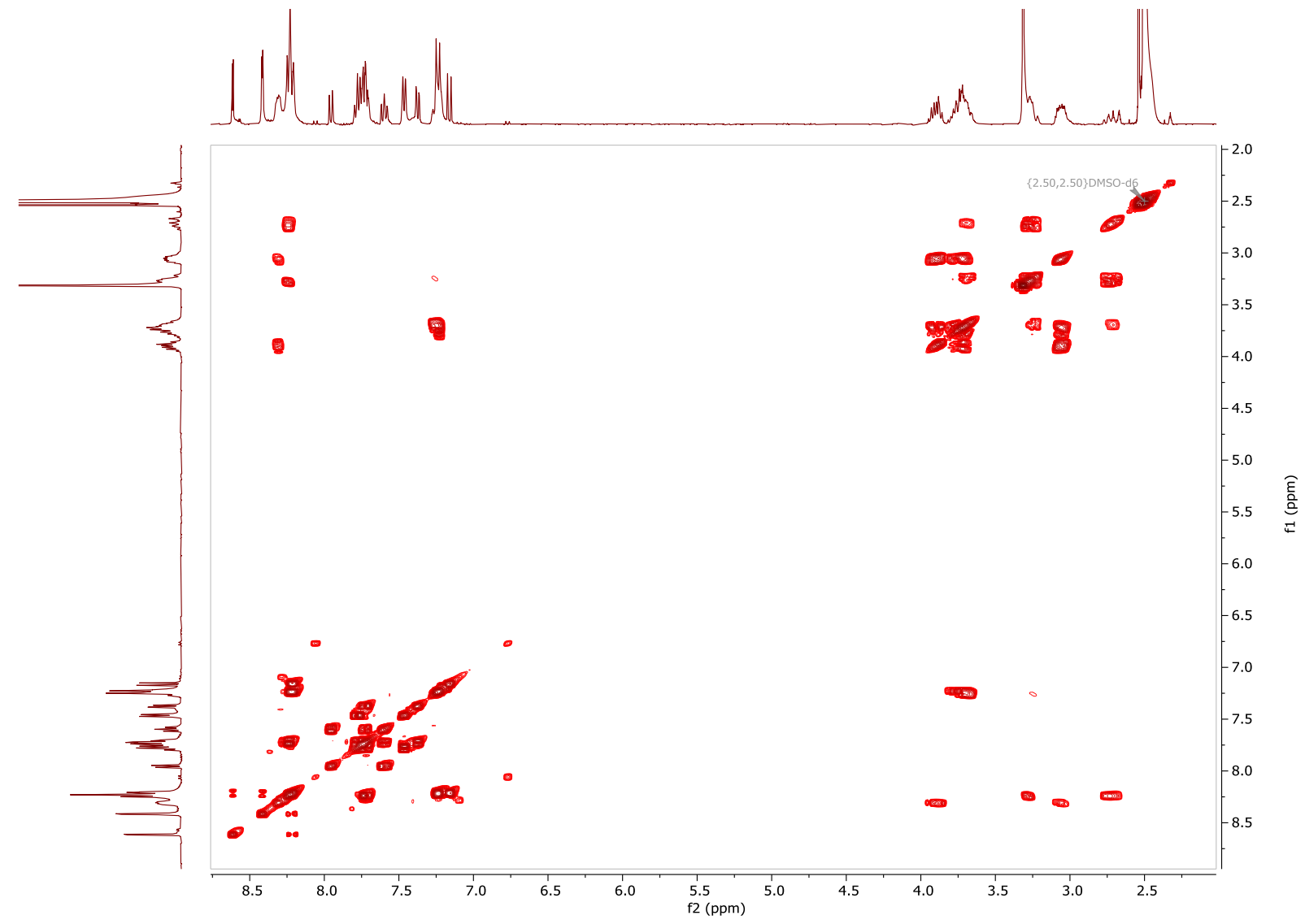

Figure S25. COSY spectrum of 13a 


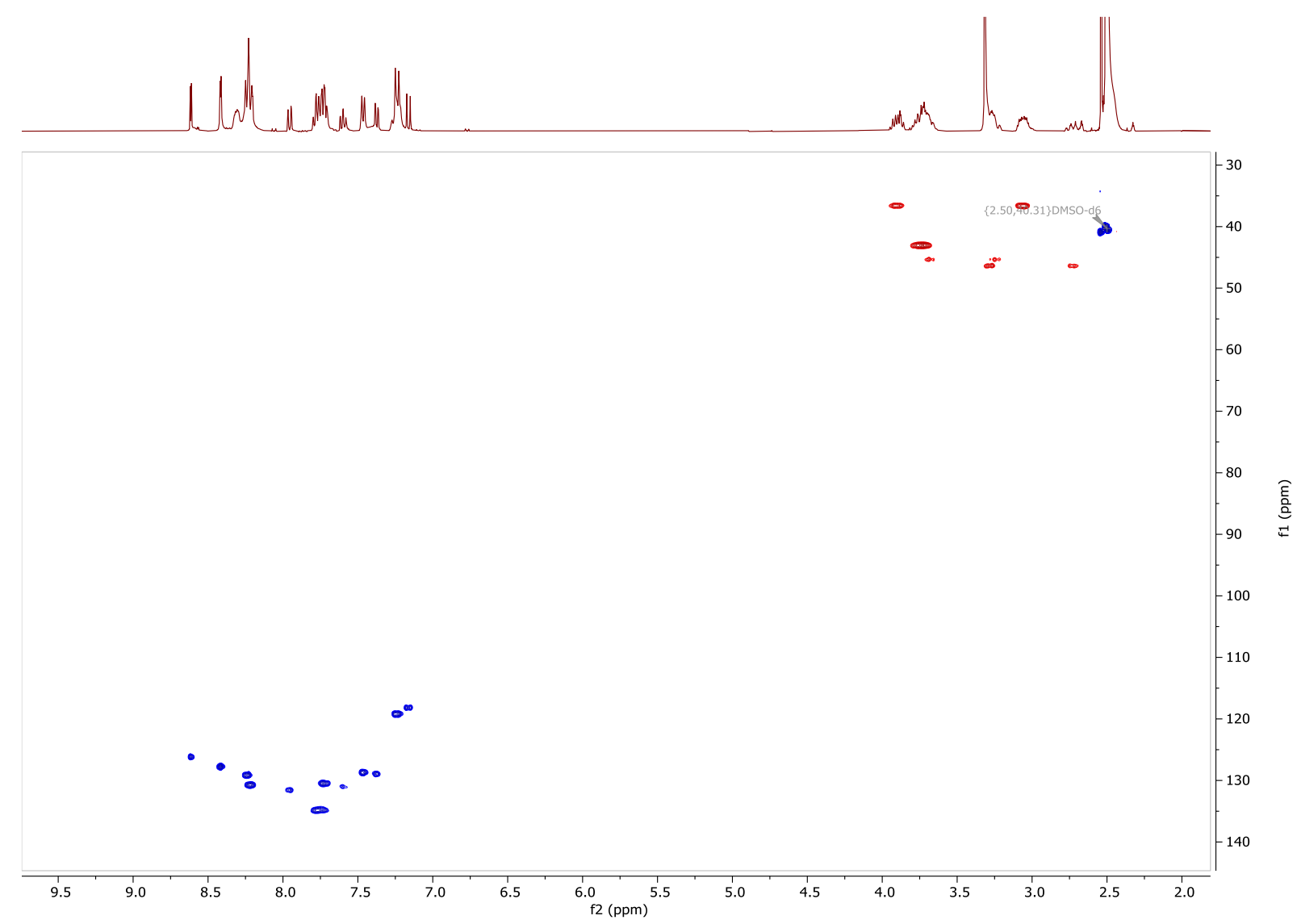

Figure S26. ${ }^{1} \mathrm{H}-{ }^{13} \mathrm{C}$ HSQC spectrum of $\mathbf{1 3 a}$

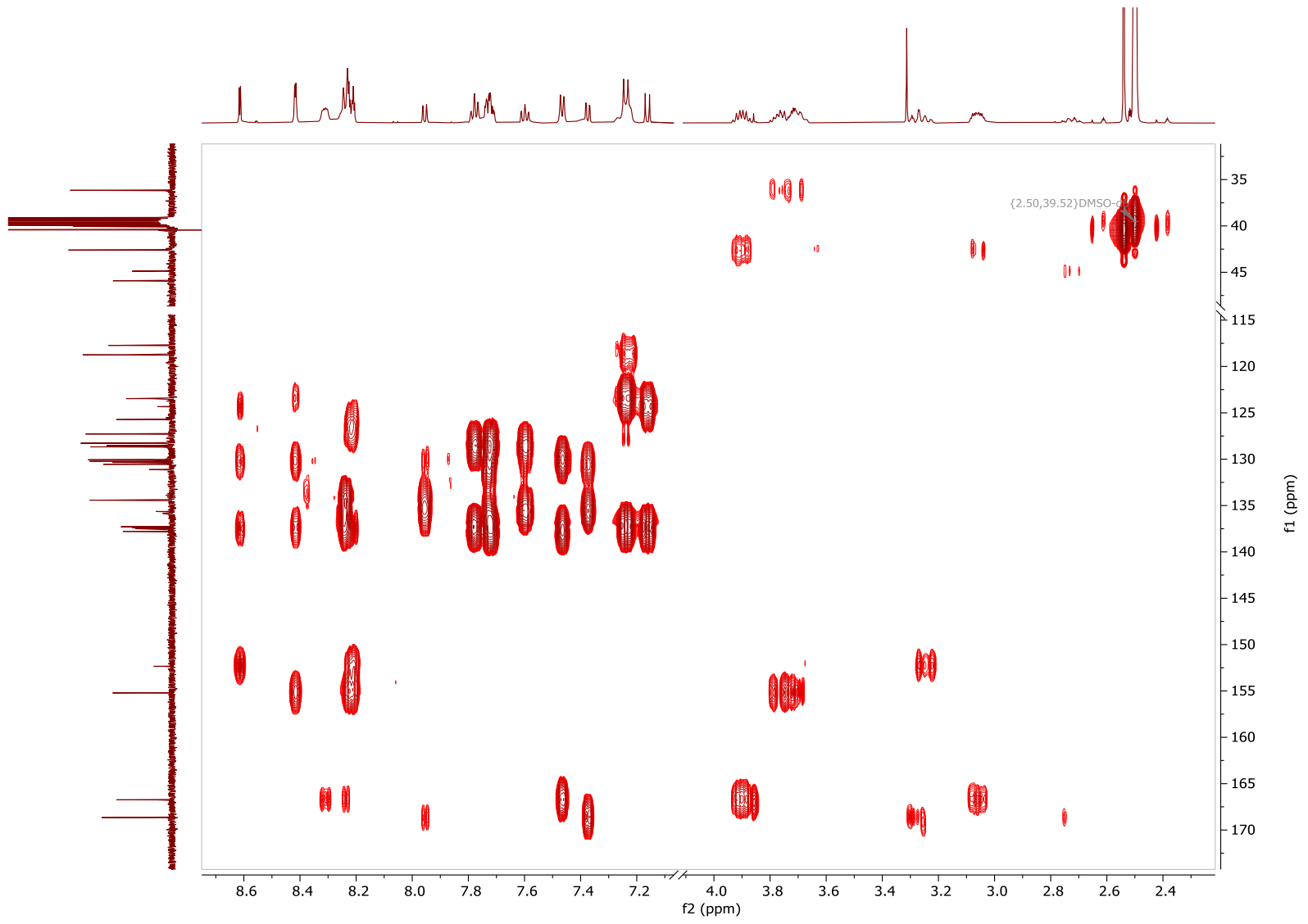

Figure S27. HMBC spectrum of 13a 


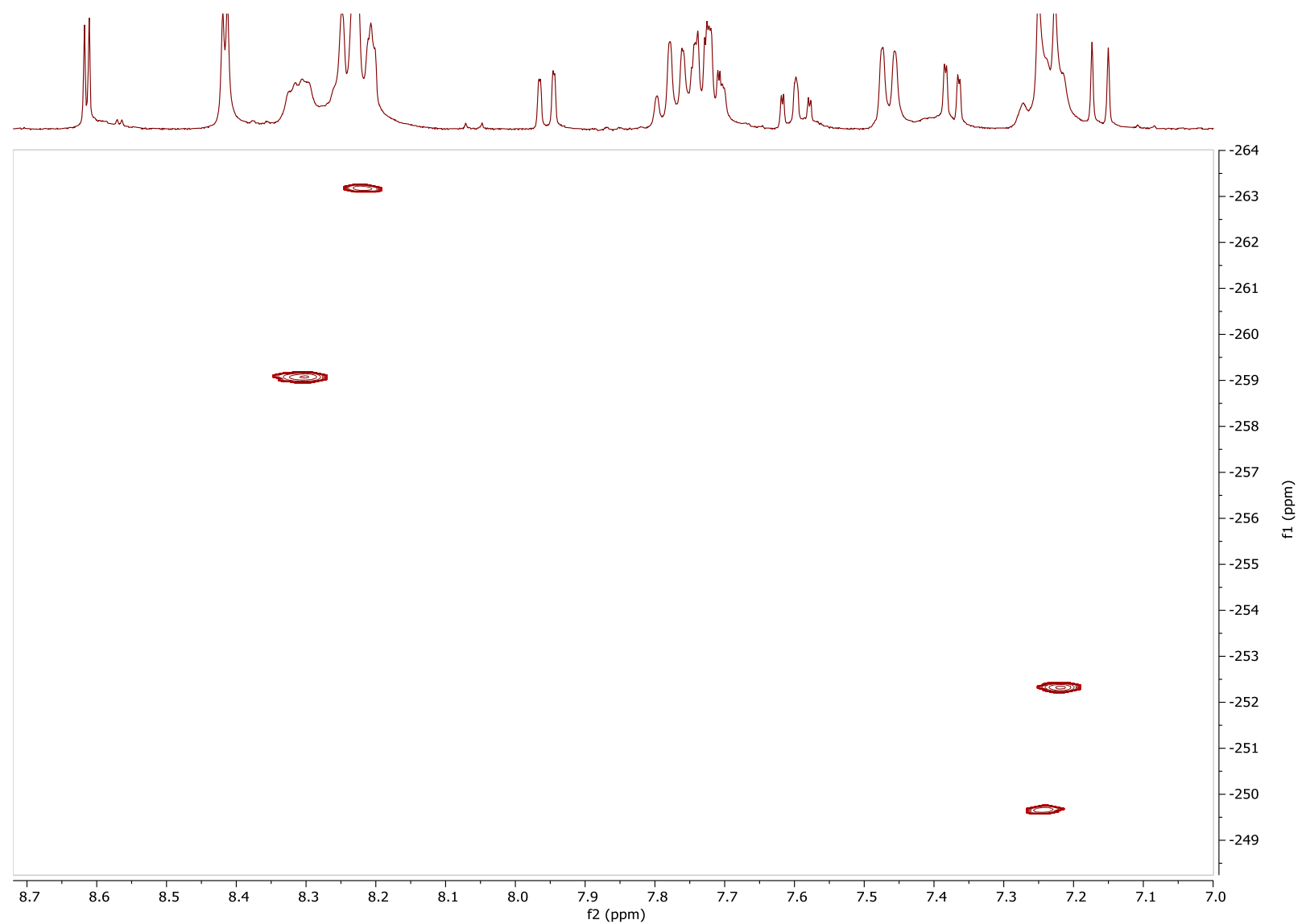

Figure S28. ${ }^{1} \mathrm{H}-{ }^{15} \mathrm{~N}$ HSQC spectrum of $\mathbf{1 3 a}$

\section{Ensemble atom coordinates}

\begin{tabular}{lccc}
\multicolumn{4}{l}{ REU_651_trans_1 } \\
O & -0.80980 & 7.65310 & 4.24970 \\
$\mathrm{C}$ & -1.35960 & 7.63920 & 5.35260 \\
$\mathrm{C}$ & -2.84700 & 7.77210 & 5.45530 \\
$\mathrm{C}$ & -3.71670 & 6.67530 & 5.24370 \\
$\mathrm{C}$ & -5.09960 & 6.88540 & 5.33550 \\
$\mathrm{C}$ & -5.61720 & 8.14750 & 5.61760 \\
$\mathrm{C}$ & -4.75770 & 9.22250 & 5.82070 \\
$\mathrm{C}$ & -3.38020 & 9.03530 & 5.74040 \\
$\mathrm{~N}$ & -0.73620 & 7.39730 & 6.51990 \\
$\mathrm{C}$ & 0.62380 & 6.86240 & 6.61850 \\
$\mathrm{C}$ & 0.66990 & 5.38200 & 7.06390 \\
$\mathrm{~N}$ & -0.33340 & 4.51610 & 6.43220 \\
$\mathrm{C}$ & -1.50240 & 4.20310 & 7.15410 \\
$\mathrm{C}$ & -2.79550 & 4.33210 & 6.60000 \\
$\mathrm{C}$ & -3.91210 & 3.99540 & 7.37540 \\
$\mathrm{C}$ & -3.78570 & 3.51460 & 8.68440 \\
$\mathrm{~N}$ & -4.98310 & 3.16550 & 9.48730 \\
$\mathrm{O}$ & -4.82320 & 2.74530 & 10.63000 \\
$\mathrm{O}$ & -6.09320 & 3.30910 & 8.98230 \\
$\mathrm{C}$ & -2.49960 & 3.37160 & 9.21440 \\
$\mathrm{C}$ & -1.37940 & 3.70780 & 8.46050
\end{tabular}




$\begin{array}{cccc}\mathrm{S} & -3.15660 & 4.96980 & 4.94540 \\ \mathrm{O} & -1.92560 & 4.99410 & 4.14780 \\ \mathrm{O} & -4.30990 & 4.21040 & 4.44540 \\ \mathrm{H} & -5.79130 & 6.06760 & 5.20020 \\ \mathrm{H} & -6.68560 & 8.29020 & 5.68460 \\ \mathrm{H} & -5.15840 & 10.20100 & 6.04070 \\ \mathrm{H} & -2.72960 & 9.88430 & 5.89810 \\ \mathrm{H} & -1.27090 & 7.42290 & 7.37640 \\ \mathrm{H} & 1.16030 & 7.47360 & 7.34470 \\ \mathrm{H} & 1.16450 & 6.96760 & 5.67570 \\ \mathrm{H} & 0.56960 & 5.34910 & 8.14840 \\ \mathrm{H} & 1.66330 & 4.97930 & 6.86520 \\ \mathrm{H} & -0.44710 & 4.65530 & 5.43830 \\ \mathrm{H} & -4.89800 & 4.11850 & 6.95150 \\ \mathrm{H} & -2.35480 & 2.99620 & 10.21680 \\ \mathrm{H} & -0.40600 & 3.56400 & 8.90670\end{array}$

REU_651_trans_2

$\begin{array}{lccc}\mathrm{O} & -0.68440 & 8.03220 & 5.01420 \\ \mathrm{C} & -1.50180 & 7.67910 & 5.86520 \\ \mathrm{C} & -2.97490 & 7.76360 & 5.59990 \\ \mathrm{C} & -3.72750 & 6.64810 & 5.15920 \\ \mathrm{C} & -5.10470 & 6.80150 & 4.95010 \\ \mathrm{C} & -5.72770 & 8.03010 & 5.15750 \\ \mathrm{C} & -4.98230 & 9.12650 & 5.57990 \\ \mathrm{C} & -3.61350 & 8.99360 & 5.79980 \\ \mathrm{~N} & -1.19110 & 7.11050 & 7.04220 \\ \mathrm{C} & 0.12430 & 6.58610 & 7.40900 \\ \mathrm{C} & 0.64020 & 5.45070 & 6.48140 \\ \mathrm{~N} & -0.20920 & 4.26860 & 6.29730 \\ \mathrm{C} & -1.38810 & 4.06400 & 7.02990 \\ \mathrm{C} & -2.67320 & 4.33020 & 6.51090 \\ \mathrm{C} & -3.79470 & 4.09200 & 7.31300 \\ \mathrm{C} & -3.67490 & 3.59550 & 8.61810 \\ \mathrm{~N} & -4.87660 & 3.35480 & 9.45350 \\ \mathrm{O} & -4.72450 & 2.91830 & 10.59120 \\ \mathrm{O} & -5.98230 & 3.60050 & 8.97920 \\ \mathrm{C} & -2.39160 & 3.33540 & 9.11450 \\ \mathrm{C} & -1.26490 & 3.56580 & 8.32980 \\ \mathrm{~S} & -3.00650 & 5.00560 & 4.86440 \\ \mathrm{O} & -1.74720 & 5.19770 & 4.13610 \\ \mathrm{O} & -4.05280 & 4.15660 & 4.28100 \\ \mathrm{H} & -5.71030 & 5.96930 & 4.62440 \\ \mathrm{H} & -6.78980 & 8.13180 & 4.99020 \\ \mathrm{H} & -5.46530 & 10.07910 & 5.73990 \\ \mathrm{H} & -3.05340 & 9.85650 & 6.13240 \\ \mathrm{H} & -1.94630 & 6.87080 & 7.66860 \\ \mathrm{H} & 0.04490 & 6.21540 & 8.43130 \\ \mathrm{H} & 0.84500 & 7.40480 & 7.43640 \\ \mathrm{H} & 1.60490 & 5.10170 & 6.84990 \\ \mathrm{H} & 0.84690 & 5.85900 & 5.49210 \\ \mathrm{H} & 0.11810 & 3.53860 & 5.68080\end{array}$




$\begin{array}{cccc}\mathrm{H} & -4.77440 & 4.30630 & 6.91120 \\ \mathrm{H} & -2.25490 & 2.95220 & 10.11500 \\ \mathrm{H} & -0.28850 & 3.35520 & 8.74300\end{array}$

\section{REU_651_trans_3}

$\begin{array}{cccc}\mathrm{O} & -2.13400 & 7.49270 & 8.00320 \\ \mathrm{C} & -2.01540 & 7.41040 & 6.77910 \\ \mathrm{C} & -3.20460 & 7.63630 & 5.88620 \\ \mathrm{C} & -3.71150 & 6.64980 & 5.00050 \\ \mathrm{C} & -4.82610 & 6.95810 & 4.20940 \\ \mathrm{C} & -5.44300 & 8.20360 & 4.29090 \\ \mathrm{C} & -4.95260 & 9.16540 & 5.16720 \\ \mathrm{C} & -3.84250 & 8.88230 & 5.95850 \\ \mathrm{~N} & -0.88240 & 7.05170 & 6.15000 \\ \mathrm{C} & 0.29620 & 6.47880 & 6.80040 \\ \mathrm{C} & 0.75620 & 5.16360 & 6.12570 \\ \mathrm{~N} & -0.19830 & 4.05100 & 6.05520 \\ \mathrm{C} & -1.32860 & 3.99290 & 6.88530 \\ \mathrm{C} & -2.62300 & 4.34620 & 6.44740 \\ \mathrm{C} & -3.69910 & 4.23440 & 7.33400 \\ \mathrm{C} & -3.52300 & 3.78570 & 8.65000 \\ \mathrm{~N} & -4.67460 & 3.68470 & 9.57920 \\ \mathrm{O} & -4.47470 & 3.28390 & 10.72250 \\ \mathrm{O} & -5.78820 & 4.00600 & 9.17350 \\ \mathrm{C} & -2.23190 & 3.43780 & 9.06640 \\ \mathrm{C} & -1.15110 & 3.53660 & 8.19390 \\ \mathrm{~S} & -3.01280 & 4.98410 & 4.79690 \\ \mathrm{O} & -1.78070 & 5.14970 & 4.01790 \\ \mathrm{O} & -4.08410 & 4.12110 & 4.28390 \\ \mathrm{H} & -5.22660 & 6.23170 & 3.51810 \\ \mathrm{H} & -6.30210 & 8.42260 & 3.67410 \\ \mathrm{H} & -5.43110 & 10.13140 & 5.23270 \\ \mathrm{H} & -3.47930 & 9.64760 & 6.63040 \\ \mathrm{H} & -0.88050 & 7.02880 & 5.14020 \\ \mathrm{H} & 0.11770 & 6.29060 & 7.86120 \\ \mathrm{H} & 1.09850 & 7.21550 & 6.75390 \\ \mathrm{H} & 1.64690 & 4.79680 & 6.63630 \\ \mathrm{H} & 1.06920 & 5.38700 & 5.10530 \\ \mathrm{H} & 0.16190 & 3.17930 & 5.69410 \\ \mathrm{H} & -4.68440 & 4.51480 & 6.99050 \\ \mathrm{H} & -2.05340 & 3.08580 & 10.07180 \\ \mathrm{H} & -0.16800 & 3.25680 & 8.54560\end{array}$

\begin{tabular}{lccc}
\multicolumn{4}{l}{ REU_651_trans_4 } \\
O & -0.80250 & 7.63750 & 4.25370 \\
C & -1.38400 & 7.64150 & 5.34040 \\
C & -2.87690 & 7.73600 & 5.39640 \\
C & -3.71020 & 6.61010 & 5.19180 \\
C & -5.10030 & 6.78430 & 5.23780 \\
C & -5.65960 & 8.03910 & 5.46810 \\
C & -4.83570 & 9.14270 & 5.66460 \\
C & -3.45190 & 8.99160 & 5.62920
\end{tabular}




\begin{tabular}{|c|c|c|c|}
\hline $\mathrm{N}$ & -0.78900 & 7.45240 & 6.53200 \\
\hline $\mathrm{C}$ & 0.58130 & 6.95840 & 6.68590 \\
\hline $\mathrm{C}$ & 0.65400 & 5.49320 & 7.17550 \\
\hline $\mathrm{N}$ & -0.30650 & 4.58210 & 6.54070 \\
\hline $\mathrm{C}$ & -1.48690 & 4.25610 & 7.23760 \\
\hline $\mathrm{C}$ & -2.76700 & 4.33200 & 6.64060 \\
\hline $\mathrm{C}$ & -3.89690 & 3.98380 & 7.39150 \\
\hline $\mathrm{C}$ & -3.78050 & 3.54750 & 8.71000 \\
\hline $\mathrm{N}$ & -4.97650 & 3.19030 & 9.47600 \\
\hline $\mathrm{O}$ & -5.52280 & 4.07860 & 10.12060 \\
\hline $\mathrm{O}$ & -5.35700 & 2.02590 & 9.42310 \\
\hline $\mathrm{C}$ & -2.52010 & 3.45330 & 9.29370 \\
\hline $\mathrm{C}$ & -1.38780 & 3.80140 & 8.56280 \\
\hline$S$ & -3.09620 & 4.91280 & 4.95920 \\
\hline $\mathrm{O}$ & -1.84320 & 4.94820 & 4.19700 \\
\hline $\mathrm{O}$ & -4.21370 & 4.10810 & 4.44890 \\
\hline $\mathrm{H}$ & -5.76550 & 5.94420 & 5.10640 \\
\hline $\mathrm{H}$ & -6.73300 & 8.15410 & 5.50020 \\
\hline $\mathrm{H}$ & -5.26890 & 10.11560 & 5.84450 \\
\hline $\mathrm{H}$ & -2.82920 & 9.86240 & 5.78080 \\
\hline $\mathrm{H}$ & -1.34930 & 7.48880 & 7.37150 \\
\hline $\mathrm{H}$ & 1.07900 & 7.60470 & 7.40930 \\
\hline $\mathrm{H}$ & 1.14700 & 7.05200 & 5.75660 \\
\hline $\mathrm{H}$ & 0.52240 & 5.48840 & 8.25710 \\
\hline $\mathrm{H}$ & 1.66330 & 5.11270 & 7.01750 \\
\hline $\mathrm{H}$ & -0.39440 & 4.68950 & 5.54030 \\
\hline $\mathrm{H}$ & -4.88160 & 4.05830 & 6.95280 \\
\hline $\mathrm{H}$ & -2.41590 & 3.10860 & 10.31220 \\
\hline $\mathrm{H}$ & -0.42420 & 3.69940 & 9.04090 \\
\hline \multicolumn{4}{|c|}{ REU_651_cis_1 } \\
\hline $\mathrm{O}$ & 14.60300 & -6.41750 & 3.41870 \\
\hline $\mathrm{C}$ & 14.36310 & -5.87510 & 2.34120 \\
\hline $\mathrm{C}$ & 13.67780 & -4.53510 & 2.31790 \\
\hline $\mathrm{C}$ & 12.28560 & -4.36160 & 2.51450 \\
\hline $\mathrm{C}$ & 11.76050 & -3.06200 & 2.51670 \\
\hline $\mathrm{C}$ & 12.58080 & -1.95160 & 2.33870 \\
\hline $\mathrm{C}$ & 13.94670 & -2.12340 & 2.14770 \\
\hline $\mathrm{C}$ & 14.48920 & -3.40530 & 2.13890 \\
\hline $\mathrm{N}$ & 14.78740 & -6.39900 & 1.17280 \\
\hline $\mathrm{C}$ & 14.29800 & -6.07310 & -0.17220 \\
\hline $\mathrm{C}$ & 13.28070 & -7.10760 & -0.69720 \\
\hline $\mathrm{N}$ & 12.11640 & -7.26820 & 0.16990 \\
\hline $\mathrm{C}$ & 10.96840 & -6.49200 & -0.05450 \\
\hline $\mathrm{C}$ & 10.35580 & -5.78310 & 0.99760 \\
\hline $\mathrm{C}$ & 9.19540 & -5.04200 & 0.74160 \\
\hline $\mathrm{C}$ & 8.62000 & -4.99590 & -0.53560 \\
\hline $\mathrm{N}$ & 7.39090 & -4.20480 & -0.78880 \\
\hline $\mathrm{O}$ & 6.88360 & -3.58950 & 0.14490 \\
\hline $\mathrm{O}$ & 6.92280 & -4.19220 & -1.92400 \\
\hline $\mathrm{C}$ & 9.23750 & -5.71150 & -1.56820 \\
\hline $\mathrm{C}$ & 10.39440 & -6.44860 & -1.33100 \\
\hline
\end{tabular}




$\begin{array}{cccc}\mathrm{S} & 11.06110 & -5.69140 & 2.66300 \\ \mathrm{O} & 10.00840 & -5.20380 & 3.56350 \\ \mathrm{O} & 11.72300 & -6.96560 & 2.96400 \\ \mathrm{H} & 10.69970 & -2.90210 & 2.64230 \\ \mathrm{H} & 12.15550 & -0.95890 & 2.34210 \\ \mathrm{H} & 14.58600 & -1.26430 & 2.00810 \\ \mathrm{H} & 15.55560 & -3.51240 & 1.99590 \\ \mathrm{H} & 15.33030 & -7.24930 & 1.22250 \\ \mathrm{H} & 15.16250 & -6.05410 & -0.83600 \\ \mathrm{H} & 13.86150 & -5.07430 & -0.21690 \\ \mathrm{H} & 12.98350 & -6.84000 & -1.71090 \\ \mathrm{H} & 13.76190 & -8.08160 & -0.79020 \\ \mathrm{H} & 12.34550 & -7.42700 & 1.14070 \\ \mathrm{H} & 8.74090 & -4.48050 & 1.54400 \\ \mathrm{H} & 8.82460 & -5.70560 & -2.56630 \\ \mathrm{H} & 10.82990 & -7.00060 & -2.15120\end{array}$

REU_651_cis_2

\begin{tabular}{lccc}
$\mathrm{O}$ & 14.85840 & -6.23970 & 3.39740 \\
$\mathrm{C}$ & 14.42100 & -5.77210 & 2.34740 \\
$\mathrm{C}$ & 13.69240 & -4.45740 & 2.36510 \\
$\mathrm{C}$ & 12.28870 & -4.34800 & 2.51060 \\
$\mathrm{C}$ & 11.71030 & -3.07170 & 2.53670 \\
$\mathrm{C}$ & 12.49120 & -1.92410 & 2.42910 \\
$\mathrm{C}$ & 13.87010 & -2.03470 & 2.28960 \\
$\mathrm{C}$ & 14.46510 & -3.29300 & 2.25910 \\
$\mathrm{~N}$ & 14.62740 & -6.37570 & 1.16090 \\
$\mathrm{C}$ & 14.03300 & -6.04110 & -0.13640 \\
$\mathrm{C}$ & 13.17610 & -7.19310 & -0.69500 \\
$\mathrm{~N}$ & 11.99340 & -7.52160 & 0.10270 \\
$\mathrm{C}$ & 10.89960 & -6.65890 & -0.08000 \\
$\mathrm{C}$ & 10.40940 & -5.82740 & 0.94840 \\
$\mathrm{C}$ & 9.31090 & -4.99760 & 0.69000 \\
$\mathrm{C}$ & 8.66980 & -4.98840 & -0.55600 \\
$\mathrm{~N}$ & 7.50840 & -4.10080 & -0.80830 \\
$\mathrm{O}$ & 7.12030 & -3.36700 & 0.09660 \\
$\mathrm{O}$ & 6.97510 & -4.12970 & -1.91400 \\
$\mathrm{C}$ & 9.15140 & -5.84110 & -1.55560 \\
$\mathrm{C}$ & 10.24620 & -6.66680 & -1.31820 \\
$\mathrm{~S}$ & 11.12830 & -5.73830 & 2.60800 \\
$\mathrm{O}$ & 10.05430 & -5.32580 & 3.52090 \\
$\mathrm{O}$ & 11.84990 & -6.98610 & 2.88110 \\
$\mathrm{H}$ & 10.64060 & -2.95910 & 2.63290 \\
$\mathrm{H}$ & 12.02600 & -0.94960 & 2.45030 \\
$\mathrm{H}$ & 14.47840 & -1.14630 & 2.20570 \\
$\mathrm{H}$ & 15.53940 & -3.35430 & 2.15310 \\
$\mathrm{H}$ & 15.17130 & -7.22670 & 1.16660 \\
$\mathrm{H}$ & 14.84690 & -5.82580 & -0.82900 \\
$\mathrm{H}$ & 13.43100 & -5.13240 & -0.09730 \\
$\mathrm{H}$ & 12.87180 & -6.93300 & -1.70960 \\
$\mathrm{H}$ & 13.78480 & -8.09240 & -0.79380 \\
$\mathrm{H}$ & 11.68620 & -8.43910 & -0.18660 \\
& & & \\
\hline
\end{tabular}




$\begin{array}{cccc}\mathrm{H} & 8.94900 & -4.34750 & 1.47250 \\ \mathrm{H} & 8.68170 & -5.87170 & -2.52780 \\ \mathrm{H} & 10.58770 & -7.31530 & -2.11270\end{array}$

REU_651_cis_3

$\begin{array}{cccc}\mathrm{O} & 13.97750 & -6.85010 & 3.65360 \\ \mathrm{C} & 14.12300 & -6.29250 & 2.56690 \\ \mathrm{C} & 13.84780 & -4.81320 & 2.45430 \\ \mathrm{C} & 12.54260 & -4.25000 & 2.49450 \\ \mathrm{C} & 12.40470 & -2.85740 & 2.42830 \\ \mathrm{C} & 13.51740 & -2.02670 & 2.33300 \\ \mathrm{C} & 14.79320 & -2.57760 & 2.29480 \\ \mathrm{C} & 14.95520 & -3.95880 & 2.35650 \\ \mathrm{~N} & 14.59000 & -6.96710 & 1.49170 \\ \mathrm{C} & 14.62940 & -6.56010 & 0.07920 \\ \mathrm{C} & 13.24410 & -6.53010 & -0.60270 \\ \mathrm{~N} & 12.49680 & -5.35460 & -0.19940 \\ \mathrm{C} & 11.10670 & -5.37030 & -0.32140 \\ \mathrm{C} & 10.30340 & -5.21320 & 0.82310 \\ \mathrm{C} & 8.91250 & -5.17230 & 0.67690 \\ \mathrm{C} & 8.30440 & -5.28630 & -0.58230 \\ \mathrm{~N} & 6.82810 & -5.24050 & -0.72020 \\ \mathrm{O} & 6.14650 & -5.09290 & 0.29040 \\ \mathrm{O} & 6.33810 & -5.35130 & -1.84070 \\ \mathrm{C} & 9.12460 & -5.45420 & -1.70660 \\ \mathrm{C} & 10.51080 & -5.49780 & -1.57850 \\ \mathrm{~S} & 10.97740 & -5.18590 & 2.50950 \\ \mathrm{O} & 10.03920 & -4.40880 & 3.32940 \\ \mathrm{O} & 11.23690 & -6.58840 & 2.84980 \\ \mathrm{H} & 11.42330 & -2.40640 & 2.43360 \\ \mathrm{H} & 13.38940 & -0.95560 & 2.28090 \\ \mathrm{H} & 15.65770 & -1.93470 & 2.21950 \\ \mathrm{H} & 15.95840 & -4.36140 & 2.33350 \\ \mathrm{H} & 14.81810 & -7.93960 & 1.64120 \\ \mathrm{H} & 15.26830 & -7.26630 & -0.45130 \\ \mathrm{H} & 15.13090 & -5.59510 & -0.01400 \\ \mathrm{H} & 13.34480 & -6.49840 & -1.68800 \\ \mathrm{H} & 12.69590 & -7.44250 & -0.36090 \\ \mathrm{H} & 12.78380 & -5.03510 & 0.71490 \\ \mathrm{H} & 8.29680 & -5.06970 & 1.55830 \\ \mathrm{H} & 8.69440 & -5.54860 & -2.69290 \\ \mathrm{H} & 11.11880 & -5.61410 & -2.46440 \\ & & & \\ & & & \end{array}$

REU_651_cis_4

$\begin{array}{llll}\mathrm{O} & 12.11920 & -2.66350 & 0.69900 \\ \mathrm{C} & 12.81630 & -3.67640 & 0.68280 \\ \mathrm{C} & 13.42460 & -4.18440 & 1.95730 \\ \mathrm{C} & 12.66350 & -4.84790 & 2.94800 \\ \mathrm{C} & 13.30800 & -5.29210 & 4.11000 \\ \mathrm{C} & 14.67450 & -5.09440 & 4.29370 \\ \mathrm{C} & 15.41980 & -4.44170 & 3.31730 \\ \mathrm{C} & 14.79770 & -3.98730 & 2.15740\end{array}$




\begin{tabular}{|c|c|c|c|}
\hline $\mathrm{N}$ & 13.03580 & -4.37180 & -0.45410 \\
\hline $\mathrm{C}$ & 13.73020 & -5.65490 & -0.67590 \\
\hline $\mathrm{C}$ & 13.59800 & -6.79680 & 0.38040 \\
\hline $\mathrm{N}$ & 12.26650 & -7.18220 & 0.85940 \\
\hline $\mathrm{C}$ & 11.09990 & -6.56610 & 0.38910 \\
\hline $\mathrm{C}$ & 10.38370 & -5.63810 & 1.16730 \\
\hline $\mathrm{C}$ & 9.23230 & -5.04650 & 0.63370 \\
\hline $\mathrm{C}$ & 8.78260 & -5.34900 & -0.65950 \\
\hline $\mathrm{N}$ & 7.56420 & -4.70730 & -1.21090 \\
\hline $\mathrm{O}$ & 7.20580 & -5.00420 & -2.34720 \\
\hline $\mathrm{O}$ & 6.95580 & -3.90090 & -0.51270 \\
\hline $\mathrm{C}$ & 9.51320 & -6.27150 & -1.41890 \\
\hline $\mathrm{C}$ & 10.65660 & -6.87270 & -0.90040 \\
\hline S & 10.87650 & -5.15730 & 2.84340 \\
\hline $\mathrm{O}$ & 10.23940 & -3.86790 & 3.13690 \\
\hline $\mathrm{O}$ & 10.58650 & -6.32530 & 3.68390 \\
\hline $\mathrm{H}$ & 12.75170 & -5.79340 & 4.88800 \\
\hline $\mathrm{H}$ & 15.15450 & -5.44470 & 5.19550 \\
\hline $\mathrm{H}$ & 16.47910 & -4.28650 & 3.45910 \\
\hline $\mathrm{H}$ & 15.39510 & -3.48300 & 1.41070 \\
\hline $\mathrm{H}$ & 12.59720 & -4.00290 & -1.28580 \\
\hline $\mathrm{H}$ & 14.78770 & -5.43180 & -0.82080 \\
\hline $\mathrm{H}$ & 13.36960 & -6.02640 & -1.63530 \\
\hline $\mathrm{H}$ & 14.06960 & -7.69520 & -0.01810 \\
\hline $\mathrm{H}$ & 14.19850 & -6.56000 & 1.25660 \\
\hline $\mathrm{H}$ & 12.21060 & -7.81430 & 1.64510 \\
\hline $\mathrm{H}$ & 8.67850 & -4.33950 & 1.23320 \\
\hline $\mathrm{H}$ & 9.20150 & -6.53140 & -2.41990 \\
\hline $\mathrm{H}$ & 11.20070 & -7.57910 & -1.51120 \\
\hline \multicolumn{4}{|c|}{ REU_651_cis_5 } \\
\hline $\mathrm{O}$ & 12.16410 & -3.28370 & -0.10100 \\
\hline $\mathrm{C}$ & 12.92390 & -4.12520 & 0.37560 \\
\hline $\mathrm{C}$ & 13.32930 & -4.03090 & 1.81880 \\
\hline $\mathrm{C}$ & 12.54860 & -4.53140 & 2.89180 \\
\hline $\mathrm{C}$ & 13.00460 & -4.33750 & 4.20200 \\
\hline $\mathrm{C}$ & 14.19910 & -3.67200 & 4.46250 \\
\hline $\mathrm{C}$ & 14.96520 & -3.18800 & 3.40880 \\
\hline $\mathrm{C}$ & 14.53110 & -3.36520 & 2.09820 \\
\hline $\mathrm{N}$ & 13.45660 & -5.11220 & -0.37530 \\
\hline $\mathrm{C}$ & 14.15450 & -6.31810 & 0.09060 \\
\hline $\mathrm{C}$ & 13.35380 & -7.21410 & 1.06860 \\
\hline $\mathrm{N}$ & 12.09550 & -7.73110 & 0.53660 \\
\hline $\mathrm{C}$ & 10.99360 & -6.89040 & 0.27450 \\
\hline $\mathrm{C}$ & 10.48470 & -5.84420 & 1.08840 \\
\hline $\mathrm{C}$ & 9.44150 & -5.04760 & 0.59180 \\
\hline $\mathrm{C}$ & 8.86540 & -5.27240 & -0.66420 \\
\hline $\mathrm{N}$ & 7.76530 & -4.41210 & -1.16480 \\
\hline $\mathrm{O}$ & 7.28750 & -4.65200 & -2.27030 \\
\hline $\mathrm{O}$ & 7.37050 & -3.48900 & -0.45780 \\
\hline $\mathrm{C}$ & 9.34820 & -6.33580 & -1.43080 \\
\hline $\mathrm{C}$ & 10.39050 & -7.12900 & -0.96470 \\
\hline
\end{tabular}




$\begin{array}{cccc}\mathrm{S} & 11.00780 & -5.49470 & 2.79100 \\ \mathrm{O} & 9.98550 & -4.62990 & 3.39340 \\ \mathrm{O} & 11.28390 & -6.79390 & 3.41630 \\ \mathrm{H} & 12.43070 & -4.71670 & 5.03520 \\ \mathrm{H} & 14.53210 & -3.53640 & 5.48090 \\ \mathrm{H} & 15.89310 & -2.67200 & 3.60650 \\ \mathrm{H} & 15.13980 & -2.97060 & 1.29630 \\ \mathrm{H} & 13.21130 & -5.12360 & -1.35490 \\ \mathrm{H} & 15.11250 & -6.04040 & 0.53230 \\ \mathrm{H} & 14.39340 & -6.89570 & -0.80290 \\ \mathrm{H} & 13.95780 & -8.08400 & 1.32660 \\ \mathrm{H} & 13.22700 & -6.70490 & 2.00950 \\ \mathrm{H} & 12.23210 & -8.43250 & -0.17710 \\ \mathrm{H} & 9.05830 & -4.23880 & 1.19490 \\ \mathrm{H} & 8.92840 & -6.55130 & -2.40240 \\ \mathrm{H} & 10.74880 & -7.92500 & -1.60310\end{array}$




\section{References}

1. Sheldrick, G.M. Crystal structure refinement with SHELXL, Acta Crystallographica Section C, 2015, 71, 3-8

2. Dolomanov, O.V.; Bourhis, L. J.; Gildea, R. J.; Howard, J. A. K.; Puschmann, H. OLEX2: a complete structure solution, refinement and analysis program. J. Appl. Cryst. 2009, 42, 339-341.

3. CrysAlisPro, Agilent Technologies Ltd., Version 1.171.136.120 (release 127-106-2012).

4. Zhang, K.; Teklebrhan, R. B.; Schreckenbach, G.; Wetmore, S.; Schweizer, F., Intramolecular Hydrogen Bond-Controlled Prolyl Amide Isomerization in Glucosyl 3'(S)-Hydroxy-5'hydroxymethylproline Hybrids: Influence of a C-5'-Hydroxymethyl Substituent on the Thermodynamics and Kinetics of Prolyl Amide Cis/Trans Isomerization. The Journal of Organic Chemistry 2009, 74 (10), 3735-3743.

5. Kessler, H., Conformation and Biological Activity of Cyclic Peptides. Angewandte Chemie International Edition in English 1982, 21 (7), 512-523.

6. Stevens, E. S.; Sugawara, N.; Bonora, G. M.; Toniolo, C., Conformational analysis of linear peptides. 3. Temperature dependence of $\mathrm{NH}$ chemical shifts in chloroform. Journal of the American Chemical Society 1980, 102 (23), 7048-7050.

7. Tasi, G.; Mizukami, F.; Pálinkó, I.; Csontos, J.; Győrffy, W.; Nair, P.; Maeda, K.; Toba, M.; Niwa, S.-i.; Kiyozumi, Y.; Kiricsi, I., Enumeration of the Conformers of Unbranched Aliphatic Alkanes. The Journal of Physical Chemistry A 1998, 102 (39), 7698-7703. 